Die numerische Kompetenz von Vorschulkindern - Theoretische Modelle und empirische Befunde

\author{
Von dem Fachbereich Erziehungswissenschaften \\ der Universität Hannover \\ zur Erlangung des Grades eines
}

DOKTORS DER PHILOSOPIE

Dr. phil.

genehmigte Dissertation

von

Dipl. Math. Franco Caluori

geboren ab 8. Juni 1951. Bürger von Bonaduz (CH).

2004 
Referent:

Prof. Dr. K. Hasemann, Universität Hannover

Korreferenten: $\quad$ Prof. Dr. F. Käpnick, Technische Universität Braunschweig Prof. Dr. H. Wippermann, Universität Hannover

Tag der Promotion: 10. Dezember 2003 


\title{
Die numerische Kompetenz von Vorschulkindern - Theoretische Modelle und empirische Befunde
}

\section{Zusammenfassung}

Der Beginn des Bildungsgangs verläuft in Deutschland und in der Schweiz bedingt durch die Trennung von Kindergarten und Grundschule gebrochen. In Zuge der Diskussion der Ergebnisse der TIMMS und PISA Studien stellt sich die Frage, ob diese Trennung der Eingangsstufe in Kindergarten und Grundschule gerechtfertigt ist oder ob eine Neuorganisation dieser Eingangsstufe etwa im Sinne des holländischen Systems mit jahrgangsgemischten Niveaustufen ins Auge zu fassen ist?

Nach einer breiten theoretischen Untersuchung zur Zahlbegriffsentwicklung und zur Zählkompetenz werden in dieser Arbeit die mathematischen Kompetenzen von Vorschulkindern erhoben und insbesondere die individuellen Unterschiede bei der Lösung von numerischen und nichtnumerischen Aufgaben untersucht. Zu dieser Kompetenzerhebung wurde der „Osnabrücker Test zur Zahlbegriffentwicklung (OTZ)" verwendet. Im Folgenden werden die Ergebnisse dieser Schweizerischen Kompetenzerhebung verglichen mit Ergebnissen aus Deutschland und Holland. Weiter wird der Frage nach dem Einfluss der pränumerischen Komponenten im Sinne Piaget's (Vergleichen, Klassifizieren, Zuordnen und Reihenfolgenbildung) auf die Zählkompetenz und auf einfache numerische Aufgaben nachgegangen. Es ergeben sich kognitive Voraussetzungen, die für einen erfolgreichen mathematischen Anfangsunterricht erforderlich sind. Zum Schluss werden praktische Folgerungen abgeleitet für eine optimale Förderung der Kinder im Kindergarten sowie eine Empfehlung zur Gestaltung des Übergangs Kindergarten/Grundschule als jahrgangsübergreifende Basisstufe abgegeben.

\section{Stichworte}

Zählkompetenz, OTZ, Basisstufe

\begin{abstract}
The beginning of the course of instruction is not taking its normal course in Germany and Switzerland conditional upon separation from Kindergarten and elementary school. After the outcomes of the TIMMS and PISA studies it is questionable whether this separation is justified. Therefore, a new organisation is to think over especially on this level just as the Dutch System witch its mixed stage of developments.

After a comprehensive and theoretical examination for numerical competence and conceptive competency of numbers in this depiction the mathematical competence of Kindergarten children's and particular the individuell difference of the solution of numerical and non-numerical tasks should be analysed. The OTZ was expended on this competence elevation of numerical deployment. The results of this Swiss competence elevation are subsequently compared to the results of Germany and Holland. Furthermore, one cannot evade the Point according to the influence of pre-numerical competence in a sense of Piaget (compare, classify, one-to-one-assignment and sequence) to the numerical competence and for simple numerical tasks. Hence, is follows cognitive conditions which are request for a successful mathematical start in the elementary-school. Finally, practical conclusions are to be derived to obtain an optimal promotion of the nursery-school as well as a recommendation to construct the beginning of the course of instruction.
\end{abstract}

\section{Keywords}

Competence of counting, OTZ, nursery-school 


\section{Inhaltsverzeichnis}

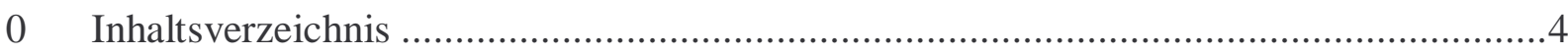

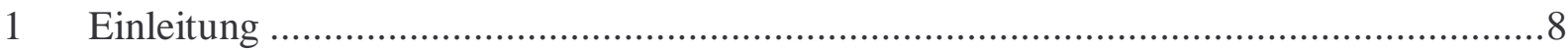

Fragestellungen und Aufbau der Arbeit ................................................ 11

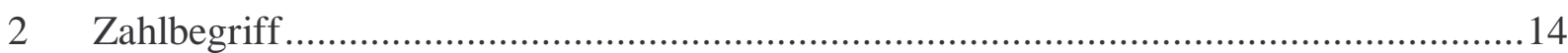

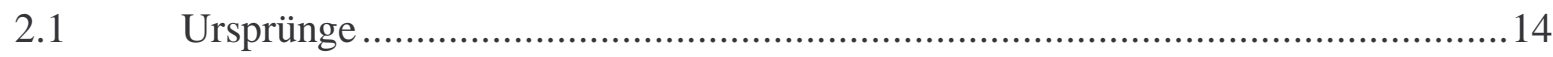

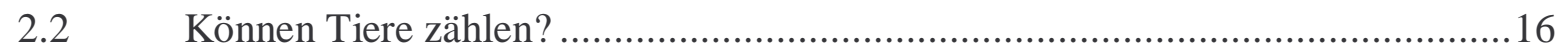

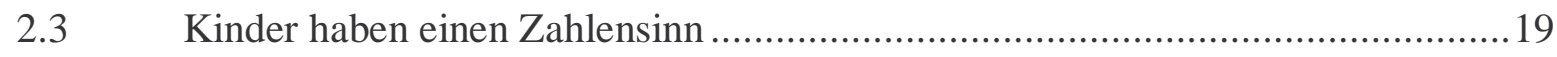

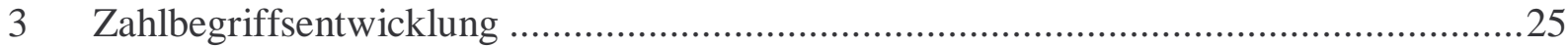

3.1 Geschichtliches und Fachliches zur Entwicklung des Zahlbegriffs ...................25

Kognitive Strukturen nach Piaget .........................................................29

Entwicklung und Lernen nach Piaget ........................................................ 32

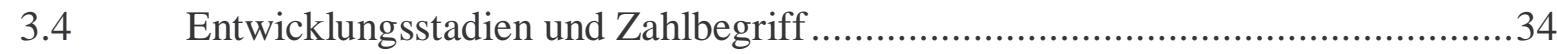

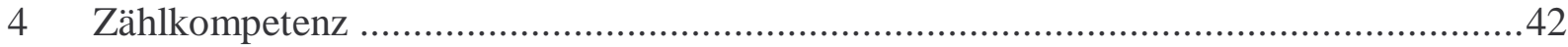

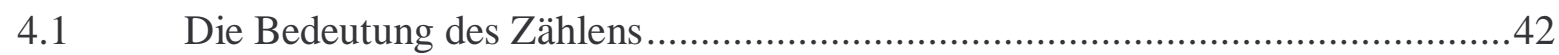

4.2 Die Entwicklung des Zählens .............................................................44

4.2.1 Die Prinzipien-Vorher-Theorie .............................................................. 44

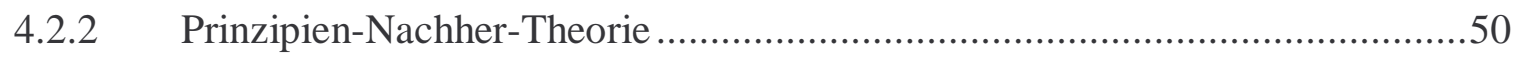

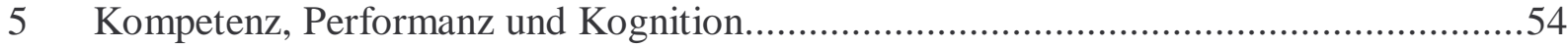

Kompetenz und Performanz.................................................................. 54

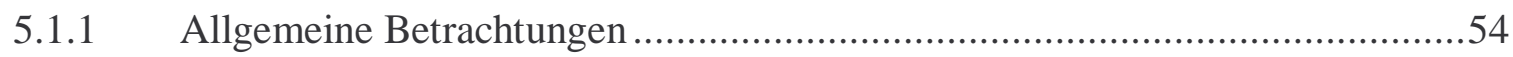

5.1.2 Theoretische Modelle und Hintergründe .................................................56

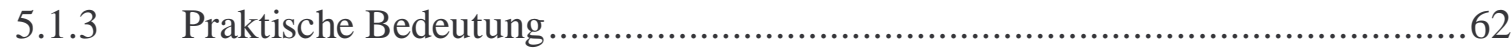

5.1.4 Kompetenz und der Erwerb von numerischen Grundlagen ..........................63

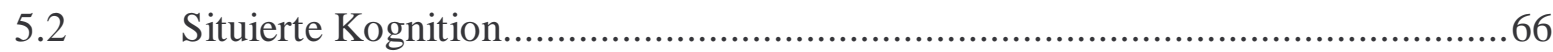


5.3 Mentale Modelle. 70

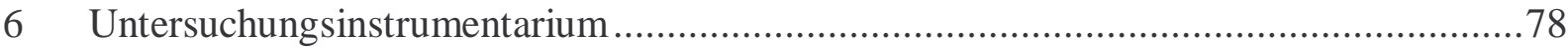

6.1 Osnabrücker Test zur Zahlbegriffsentwicklung ..........................................78

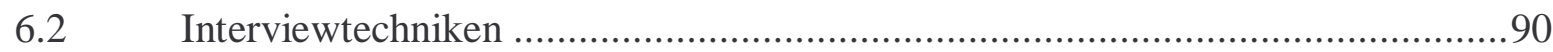

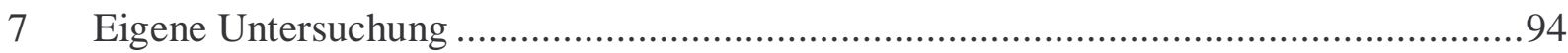

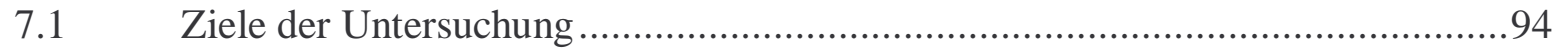

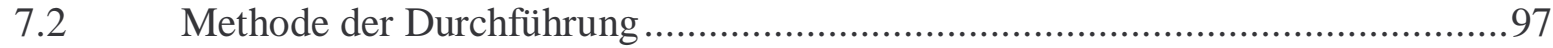

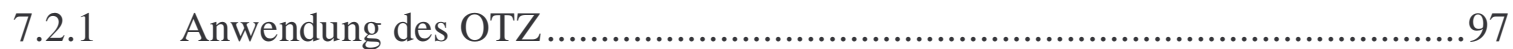

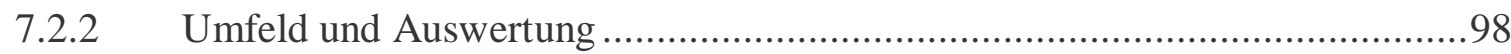

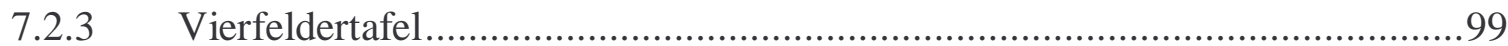

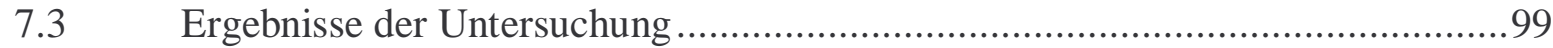

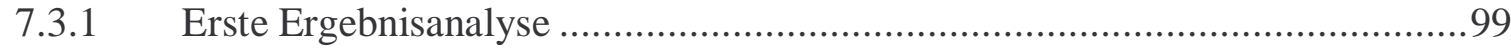

7.3.2 Die Ergebnisse der acht Komponenten ...................................................... 101

7.3.3 Die Ergebnisse der einzelnen Aufgaben des OTZ......................................103

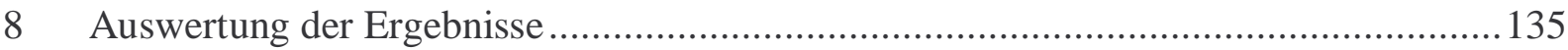

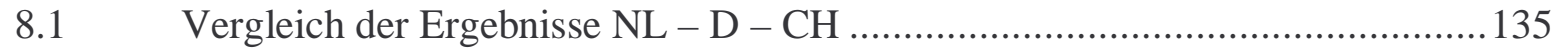

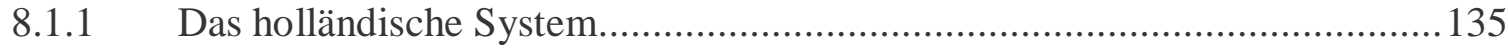

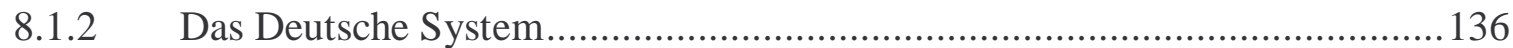

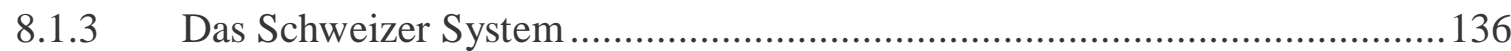

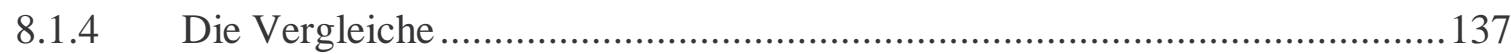

8.2 Die Piaget'schen Komponenten in Wechselwirkung zur Zählkompetenz..........142

8.2.1 Die Vergleichskonzepte in Wechselwirkung mit Zählkompetenz .................143

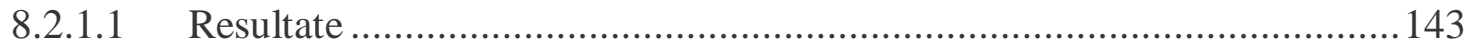

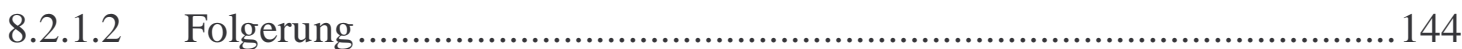

8.2.2 Die Klassifizierung in Wechselwirkung mit Zählkompetenz ....................... 145

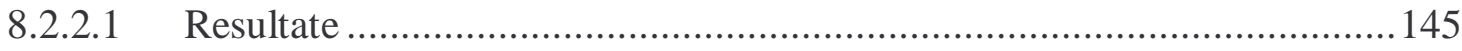




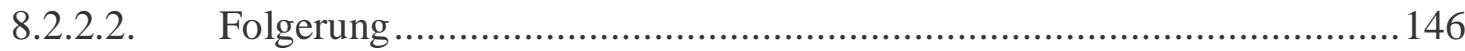

8.2.3 Die Zuordnungskomponente in Wechselwirkung mit Zählkompetenz .......... 146

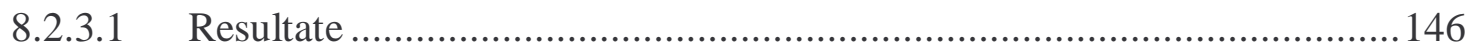

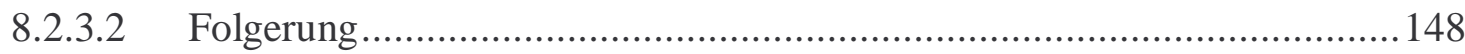

8.2.4 Die Reihenfolgekomponente in Wechselwirkung mit Zählkompetenz ........... 148

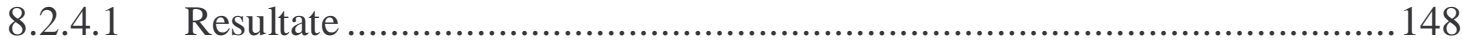

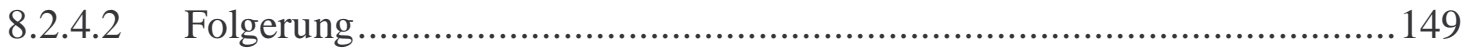

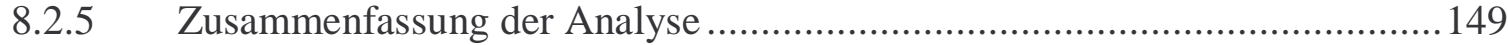

8.2.5.1 Die Abhängigkeit der Erfolge in den vier Komponenten vom Alter......... 150

8.2.5.2 Die Abhängigkeit der Erfolge von der allgemeinen Leistungsfähigkeit.... 151

8.3 Pränumerische und numerische Kompetenzen im Vergleich ..........................155

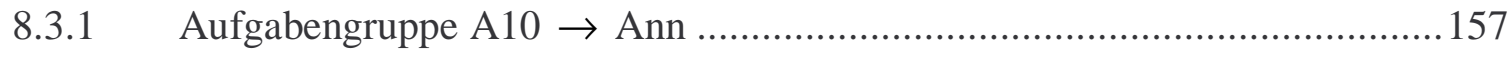

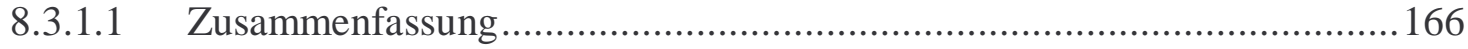

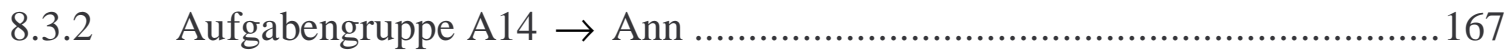

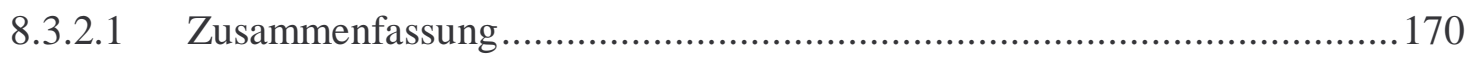

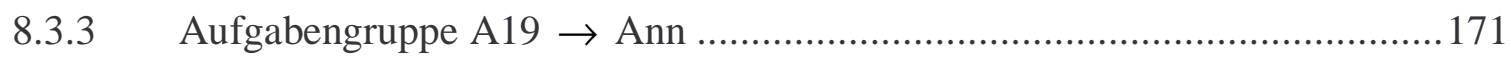

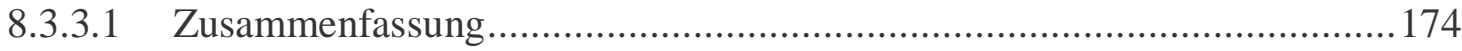

8.3.4 Aufgabengruppe A21 $\rightarrow$ Ann ….................................................... 175

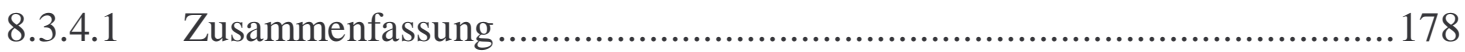

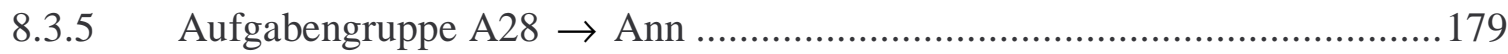

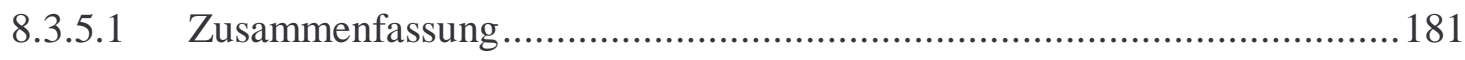

8.3.6 Aufgabengruppe A23 $\rightarrow$ Ann …................................................... 181

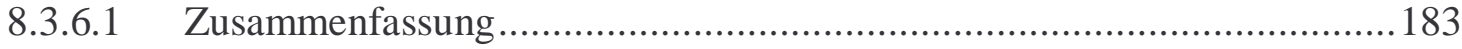

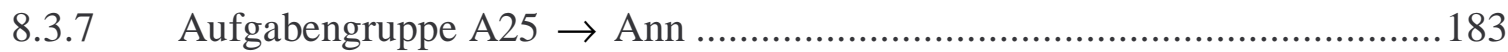

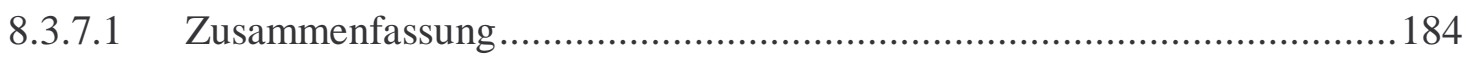

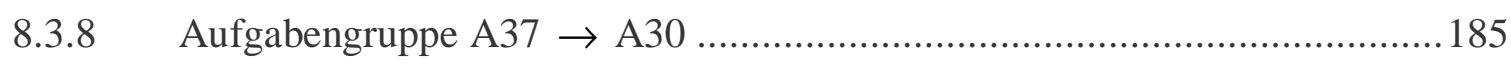

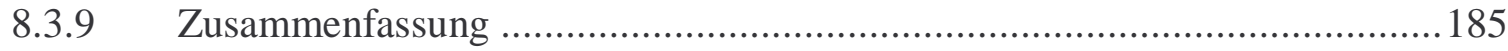

$9 \quad$ Kompetenzentwicklung und -unterschiede einzelner Kinder....................................190

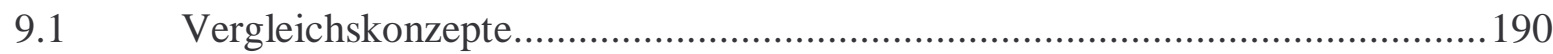




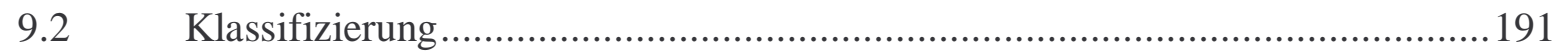

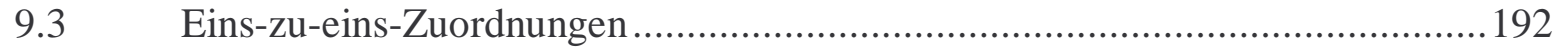

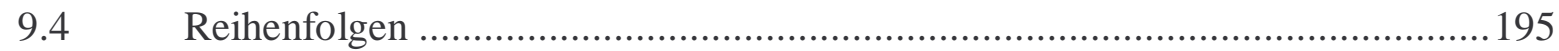

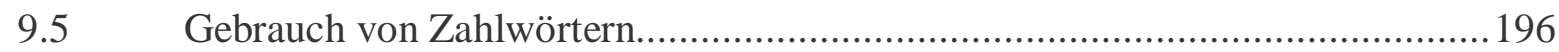

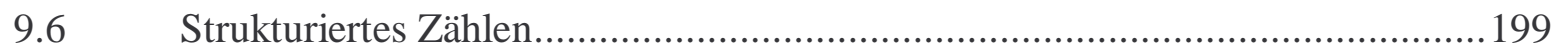

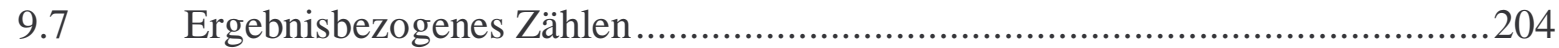

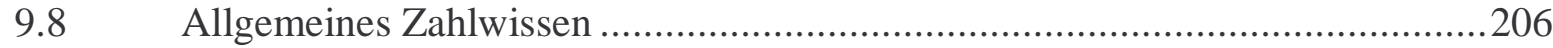

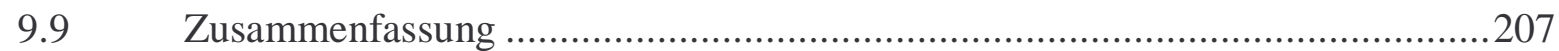

10 Vorschulische Förderung und Basisstufe .............................................................210

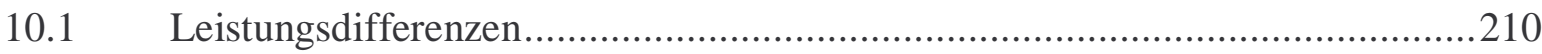

$10.2 \quad$ Mathematische Förderung in der Vorschulzeit ..........................................213

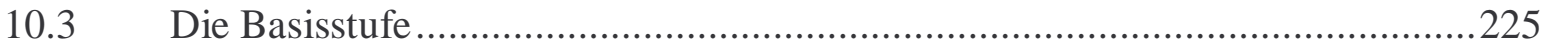

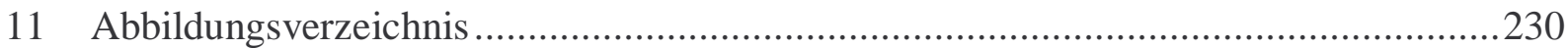

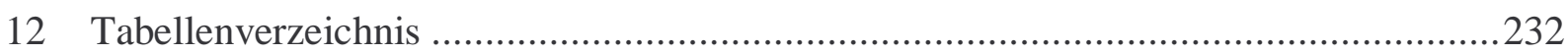

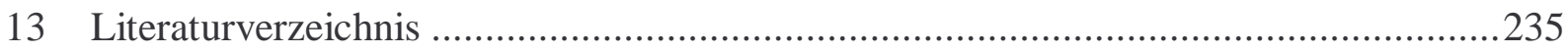

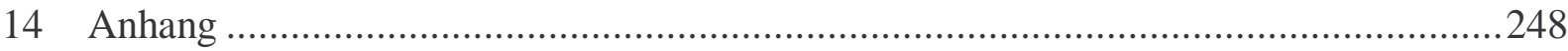

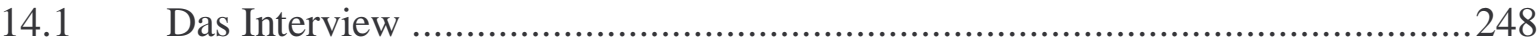

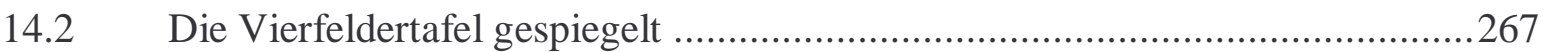

14.3 Vierfeldertafeln Alter und allgemeine Leistungsfähigkeit..............................268

14.4 Ergebnisstabellen des „Item-Muster“ Verfahrens ........................................2270

$14.5 \quad$ Alle Verknüpfungen des „Item-Muster“-Verfahrens ......................................2272 


\section{$1 \quad$ Einleitung}

Es wird allgemein anerkannt, dass Rechnen und der Umgang mit Ziffern und Zahlen genauso wie Lesen und Schreiben zu den fundamentalen Kulturtechniken gezählt wird. Die Frage, wie Kinder Zugang und Kenntnis über eine zentrale Kulturtechnik wie Mathematik erlangen, ist in dieser Arbeit von besonderem Interesse. Mathematik entstand aus praktischen Problemen des Zählens, Messens, Rechnens und geometrischen Zeichnens, um sich dann im Laufe von vielen Jahrhunderten weiterzuentwickeln zu einer hoch abstrakten Wissenschaft im Sinne von Wittenberg (1968), der die Mathematik als Experiment des reinen Denkens betrachtet. Die Schulmathematik liegt nun irgendwo zwischen diesen zwei Extrempositionen der praktischen Anwendung und des reinen Denkens. Um Zugang zu dieser Wissenschaft zu erlangen, sind mannigfaltige Kenntnisse und Erkenntnisse notwendig; die Mathematik erfordert aber zusätzlich das Erlernen einer formalen Sprache, in der die mathematischen Sachverhalte ausgedrückt werden können. Erste Kenntnisse und Einsichten, aber auch Ansätze zu dieser formalen Sprache erwerben die Kinder bereits in ihren ersten Lebensjahren, wobei erhebliche individuelle Unterschiede feststellbar sind. Im Rahmen der mathematischen Grundausbildung sollen alle Kinder Zugang zu dieser formalen Sprache finden und fähig sein, dieses eigenständig und kritisch zu nutzen. Um dieses Ziel zu erreichen, ist ein langer und oft mühsamer Weg zurückzulegen, wobei uns in besonderem Maß der Beginn dieses Weges interessiert. Konkret wollen wir die numerischen Kompetenzen und mathematischen Lösungsstrategien von Kindern im Vorschulalter genauer untersuchen. Die mathematische Kompetenz von Schulanfängerinnen und Schulanfängern ist in den letzten Jahren wiederholt untersucht worden (Spiegel 1992; Selter 1995; Van den Heuvel-Panhuizen 1995; Hengartner/Röthlisberger 1994 u.a.). Diese Untersuchungen zeigen, dass es mit den mathematischen Kompetenzen der Kinder weit besser bestellt ist als gemeinhin angenommen wurde. Aus allen durchgeführten Studien geht zudem hervor, dass die Kinder auch große individuelle Unterschiede in der mathematischen Kompetenz aufweisen (Fuson 1988; Grassmann 1995). Diese zwei Erkenntnisse haben in den letzten Jahren ihren Niederschlag gefunden nicht nur in der Konzeption von Unterrichtsmaterial und Lehrmitteln, sondern auch, und das scheint mir weitaus wichtiger und nachhaltiger, in der Mathematikdidaktik. 
Es stellt sich die Frage, wo und wann sich die Kinder diese auf den ersten Blick erstaunlichen Kompetenzen angeeignet haben. Sicher ist hier das Elternhaus zu nennen. Dies ist wohl in den meisten Fällen der wichtigste Ort für die geistige Entwicklung des Kindes. Viele weitere Einflüsse und Anregungen erfährt der junge Mensch aber auch durch Gleichaltrige auf dem Spielplatz oder auf der Strasse. Auch im Umgang mit Spielsachen und Gegenständen macht das junge Kind selbständig vielfältige mathematische Grunderfahrungen. Es vergleicht, sortiert, beobachtet Abläufe, Folgen und Muster, ordnet und zählt. Durch diese Erfahrungen und durch die (oft intensive) Kommunikation mit Eltern, Geschwistern und Spielkameraden entsteht im Laufe der Jahre so etwas wie eine „Alltagsmathematik"; und es entwickelt sich ein Interesse an mathematischen Zusammenhängen. Dabei bilden sich Vorstellungen und oft entsteht eine ungeordnete und zufällige Ansammlung von Begriffen und Ahnungen sowie Erklärungsschemata, die für die mathematische Entwicklung wesentlich sind. Hier wird Neugierde geweckt. Oft beobachtet man Kinder, die eine große Freude am Experimentieren mit ihren noch unfertigen mathematischen Hilfsmitteln und Werkzeugen an den Tag legen.

Ein weiterer Ort, an dem die Kinder mit Mathematik im weitesten Sinn in Berührung kommen, ist der Kindergarten. Viele Kindergärtnerinnen haben in der Praxis seit vielen Jahren mit ihren Kindern bewusst oder unbewusst, direkt oder indirekt Mathematik auf vielfältiger Art und Weise praktiziert. Mathematik ist zum Beispiel nachweisbar in vielen Märchen und Geschichten mit Zahlangaben: „Die vierzig Räuber“; „Die sieben Zwerge“; „Die sieben Raben“ und viele andere. Oder in Abzählverse wie „Zehn kleine Negerlein“ und ebenso bei „Das isch dä Tuuma, dä schüttlet Pfluuma, das isch dä ...“ oder beim „Joggeli“, der Birnen schüttelt, als Ablauf, als Reihenfolge. Die Kinder sollen sich der Größe nach einordnen. Die Geburtstage werden einer nach dem andern im Verlaufe des Jahres gefeiert. Die Mathematik ist dabei beim Rollenspiel: „Du wärst der Vater und du wärst die Mutter und du die Lehrerin und du das Kind und du..." als Zuordnung. Wenn die Zuordnung nicht aufgeht, das heißt, wenn es mehr Kinder als zu vergebende Rollen gibt, so bleiben ein paar Zuschauer übrig. Gibt es aber mehr Rollen als Kinder, so werden auch Doppelrollen zu vergeben sein. Vielleicht sind einmal genau gleich viele Kinder da wie Rollen, dann geht es genau auf. Das führt zu Begriffen wie gleichviel, mehr, weniger, eines fehlt. Dies hängt mit der „Eins-zu-eins-Zuordnung“ zusammen, die zusammen mit der erwähnten Reihenfolgenbildung zur Entwicklung des Zahlbegriffs beiträgt. Mathematik ist dabei beim Aufräumen: „Je weniger Kinder beim Aufräumen helfen, desto mehr Zeit brauchen wir dafür.“ Oder positiver: „Je mehr Kin- 
der beim Aufräumen helfen, desto weniger Zeit brauchen wir dafür." Viele weitere Beispiele ließen sich aufzählen (vgl. Schmassmann 1986). Dieser Umgang mit mathematischen Sachverhalten ist für viele Kinder etwas ganz Alltägliches. Die Kinder entwickeln aber auch von sich aus Freude und Lust am mathematischen Tun, das von vielen Kindergärtnerinnen ganz selbstverständlich unterstützt und gefördert wird (Siehe dazu Beispiele im Kapitel 10.2).

Herzustellen sind optimale Startbedingungen für alle Kinder. Die entscheidenden Weichen für die schulische Leistungsfähigkeit und Schulerfolge werden im frühen Kindesund Schulalter gestellt und mit zunehmender Schuldauer lassen sich Bildungsdefizite immer schlechter aufholen. Reformmaßnahmen wie die Bildung des neuen Lernbereiches „Mathematisches Tun“ im Kindergarten bieten nun eine gezielte Förderung der Kinder schon im Vorschulalter an. Viele Kantone in der Schweiz versuchen die Verbesserung der Startchancen für alle Kinder auch in dieser frühen Lebensphase anzustreben. Zum Beispiel gilt seit dem Jahre 1999 für die Kindergärten des Kantons BaselLandschaft ein Stufenlehrplan mit den verbindlichen Grobzielen (Lehrplan Kindergarten Kanton Basel-Landschaft 1999):

- Mit Mengen umgehen

- Mengenbegriffe verstehen und anwenden

- Kleine Mengen im Alltag simultan erfassen

- Einfache Muster und Reihenfolgen erfinden und nachbilden

- Zählen (1 bis 10)

- Vergleichen, sortieren, ordnen

- Mit Hohlmaßen spielerisch umgehen

- Zeitspannen erleben

- Raumvorstellung entwickeln

Derartige Lehrpläne lösen bei vielen Beobachtern und Betroffenen stärkste Bedenken aus: Kleine Kinder sollen in Zukunft sogar Mathematik betreiben, ein Fach, dem der Ruf vorauseilt, besonders schwierig und kompliziert zu sein, Misserfolge erzeugt und Ängste auslöst. Solche Befürchtungen wären dann berechtigt, wenn durch diese neuen Bildungsaufträge lediglich Inhalte und Methoden aus dem Mathematikunterricht der Primarschule in den Kindergarten vorverlegt würden. Es darf aber nicht zu einer falschen Verschulung der frühen Bildung kommen. Eine mathematische Frühförderung 
kann anspruchsvoll sein und dennoch Kindern und Kindergärtnerinnen Spaß machen und dabei die Kindheit nicht nur „unversehrt“ lassen, sondern auch durch ein weites Feld an neuen wichtigen Erfahrungen und Entdeckungen wesentlich bereichern. Der Kindergarten soll ein anregendes Betätigungsfeld auf der anschaulichen und erlebbaren Ebene bieten. Der Umgang mit mathematischen Sachverhalten (nebst den oben erwähnten) soll etwas ganz Alltägliches werden. Anzustreben ist anfänglich eine spielerische kognitive Förderung (vgl. Preiß 2002, 1; siehe dazu auch die Ausführungen im Kapitel $10 \mathrm{ab}$ Seite 210).

\subsection{Fragestellungen und Aufbau der Arbeit}

Ein Hauptgrund diese Arbeit zu verfassen, war eine Unsicherheit in der Beurteilung von Kompetenzen und Kompetenzunterschieden bei vier- bis siebenjährigen Kindern. Eine umfangreiche Kompetenzerhebung sollte diese Fragen klären. Von besonderem Interesse sind die Fragen nach den individuellen Unterschieden sowie danach, wie Kinder in diesem Alter auf konkrete mathematische Fragestellungen und Probleme reagieren. Unser Augenmerk richtet sich im Weiteren auf die Voraussetzungen, die die Kinder mitbringen müssen, um numerische und nichtnumerische Aufgaben lösen zu können. Welche Schwierigkeiten können dabei auftauchen und warum sind einige Kinder erfolgreicher als andere? Können Aussagen über die Art des Denkens gemacht werden? Zudem wurden die eruierten Daten dazu verwendet, einen Vergleich der mathematischen Kompetenz zwischen holländischen, deutschen und Schweizer Kindern durchzuführen. Diese Befragungen der Kinder wurde des Weiteren auch dazu verwendet, die Ausbildung der Lehrerinnen und Lehrer zu verbessern: Einzelinterviews mit Kindern sind überaus lehrreich und die durchführenden Studenten und Studentinnen haben enorm viel profitiert.

Um diese Fragen beantworten und die Absichten einlösen zu können, benutzten wir ein umfangreiches Testinstrumentarium: Der in Holland von van de Rijt, van Luit und von Pennings entwickelte und von Hasemann ins Deutsche übersetzte und auch in Deutschland angewendete „Utrechtse Getalbegrip Toets (UGT)“ (deutsch: Osnabrücker Test zur Zahlbegriffsentwicklung, kurz: OTZ) bietet eine gute Möglichkeit, die mathematische Kompetenz der vier- bis siebenjährigen Kinder zu testen. Die aus den umfangreichen 
Untersuchungsdaten entwickelte Kompetenzskala bietet nebst der absoluten Standortbestimmung der Kinder auch die Möglichkeit eines Vergleichs mit gleichaltrigen Kindern. Es handelt sich um halbstandardisierte Tests, wobei die Testresultate auf einer breit angelegten Untersuchung mit 832 holländischen Kindern basieren. Wie noch gezeigt wird, weichen die Ergebnisse einer Nachuntersuchung in Deutschland mit 330 und in der Schweiz mit 70 Kindern zum Teil erheblich von den Ergebnissen von Holland ab. Dies ist zum einen mit dem holländischen Bildungssystem und zum andern mit dem kulturellen Umfeld zu erklären. Solche Abweichungen lassen Rückschlüsse über die Verarbeitung von mathematischen Problemen zu.

Nach diesem Einleitungskapitel wird im zweiten Kapitel untersucht, ob die Verfügbarkeit des Zahlbegriffs nur der menschlichen Spezies vorbehalten ist. Allerneueste Forschungsergebnisse zeigen, dass auch Tiere über so etwas wie einen Zahlensinn verfügen. Davon ausgehend wird gezeigt, dass Kleinkinder auf diesem evolutionären Erbe sich schon mit kleinen Anzahlen kritisch auseinandersetzen und wohl von Anfang an über einen Zahlsinn verfügen.

Im dritten Kapitel wird die Zahlbegriffsentwicklung unser Thema sein. Auf der Basis von Piagets Entwicklungspsychologie werden verschiedene Aspekte der Entwicklung des Zahlbegriffs präsentiert.

Im vierten Kapitel wird die Bedeutung der Zählfertigkeit begründet. Das Zählen als Basiskompetenz für viele mathematische Aktivitäten ist für viele Autoren unumstößliche Tatsache.

Im fünften Kapitel wird zuerst die Frage nach der Kompetenz und Wissensverarbeitung geklärt. Worin unterscheiden sich Kompetenz und Performanz? Nach einem theoretischen Exkurs werden die situierte Kognition und die Bedeutung der mentalen Modelle eingehend untersucht.

Im sechsten Kapitel wird das benutzte Testinstrumentarium vorgestellt. Der schon erwähnte Osnabrücker Test zur Zahlbegriffsentwicklung (OTZ) wird eingehend besprochen. Zudem werden die verschiedenen Befragungsmethoden vorgestellt und miteinander verglichen.

Im siebenten Kapitel werden die Ziele und die Art der Untersuchung detailliert vorgestellt. Wie wird der OTZ eingesetzt, wie ist das Umfeld der Kindergärten und welche statistischen Werkzeuge werden verwendet. In diesem Kapitel werden dann die Ergebnisse der einzelnen Aufgaben des OTZ präsentiert und eine erste allgemeine Ergebnisanalyse erstellt. 
Im achten Kapitel werden zuerst die Leistungen der holländischen, der deutschen und der Schweizer Kinder vorgestellt und miteinander verglichen und es wird versucht, die Unterschiede zu erklären. Eine zentrale Frage der Wechselwirkung Zählkompetenz und Piaget'scher Komponenten wird beantwortet. Die im Test benutzten Piaget'schen Komponenten für den Erwerb von Zählkompetenz werden untersucht und eine Gegenüberstellung dieser Piaget'schen Komponenten mit der allgemeinen Leistungsfähigkeit der Kinder durchgeführt. Zum Schluss werden pränumerische und numerische Kompetenzen miteinander verglichen und in einem Zusammenhang gebracht.

Das neunte Kapitel zeigt die Kompetenzentwicklung und vor allem die Kompetenzunterschiede der einzelnen Kinder.

Im zehnten Kapitel wird basierend auf den im Test erbrachten Leistungen als ein Ergebnis der vorliegenden Arbeit ein klares Bekenntnis zur vorschulischen Förderung formuliert. Insbesondere werden Beispiele einer mathematischen Frühförderung im Kindergarten gegeben sowie in einem weiteren Schritt die so genannte Basisstufe - die Schweizer Variante der veränderten Schuleingangsstufe - für alle Kinder propagiert.

Im elften und zwölften Kapitel ist das Abbildungsverzeichnis, respektive das Tabellenverzeichnis untergebracht und im dreizehnten Kapitel das Literaturverzeichnis. Im Anhang werden zwei Kinder parallel durch den ganzen Test begleitet und so eine Vergleichsmöglichkeit geboten. Ebenso sind die vollständigen Testergebnisse im Anhang zu finden. 


\section{Zahlbegriff}

\section{$2.1 \quad$ Ursprünge}

Eine wichtige Grundlage der Mathematik sind ,die Zahlen, deren äußeres Gewand die Ziffern bilden.“ (Ifrah 1992, 13). Wir brauchen die Ziffern 1, 2, 3, 4, 5, 6, 7, 8, 9 und die 0 sehr vielfältig. Ob sie nun auf Kreditkarten gestanzt oder in Zeitungen die Börsenkurse angeben, ob sie das Geld auf meinem Konto oder den Rang der Fußballmannschaften in der obersten Liga dokumentieren, immer brauchen wir die 10 Ziffern. Der Gebrauch von Ziffern ist für uns Erwachsene so selbstverständlich wie essen und trinken. Wir haben vergessen, wie aufwendig und oft mühsam für viele von uns der Erwerb dieser Kompetenz im Umgang mit Ziffern und mit Zahlen war.

Es gab auch eine Zeit, in der die Menschheit nicht zählen konnte. Ifrah (1992, 17ff) berichtet von heute existierenden Volksstämmen in Afrika (Zulu, Pygmäen), Australien (Aranda, Kamilarai), Neu Guinea und im Amazonasgebiet, die nicht im Besitz der Zahlenkenntnis sind, wie wir sie kennen. Es wird beobachtet, dass im Wesentlichen eins, zwei und viele die numerischen Größen dieser Volksstämme sind, es gibt aber auch einige Menschen unter ihnen, die die Zahlen Drei und Vier bilden. Die Konstruktion dieser numerischen Größen ist aber recht primitiv: zwei-eins für Drei und zwei-zwei für Vier. Das ist keine zukunftsweisende Erfindung; denn schon höhere Zahlen sind auf diese Weise nur schwer oder überhaupt nicht zu bilden. Es besteht für viele dieser Volksstämme vermutlich kein Bedürfnis und keine Notwendigkeit, Begriffe für höhere Anzahlen zu kreieren. Falls sie aber genötigt sind, eine größere Anzahl Elemente zu beschreiben, zeigen einige von ihnen auf die Haare, so als wollten sie sagen: „Das ist so unzählbar wie die Haare auf dem Kopf“.

Es ist kaum anzunehmen, dass diese Volksstämme die Zahlen Eins, Zwei und die Beschreibung viele abstrakt erfassen. Vielmehr liegt die Vermutung nahe, dass sie die Zahl sehr sinnlich wahrnehmen. Ähnlich wie man einen Duft, eine Farbe oder ein Geräusch wahrnimmt. (Zum Beispiel kann das Flattergeräusch eines davonfliegenden Vogelschwarmes oder das Kreischen zweier kommunizierender Papageien für die Assoziationen für viele resp. zwei herhalten.) Die Zahl wird in ihrem Geist nicht losgelöst vom realen Gegenstand aufgefasst, sondern immer im Bezug zum betrachteten Gegenstand gesetzt. Es ist für diese Menschen nicht ohne weiteres einsichtig, dass zum Beispiel vier Menschen, vier Papageien, vier Trommelschläge, vier Fische oder vier Pfeile ein 
meinsames Merkmal aufweisen, das darin besteht, „Vier“ zu sein (Ifrah, 1992, 18). Diese Fähigkeit zur Verallgemeinerung ist aber eine wichtige Komponente dessen, was man mit Zahlbegriff bezeichnet. Diese Kompetenz ist bei diesen Volksstämmen zweifelsohne latent vorhanden. Zum Ausdruck kommt sie aber wohl selten oder nie. Vielmehr wird die Anzahlerfassung sinnlich bewältigt.

Häufig wird diese sinnliche Fähigkeit der Anzahlrepräsentation als direkte Zahlwahrnehmung oder einfach als Zahlengefühl bezeichnet. Dieses Zahlengefühl braucht ebenso wenig eine abstrakte Repräsentation von Zahlen wie eine Zählkompetenz.

Wir werden im nächsten Kapitel sehen, dass Kinder vermutlich mit einer ähnlichen sinnlichen Wahrnehmung der Zahlen ausgestatten sind. Das Zählen selbst baut, wie noch zu zeigen ist, auf diese sinnliche Wahrnehmung auf und bedeutet eine weitaus höhere mentale Beanspruchung des menschlichen Geistes.

Es ist gut dokumentiert, dass mit der Erfindung der Zahlen Eins und Zwei die Entwicklung ein vorläufiges Ende gefunden hat. Die Zahl Drei war seit grauer Vorzeit ein Synonym für Mehrheit, Anhäufung oder Vielheit. Dies beschreibt Georges Ifrah (1992, 20): „Im Französischen besteht ein offensichtlicher Zusammenhang zwischen dem Wort trois (drei), dem Adverb trés (sehr) - das vor einem Adjektiv oder einem Adverb dessen Intensivierung ausdrückt - und der Präposition lateinischen Ursprungs trans, die für ,jenseits, darüber hinaus“ steht. Im Altfranzösischen wurde trés als Präposition im Sinne von „,bis“ gebraucht, und das Verb transir bedeutete „sterben“ (wörtlich: „,̈berqueren, darüber hinaus gehen“).

Im Lateinischen entsprangen tres (drei) und die Vorsilbe trans demselben Stamm, und das Wort ter diente nicht nur dazu, ,drei Mal“ auszudrücken, sondern auch eine gewisse Pluralität.

Auch das englische Wort thrice hat zwei Bedeutungen: „drei Mal“ und „mehrere“. Und die Worte three (drei), throng (die Menge) und through (durch) haben eindeutig dieselbe etymologische Wurzel.

Schließlich besitzt das altsächsische Wort thria - eng verwandt sowohl mit dem englischen Wort three wie mit den germanischen Wörtern dri, drio, driu, die zum deutschen „drei“ wurden - eine gemeinsame Wurzel mit dem Wort throp (Anhäufung, Haufen), das früher im Fränkischen - der Sprache der germanischen Franken - benutzt wurde. Davon wurde einerseits das französische Adverb trop und sein italienisches Gegenstück troppo (viel, mehr als nötig) abgeleitet, andererseits - im Latein des Mittelalters - das Wort troppus (Herde, Schar), das zur Entste- 
hung der Wörter troupe und troupeau im Französischen, tropa im Spanischen, truppa im Italienischen, troop im Englischen und „Trupp“, „Truppe“ im Deutschen führte.“

\subsection{Können Tiere zählen?}

Es ist vielfach belegt, dass Tiere über etwas Ähnliches wie das oben beschriebene Zahlgefühl verfügen. Obwohl die berühmte Geschichte aus dem 18. Jahrhundert vom Schlossherrn und dem Raben vermutlich im Reich der Fabeln anzusiedeln ist ${ }^{1}$, ist „die Fähigkeit zur Diskriminierung zwischen Mengen unterschiedlicher Größen [...] kein menschliches Privileg“"(Stern 1998, 60). Es ist beeindruckend, dass einige Tiere feststellen können, ob eine Ansammlung von wenigen Gegenständen durch Hinzufügung oder Wegnahme von weiteren Gegenständen verändert wurde. Gallistel (1990), Klein und Starkey (1987) berichten über erstaunliche Fähigkeiten zur Verarbeitung quantitativer Informationen. Es wird berichtet, dass Tauben unter gewissen Umständen zwischen 45 und 50 Pickeinheiten unterscheiden können. Diese „Zählkompetenz“ wurde offenbar im Laufe der Evolution erworben und ist in ähnlicher Form bei vielen Tieren nachgewiesen worden (Stern 1998, 60). Es ist heute gut belegt, dass Ratten, Tauben, Schimpansen und andere Tiere in vielen Zusammenhängen spontan auf Anzahlen achten, dass vielen Tieren ein Sinn für Anzahlen gegeben ist.

Church und Meck (1984) haben in faszinierenden Experimenten mit Ratten gezeigt, dass diese Tiere wissen, dass $2+2=4$ ist. Ratten wurden darauf konditioniert, einen linken Hebel zu drücken, wenn sie zwei Töne hörten, und einen rechten, wenn sie vier Töne hörten. Anschließend wurde ihnen beigebracht, zwei Lichtblitze mit dem linken Hebel und vier Lichtblitze mit dem rechten Hebel zu verknüpfen. Dann stellte sich die Frage, wie diese beiden Lernerfahrungen im Rattengehirn enkodiert waren. Wurden sie als zwei zusammenhanglose Wissenseinheiten gespeichert? Oder hatten die Ratten eine

\footnotetext{
${ }^{1}$ Ein Schlossherr wollte einen Raben töten, der im Wachturm des Schlosses nistete, und hatte mehrmals versucht, den Vogel zu überraschen. Aber jedes Mal, wenn er sich dem Nest näherte, flog der Rabe weg und ließ sich außer Schussweite in einem benachbarten Baum nieder, kam jedoch zurück, sobald sein Verfolger den Turm wieder verlassen hatte. Der Schlossherr griff daraufhin zu einer List: Er ließ zwei seiner Begleiter in den Turm ein; nach wenigen Minuten zog sich der eine zurück, während der andere blieb. Der Rabe aber ließ sich nicht überlisten und wartete das Verschwinden des zweiten ab, bevor er an seinen alten Platz zurückkehrte. Auch drei und vier Männer konnten das kluge Tier nicht überlisten, denn jedes Mal wartete es, bis alle Jäger den Turm verlassen hatten. Das Experiment gelang schließlich mit fünf Personen, denn als vier von ihnen den Turm verlassen hatten, kam der doch nicht so zahlenkundige Rabe zurück und wurde vom fünften Jäger erlegt (vgl. Ifrah 1989, 21).
} 
abstrakte Regel wie 2 ist links und 4 ist rechts gelernt? Um das herauszufinden, präsentierten die Forscher bei einigen Versuchen sowohl Töne als auch Lichtblitze und beobachteten erstaunt, dass die Ratten dann, wenn sie einen einzelnen mit einem Blitz synchronisierten Ton hörten, insgesamt also zwei Ereignisse, systematisch den linken Hebel drückten. Umgekehrt drückten die Ratten sofort den rechten Hebel, wenn ihnen eine Folge von zwei Tönen in Verbindung mit zwei Lichtblitzen präsentiert wurde, also insgesamt vier Ereignisse vorlagen. Die Tiere verallgemeinerten ihr Wissen damit auf eine völlig neue Situation. Ihre Begriffe der Zahlen 2 und 4 waren nicht an eine basale Schicht der visuellen oder auditiven Wahrnehmung gekoppelt.

Um die Bedeutung dieses Befundes besser zu erfassen, vergleiche man ihn mit einem Gedankenexperiment, bei dem eine Ratte lernt, den linken Hebel sowohl dann zu drücken, wenn sie ein Quadrat (und nicht einen Kreis) sieht, als auch dann, wenn sie die Farbe Rot sieht (und nicht Grün). Wenn dieser Ratte ein rotes Quadrat vorgelegt würde - eine Kombination beider Reize -, würde sie doch sicherlich noch entschiedener auf den linken Hebel drücken. Warum gilt nicht das gleiche für die Zahl der Töne und Lichtblitze? Der Versuch beweist, dass Ratten bis zu einem gewissen Grad wissen, dass Zahlen sich nicht so addieren wie Formen und Farben. Ein Quadrat plus die Farbe Rot ergibt ein rotes Quadrat, aber zwei Töne plus zwei Blitze führen nicht zu einem noch größeren Gefühl von Zweiheit. Vielmehr sind $2+2$ gleich 4, und das Rattengehirn hat anscheinend einen Sinn für Arithmetik (siehe auch Dehaene 1999, 31).

Beachtung gefunden haben auch die Arbeiten von Woodruff und Premack (1981), in denen sie zeigten, dass Schimpansen schon Ansätze zur Bewältigung von einfachen Additionsaufgaben zeigen. Die Autoren haben glaubhaft demonstriert, dass diese Tiere spontan die Summe von 4 und 3 ermittelten ebenso wie die Summe von 5 und 1. Darauf stellten die Schimpansen fest, dass 7 größer ist als 6.

Bei allen solchen Versuchen kommt zum Ausdruck, dass Tiere vermutlich über einen „Schätzalgorithmus“ verfügen. Sie besitzen sicher keine diskrete Repräsentation von Zahlen. Außer den ersten Zahlen 1, 2 und 3 wird wohl alles verschwommen. Es ist für Tiere umso schwerer $n$ von $n+1$ zu unterscheiden, je größer $n$ wird. Je größer $n$, desto größer muss der Abstand zwischen den Repräsentanten der zu unterscheidenden Zahlen sein. 3 kann von 4 in einigen ausgewählten Experimenten von Schimpansen unterschieden werden, nicht aber 7 von 8 (Woodruff und Premack 1981 in Dehaene 1999, 34ff). Offenbar spielt der prozentuale Unterschied zwischen den zu vergleichenden Mächtigkeiten die ausschlaggebende Rolle. 
Es ist anzunehmen, dass dieser Vergleichsalgorithmus für die Erhaltung der Art von Bedeutung ist. Diesen Vergleichsalgorithmus besitzen wohl auch einfachste Organismen, denn man „,muss optimieren, um überleben und vergleichen, um optimieren zu können “ (Dehaene 1999, 39).

Die Ergebnisse vieler weiterer Versuche lassen vermuten, dass Zählen und Rechnen im weitesten Sinn nicht eine Fähigkeit ist, die nur Menschen besitzen, sondern im Tierreich ebenfalls vorkommt. Es ist oft feststellbar, dass solche Vermutungen Kopfschütteln, Irritationen und Befremden auslösen; denn Tiere verfügen über keine Sprache und nach gängiger Meinung ist zur Bewältigung von Mathematik Sprachkompetenz unbedingt erforderlich.

Dehaene schlägt auf der Grundlage von Arbeiten von Meck \& Church (1983) zur Erklärung dieses Phänomens ein Akkumulatormechanismus vor. Dieser Mechanismus erlaubt es vielen Tieren, die Kardinalzahl einer Menge (die Mächtigkeit einer Menge) genauso einfach wahrzunehmen wie ihre Farbe, Form oder Lage.

Diese Theorie versucht zu erklären, wie die erstaunlichen numerischen Kompetenzen der Tiere ohne Sprachkompetenz möglich werden. Das Nervensystem der Ratten und Schimpansen ist kaum dazu in der Lage, diskrete Dinge exakt zu zählen. Das Speichermodell hilft uns zu verstehen, wie Tiere in der Lage sind, numerische Quantitäten wahrzunehmen resp. Mächtigkeiten von Mengen zu unterscheiden. Man denke sich den Speicher als Wasserbehälter und jedes hinzugefügte Element hebt den Wasserspiegel. Jedes zu zählende Element wird in diesen Speicher gelegt. Nicht als Einzelelement, sondern als stetige Größe, eben vergleichbar mit Hinzufügen von Wasser. Je mehr Elemente erfasst werden, desto höher der Wasserspiegel. Dieses Akkumulatorprinzip erlaubt es nun auf einfache Art, zwei Mengen zu vergleichen und zu erkennen, wie eine Mächtigkeit sich verändert. Dieses Modell erklärt unter anderem auch die Ungenauigkeiten bei größeren Zahlen. Die Tiere rechnen nicht eigentlich, sondern sie schätzen ab. Sie schätzen die Anzahl auf der Grundlage des mentalen Bildes des „Wasserstandes“ und können keine genaue Anzahl bestimmen, Tiere haben so nur die Möglichkeit zu unscharfem „Zählen“. Dies ist ein großer Unterschied zu unserem gewohnten Zählen; wir zählen mit diskreten Einheiten und erfassen die Anzahl exakt. Jedem gezählten Element entspricht genau ein Schritt in der Zahlenfolge. Nicht so bei Tieren. Ihr „Zählen“ entspricht eher dem wechselnden Stand im „Wasserbehälter“. Dieser „Wasserstand“ ist eine analoge Repräsentation der Anzahl und weit entfernt von unserem Zahlbegriff. 
Wir sind Kinder der Evolution. Verfügen Schimpansen über numerische Kompetenzen und sind diese Fähigkeiten auch verschiedenen anderen Tierarten wie Ratten und Vögel nicht fremd, so liegt der Schluss nahe, dass unser Menschengehirn über einen ähnlichen Speicher verfügt, um numerische Größen wahrzunehmen, zu speichern und zu vergleichen (vgl. Dehaene 1999, 34-46).

Beobachtungen bei kleinen Kindern lassen tatsächlich die Vermutung aufkommen, dass junge Menschenkinder eine ähnliche Repräsentation der numerischen Umwelt in ihrem Nervensystem ausweisen.

\subsection{Kinder haben einen Zahlensinn}

In der kognitiven Entwicklungspsychologie wurde viele Jahre die These vom Säugling als kognitives Tabula-Rasa-Wesen propagiert. Das neugeborene Kind sei ausgestattet lediglich mit dem überlebenswichtigen Saugreflex und der Fähigkeit, Informationen zu verarbeiten und zu lernen. Insbesondere die Akkommodation, Assimilation und Äquilibration (in der Sprache des Entwicklungspsychologen Piaget) und die Fähigkeit, ReizReaktions-Verbindungen aufzubauen (in der Sprache des Behavioristen Watson), biete Gewähr dafür, dass Menschen sich entwickeln können (vgl. Stern 1998, 46).

Viele Indizien sprechen aber dafür, dass die Menschen von Geburt an mit weiteren (anderen) Fähigkeiten ausgestattet sind. Insbesondere wird von vielen Autoren angenommen (Dehaene 1999, 76ff; Stern 1998, 59ff; Starkey, Spelke und Gelman 1990 u.a.), dass das menschliche Gehirn vermutlich mit einem im Laufe der Evolution erworben angeborenen Mechanismus ausgestattet ist, der das Erfassen numerischer Größen erlaubt. Ist ein numerisches Konzept Voraussetzung für das Erlernen der Zahlwörter in der Phase des Spracherwerbes, das mit ungefähr eineinhalb Jahren beginnt? Die direkt betroffenen Kleinkinder können unsere Fragen nicht beantworten. Es ist nicht möglich, direkt zu erfahren, wie die Wahrnehmung von Säuglingen funktioniert, wie Kinder denken.

Zugang zum frühen Wahrnehmungsrepertoire fanden aber Entwicklungspsychologen in den sechziger Jahren. Damals erkannten sie, dass Säuglinge Objekte, die neu für sie sind oder die sie irritieren, deutlich länger betrachten. Blickzeiten zu messen hat sich seither als Erkenntnisschlüssel erwiesen. Dieser indirekte Weg eröffnet Möglichkeiten, auch numerische Kompetenzen der Kleinkinder zu erforschen. Es gibt also eine Möglichkeit 
herauszufinden, ob noch nicht einjährige Kinder schon Aspekte des Zahlbegriffs beherrschen, ohne ihn aus Wechselwirkung mit der Umwelt herzuleiten.

Durch einen berühmt gewordenen Versuch konnten P. Starkey, E. Spelke und R. Gelman (1990) nachweisen, dass Säuglinge im Alter zwischen sechzehn und dreißig Wochen zwischen 2 und 3 unterscheiden können. Dadurch kamen sie zum Schluss, dass Kleinkinder Anzahlen wahrnehmen. ${ }^{2}$ Weitere ähnliche Versuche mit vergleichbaren Ergebnissen wurden mit noch jüngeren Kindern durchgeführt (Antell \& Keating 1983). Vielleicht ermöglicht eine innere, abstrakte Repräsentation von Anzahlen dem Kind, die Übereinstimmung zwischen der Anzahl von Objekten herzustellen. Vielleicht ist aber auch das frühreife visuelle System (vergleichbar mit einer inneren „Photographie“) von Kleinkindern so leistungsfähig, dass auf diesem Weg eine Anzahl erkannt wird. Dieser intuitive Zahlensinn wird durch neuste Erkenntnisse der Hirnforschung aufgewertet. In einem im Fachblatt „Science“ (Science 284, 970-974) veröffentlichten Artikel berichten S. Dehaene und E. Spelke, dass die bildhafte Vorstellung von Zahlen und die abstrakte Fähigkeit, drei plus fünf zu addieren, vermutlich zwei völlig unterschiedliche Gebiete im menschlichen Gehirn beanspruchen.

Doch erst die Kombination der beiden Zahlenmodule macht uns zur rechnenden Spezies. Es gibt verschiedene Arten, mathematische Probleme zu bearbeiten. Viele Mathematiker setzten die Probleme in eigene Bilder um, mit denen sie weiterarbeiten. Ein Albert Einstein zugesprochenes Zitat soll diese Art Mathematik zu betreiben erläutern: „Wenn es um Mathematik geht, scheinen Worte und Zahlen bei mir keine Rolle zu spielen. Vielmehr dienen mir mehr oder weniger deutliche Bilder, die ich beliebig abrufen, verändern und kombinieren kann, als Bausteine für die Gedankengänge." Von anderen Mathematikern hingegen ist bekannt, dass sie sich beim Lösen rechnerischer Knacknüsse voll auf Wörter und Sprache verlassen. Was ist nun effektiver (vgl. auch Käpnick 1998, 260ff und Rickmeyer 2001, 51ff)? Käpnick (1999) hat in einer Studie zeigen kön-

\footnotetext{
${ }^{2}$ Einem Baby, das vor zwei Leinwänden sitzt, werden Dias gezeigt. Gleichzeitig eines rechts und eines links. Auf dem rechten Dia werden zwei alltägliche Dinge gezeigt, die beliebig angeordnet sind. Auf dem linken Dia werden drei ähnliche Gegenstände ebenfalls in beliebiger Anordnung gezeigt. Während des ganzen Experimentes wird das Baby mit einer verborgenen Videokamera beobachtet und die Blickzeiten gemessen. Dadurch konnte gemessen werden, wie viel Zeit das Kind beim Betrachten der einzelnen Bilder verbringt. Nach einer gewissen Zeit lässt das Interesse der Kinder nach den Bildern nach. In dieser Phase werden Trommelschläge aktiviert und es ergibt sich eine interessante Feststellung: Der Blick des Babys ist länger auf das Dia gelenkt, das genauso viele Dinge zeigt, wie das Kind Töne hört. Es schaut länger auf das Bild mit drei Objekten, wenn es drei Töne hört und länger auf das Bild mit zwei Dingen, wenn es zwei Töne hört. Es kann sein, dass das Baby die Anzahl der Töne erkennt und sie mit der Anzahl der Gegenstände auf den Dias vergleicht. Kann es sein, dass das Kind wirklich Anzahlen wahrnimmt und nicht nur ein akustisches Signal oder eine geometrische Anordnung? Falls das zutrifft, wird im Gehirn des Kindes wohl dieselbe Repräsentation der Zahl 3 aktiviert, ob es nun drei Töne hört oder drei Dinge sieht.
} 
nen, dass subjektive Zahlauffassungen bei Kindern relativ häufig vorkommen, sehr vielfältig sein können und dass solche Auffassungen das Erlernen des Zahlbegriffs beeinflussen können. Als Beispiel einer fast animistisch anmutenden Auffassung: „Zahlen gehören immer in Gruppen zusammen. Aber große Zahlen sind stark, die können alleine stehen. Kleine Zahlen sind schwach und brauchen noch Hilfe.“ (Josephine, 1. K1. in Käpnick 1999, 275). Käpnick berichtet von einem ,inneren“ Kampf zwischen einer „offiziell richtigen“ Zahlanordnung und den eigenen andersgearteten persönlichen subjektiven Zahlanordnungen, den einige Kinder ausfechten. Solche subjektive Zahlauffassungen können nach Käpnick die Motivation beim Lernen im Mathematikunterricht beeinflussen. Der Autor erwähnt das Verwenden von persönlichen „Lieblings-“ resp. „Pechzahlen“, was motivationsfördernd, resp. motivationshemmend sein kann. Für leistungsschwache Kinder bedeutet der Umgang mit subjektiven Zahlauffassungen eine zusätzliche Schwierigkeit beim Erlernen von Mathematik. Interessant scheint mir aber, dass auch leistungsstarke Kinder „,mathematik-untypische“ Zahlanordnungen verwenden und durchaus souverän zwischen ihren subjektiven Auffassungen und den „offiziell richtigen“ unterscheiden können. „Sie ,spielen’ sogar gern mit ihren individuellen ,Zahlenbildern', nutzen diese oft für vorteilhaftes Rechnen, oder solche andersartigen Auffassungen sind Auslöser für originelle Lösungsideen (kreativitätsfördernde Funktion subjektiver Zahlauffassung)“ (Käpnick 1999, 276; Anführungszeichen und Klammer im Original).

Vielleicht benötigen wir tatsächlich zwei unterschiedliche Gehirnbereiche für die verschiedenen Arten der Repräsentation. Die unterschiedlichen Denkweisen scheinen nämlich nur auf den ersten Blick unvereinbar; sie sind es aber durchaus, wie S. Dehaene und E. Spelke zeigen konnten. Das Gehirn benützt für die Bewältigung mathematischer Aufgaben zwei verschiedene Bereiche: Das eine Areal führt exakte Berechnungen durch und gibt den Zahlen Namen, ist also mit Sprache verknüpft. Im anderen Areal sitzt der eher intuitive Zahlensinn, eine Art mentale Zahlenreihe oder Akkumulator, mit dem wir Größenordnungen abschätzen können. Weitere Untersuchungen müssten durchgeführt werden, um die Frage nach einem Zusammenhang zwischen diesem intuitiven Zahlensinn und dem oben beschriebenen Akkumulatormechanismus zu klären.

Hinweise darauf, dass verschiedene Hirnbereiche bei der Lösung von Mathematikproblemen beansprucht werden, häuften sich in letzter Zeit. So können zum Beispiel gewisse Patienten nach einem Hirnschlag nicht mehr entscheiden, ob 9 näher bei 10 oder 5 liegt, und dies, obwohl ihnen einfache Additionen wie $7+3=10$ überhaupt keine Probleme 
bereiten. Andere Patienten wiederum können sich nicht entscheiden, ob $2+2$ eher 3 oder 4 ergibt. Werden sie allerdings gefragt, ob sie als Resultat eher 3 oder 9 vorziehen würden, wählen alle die 3.

Damit war für Dehaene eines klar: Bei der Bewältigung mathematischer Probleme benützt das Hirn mindestens zwei verschiedene Bereiche. Um diese Hypothese zu testen, rekrutierten Dehaene mit der Psychologin Elizabeth Spelke zweisprachige CollegeStudenten.

In einem ersten Experiment mussten die fließend Englisch und Russisch sprechenden Studenten einfache Rechenaufgaben lösen: „Ist 53 plus 68 gleich 121 oder 127?“, eine Aufgabe, die eine exakte Kalkulation erforderte. Eine Schätzung war hingegen bei der Frage gefordert, ob das Ergebnis von 53 plus 68 eher bei 120 oder 150 liegt.

Die Schwierigkeit des Tests war versteckt: Spelke instruierte die Studenten entweder auf Englisch oder Russisch und testete sie danach in derselben oder in der anderen Sprache. Keine Rolle spielte die Sprache bei der zweiten Kategorie von Aufgaben, wo die Studenten nur abschätzen mussten, welche Antwort zutrifft: Egal, ob der Test in der gleichen oder in der anderen Sprache wie die Instruktion durchgeführt wurde, brauchten die Studenten etwa gleich lang für die Lösung.

Die Überraschung kam indes bei den exakten Aufgaben. Wurden die Studenten auf Englisch instruiert und auf Englisch getestet, antworteten sie rund eine Sekunde schneller, als wenn die Sprache gewechselt wurde - ein Resultat, das an Klarheit selbst die Forscher überraschte.

Auch als die Forscher den Schwierigkeitsgrad der Aufgaben erhöhten, änderte sich nichts: Mussten die Studenten das Resultat einer Wurzelgleichung nur abschätzen, spielte die Sprache keine Rolle; bei komplizierten exakten Additionen hingegen war die Sprache der entscheidende Faktor, wie lange sie zur Lösung brauchten.

Mit anderen Worten: Das Lösen von exakten Aufgaben scheint eng mit sprachlichen Fähigkeiten gekoppelt, weil die Studenten zuerst von Englisch auf Russisch (oder umgekehrt) übersetzen mussten, um das Problem zu lösen. Andererseits ist die Fähigkeit, Größenordnungen zu schätzen, unabhängig von der Sprache.

Um diese These zu beweisen, durchleuchtete Dehaene in einem zweiten Experiment die rechnenden Hirne von Freiwilligen mit einem Kernspintomographen. Mussten die Studenten exakte Additionen ausführen, war eine Hirnregion im linken Stirnlappen besonders aktiv - dort, wo das Hirn auch Tätigkeits- und Hauptwörter verarbeitet. Hatten die Freiwilligen hingegen eine Schätzaufgabe zu lösen, leuchtete in beiden Hirnhälften eine 
Region im Scheitellappen auf. Diese Areale benützt das Hirn für räumliche und visuelle Aufgaben, dort rotiert es geistig Objekte, lenkt es die Aufmerksamkeit, und ebenfalls in dieser Region werden Finger- und Augenbewegungen gesteuert.

Dass Fingerbewegungen und der nichtsprachliche Zahlensinn am gleichen Ort gespeichert sind, ist vielleicht die Erklärung dafür, dass Kleinkinder schon ab einem Alter von sechs Monaten ihre Finger benutzen, um Änderungen in der Anzahl von Objekten festzustellen. Diese Fingertechnik ist sehr effizient; denn Vorschulkinder sowie Schülerinnen und Schüler (1. Schuljahr) weiten diese Hilfe der Anzahlerfassung aus zum exakten Rechnen.

Es verdichten sich die Indizien, dass wir mit guten Grund sagen können, dass die Kleinkinder einen Zahlensinn haben, lange bevor sie sprechen können, und dass sie in dieser Phase den Tieren ähnlich sind, die solche simplen „Rechnungen“ ebenfalls beherrschen. Mit dem Sprechenlernen kommt beim Kind das exakte Rechenmodul hinzu, das sich der Mensch im Verlauf der Evolution mit der Sprache angeeignet hat. Erst die Kombination der beiden, davon ist Dehaene überzeugt, gaben dem Menschen schließlich die bemerkenswerte Fähigkeit, mit Zahlen zu jonglieren und komplizierte mathematische Probleme zu lösen.

Diese spannenden Erkenntnisse von Dehaene und Spelke könnten auch den Mathematikunterricht beeinflussen. Nicht nur die sprachabhängige Förderung sollte Bestandteil des Mathematikunterrichtes sein, sondern auch auf die mathematische Intuition müsste mehr Wert gelegt werden (siehe dazu Carraher, Carraher und Schliemann 1985). ${ }^{3}$

Schon früher hat Karen Wynn (1992b) aufsehenerregende Versuche mit Babys durchgeführt, um weitere Fragen der frühkindlichen Mathematik zu klären. Wieder mit Messung von Aufmerksamzeiten scheint es K. Wynn gelungen zu sein zu zeigen, dass Säuglinge von viereinhalb Monaten in der Lage sind zu entscheiden, dass $1+1$ nicht 3 oder 1 ist, sondern genau 2. Die oben beschriebenen Befunde von Dehaene und Spelke rücken die Resultate von Wynns Versuchen in ein klareres Licht. Es scheint, dass es sich bei diesen Fähigkeiten der Kleinkinder nicht um numerisches Wissen, sondern eher um das leistungsfähige visuelles System handelt, das die Kinder zu diesen eindeutigen Schlüssen führt. Ich komme im Kapital Zählkompetenz ab Seite 42 darauf zurück.

\footnotetext{
${ }^{3}$ Darin wird von den sehr beeindruckenden Ergebnissen der Untersuchungen zur so genannten Strassenmathematik in Brasilien berichtet. Rückschlüsse auf den Unterricht oder kindgemässes Lernen lassen diese Untersuchungen allerdings nur bedingt zu (Franke 1998).
} 
Mit dieser knappen Zusammenstellung wollte ich aufzeigen, dass kleine Kinder vermutlich von Natur aus befähigt sind, Anzahlen zu erfassen. Die Versuche von Dehaene und Spelke sowie Wynn lassen den Befund zu, dass ein mentales Modul für die Zahlwahrnehmung existiert. Alles deutet darauf hin, dass Babys über etwas Ähnliches wie den Akkumulatormechanismus verfügen, der als Erklärungsversuch für die numerischen Fähigkeiten bei Tieren diente.

Es ist aber durchaus möglich, dass die Kleinkinder über die gleichen sinnlichen Fähigkeiten der Anzahlrepräsentation als direkte Zahlwahrnehmung oder einfach als Zahlengefühl wie die oben beschriebenen Naturvölker verfügen. 


\section{$3 \quad$ Zahlbegriffsentwicklung}

\subsection{Geschichtliches und Fachliches zur Entwicklung des Zahlbegriffs}

Die Gebiete und Inhalte der Mathematik sind abstrakter als die in anderen Wissenschaften. Selbst bei den natürlichen Zahlen, eine der Basiseinheiten der Mathematik, handelt es sich um eine abstrakte Konstruktion. Auf die Frage, was denn eine Zahl sei, kann man z.B. angeben ,5“, auf fünf Gegenstände zeigen oder etwas über die Funktion von Zahlen aussagen, wie z.B. „man kann damit zählen“ oder „damit kann man die Anzahl von Gegenständen ermitteln“. Es ist aber nicht möglich einem Menschen, der nicht über einen Zahlbegriff verfügt, die Bedeutung der Zahl „5“ näher zu bringen; weder durch Zeigen auf fünf Objekte noch mit dem Aufzeichnen des Zahlsymbols „5“ (Stern 1998, 55). Diese Frage, was denn eine Zahl sei, oder die Frage nach dem Wesen der Zahl beschäftigt die Mathematiker und Philosophen seit Jahrtausenden. Es existiert eine ganze Reihe von Theorien und entsprechende Literatur zu diesem Thema (Beth; Piaget 1966; Brainerd 1979; Ifrah 1989 und 1992; Kline 1990). Eine äußerst interessante Frage, die die Philosophen aber auch Mathematiker immer wieder beschäftigte, ist die nach dem Ursprung der Zahlen. ,Sind die Zahlen in den Bau der Natur eingewoben, kommt ihnen also eine vom Wahrnehmen und Denken des Menschen unabhängige Existenz zu?“ (Maier 1990, 6). Prominente Vertreter dieser Auffassung waren die Pythagoräer. Sie betrachteten die Zahlen als den Kern und das Wesen der Dinge. Aristoteles fasst das mathematische Weltbild der Pythagoreer wie folgt zusammen: „In dieser Zeit [...] beschäftigten sich die sogenannten Pythagoreer als erste mit der Mathematik, bauten sie weiter aus und waren, da sie sich sehr mit ihr auseinandergesetzt hatten, der Meinung, dass in ihren Prinzipien die Prinzipien der Dinge gelegen seien. Da nun von diesen Prinzipien die Zahlen von Natur aus das Erste sind, sie aber in diesen gerade viele Ähnlichkeiten mit dem Seienden und Entstehenden zu sehen meinten [...], weil sie also glaubten, alle anderen Dinge glichen ihrer ganzen Natur nach den Zahlen [...], nahmen sie an, dass die Elemente der Zahlen die Elemente aller Dinge seien und der gesamte Himmel sei Harmonie und Zahl“ (Aristoteles in Schwarz 1970, 30). Als Beweis verwiesen sie zum Beispiel auf das Phänomen, dass sich die Tonintervalle durch Unterteilung einer Monokordseite erzeugen lassen, deren Länge zu den Teilintervallen in einem ganzzahligen Verhältnis steht (Oktave 2:1, die Quinte 2:3, die Quarte 3:4, große Septime 15:8, kleine Septime 9:5 usw.). Die heilige Zahl 10 und ihre Proportionen gelten zugleich als Grund- 
verhältnis des gesamten Kosmos, der sich aus fünf Gegensatzpaaren aufbaut. Fast zweitausend Jahre später griff Johannes Kepler (1571 - 1630) in seinem Werk „Mysterium cosmographicum“ 1596 den Gedanken einer durch die Zahl geordneten Weltharmonie wieder auf. Er versuchte, die Abstände im Bahnsystem der Planeten nach ganzen Zahlverhältnissen zu ordnen und dadurch die Harmonie des Sonnensystems zu beweisen. Kepler sprach von „heiliger Raserei und einer unsagbarer Verzückung“ beim Anblick der himmlischen Harmonien. In seiner Weltharmonik (Marmonices mundi) von 1609 verschmelzen exakteste mathematische Berechnungen mit zum Teil oben geschilderten uralten pythagoreischen Vorstellungen, dass die Bewegung der Gestirne eine unhörbare, aber als mathematische Proportion erfassbare Sphärenharmonie erzeugt (vgl. Helferich 1998, 141).

Die andere Auffassung, dass die Zahl nur im menschlichen Denken existiere, gewissermaßen erst durch den Menschen selber, wurde vor allem durch Platon vertreten. Er stellte die Zahlen ins Reich der Ideen, betrachtete sie den Menschen eingeboren. Für Immanuel Kant (1724 - 1804) sind die Zahlen den Menschen a priori verfügbar und er braucht sie dann nur noch auf Erscheinungen in der Wirklichkeit anzuwenden. In dieser Ideenwelt befindet sich auch Leopold Kronecker $(1823$ - 1891) der den berühmten Satz prägte: „Die natürlichen Zahlen sind vom lieben Gott geschaffen; alles andere ist Menschenwerk.“

Im Gegensatz dazu war für die empirische Erkenntnistheorie die Zahl Produkt einer ausschließlich aus sinnlicher Wahrnehmung gewonnen Vorstellung. Ideen sind hier nur durch Abstraktion verblasste Abbilder häufiger Sinneseindrücke. Vertreter dieser Auffassung waren u.a. John Locke (1632 - 1704) sowie John Stuart Mill (1806 - 1873).

Im Allgemeinen war es aber während der ganzen Menschheitsgeschichte wohl so, dass für diejenigen, die mit Zahlen umgehen konnten, auch die Begriffsbildung abgeschlossen und gefestigt. In diesem Sinne konnten Mathematiker und Naturphilosophen mit einem intuitiven Verständnis der Zahlen auf dem Gebiet der Mathematik und in den übrigen Wissenschaften Hervorragendes leisten. Für die Mathematiker was die Frage nach dem Wesen der Zahl für lange Zeit uninteressant. Sogar die Mathematiker der Neuzeit beantworteten die Frage nicht. Für Dedekind (1831 - 1916) war die Zahl eine „freie Schöpfung des menschlichen Geistes“. Im Zuge der Schaffung der Grundlagen der Mathematik im 19. Jahrhundert hat Giuseppe Peano (1858 - 1932) fünf Axiome zur 
Definition der natürlichen Zahlen formuliert. ${ }^{4}$ Eigenschaften der Zahlen dienten zur Definition. Peano geht dabei von einem ordinalen Ansatz aus. Bertrand Russell (1872 1970) und Alfred N. Whitehead (1861 - 1947) haben in ihrem epochemachenden Werk Principia Mathematica versucht zu zeigen, dass sich Zahlen allein auf logische Operationen zurückführen lassen. Dabei wählten sie einen kardinalen Ansatz. Vor allem durch Anregung von David Hilbert (1862 - 1943) wurden die übrigen Zahlen (ganze, rationale, irrationale und komplexe Zahlen) in formalistischer Weise mittels eines Systems von Eigenschaften axiomatisch definiert (vgl. Maier 1990, 6).

Es ist interessant und höchst bezeichnend, „,..., dass die logischen Grundlagen des Zahlensystems, der Algebra und der Analysis erst gegen Ende des 19. Jahrhunderts entstanden sind. Mit anderen Worten: Während der Jahrhunderte, in denen die Hauptgebiete der Mathematik entwickelt wurden, gab es für die meisten von ihnen [den logischen Grundlagen, F.C.] keine große Entwicklung. Offenbar sind die Intuitionen großer Männer mächtiger als Logik. [...] Nicht die Logik bewog schließlich die Mathematiker, diese Konzepte einzuführen, sondern es waren Argumente der Analogie, der physikalischen Bedeutung einiger Begriffe sowie das Erzielen handfester naturwissenschaftlicher Ergebnisse. Mit anderen Worten, es war die anschauliche Evidenz, welche die Mathematiker diese Grundbegriffe akzeptieren ließ. Die Logik folgte stets in weitem Abstand und war ganz offenbar schwerer zugänglich“ (Kline 1974, 56 in Moser Opitz 2001, 16).

Im Zahlbegriff sind verschiedene Aspekte integriert, die bei einer umfassenden Zahlbegriffskompetenz beherrscht werden. Diese Aspekte entsprechen den verschiedenen Verwendungen der Zahlen. Es folgt eine Auflistung dieser Aspekte:

- Kardinalzahlaspekt. Diese Zahlen beschreiben die Mächtigkeit von Mengen, die Anzahl der Elemente.

- Ordinalzahlaspekt. Hier wird unterschieden einerseits zwischen

○ Zählzahl als Folge der natürlichen Zahlen, die beim Zählen durchlaufen werden und der

○ Ordnungszahl. Diese gibt den Rangplatz eines Elementes in einer total geordneten Reihe an.

\footnotetext{
4 1. 1 ist eine natürliche Zahl.

2. Zu jeder natürlichen Zahl $n$ existiert genau ein Nachfolger $n$ ', der ebenfalls der natürlichen Zahlenmenge angehört.

3. Es gibt keine natürliche Zahl, deren Nachfolger 1 ist.

4. Die Nachfolger zweier verschiedener natürlichen Zahlen sind voneinander verschieden.

5. Eine Menge von natürlichen Zahlen enthält alle natürlichen Zahlen, wenn 1 zur Menge gehört und mit einer natürlichen Zahl $n$ stets auch der Nachfolger n’ zur Menge gehört.
} 
- Maßzahlaspekt. Natürliche Zahlen dienen in Relation zu einer gewählten Einheit als Maßzahlen für Größen.

- Operatoraspekt. Zahlen werden zur Bezeichnung einer Vielfachheit einer Handlung oder eines Vorganges benutzt.

- Rechenzahlaspekt. Es wird unterschieden zwischen dem

- Algebraischen Aspekt, indem die natürlichen Zahlen verknüpft mit der Addition eine algebraische Struktur mit gewissen Eigenschaften aufweist und dem

○ Algorithmischen Aspekt. Die natürlichen Zahlen lassen sich durch Ziffernreihen darstellen. (Rechen mit Ziffern)

- Codierungsaspekt. Zahlen werden zur Bezeichnung von Objekten benutzt.

(Radatz, Schipper 1983, 49)

Es ist anzunehmen, dass bei dieser Komplexität des Zahlbegriffs die Kinder die verschiedenen Zahlbedeutungen einzeln erwerben. „Erst im Laufe der Schulzeit werden die Wechselbeziehungen zwischen den verschiedenen Zahlaspekten deutlich und damit ein umfassender Zahlbegriff erarbeitet" (Radatz, Schipper 1983, 49; vgl. auch Bauersfeld 1983). Für eine umfassende Untersuchung der Genese des Zahlbegriffes heißt das also, der Entwicklung der einzelnen Aspekte und ihrer progressiven Koordinierung nachzugehen. „Vom operativen Standpunkt aus genügt es dabei natürlich nicht zu untersuchen, inwieweit Kinder wissen, wie groß die Anzahl der Elemente einer Menge, welches der Rangplatz eines Elementes in einer Reihe, welches das Ergebnis einer Rechnung ist usw., sondern es muss auch gefragt werden, inwieweit sie überblicken, wie sich Anzahl, Rangplatz, Rechenergebnis usw. bei Transformationen ändern. Interessant ist also auch die Koordination der Zahlaspekte mit operativen Schemata wie Erweitern/Reduzieren einer Menge, Verlängern/Verkürzen/Umordnen einer Reihe, Vergrößerung/Verkleinerung eines Summanden oder eines Faktors in einer Rechnung usw.“ (Wittmann 1982, 43).

Wie entwickelt sich nun beim Menschen und insbesondere beim Kind ein Zahlbegriff? Dies ist im weitesten Sinn eine Fragestellung der Psychologie und auch darum hat sich die Mathematik selbst diese Frage eigentlich nie gestellt. Erst mit dem Aufkommen der Psychologie als Wissenschaft wurde diese für uns zentrale Frage überhaupt erst gestellt. „Es interessierte nicht mehr in erster Linie eine logische Begründung der Zahl, sondern 
die Frage, wie der Zahlbegriff erworben wird und wie Zahlen im menschlichen Denken und beim Problemlösen verwendet werden. Ursprünglich logisch begründete Konzeptionen wurden mit der Zeit immer mehr auch unter psychologischen Gesichtspunkten betrachtet" (Moser Opitz 2001, 16).

Der Zahlbegriff muss also auch als komplexes Schema im psychologischen Sinne aufgefasst werden. Wir beschränken uns in dieser Arbeit nur auf die Anfänge des Zahlbegriffserwerbs, d.h. wir untersuchen Kinder bis zum Alter von ungefähr sieben Jahren. Als Grundlage der weiteren Erkundung des Zahlbegriffserwerbs berufen wir uns auf die Stufen der kognitiven Entwicklung von Jean Piaget. Seine Sicht soll uns gewissermaßen als Raster dienen. Zudem postulierte Jean Piaget prinzipiell gleiche Mechanismen in der Wissenschaftsgenese und der Psychogenese. So hat er versucht, den logischen und psychologischen Zugang zur Zahl zusammenzuführen. Um auch diesen Zugang umfassend würdigen zu können, folgt eine kleine Zusammenstellung der psychologischen und kognitiven Strukturen nach Piaget.

\subsection{Kognitive Strukturen nach Piaget}

Als Grundeinheiten der psychischen Entwicklung werden nach Piaget in Abgrenzung zu den behavioristisch orientierten Lerntheorien nicht isolierte Elemente des Verhaltens, sondern Strukturen angenommen. „Jede Entstehung geht von einer Struktur aus und mündet in eine Struktur“. „Doch umgekehrt hat jede Struktur eine Genese“ (Piaget 1966, 268, 270). In diesem Zusammenhang wird Struktur als ein System definiert, in dem einzelne Elemente zu einem Ganzen zusammengeschlossen sind, das von den Elementen unterscheidbare Eigenschaften hat und eigenen Aufbaugesetzen gehorcht. Strukturen sind keine statischen Ganzheiten, sondern Systeme von Transformationen nach bestimmten Regeln. Die Struktur wird im Allgemeinen durch Transformationen nicht zerstört, weil sie nur innerhalb der durch diese gesetzten Grenzen wirksam werden. Je mehr Umwandlungen eine Struktur zulässt, ohne dass ihr Funktionieren als Ganzes zerstört wird, umso stabiler ist sie (Piaget 1973, 7-18 in zur Oeveste 1987, 19). Um den Begriff der Struktur schärfer zu fassen, führt Piaget den Begriff des Schemas ein. „Ein Schema ist die typische Weise, eine bestimmte Klasse von Umweltgegebenheiten zu handhaben, z.B. das Schema des Werfens, das Schema der Dreisatz-Rechnung,... Die Strukturen (gemeint sind: kognitive Strukturen) in Piagets Theorie sind organische Ver- 
bindungen von Schemata. Mit einem einzigen Schema kann ein Individuum nicht viel zusammenbringen, und mit einer unverbundenen Menge von einzelnen Schemata ist nur wirre Aktivität möglich. Erst die geordnete Verbindung von verschiedenen Schemata ermöglicht einen befriedigenden Austausch mit der Welt“ (Flammer 1996, 118; [Klammer im Original] in Moser Opitz 2001, 20). Eine treffende Beschreibung der Rolle von Schemata bei der kognitiven Aktivität geben Botkin et al. (1979, 22 in Wittmann 1982, 29): „Kurz gesagt deuten neue Forschungen darauf hin, dass kognitive Prozesse im Grunde im Einpassen neuer Informationen aus der Umgebung in geeignete gedankliche Schemata bestehen. Diese sind Teil einer großen Zahl abstrakter Modelle (,frameworks"), die durch frühere kognitive Aktivitäten geschaffen wurden. Jedes Schema (,frame“) erzeugt aus den von außen eintreffenden Daten eine zusammenhängende Struktur. Unser Gehirn speichert die Gedächtnishalte nicht isoliert, sondern hält sie in vielfachen Kopien den verschiedenen Kontexten entsprechend bereit. Die Kontexte werden auf ihren Inhalt einwirken und werden ihre spätere Wiedererinnerung erleichtern. In dem Masse wie die Anzahl dieser Kontexte anwächst, werden Verstehen und Lernen tief greifend beeinflusst.“

Jean Piaget sieht das lernende, erkennende Individuum in Wechselwirkung mit der umgebenden Realität. Dieser Austausch zwischen Individuum und Umwelt wird durch die Aktivität des Individuums bestimmt. Piaget kommt zur Überzeugung, dass erst Handeln Denken überhaupt möglich macht. Die handelnde Person erweitert durch die aktive Auseinandersetzung mit der Umwelt ihr kognitives Repertoire. Aufbauend auf vererbte Strukturen am Anfang dieses Prozesses (z.B.: Saug- oder Greifreflex) spielen zwei invariante Funktionen eine wichtige Rolle zur Klärung von Entwicklungsprozessen: Anpassung und Organisation. Jeder Spezies wohnen diese zwei grundsätzlichen Tendenzen inne und sie sind zentrale Begriffe in der genetischen Epistemologie Piagets. Ginsburg und Opper beschreiben die Organisation als ,... die Tendenz bei allen Spezies, ihre Prozesse in geschlossenen Systemen zu systematisieren und zu organisieren, die entweder physisch oder psychologische sind“ (Ginsburg, Opper 1998, 33). Neben der Organisation spielt die angeborene Tendenz der Anpassung im Werk von Piaget eine übergeordnete Rolle. Alle Lebewesen werden mit der invarianten Funktion geboren, sich der Umwelt anzupassen. Die Interaktion zwischen Individuum und der umgebenden Umwelt wird durch die zwei sich ergänzenden Prozesse Assimilation und Akkommodation unterstützt, beziehungsweise überhaupt in Gang gehalten (Moser Opitz 2001, 20). Piaget definiert Assimilation als „Tendenz, Verhaltensweisen wieder zu vollziehen und sie an 
immer neuen Gegenständen ablaufen zu lassen“ (Piaget 1975a, 52). Dieses Verhalten muss als das Streben angesehen werden, Informationen aus der Umwelt in verfügbare kognitive Schemata einzufügen; Ereignisse aus der Umwelt für die jeweilige Erkenntnisstruktur passend zu machen. Mit Akkommodation bezeichnet Piaget die komplementäre Tendenz, individuelle Strukturen gemäß den Anforderungen und objektiven Gegebenheiten der Umwelt zu verändern. Dieser Prozess kann in einer Veränderung des Verhaltens, aber auch einer Umformung begrifflicher Schemata bestehen (zur Oeveste 1987, 20).

Die beiden Prozesse der Assimilation und der Akkommodation laufen im Prinzip immer gleichzeitig ab und haben eine komplementäre Wirkung. Das führt dazu, dass sich Schemata und kognitive Strukturen im Laufe der Zeit verändern. Diese Veränderung vollzieht sich nicht unsystematisch, sondern die Strukturen bilden eine Hierarchie. Diese Erkenntnis führt zu der für die Theorie von Piaget so wichtigen Definition der Entwicklungsstufen.

„Im Laufe des Lebens bleiben die Funktionen gleich, die Struktur verändert sich jedoch in einem nahezu regelmäßigen Rhythmus. Mit andern Worten besteht die intellektuelle Entwicklung aus einer Folge von Stadien. Jedes Stadium wird durch eine andere Art psychologischer Strukturen gekennzeichnet“ (Ginsburg; Opper 1998, 39).

Um die Frage nach der Entwicklung zu klären, hat Piaget den Begriff der Äquilibration eingeführt. Viele kognitive Konflikte, Probleme und Widersprüche, die im Laufe des Heranwachsens auftreten, können mit den vorhandenen Schemata nicht gelöst werden. Das Streben, einen entstandenen Ungleichgewichtszustand wieder in einen Gleichgewichtszustand überzuführen, treibt die Entwicklung voran (vgl. Moser Opitz 2001, 21). „Es wird ein Äquilibrium, ein Gleichgewichtszustand erreicht, wenn Assimilation und Akkommodation ausgeglichen sind. Alles Verhalten steht im Dienst der Sicherung eines Äquilibriums. Dieses Verhalten wird jedoch nicht ein für alle Mal erreicht, sondern es ist das Ergebnis einer dauernden Interaktion von Assimilation und Akkommodation. Es ist ein dynamischer Lebensprozess im Sinne eines Entwicklungskontinuums zwischen fundamentalen Austauschprozessen (Assimilation/Akkommodation) bis $\mathrm{zu}$ höchsten kognitiven Leistungen eines Erwachsenen, in denen diese basalen Prozesse einerseits aufgehoben sind, sich andererseits dynamisch weiterentwickeln“ (Bundschuh 1995, 120 in Moser Opitz 2001, 21). 


\subsection{Entwicklung und Lernen nach Piaget}

Zur Entwicklung gehört also ein Widerspruch zwischen vorhandenen Schemata, die Assimilation neuer Probleme an diese Schemata, sowie eine sich selber regulierende Angleichung oder Weiterentwicklung der aktuellen Denkweisen (Ginsburg; Opper 1998, 272). Das aktive und handelnde Individuum, das sich mit der Umwelt auseinandersetzt, steht im Zentrum von Piagets Verständnis von Entwicklung und Lernen. Die Aktivität und die Initiative des Kindes spielen in der Entwicklung die zentrale Rolle. Demzufolge ist aus der Sicht von Piaget Lernen nicht ein Prozess, der durch Vermittlung bestimmt wird, sondern Ergebnis von Entwicklung (Moser Opitz 2001, 21). Vom pädagogischen Standpunkt aus bedeutet das, dass es nicht Aufgabe des Unterrichts ist, den Kindern etwas beizubringen, sondern sie zum Mittun und zum Selbermachen anzuregen. Das Kind soll aktiviert, nicht konditioniert oder programmiert werden (Kesselring 1999, 65). Es kommt darauf an, „dass der Schüler jeden Tatbestand, den er sich aneignen soll, von sich aus neu findet oder doch zumindest nachvollzieht und nicht einfach nur übernimmt“" (Piaget 1975, 78). Die kognitive Aktivität lässt das Individuum die eigenen Strukturen verändern. Es entwickelt sich und lernt. Die Entwicklung in diesem Sinne enthält nach Piaget vier Faktoren: Reifung; Erfahrung und Umgang mit Gegenstände; soziale Vermittlung und Äquilibrationstendenz (Ginsburg, Opper 1998, 272ff.).

Reifung: Die geistige Entwicklung wird nach Piaget von verschiedenen, dem Kind angeborenen organischen Strukturen beeinflusst. Bei diesen angeborenen, artspezifischen physiologischen Strukturen handelt es sich um Erkenntnisorgane, die den groben Umriss geistigen Wachstums abstecken und die sich mit zunehmendem Alter in stetiger Auseinandersetzung mit der Umwelt zu Funktionen höherer Ordnung entwickeln. „Ganz allgemein wird durch die Reifung des Gehirns und des zentralen Nervensystems das Kind in die Lage versetzt, zu denken und Sprache zu erwerben. Für Piaget lautet die Frage nicht, ob die Reifung eine Wirkung zeitigt, sondern wie groß die Bedeutung der Reifung ist und wie sie funktioniert" (Ginsburg, Opper 1998, 272ff.).

Erfahrung und Umgang mit Gegenständen: Wie schon mehrfach erwähnt, macht für Piaget erst Handeln Denken möglich. Durch den Umgang und die Auseinandersetzung mit Gegenständen macht das Kind physikalische Erfahrungen. Durch die geistige Auseinandersetzung und die dadurch nötige Anpassung vorgegebener Strukturen wandelt sich diese physikalischen Erfahrungen in übergeordnete logische Erfahrungen (Moser Opitz 2001, 22). 
Soziale Vermittlung: Die in der Einleitung erwähnten Interaktionen der Kinder mit Eltern, Geschwistern sowie Spielkameraden sind dazu geeignet, Denkprozesse auszulösen. Andere Personen initiieren Aktivitäten (,Zähl mal diese Äpfel.“), die dann neue Schemata schaffen; ein Lernprozess kommt in Gange. Das heranwachsende Kind kann aber nur solche Informationen aufnehmen, die seinem kognitiven Entwicklungsstand entsprechen. Ist das der Fall, können sich Lernprozesse fruchtbar auswirken (zur Oeveste 1987, 25).

Äquilibration: Die obigen drei Faktoren vermögen keine für sich die geistige Entwicklung hinreichend zu erklären. Die Äquilibration integriert in einem gewissen Sinne die Auswirkungen dieser drei Faktoren; dabei handelt es sich um „die sich selbst regulierenden Prozesse des Kindes, aufgrund derer es von Entwicklungsstadium zu Entwicklungsstadium zunehmend höhere und bessere Formen des Gleichgewichtszustandes erreicht. Der Äquilibrationsprozeß ist die Grundlage des geistigen Wachstums“"(Ginsburg, Opper 1998, 283 in Moser Opitz 2001, 22).

Für Piaget setzen Lernprozesse die Äquilibration voraus, während umgekehrt Äquilibrationsprozesse nicht erlernbar sind (Kesselring 1999, 180). Die geistige Aktivität des Kindes ist es, die Lernen ausmacht und dazu führt, dass das kognitive Repertoire sich immer weiter entwickelt. Obwohl Piaget kein praktizierender Pädagoge war, hat er sich verschiedentlich dazu geäußert. Nicht Wissen und Inhalte müsse die Lehrperson weitergeben, sondern Werkzeuge, die dem Kind Begreifen und Lernen ermöglichen. Außenstehende Personen können somit Lernen allenfalls arrangieren und begünstigen, nicht aber garantieren. „Das Kind ist aber kein passives Wesen, dessen Hirn es voll zu stopfen gilt, sondern ein aktives Wesen, das in seiner spontanen Suche nach Wissen gefördert werden will. ... Jetzt geht es darum, dass Fragen, die sich die Schüler selber stellen, beantwortet werden“ (Piaget 1999, 182). Oft stellen die Kinder aber nicht die richtigen Fragen, darum sei es die Aufgabe der Lehrperson, „Anstoß und Anregung zu geben, d.h. zunächst die Situationen herbeizuführen und die Grundvoraussetzungen zu schaffen, die das Kind vor nützliche Probleme stellen, um es anschließend durch geeignete Gegenbeispiele zum Nachdenken und damit zur Überprüfung vorschneller Lösungen zu nötigen“ (Piaget 1975, 78). 


\subsection{Entwicklungsstadien und Zahlbegriff}

Der Erwerb des Zahlbegriffs ist nach der Auffassung von Piaget eingebettet in der allgemeinen kognitiven Entwicklung. So wie alle mathematischen Strukturen eine Konstruktion seien, so ist auch der Erwerb des Zahlbegriffs konstruiert aus der Erfahrung mit mathematischen Gesetzmäßigkeiten, die sich aus den konkreten Operationen ergeben. „Dadurch wird nun verständlich, warum die Konstruktion der logischmathematischen Strukturen streng genommen weder Erfindung noch Entdeckung ist: da sie sich vermittels reflektierender Abstraktionen vollzieht, ist sie Konstruktion im engeren Sinn, das heißt, sie bringt neue Kombinationen hervor" (Piaget 1992, 328). Diese Konstruktion im Bezug auf den Erwerb des Zahlbegriffs stützt sich insbesondere auf Erfahrungen mit der Seriation ${ }^{5}$ und der Klassifikation ${ }^{6}$ von Gegenständen. (Im Übrigen beantwortet hier Piaget auch die Frage nach dem Ursprung der Zahlen: Die Zahl ist für Piaget eine Konstruktion; keine Erfindung und keine Entdeckung.)

Es scheint mir nützlich, die Entwicklung des jungen Menschen kurz darzustellen und in diesem Kontext die Zahlbegriffsentwicklung einzubetten. Ich halte mich bei dieser Darstellung an die Entwicklungspsychologie von Jean Piaget, die in Moser Opitz (2001) sehr umfassend dargestellt wird. Die kognitive Entwicklung von der Geburt bis zur Adoleszenz hat Piaget in vier Stufen unterteilt. Piaget hat Kriterien angegeben, die zur Unterscheidung dieser Stufen dienen. Danach soll gelten, „dass sie bei allen Versuchspersonen in einer konstanten Reihenfolge aufeinanderfolgen, dass jede durch eine Gesamtstruktur charakterisiert werden kann (und nicht nur durch eine dominierende Eigenschaft) und dass sich diese Strukturen gemäß ihrer Entwicklungsfolge einander wechselseitig integrieren“ (Piaget; Inhelder 1979, 466). Schemata tieferer Stufen werden demzufolge in höhere Stufen übernommen und in diese eingebaut. Fähigkeiten gehen so nicht verloren, sondern bleiben in veränderter Form in den höheren Stufen erhalten. Da vor allem die ersten zwei, die präoperationale und die konkret-operationale Stufe für die Entwicklung des Zahlbegriffs für uns von Bedeutung sind, seien sie hier breiter vorgestellt. Die übrigen Stufen sind nur der Vollständigkeit halber kurz beschrieben. Die von Piaget angegebenen Altersangaben dürfen nicht zu streng gesehen werden, sondern dienen als Richtzeiten.

\footnotetext{
${ }^{5}$ Gegenstände unterschiedlicher Größe in eine Reihenfolge bringen, z.B. nach Größe, Tonhöhe, Länge.

${ }^{6}$ Ordnen von Gegenständen nach einem Merkmal, z.B. nach Größe, Farbe, Form, Gewicht.
} 
Von der Geburt bis etwa zum 2. Lebensjahr entwickelt sich beim Kind so vieles in so kurzer Zeit wie später nie wieder (Kesselring 1999, 102). Piaget gliedert die ersten zwei Lebensjahre in sechs verschiedene Stadien, die er in einigen Darstellungen zu drei Perioden zusammengezogen hat:

1. Reflexe als ererbte Reaktionen

1. Monat

2. Erste motorische Gewohnheiten

2. - 4. Monat

3. Sensomotorische (praktische) Intelligenz

5. - 24. Monat

(zur Oeveste 1987, 20)

Piaget nennt diese ersten drei Perioden zusammenfassend sensomotorisch, ,... weil das Kleinkind, mangels einer symbolischen Funktion, noch kein Denken und keine Affektivität zeigt, die mit Vorstellungen verbunden wären, durch die es Personen oder Gegenstände in ihrer Abwesenheit bezeichnen könnte“ (Piaget; Inhelder 1981, 11). Wie der Name dieser Stufe aussagt, sind die zentralen Funktionen dieser Stufe Wahrnehmung (Senso-) und Bewegung (Motorik). Durch diese psychologische Struktur werden Reflexe und einfache Reiz-Reaktionsschemata durch die oben beschriebenen Prozesse zu Frühformen des Denkens entwickelt (Moser Opitz 2001, 24). Gekennzeichnet ist diese Periode dann auch durch eine umfangreiche Entwicklung und wird vielfach unterschätzt, da die Fortschritte der Intelligenz noch nicht an den verbalen Äußerungen gemessen werden können. Die Babys erkunden ihre Umwelt mit ihren fünf Sinnen und lernen, sie durch ihre motorische Aktivität zu steuern. Durch verbesserte Koordination von Bewegungen und Wahrnehmungen bemächtigt sich das Kind nach und nach der gesamten praktischen Umwelt (zur Oeveste 1987, 21). Genauso wie jede andere abstrakte Repräsentation der Welt, so glaubte Piaget, müsse auch der Zahlbegriff durch sensomotorische Wechselwirkung mit der Umwelt konstruiert werden. Die Kinder erfassen nach dieser Auffassung erst nach Jahren intensiver Beobachtung was eine Anzahl ist. Erst durch den Umgang mit Objekten erfahren die Kinder allmählich, dass die Anzahl die einzige Eigenschaft ist, die sich nicht verändert, wenn sich die Lage oder das Aussehen der gezählten Dinge verändert. So beschreibt Seymour Papert diesen Vorgang:

„Für den Säugling gibt es noch gar keine Objekte. Es braucht eine anfängliche Strukturierung, damit sich Erfahrung zu Objekten ordnet. Wir betonen ausdrücklich, dass das Baby die Existenz von Objekten nicht entdeckt, wie ein Naturforscher einen Berg entdeckt, sondern eher so, wie jemand die Musik entdeckt. Vielleicht hat er sie schon seit Jahren gehört, aber sie war für seine Ohren lediglich ein Geräusch. Wenn das Kind Objekte erworben hat, muss es noch einen weiten Weg zurücklegen, bevor es die Stufe der 
Klassen, der Anordnung, des Enthaltenseines und schließlich der Zahl erreicht“ (Papert 1960 in Dehaene 1999, 55). Wie wir ab den Seiten 19 zu zeigen versucht haben, deckt sich diese Einschätzung nicht mehr mit den heutigen Auffassungen. Kinder haben sehr wohl eine Meinung, was Quantitäten angeht. Die Kinder verfügen in dieser Phase schon über einen Zahlbegriff.

Die darauf folgende präoperationale Stufe ist für die vorliegende Arbeit von zentraler Bedeutung. Nach Piaget erstreckt sich diese Stufe auf die Alterspanne zwischen zwei und sieben Jahren und ist in zwei Phasen unterteilt, die symbolisch-vorbegriffliche Phase (2 - 4 Jahre), sowie die Phase des anschaulich-intuitiven Denkens (4 - 7 Jahre).

Die symbolisch-vorbegriffliche Phase: Mit dem Erwerb der Symbolfunktion und der Sprache beginnt das Stadium der präoperationalen Stufe. Die Elementarform der Symbolfunktion ist die Fähigkeit, Handlungen und Gegenstände, die nicht gegenwärtig sind, durch Zeichen oder eben Symbole zu ersetzen. Während in der ersten Stufe Handeln und Wissen eins sind, treten sie im Übergang zur zweiten auseinander: Das Kind wird fähig, sich reflexiv auf seine eigenen Handlungen zu beziehen, sie sich vorzustellen (Kesselring 1999, 113). Das sensomotorische Kind stellt sich die Dinge dadurch vor, dass es die Bewegungen ausführt. Nach der Ansicht von Piaget gelangt das Kind von einer innerlichen, kaum wahrnehmbaren Nachahmung der physischen Bewegung allmählich zum definitiven Vorstellungsbild. „Das ältere Kind verinnerlicht diese Nachahmung. Diese abgekürzten Körperbewegungen konstituieren die symbolische Vorstellung. Schließlich beherrscht es die innere Nachahmung so gut, dass es die Bewegungen bis zur Unmerklichkeit abzukürzen vermag“ (Ginsburg, Opper 1998, 100). Durch die Symbolfunktion wird das Kind in die Lage versetzt, sich innere Bilder von Handlungen und Gegenständen zu bilden. Diese Handlungen und Dinge können nach und nach benannt werden.

Das Symbolspiel bestimmt die Aktivitäten des Kindes in diesem Alter in einem ganz beträchtlichen Maße. Mit dieser Interaktionsform kann das Kind die Außenwelt fast vollständig seinen Wünschen assimilieren, statt diese der Welt akkommodieren zu müssen (Ginsburg, Opper 1998, 100). Das Kind sieht sich in der Lage, eine Nebenwelt nach eigenen Bedürfnissen zu schaffen. Lange bevor Kinder zählen können, sind sie in der symbolisch-vorbegriffliche Phase in der Lage Quantitäten zu erkennen. K. Wynn (1990, 1992a) beschreibt, dass Kinder wissen, dass sich Zahlwörter auf Quantitäten beziehen und so über einen schon differenzierten Zahlbegriff verfügen. Das noch während des Spracherwerbs; nämlich schon mit 2⿺辶⿸厃㔾 Jahren. Sie können „eins“ und „viele“ und andere 
Zahlwörter voneinander unterscheiden. Frappante Untersuchungsergebnisse von Carey (1982) und Soja, Carey und Spelke (1991) zeigen, dass Kinder aus dem sprachlichen Kontext erkennen, wenn es sich um noch unbekannte Zahlwörter oder andere unbekannte Eigenschaftswörter handelt. Die Aufforderung:,,Gib mir den chromium Bauklotz, nicht den grünen“ lässt Kinder aus der Kontrastierung der Begriffe erkennen, dass es sich bei „chromium“ um ein Farbwort handelt. Kinder erfassen offenbar sehr schnell, dass im Gegensatz zu andern Eigenschaftswörtern bei Mengenangaben kein bestimmter oder unbestimmter Artikel vorangeht: „Gib mir drei Bauklötze“ im Gegensatz zu „Gib mir die roten Bauklötze“. K. Wynn (1992a) konnte zeigen, dass gerade solche Informationen von Kindern herangezogen werden, um sich in der Kommunikation mit anderen Menschen zurechtzufinden (vgl. Stern 1998, 58).

Das anschaulich-intuitive Denken: Das anschauliche Denken entwickelt sich im Vorschulalter und stellt eine Übergangsphase zwischen dem symbolisch-vorbegrifflichen und operatorischen Denken dar. Das Kind ist jetzt befähigt, Schlussfolgerungen (WennDann-Verbindungen) zu bilden. Nach Piaget unterliegt das präoperationale Denken aber einer anschaulichen Zentrierung und fehlender Reversibilität. Reversibilität meint die Fähigkeit, „einen Vorgang im Geist zurückzuverfolgen und zum Ausgangspunkt zu gelangen, um ihn mit dem gegenwärtigen Zustand zu vergleichen“ (Stendler-Lavatelli 1976, 39). Das Denken der Vorschulkinder ist noch stark auf den eigenen Standpunkt und die eigene Perspektive bezogen (Egozentrismus) und weist zudem einen Mangel an Sozialisierung und Allgemeinheit auf. Dem Kind fällt es schwer, Transformationen als Handlungsfolgen wahrzunehmen, es neigt dazu, nur einzelne statische Situationen zu betrachten. Das Kind ist nach Piaget noch nicht fähig, seine Aufmerksamkeit gleichzeitig auf zwei oder drei Aspekte zu richten und diese miteinander zu koordinieren. Somit kann das Kind noch nicht Zustände und Transformationen miteinander in Beziehung bringen. Durch den Egozentrismus richtet es die Aufmerksamkeit auf das eine oder das andere, nicht auf beide zusammen. So falle es den Kindern schwer, die kommunikative Funktion von Gesprächen wahrzunehmen und sich auf den Standpunkt eines Partners einzustellen (zur Oeveste 1987, 21). Prä-operationales Denken ist egozentrisches Denken. Infolge dessen wird es für die Kinder kaum möglich, Ganzes und Teile in Beziehung zu setzen, oft ist die Aufmerksamkeit nur auf das eine gerichtet und das andere ist „aus dem Sinn“. Das Kind orientiert sich an seiner sinnlichen Wahrnehmung; daran, was es sieht, hört, riecht oder spürt und diese Wahrnehmung steht im Zentrum des Tuns und Denkens (Stendler-Lavatelli 1976, 38 in Moser Opitz 2001, 25). Margaret Donaldson 
(1982) aber legt zahlreiche Belege vor, die zeigen, dass diese Vorgabe des Egozentrismus fallengelassen werden muss. Kinder im Vorschulalter sind auch bessere Kommunikationspartner als allgemein angenommen. Voraussetzung ist allerdings, dass der erwachsene Gesprächsteilnehmer seinen Standpunkt aufgeben kann und sich bemüht, die Sicht des Kindes als gleichwertig anzunehmen (Donaldson 1982, 20-36).

Für die Genfer Schule um J. Piaget ist das Invarianzprinzip (oder Erhaltungsprinzip) von zentraler Bedeutung. So bedeutend, „dass die Invarianz eine notwendige Bedingung jeder verstandesmäßigen Tätigkeit darstellt“ (Piaget, Szeminska 1975b, 15). Für den Erwerb des Zahlbegriffs ist die Invarianz eine wesentliche Voraussetzung; denn „eine Menge oder eine Gruppe von Gegenständen ist nur vorstellbar, wenn ihr Gesamtwert unverändert bleibt, gleich welche Veränderungen in den Verhältnissen der Elemente eintreten mögen. [...] Eine Zahl ist nur in dem Maße verständlich, wenn sie mit sich selber gleich bleibt, unabhängig von der Disposition der Einheiten, aus denen sie zusammengesetzt ist: Das ist die sogenannte ,Invarianz’ des Zahlbegriffs“ (Piaget; Szeminska 1975b, 16; Anführungszeichen im Original). Die Kinder erwerben nach J. Piaget das Invarianzprinzip (oder Erhaltungsprinzip) in einer Übergangsphase $(5 ; 6-7 ; 6 \mathrm{~J} .)^{7}$. Um diese Invarianz zu überprüfen wird ,die in der kognitiven Entwicklungspsychologie am häufigsten eingesetzte Aufgabe“ (Stern 1998, 62) wie folgt durchgeführt: Eine Anzahl von Objekten (z.B. Knöpfe, roten Bätzeli oder Holzwürfelchen) werden in eine Reihe gelegt. Unterhalb dieser Reihe von roten Bätzeli wird eine Reihe von blauen Bätzeli angebracht, so dass unter jedem roten Bätzeli ein blaues zu liegen kommt. Bei dieser eineindeutigen Zuordnung von Bätzeli bestätigen alle drei- bis vierjährigen Kinder, dass es in beiden Reihen gleich viele Bätzeli hat. Anschließend wird eine der Reihen, z.B. die mit den roten Bätzeli, vom Versuchsleiter auseinandergezogen. Die Abstände zwischen den roten Bätzeli wird so größer und die Reihe länger. Die Kinder werden gefragt: „Sind in beiden Reihen gleichviel Bätzeli, oder sind in einer Reihe mehr Bätzeli?“" Kinder unter sechs Jahren werden antworten, dass es mehr rote Bätzeli gibt. Entsprechende Ergebnisse zeigen sich ebenfalls, wenn eine Reihe verkürzt wurde, indem die Bätzeli zusammengeschoben wurden. In der Theorie Piagets signalisiert das Scheitern bei der Zahlinvarianzaufgabe die für die präoperationale Stufe charakteristischen Defizite: eine einmal vollzogene Handlung kann in der Wissensrepräsentation nicht rückgängig gemacht werden.

\footnotetext{
${ }^{7} \mathrm{n}$;m bedeutet $\mathrm{n}$ Jahre und $\mathrm{m}$ Monate
} 
Siegler (1981) sowie Cowan (1979) konnten zeigen, dass bereits vierjährige Kinder die Zahlinvarianzaufgabe richtig lösten, wenn eine Reihe aus bis zu vier Objekten bestand. Dies kann mit der Fähigkeit zur Mengenschätzung erklärt werden: Kinder, die noch nicht wissen, dass man die beiden Reihen durch Zählen oder durch eine Eins-zu-einsZuordnung vergleichen kann, können bei längeren Reihen nicht herausfinden, dass beide Reihen die gleiche Anzahl von Bätzeli enthalten. Bei Reihen mit bis zu vier Objekten hingegen sehen sie simultan, dass beide Reihen gleich viele Objekte enthalten. Nach Klahr (1984) und Klahr und Wallace (1976) entsteht das Invarianzprinzip durch die Generalisierung der bei der Mengenschätzung erworbenen Prinzipien (Stern 1998, 63). Mehler und Bever aber zeigten 1997, dass es ratsam ist, vorsichtig bei der Interpretation solcher Versuche zu sein. In einer der beiden Versuchsreihen legten sie entsprechen der oben beschriebenen Versuchsanordnung zwei Reihen mit Murmeln aus. Eine Reihe war kürzer und bestand aus sechs Murmeln. Die andere Reihe war zwar länger, hatte aber nur vier Murmeln. Bei der üblichen Frage, welche der beiden Reihen mehr Murmeln enthalte, gaben die meisten der zwischen zwei und vier Jahre alten Kinder die falsche Antwort und wählten die längere Reihe, obwohl sie aus weniger Murmeln bestand. In der zweiten Versuchsreihe ersetzten Mehler und Bever die Murmeln mit Bonbons und stellten den Kindern keine Fragen, sondern ließen sie eine der beiden Reihen aussuchen und die Bonbons gleich essen. Bei dieser Versuchanordnung gab es keine Verständigungsdifferenzen zwischen Versuchsleiter und Kinder. Die meisten Kinder wählten nun die Reihe mit den meisten Bonbons auch dann, wenn sie nicht die längere Reihe bildeten.

In den oben beschriebenen Experimenten von Mehler und Bever bewältigten zweijährige Kinder den Test sowohl mit Murmeln als auch mit Bonbons. Erst ältere Kinder bemerkten nicht, dass bei Verlängerung der Reihe die Anzahl der Murmeln gleich geblieben ist. Es scheint so, als ob etwas ältere Kinder die Mengeninvarianz vorübergehend weniger gut erfassen würden. Sicher aber sind die kognitiven Fähigkeiten drei- bis vierjähriger Kinder den zweijährigen Kindern überlegen. Offenbar überprüfen Versuche von Piaget nicht wirklich die numerischen Kompetenzen von Kindern. Es scheint, dass diese Versuche ältere Kinder so sehr verwirren, dass sie nicht annähernd so gute Resultate erzielten wie jüngere Kinder (vgl. Dehaene 1999, 57ff). Es ist anzunehmen, dass dreibis vierjährige Kinder im Gegensatz zu zweijährigen Kindern die Frage nach der Quantität der beiden Reihen im ursprünglichen Test von Piaget anders verstehen als Erwachsene. Die Testanordnung ist so angelegt, dass man die Frage nach der Anzahl Elemente 
in den beiden Reihen auch uminterpretieren könnte nach der Länge der Reihe. Auch drei- bis vierjährige Kinder finden es offenbar merkwürdig, eine derart triviale Frage nach der Anzahl Elemente zweimal zu stellen. Die Antwort ist ja offensichtlich und verletzt zudem die üblichen Konventionen der Gesprächsführung. Es ist unüblich, eine Frage zu stellen, deren Antwort beide Gesprächspartner schon kennen. Das einzige, was sich gegenüber früher verändert hat, ist die Länge der Reihe. Deshalb muss für die Kinder die Frage wohl etwas mit der Länge zu tun haben.

Ungefähr mit Beginn des 7. Lebensjahres entwickelt sich die Stufe der konkreten Operationen des Denkens. Dieser Lebensabschnitt wird nach Piaget mit dem 11./12. Lebensjahr abgeschlossen. Diese Stufe ist für die Darstellung und den Aufbau des Zahlbegriffs von großer Bedeutung. Piaget definiert gerade diese konkreten Operationen als Voraussetzung für den Erwerb des Zahlbegriffs.

Konkret werden die geistigen Operationen darum genannt, weil sie sich ausschließlich auf konkrete Informationen über Objekte im Wahrnehmungsfeld der sieben- bis elfjährigen Kinder beziehen. Die Kinder können ihre Denkvorgänge noch nicht mittels verbal formulierter Hypothesen vollziehen (Piaget, Inhelder 1981, 76). Schröder (1989, 20 in Moser Opitz 2001, 26) gibt vier Merkmale an, welche das konkret-operatorische Denken vom Denken der präoperatorischen Stufe abgrenzen:

- Zentrierung versus Dezentrierung: Die Dezentrierung gewinnt gegenüber der Zentrierung die Oberhand. Mehrere Aspekte einer Situation oder Handlungen können vom Kind wahrgenommen und bei Bedarf koordiniert werden. Das Kind gewinnt Übersicht. Einzelne Schritte und Ziele in einem Handlungsablauf können im Blick behalten werden.

- Wahrgenommene Wirklichkeit versus erschlossene Wirklichkeit: Das Kind lässt sich nicht mehr durch seine Wahrnehmung täuschen. Das Kind wird unabhängiger von seiner Wahrnehmung und kann Zusammenhänge auch innerlich erschließen.

- Zustände versus Transformation: Handlungsabläufe werden als Einheit wahrgenommen. Die einzelnen Schritte werden als Ganzes aufgefasst und zueinander in Beziehung gebracht. Statische Momentaufnahmen kommen immer seltener vor.

- Irreversibilität versus Reversibilität: Das Kind kann vom Endzustand einer Handlung auf deren Anfangszustand schließen. 
Der Zahlbegriff mit allen erwähnten Aspekten (Kardinal-, Ordinal-, Maßzahl-, Operator, Rechenzahl- und Codierungsaspekt) ist mit elf Jahren bei den allermeisten Jugendlichen umfassend vorhanden.

Die folgende Stufe der formalen Operationen sei nur der Vollständigkeit halber aufgeführt. Operationen mit reinen Aussagen, die bloß hypothetischen Charakter haben, entwickeln sich erst nach dem 11. Lebensjahr. Es ist die Phase, „, in der sich der junge Mensch vom Konkreten löst und das Wirkliche in ein System von möglichen Transformationen einordnet. Diese grundlegende Dezentrierung vollzieht sich am Ende der Kindheit und bereitet die Adoleszenz vor, deren wichtigstes Kennzeichen zweifellos eine solche Lösung vom Konkreten und eine Hinwendung zu solchen Interessen ist, die sich auf das Unaktuelle und die Zukunft richten: das Alter der großen Ideale und der einsetzenden Theorien über die schon vorhandenen einfachen Anpassungen an das Wirkliche hinaus“ (Piaget, Inhelder 1981, 97). 


\section{Zählkompetenz}

\subsection{Die Bedeutung des Zählens}

„Die Zahlenreihe ist die Grundlage der Mathematik - historisch, genetisch und systematisch. Ohne die Zahlenreihe gibt es keine Mathematik - wenn es nach manchen modernen Mathematikbüchern anders scheinen mag, so kommt das daher, dass ihre Verfasser nicht wissen, was Mathematik ist. Das kleine Kind weiß es besser. Ihm wird das Zählen schnell zum theoretischen Bedürfnis“ (Freudenthal 1973, 160).

Diese dezidierte Aussage muss als Gegengewicht zu der Gestaltung und Strukturierung eines Erstrechenunterricht angesehen werden, wie er auch heute noch vielfach durchgeführt wird: Nach einem ausführlichen pränumerischen Teil folgt oft eine Einführung der natürlichen Zahlen auf der Basis des Kardinalzahlbegriffs. Die Vorkenntnisse der Zahlen und die Zählkompetenz werden oft gar nicht wahrgenommen und auch heute noch häufig unterschätzt (vgl. z.B. Hengartner/Röthlisberger 1994). Die Betonung auf einsichtsvolles Lernen sowie große Gewichtung mathematischer Prinzipien und Strukturen mögen die Gründe dafür sein, den Zugang zum Zahlbegriff über den Kardinalzahlaspekt zu wählen. Dem konstruktiven Aufbau der Zahlstruktur sollte ein verbaler Umgang mit diesen Strukturen vorangehen (Schmidt/Weiser 1982, 227). Weiter verantwortlich für diesen Weg resp. die Unterschätzung des Zählens sind z.B. das folgende KardinalzahlArgument: „Zahlbegriff und Zählen sind zwei völlig verschiedene Dinge. Diese Erkenntnis der modernen Mathematik ist grundlegend. Nachdem die Gleichzahligkeit zweier oder mehrerer Mengen betrachtet wurde, kann der Begriff Zahl selbst in den Blick genommen werden“ (Schlechtweg/Buchmann 1970, 75). Piaget selbst hat für den Aufbau eines Zahlbegriffs das Zählen als bedeutungslos betrachtet. Für ihn war Zählen ein Daherplappern eines auswendig gelernten Sprüchleins, ein Reproduzieren von memorierten Worthülsen ohne numerische Bedeutung (vgl. Moser Opitz 2001, 63). „Die gesprochene Aufzählung, die die soziale Umgebung dem Kinde dieses Niveaus (Stadium 1, präoperational; Einfügung F.C.) zuweilen aufzwingt, bleibt in der Tat völlig verbal und ohne operatorische Bedeutung“ (Piaget, Szeminska 1975b, 47). Im Weiteren ist das Mengeninvarianz-Argument für diesen oben geschilderten Erstrechenunterricht verantwortlich: „Auch die von Eltern im Vorschulalter ihrer Kinder oft gepflegte Fähigkeit des Zählens garantiert keineswegs das Erreichen der Stufe der Mengeninvarianz. [...], dass die Fähigkeit zum Zählen noch kein weiteres Indiz für ein Grundverständnis des 
Anzahlbegriffs ist. [...] Bemerkenswert ist [...] die Tatsache, dass selbst Kinder, die sich sprachlich richtig im Sinne des quantifizierenden Zählens äußern, noch nicht auf der Stufe der Mengeninvarianz zu sein brauchen, [...] Man wird hier von einer scheinbaren Quantifizierung sprechen können“ (Schwirtz 1976, 102 in Schmidt/Weiser 1982, 227228).

Demgegenüber verweise ich auf ein Zitat von H. Freudenthal: „Ich sehe viel mehr Möglichkeiten für eine Begründung der natürlichen Zahlen und des Rechnens mit ihnen als Zählzahl, von der vollständigen Induktion her, wobei ich noch nicht an Peanosche Axiome oder auch nur eine explizite Formulierung der vollständigen Induktion denke. Das ist eine ehrliche, saubere, beispielhafte und folgenreiche Methode; solch eine Begründung der natürlichen Zahl als Zählzahl ist eine Möglichkeit, aber das bedeutet nicht: eine Notwendigkeit. Das ist - kann man jetzt wohl sagen - erwiesenermaßen eine Unmöglichkeit“ (Freudenthal 1973, 174).

Die Mengeninvarianz gilt nach den Arbeiten von Piaget als Voraussetzung für das Verstehen des Anzahlbegriffs sowie für das Betreiben von Arithmetik der natürlichen Zahlen. Es kann nichts eingewendet werden, das Verfügen der Mengeninvarianz als Bestandteil eines fundier-ten Begriffs der natürlichen Zahlen zu sehen. Ebenso wenig garantiert selbst eine elaborierte Zählkompetenz das Verfügen über die Mengeninvarianz. Arbeiten von Saxe (1979) zeigen, dass “die Elaborierung der Zählkompetenz [...] überwiegend dem Verfügen über die Men-geninvarianz voraus[geht]“ (Schmidt/Weiser 1982, 229). Die Befunde lassen aber nicht zu, die Zählkompetenz als Grundlage für die Entdeckung der Mengeninvarianz zu sehen. Saxe zeigt, dass es Kinder gibt, die unsicher und ungenau beim Zählen sind und dennoch über die Mengeninvarianz verfügen (Saxe 1979, 186). Viele Autoren haben gezeigt, dass Kinder auch ohne Mengeninvarianz durchaus in der Lage sein können, auf differenziert ausgebildetem Niveau arithmetische Probleme zu lösen (Mpiangu/Gentile 1975; Ginsburg 1977; Pennington 1977; Baroody 1979). „Auch ohne das Erreichen des Stadiums der Mengeninvarianz [...] kann ein ,Stück Arithmetik der natürlichen Zahlen' vom Kind , erobert' werden, ohne dass man dies als sinnentleert und nicht fortsetzbar abtun kann“ (Schmidt/Weiser 1982, 230).

Wie schon erwähnt, müssen für eine umfassende Zahlbegriffsbildung ebenso wie für einen erschöpfenden Arithmetikunterricht alle Aspekte der natürlichen Zahlen einbezogen werden. Darüber besteht in der fachdidaktischen Diskussion schon seit einigen Jahren Konsens. „Im Unterricht sind diese Aspekte als verschiedene Ansätze zu betrachten, die im unterrichtlichen Geschehen zur Zahlauffassung des Schülers konvergieren müs- 
sen. [...] Im Vorwissen sind die einzelnen Aspekte nicht sauber trennbar, sie können auch im Anfangsunterricht nicht isoliert werden“ (Glatfeld 1976, 142). In einem für die Kinder wie für die Lehrkraft stressreduzierten und erfolgreichen Erstunterricht in Mathematik sollte darum zur Kenntnis genommen werden, ,[...] dass das Zählen für die Kinder der wichtigste Zugang zu allen genannten Aspekten ist, vor allem zum kardinalen und ordinalen Aspekt sowie zum Skalenaspekt" (Schmidt/Weiser 1982, 231). Viele weitere Untersuchungen bestätigen diese Auffassung, dass dem Zählen für die Zahlbegriffsentwicklung sowie für den Anfangsunterricht große Bedeutung beigemessen werden muss (vgl. z.B. Gelman/Gallistel 1978; Fuson 1982; Brainerd 1979; Comiti 1980; Fischer 1982). „Das Zählen ist das Schweizer Taschenmesser des Rechnens, ein Werkzeug, das Kinder spontan für alle möglichen Zwecke nutzen. Mit Hilfe des Zählens finden fast alle Kinder ohne explizite Unterweisung Möglichkeiten, Zahlen zu addieren und zu subtrahieren“ (Dehaene 1999, 143). Die Auffassung, dass Zählen zwar nicht die einzige, doch eine wichtige und zentrale Voraussetzung zur Erlangung numerischer Kompetenzen ist, hat sich mittlerweile in vielen Schulbüchern niedergeschlagen (z.B. Zahlenbuch).

Es ist viel und heftig darüber diskutiert worden, wie sich die Zählfertigkeit entwickelt und wie es numerische Bedeutung erlangt. Im folgenden Abschnitt werde ich die diesbezügliche Diskussion zusammenfassen, wobei ich mich vor allem an Moser Opitz (2001) orientiere.

\subsection{Die Entwicklung des Zählens}

\subsubsection{Die Prinzipien-Vorher-Theorie}

Auf den Seiten 16ff wurde kurz gezeigt, dass Babys und auch Tiere über Kompetenzen verfügen, die es erlauben, Anzahlveränderungen bei kleinen Mengen wahrzunehmen. Diese Auffassung wird in der neueren Fachliteratur einhellig vertreten. Die Kinder entwickeln ein Bewusstsein für eins, zwei und drei. Interessanterweise können Kleinkinder diese kleinen Anzahlen auch benennen (Wynn 1992a, 317ff in Moser Opitz 2001, 64). Dabei wird in der Fachliteratur oft der Ausdruck „subitizing“ verwendet: ,[...] very young children are said to respond to numerical display by ,subitizing', by reciting a number word they have learned to associate with a given visual pattern, much as they 
learn to associate common nouns with common objects" (Gelman, Meck 1992, 173). Wynn (1992a, 1995, 1997) und Dehaene (1999) haben eingehend die Frage nach der Angeborenheit von numerischen Kompetenzen erörtert. Diese Autoren schließen aus den Ergebnissen ihrer Versuche, dass die Kinder von aller Anfang an eine Vorstellung von Quantitäten besitzen. Aufbauend auf diese Mengenvorstellungen sind die Kinder in der Lage, einfache Additions- und Subtraktionsaufgaben zu verstehen (Wynn 1992a, 322ff). Aus der Einsicht, dass schon viereinhalb Monate alte Babys in der Lage sind, Veränderungen bei kleinen Kollektionen wahrzunehmen ${ }^{8}$, gilt für K. Wynn als gesichert, dass es ein angeborenes numerische Wissen gibt. Nach der Meinung von Gelman u.a. (Gelman 1990; Gelman \& Gallistel 1978; Gelman \& Greeno 1989; Gelman \& Meck 1986) können Kinder Objekte zählen, ohne es je lernen zu müssen. Man müsse ihnen nicht beibringen, dass jedes Objekt nur einmal gezählt werden soll und dass es für die Zahlwörter eine fest vorgegebenen Reihenfolge gibt. Und vor allem: die Kinder wissen, dass die letzte Zählzahl die Anzahl der Objekte einer abgezählten Menge angibt. Die Autoren gehen davon aus, dass es Zählprinzipien gibt, die angeboren sind und nicht gelernt werden müssen: Zählen als eine angeborene Fähigkeit des menschlichen Gehirns. Dieses Konzept wird „Principles-first-Theorie“ und in der deutschsprachigen Literatur „Prinzipien-Vorher-Theorie“ (Stern 1998; Moser Opitz 2001) genannt.

Auf die oben geschilderten Beobachtungen aufbauend schlagen R. Gelman und C.R. Gallistel fünf dem Zählen zugrundeliegenden funktionalen Prinzipien vor (Gelman \& Gallistel 1978, 77 ff):

1. Eineindeutigkeitsprinzip oder Eins-zu-Eins-Zuordnung (one-one principle) Jedem Objekt einer zu zählenden Kollektion wird ein und nur ein Zahlwort zugeordnet.

2. Prinzip der stabilen Ordnung (stable-order principle)

Die beim Zählen benutzten (Zahl-)Wörter müssen in einer stabilen, d.h. stets in gleicher Weise wiederholbaren Ordnung vorliegen und so arrangiert werden, d.h. für jede Quantität steht ein anderes Zahlwort zur Verfügung.

3. Kardinalprinzip (cardinal principle)

\footnotetext{
${ }^{8}$ Das berühmt gewordene Experiment von K. Wynn zeigt, dass die Babys erwarten, dass $1+1$ gleich 2 ist. Zuerst wird eine Mickymaus hinter einer Sichtblende versteckt, dann eine zweite gleiche. Wenn der Schirm weggeklappt wird, sind manchmal beide Figuren zu sehen und manchmal nur eine (die andere wurde heimlich entfernt). Die Kinder schauten systematisch länger auf das unmögliche Ereignis $1+1=1$ als auf das mögliche $1+1=2$, was nahe legt, dass sie zwei Dinge erwarteten (Übersetzung Dehaene 1999, 68).
} 
Das letzte (Zahl-)Wort, das bei einem Zählprozess benutzt wird, repräsentiert die Anzahl der gezählten Objekte, d.h. die Mengengröße wird durch Zählen ermittelt. Zum Kardinalprinzip gehören vier Aspekte:

a. Betonung des letztgenannten Zahlwortes,

b. Wiederholung des letztgenannten Zahlwortes,

c. Feststellen der Anzahl einer Menge, ohne dass ein zweites Mal gezählt wird,

d. Auf Nachfrage zur Anzahl einer Menge, die einmal gezählt wurde, mit dem letztgenannten Zahlwort antworten (vgl. Moser Opitz 2001, 68).

4. Abstraktionsprinzip (abstaction principle)

Die Zählprinzipien 1. bis 3. (die „how-to-count principles“; Gelman \& Gallistel, 1978, 80) können auf jede beliebige Kollektion von Objekten angewendet werden, d.h. Generalisierung des Zählens auf alle Bereich, bei denen diskrete Größen vorkommen.

5. Prinzip von der Irrelevanz der Anordnung (order-irrelevance principle)

Die Anordnung der Objekte einer Kollektion ist für das Zählen (für das Resultat des Zählens) irrelevant, d.h. man kann mit einem beliebigen Objekt mit dem Zählen beginnen. (vgl. Schmidt; Weiser 1982, 233 und Stern 1998, 55).

R. Gelman und C.R. Gallistel (1978) stützen ihre Aussage zudem durch Beobachtungen an 2- bis 3-jährigen Kindern. Kinder in diesem Alter zählen oft fehlerhaft. Zum Beispiel verwechseln sie Zahlwörter oder sie lassen auch mal Zahlwörter aus. Hingegen konnten keine Fehler, die gegen die fünf Prinzipien verstoßen, beobachtet werden. Es wurden in den durchgeführten Versuchen keine Fehler der Art 1, 2, 2, 3, ... oder 1, 2, 3, 2, ... beobachtet (Zählprinzipien 1 und 2). Es gab sogar Proteste seitens der Kinder, auch von Kindern, die noch nicht selber zählen konnten, wenn der Experimentator Fehler dieser Art machte. Das Kardinalprinzip sei angeboren, so schließen Gelman und Gallistel (1978) aus folgenden Beobachtungen: Kinder, die falsch zählten, z.B. 1, 3, 4, 8, antworteten auf die Frage, wie viel Objekte es gibt, mit ,8“. Die Autoren schließen daraus, dass die Kinder wissen, dass die letzte Zahl die Anzahl der Elemente einer Menge beschreibt und dass die Kinder somit über das Kardinalprinzip verfügen (vgl. Stern 1998, 56). Mindestens diese ersten drei Prinzipien („how-to-count principles“) seien verfügbar. Dabei werden diese Prinzipien als Grundlage des Zählens betrachtet und sogar im Sinne von Piaget als Schema definiert. „We believe that our results support the view that these principles constitute a schema in the Piagetian sense. The principles guide and structure 
the child's counting behavior, serve as a reference against which the child can evaluate this actual counting behavior, and motivate that behavior" (Gelman \& Gallistel 1978, 208).

Die Autoren gehen davon aus, dass im ganzen Vorschulalter nicht so sehr die Gewinnung anderer Zählprinzipien im Vordergrund steht, sondern die Erzeugung von zunehmender Sicherheit im Zählen und in der Erweiterung des Zahlenraumes, über den zählend verfügt wird.

Gelman \& Gallistel haben lange Zeit nur diese fünf Zählprinzipien für die Anzahlbestimmung für die Kinder verfügbar gehalten und verwarfen die Quantitätsbestimmung auf Basis von subitizing. Die Autoren führten an, dass Kleinkinder auch bei kleinen Kollektionen zählten, um die Anzahl zu bestimmen, räumten aber ein, dass eine geschickte Anordnung der einzelnen Elemente bei der Anzahlbestimmung hilfreich sei (Gelman \& Gallistel 1978, 220 in Moser Opitz 2001, 69). Die Autoren anerkannten später aber bei präverbalen Zählprozessen subitizing-Vorgänge als Ursprung der Zählprinzipien. „The preverbal process provides a framework that makes the verbal counting process intelligible, hence lernable. Children assimilate verbal counting because it maps onto the unconscious preverbal counting process. The count words map to the preverbal magnitudes." und "The principles that govern verbal counting are the principles implicit in the structure of the preverbal counting mechanism" (Gallistel \& Gelman 1993, 65 und $71)$.

Die von Gelman und Gallistel propagierten fünf Prinzipien haben sich jedoch in den Nachfolgeuntersuchungen bei jüngeren Kindern als sehr instabil erwiesen. Einmal erworbene Zählkompetenzen konnten nicht ohne weiteres auf neue Situationen angewandt werden. Eine Relativierung der bisherigen Sichtweise der „Prinzipien-Vorher-Theorie“ hat Gelman (1997) mit einem rational-konstruktivistischen Ansatz vorgenommen (vgl. Moser Opitz 2001, 69). Eigene Untersuchungen ab Seite 94 konnten diese brüchigen Fertigkeiten bei einigen schon älteren Kindern bestätigen. Selbst bei vier- bis fünfjährigen Kindern ist die Eins-zu-Eins-Zuordnung (Prinzip 1) oder das Kardinalitätsprinzip (Prinzip 3) sehr unsicher vorhanden (siehe auch ab Seite 190).

Diese Theorie „Prinzipien Vorher“ hat vor allem Kritiker und wenig Anhänger gefunden (Fuson 1988; Sophian 1988a; Baroody 1991; Wynn 1990 und 1992a; Frydman 1995a; Parrat-Dayan 1995; Bryant 1997). Es ist aufschlussreich, die verschiedenen Kritikpunkte kurz zu schildern. Die einzelnen Kritikpunkte sind auf der Basis von Moser Opitz (2001, 69ff) zusammengefasst: 
Grundsätzlich wird die Angeborenheit von Konzepten kritisiert. So folgert Wynn (1990, 189) aus ihren Untersuchungen, dass erstes Zahl- und Zählwissen angelernt sei und nicht auf angeborene Kompetenzen basiert. Die Autorin zeigt, dass Kinder unter dreieinhalb Jahren das Kardinalitätsprinzip nicht verstehen würden. Einzig subitizing sei ein angeborenes Konzept. Brissiaud (1995, 699f) führt an, dass Zählen und kulturelle Konzepte eng miteinander verzahnt seien und nicht in der Ontogenese des Menschen enthalten seien. Taube Kinder, so zeigt der Autor, können erst mit einem bestimmten Fingerzählsystem vom einfachen Nachplappern der Zahlwortreihe zu einem echten Verständnis des Zählens kommen. Sophian (1988a, 639) folgert nach Versuchen mit Kindern zwischen drei und vier Jahren, dass das Verständnis des Zählens sich erst durch Erfahrung mit Zählen einstellt. Dieses „Wissen-Warum“ stelle sich erst mit zunehmenden Erfahrungen ein. Zählentwicklung sei nicht durch Prinzipien geleitet.

Nebst dieser grundlegenden Kritik zu Angeborenheit von Konzepten sind die einzelnen der fünf Zählprinzipien in Frage gestellt worden:

Gelman (1990) leitet aus der beobachteten Tendenz von Kindern, jedes Objekt nur einmal zu zählen, das Eineindeutigkeitsprinzip her. Baroody $(1991,146)$ moniert, dass aus diesen Beobachtungen nicht ein Prinzip abgeleitet werden könne. Diese Ein-zu-EinsZuordnung würde sich aus Nachahmung und häufiges Üben entwickeln. Fuson (1988, 123) zeigt, dass die Fähigkeit der Ein-zu-Eins-Zuordnung nicht auf ein schon vorhandenes Konzept beruht, sondern dass die Zählentwicklung ein stetig zunehmendes Wissen und stetig zunehmende Kompetenzen umfasst. Die eineindeutige Zuordnung gelingt nur, wenn Kinder in der Lage sind, vielfältige Aktionen miteinander zu koordinieren: Die Objekte müssen angetippt werden, jedem Objekt muss ein genau festgelegtes Zahlwort zugeordnet werden, kein Objekt darf ausgelassen und keines doppelt gezählt werden. Eigene Untersuchungen bestätigen diese Befunde von Fuson (Siehe ab Seite 199).

Baroody (1991, 141ff.) findet, dass das Beherrschen der Zahlwortreihe vor allem eine Gedächtnis- und Nachahmungsleistung sei und nicht auf einem angeborenen Prinzip der stabilen Ordnung beruhe. $\mathrm{Zu}$ einem gleichen Befund kommt Fuson (1988, 35ff.). Auch sie kommt zum Schluss, dass die Zahlwortreihe von den Kindern konstruiert werde. Die Kinder zählen anfangs „einszweidreivierfünf...“. Erst später lernen sie diese Folge in Wörter zu unterteilen und sie in konkreten Situationen anzuwenden. Baroody (1991, 144 in Moser Opitz 2001, 71) beschreibt den Ablauf: „Zuerst entdecken die Kinder, dass sich die Zahlwortreihe aus bestimmten Wörtern, eben Zahlwörtern zusammensetzt. An- 
schließend wird ihnen bewusst, dass die Wörter in eine bestimmte Reihenfolge gehören und dass jedes Wort nur einmal vorkommt.“

Auch das Kardinalitätsprinzip wurde heftig verworfen. So bemerkt etwa Wynn (1990, 162), dass der Teilaspekt „Betonung des letztgenannten Zahlwortes“ auch einfach das Ende des Zählprozess signalisiere oder durch Nachahmung angelernt sei. Diese Betonung bedeute keineswegs das Vorhandensein des Kardinalprinzips. Wynn stellt zudem fest, dass jüngere Kinder öfter als ältere Kinder auf die Frage „Wie viel sind es?“ nicht das letztgenannte Zahlwort wiederholten, sondern die ganze Kollektion nochmals zählten. Wynn schließt aus diesen Beobachtungen, dass das Kardinalitätsprinzip erst von den älteren Kindern verstanden werde. Darum lehnt Wynn die „Prinzipien-VorherTheorie“ ab. Hier muss aber bemerkt werden, dass auf diese für die Kinder überraschende Frage das Erinnerungsvermögen in ausgeprägter Weise vorhanden sein muss. Es muss die Möglichkeit ins Auge gefasst werden, dass die jüngeren Kinder die letzte Zahl einfach öfters vergessen haben als die älteren. Frydman und Bryant (1988) plädieren auf Grund ihrer Untersuchungsergebnisse, dass das Kardinalitätsprinzip erst aus Erfahrung mit Zählen erschlossen wird. Auch sie stellten fest, dass fünfjährige Kinder über das Kardinalitätsprinzip verfügten, vierjährige nicht. Auch Fuson (1988) hat das Vorhandensein des Kardinalitätsprinzips überprüft. Obwohl die Kinder in ihrer Testreihe die Anzahl Elemente einer Menge mit bis zu sieben Gegenständen richtig ermitteln konnten, zählten sie nochmals, wenn ihnen die Frage „Wie viel sind es?“ gestellt wurde. Gelman (1990) wendet auf die Kritik von Fuson ein, dass die Kinder möglicherweise meinten, etwas falsch gemacht zu haben und darum erneut zählten (vgl. Stern 1998, 57). Eigene Beobachtungen bestärken dieses brüchige Verständnis des Kardinalitätsprinzips (Siehe ab Seite 199).

Vor allem Baroody (1993, 414ff.) stellt das Prinzip der Irrelevanz der Anordnung in Frage. Er stellt fest, dass Kinder zwar im Stande sind, einem gezeigten Gegenstand ein Zahlwort zuzuordnen. In weit geringerem Masse sind sie aber befähigt, Gegenstände in unterschiedlicher Anordnung zu zählen und dabei zu erkennen, dass das Resultat immer dasselbe ist. Nach Meinung von Baroody (1993, 425) können die Kinder zwar richtig zählen ohne aber den Zählvorgang richtig verstehen zu können. Briars und Siegler (1984) beobachteten drei- bis vierjährige Kinder, die beurteilen sollten, ob eine Puppe fehlerhaft zählte oder nicht. Die Puppe machte echte Fehler (Auslassen von Objekten oder Doppelzählung oder korrektes, aber ungewöhnliches Zählen, z.B. indem sie in der Mitte der Objektreihe zu zählen begann oder im ersten Durchgang jedes weitere Objekt 
und im nächsten Durchgang die restlichen Objekte zählte). Kinder unter vier Jahren behaupteten öfters als ältere Kinder, dass diese Vorgehensweise falsch sein - was natürlich nicht stimmte.

Gelman (1990) entgegnete, dass diese korrekten, aber ineffizienten Zählmethoden von keinem Kind spontan gewählt würden, weil funktionale Einschränkungen nicht nur falsche, sondern auch ineffiziente Vorgehensweisen verhindern (vgl. Stern 1998, 56).

\subsubsection{Prinzipien-Nachher-Theorie}

Wie aus den Kritikpunkten der Prinzipien-Vorher-Theorie hervorgeht, muss davon ausgegangen werden, dass wenigstens einige Prinzipien aus Erfahrung mit dem Zählen erschlossen werden. Die Basis für diese alternative Theorie (Prinzipien-NachherTheorie) legte K.C. Fuson (1982). Aus deren Sichtweise müssen verschiedene Faktoren zusammenspielen, bis das Kind über eine gefestigte Zählkompetenz verfügt. Die Autorin unterscheidet fünf Phasen bei der Ausdifferenzierung der Zahlwortfolge (In: Schmidt; Weiser 1982, 234ff)

(I) Phase der noch nicht differenzierten Ganzheitsauffassung der Zahlwortfolge (string_level):

Die einzelnen Zahlwörter der jeweils beherrschten Zahlwortfolge werden nicht als abgrenzbare Teile derselben aufgefasst, vielmehr wird über die Zahlwortfolge als einer noch undifferenzierten Ganzheit (als Lied, als Gedicht) verfügt. Ein Auszählen einer Kollektion von Dingen auf dieser Ebene ist noch eine ,globale Korrespondenz“" zwischen den Zahlwörtern und den Dingen, die Erfüllung der Eindeutigkeit ist mehr zufällig als systematisch.

(II) Phase der differenzierten Ganzheitsauffassung der Zahlwortfolge (unbreakable chain_level):

Die einzelnen Zahlwörter der jeweils beherrschten Zahlwortfolge werden als einzelne abgrenzbare Teile unterscheidbar; freilich muss noch die gesamte Zahlwortfolge von Anfang an reproduziert werden, z.B. wenn das auf „sechs“ unmittelbar folgende Zahlwort ermittelt werden soll.

In dieser Phase gelingen innerhalb der beherrschten Zahlwortfolge kardinales Auszählen (wie viele?) und Abzählen (Stelle durch Zählen eine Kollektion von $n$ Dingen her!) sowie ordinales Zählen (an wievielter Stelle?). Die Kleiner- bzw. 
Größerrelation wird durch „,...kommt (direkt) vor- “ bzw. „... kommt (direkt) nach- " gebildet, sie kann jedoch nur durch Reproduktion der Zahlwortfolge von Anfang an realisiert werden. Die kardinale Addition wird durch Auszählen der Summenkollektion (Zusammenzählen) von „Anfang an“ bewältigt.

(III) Phase des Weiterzählens-Könnens (breakable chain):

Es kann nunmehr von einer Stelle an weitergezählt werden, ein Start bei „1“ ist nicht mehr unbedingt nötig; es kann auch von $a$ bis $b$ gezählt werden. Dazu kommt das Rückwärtszählen von einer Stelle an und von $b$ bis $a$.

Die Kleiner- bzw. Größer-Relation kann jetzt unmittelbar durch: „,... kommt (direkt) vor- “ bzw. „,... kommt (direkt) nach-“" realisiert werden, ohne von eins an zu zählen. Weiterzählen und Rückwärtszählen werden jetzt benutzt zur kardinalen Addition und Subtraktion, jedoch noch ohne Sicherheit bezüglich der Ermittlung der Anzahl der Schritte beim Weiterzählen bzw. Rückwärtszählen.

(IV) Phase der Auffassung der Zahlwörter als zählbare Einheiten (numerable chain level):

Die einzelnen Zahlwörter werden nicht nur als wohlunterschiedene, sondern auch als selbst hinwiederum zählbare Einheiten betrachtet, so dass nunmehr auch Teilstücke der beherrschten Zahlwortfolge ausgezählt werden können.

Es gelingt das Weiterzählen (Rückwärtszählen) von $a$ um $n$, das Weiterzählen (Rückwärtszählen) von $a$ nach $b$ und die Ermittlung der Anzahl der Zahlwörter von $a$ nach $b$. Zwischen Weiterzählen und Rückwärtszählen liegt eine gewisse Zeitspanne - letzteres entwickelt sich etwa um das 7. Lebensjahr.

Beim Addieren und Subtrahieren kommt es zur Sicherheit bei der Ermittlung der Anzahl der Schritte beim Weiter- , bzw. Rückwärtszählen.

(V) Phase der ,zweiseitigen Durchlaufbarkeit“ der Zahlwortfolge (bidirectional chain level):

Nun kann die beherrschte Zahlwortfolge flexibel und schnell von ,jeder“ Stelle aus vorwärts wie rückwärts durchlaufen werden. Vorwärts- wie Rückwärtszählen werden flexibel und schnell gebraucht sowie kombiniert.

Der Stand der Zählentwicklung kann nur vollständig erfasst werden, wenn er in verschiedenen Situationen, die unterschiedliche Probleme und somit auch unterschiedliche Anforderungen enthalten, betrachtet wird. Aufbauend auf die fünf Phasen von Fuson 
betrachtet Wynn (1992b, 223) die drei Begriffe Sequenz, Zählen und kardinale Bedeutung als zentral:

- Sequenz: Rezitation der Zahlwortreihe, ohne dass damit vorhandene oder nicht vorhandene Elemente gezählt werden. Ein Zahlwort ist Teil einer rezitierten Folge.

- Zählen: Beinhaltet als neuen Schritt das Zählen von Elementen: Jedem Element wird durch Ein-zu-Eins-Korrespendenz genau ein Zahlwort zugeordnet.

- Kardinale Bedeutung: Das Kind erkennt, dass ein bestimmtes Zahlwort eine bestimmte Anzahl Elemente bezeichnet. Dieses Verständnis wird erst möglich, wenn das Kind die Zahlwortreihe beherrscht und Objekte zählen kann (vgl. Moser Opitz 2001, 73).

Allgemein herrscht heute Konsens, dass sich Kinder die unterschiedlichen Bedeutungen und Aspekte des Zählens erst nach und nach aneignen. Fuson (1988), Baroody (1991) und Resnick (1989) gehen davon aus, dass der Zählentwicklungsprozess durch Interaktion mit Eltern, Geschwister und Spielkameraden stark gefördert wird. Das erste Zählen sei eine Routinetätigkeit, bei der Erwachsene oder eben Geschwister und Spielkameraden nachgeahmt werden und die Kinder müssten die unterschiedlichen Kompetenzen, die zum Zählen erforderlich sind, in verschiedenen Kontexten lernen, um so die allgemeinen Zählprinzipien zu entwickeln. Wynn (1992a, 1995) ist hingegen vermehrt der Idee des subitizing zugetan. Subitizing heißt Mengenschätzung, d.h. spontane Erfassen der Größe konkreter Mengen mit bis zu vier Gegenständen (vgl. Ginsburg 1977; Saxe \& Gearhart 1988; Stern 1998; Moser Opitz 2001). Für die Autorin ist dieses wahrnehmungsmäßige, schnelle Bestimmen einer kleinen Kollektion von zentraler Bedeutung, in der Hinsicht, dass Kinder bis zum Alter von drei Jahren zählen, indem sie angeborene Prinzipien wie dieses subitzing verwenden und erst später den Zusammenhang zwischen dem Zählen und der Anzahl der gezählten Objekte zu verstehen beginnen. In ähnlicher Weise argumentiert Geary (1995), indem er von biologisch ersten und biologisch zweiten kognitiven Fähigkeiten spricht. Während die biologisch ersten Fähigkeiten (im Hinblick auf die Mathematik unterscheidet er Vielfachheit [numerosity], Ordnung [ordinality], Zählen und einfaches Rechnen mit höchstens vier Objekten) offenbar in allen Kulturen gefunden werden (siehe Seite 14), scheinen sich die biologisch zweiten Fähigkeiten (für die Mathematik nennt Geary Zählen und Zahl sowie das Rechnen) nur in spezifischen kulturellen Kontexten zu entwickeln und es dürfte klar sein, dass die späteren (die zweiten) mathematischen Kompetenzen von Eltern, Geschwistern, Spielkame- 
raden, in der Schule, im Fernsehen usw. gelernt werden. Speziell beim Zählen ist im Alter von etwa zwei bis sieben Jahren, und - wie wir noch zeigen können - insbesondere zwischen dem vierten und sechsten Lebensjahr, eine deutliche Entwicklung zu erkennen. Diese Entwicklung beginnt mit dem Nachsagen und Erkennen der Zahlwörter im Alter von etwa zwei Jahren, wobei das Kind aber noch nicht weiß, was diese Zahlwörter eigentlich bedeuten. Es folgt dann die Periode des (a)synchronen Zählens, d.h. die Kinder beginnen Objekte abzuzählen, zählen aber Objekte mehrfach und vergessen einige, wobei auch hier - wie wir zeigen können - zwischen dem vierten und sechsten Legensjahr eine deutliche Entwicklung stattfindet. Anschließend, wenn die Kinder etwa fünf Jahre alt sind, kommt die Periode des resultativen Zählens, d.h. die Kinder wissen, dass sie, wenn sie zählen, mit eins beginnen und jedes Objekt genau einmal zählen müssen; und sie haben das Kardinalitätsprinzip verstanden, d.h. sie verstehen, dass das letzte Zahlwort die Gesamtzahl der Objekte angibt (Gelman \& Gallistel 1978; Ginsburg 1977; vgl. auch van Luit, van de Rijt, Hasemann 2000).

Die Prinzipien-Nachher-Theorie findet breiteste Zustimmung. Die weitere Untermauerung dieser Theorie ist in den einzelnen Kritikpunkten der Prinzipien-Vorher-Theorie zu finden (vgl. Moser Opitz 2001, 73). 


\section{Kompetenz, Performanz und Kognition}

\subsection{Kompetenz und Performanz}

\subsubsection{Allgemeine Betrachtungen}

Wohl aus dem Umstand, dass sich in der Literatur keine genauen Definitionen über die beiden Begriffe „Fähigkeit“ und „Fertigkeit“ finden lassen, werden seit einigen Jahren in der kognitionspsychologischen Diskussion diese beiden Ausdrücke gemieden und zum Teil ersetzt durch die Begriffe „Kompetenz“ und „Performanz“. So sind in der aktuellen Literatur Sätze wie „Die Fertigkeit ist, was jemand in der Lage ist zu tun, d.h. die Fähigkeit, bestimmte Handlungen oder Folgen von Handlungen (motorische oder kognitive) auszuführen“ (Wachsmuth 1985, 7; Kursivschrift im Original) nicht mehr zu finden. Im Alltag werden diese zwei Wörter „Fertigkeit“" und „Fähigkeit“ aber nach wie vor oft gebraucht in dem obgenannten Sinne, dass sich der Begriff „Fertigkeit“ auf die Beherrschung von Handlungsvollzügen unter Einfluss der notwendigen Genauigkeit, Geschwindigkeit, Bewegungskoordination (z.B. die Abstimmung von linker und rechter Hand) bezieht; der Begriff „Fähigkeit“ bezieht sich auf allgemein verwendbare Regeln (Pläne und Strategien) zur Problemlösung. Es herrscht Konsens in der Auffassung, dass die kognitiven Anforderungen der „Fähigkeiten“ über denen von „Fertigkeiten“ stehen. Die Unterscheidung zwischen den beiden Begriffen „Kompetenz“ und „Performanz“ geht auf Noam Chomsky zurück, der sie in seinem bedeutenden Werk „Aspekte der Syntax-Theorie“ geprägt hat. Unter Kompetenz versteht er die „Kenntnis des Sprechers [...] von seiner Sprache“ (Chomsky 1973, 14). Damit meint er das gesamte grammatikalische und lexikalische Wissen über eine Sprache, das im Gehirn eines Sprechers gespeichert ist und worauf das er theoretisch bei der Produktion sprachlicher Äußerungen zurückgreifen kann. Dies gelingt aber nur theoretisch; denn tatsächlich ist nur der von Linguisten viel bemühte, hypothetische „ideale Sprecher“ (Chomsky 1973, 13) in der Lage, seine Äußerungen ständig in völliger Übereinstimmung mit seinem psycholinguistischen Wissen zu produzieren, so dass „die Sprachverwendung als direkte Widerspiegelung der Sprach-Kompetenz aufgefasst werden kann“(Chomsky 1973, 14).

Jeder reale, menschliche Sprecher ist hingegen bei seiner wirklichen Sprachverwendung, der von Chomsky so genannten „Performanz“ (Chomsky 1973, 14), einer Vielzahl von Einflüssen unterworfen, die es ihm unmöglich machen, sich allein und uneinge- 
schränkt auf sein psycholinguistisches Wissen zu stützen. Dazu zählt Chomsky u.a. „Zerstreutheit und Verwirrung“, „Verschiebung in der Aufmerksamkeit und im Interesse“ und ein „begrenztes Gedächtnis“ (Chomsky 1973, 13). Gerade der letztgenannte Aspekt scheint bei der spontanen, mündlichen Sprachproduktion von besonderem Belang zu sein; denn wo beim Verfassen schriftlicher Texte die begrenzte Gedächtnisleistung durch längere Bedenkzeit zumindest teilweise ausgeglichen werden kann, da steht der Sprecher in der mündlichen Kommunikation unter Zeitdruck und er kann nichts vom schon Geäußertem zurücknehmen und geschickter formulieren.

Diese von Chomsky vorgeschlagene Definition der zwei Begriffe „Kompetenz“ und „Performanz“ eignen sich nun ebenfalls für die Analyse von mathematischen Leistungen.

Verallgemeinert und vereinfacht kann man den Sachverhalt nämlich wie folgt darstellen: die Kompetenz ist das, was ein Individuum in einem Inhaltsbereich prinzipiell kann und weiß, sein Potential; die Performanz ist dann die eingeschränkte Anwendung der Kompetenz. Bei der Bewertung von Tests und psychologischer Untersuchungen über die kognitive Leistungsfähigkeit von Versuchspersonen ist es klar, dass die gezeigten Leistungen nur die beobachtbare und messbare Performanz, nicht aber die tatsächliche Kompetenz der Individuen widerspiegeln. Es ist darum nicht immer ersichtlich, was denn bei Versuchspersonen die Kompetenz ist und ob oder wie sich diese Kompetenz in der Performanz äußert, d.h. wie weit es einer Versuchsperson gelingt, ihre Kompetenz über die Performanz zu dokumentieren. Während zum Beispiel einem Kindergartenkind unterstellt wird, dass es noch nicht das zum Lösen einer Aufgabe (z.B. das Bestimmen der Mächtigkeit einer ungeordneten überschaubaren Menge von Würfeln) benötigte Wissen verfügbar hat, wird bei einem Viertklässler bei der Nichtbewältigung derselben Aufgabe davon ausgegangen, dass er, als ihm die Aufgabe gestellt wurde, nicht von seinem verfügbaren Wissen und seinen Kompetenzen Gebrauch gemacht und deshalb nicht die optimale Leistung erbracht hat. Schon seit langem wird darum auf das Problem hingewiesen, wie denn die Testergebnisse einer Untersuchung einzuordnen sind. Wird da die Kompetenz gemessen oder nur, wie diese Kompetenz genutzt wird, d.h. die darauf aufbauende Performanz? Der Fachdiskussion darüber ist nicht leicht zu folgen, da die Begriffe oft uneinheitlich verwendet oder nicht geklärt werden. Im Folgenden wird versucht, diese Diskussion zusammenzufassen, um ein einheitliches Bild über die oben erwähnte Problematik zu erhalten. 
Davidson und Sternberg (1985) weisen dezidiert auf dieses Problem in den Untersuchungen zum Problemlöseverhalten und zum Zahlengedächtnis von Kindern hin: „However, experimental results that purport to show children's intellectual levels often are ambiguous as to whether the observed levels reflect the children's competence or, instead, their ability to use this competence" (Davidson/Sternberg, 1985, 43). Während am obigen Beispiel dem Kindergartenkind also ein Mangel an Kompetenz unterstellt wird, gesteht man dem Viertklässler bei der Nichtbewältigung derselben Aufgabe nicht ein Mangel an Kompetenz, sondern an Performanz zu (vgl. auch Stern 1998, 17).

\subsubsection{Theoretische Modelle und Hintergründe}

Stern $(1998,18)$ versteht unter Kompetenz die Verfügbarkeit von Wissen, mit dessen Hilfe die in einer Situation gestellten Anforderungen erkannt und bewältigt werden können. Die Autorin geht davon aus, dass sich dann Aussagen zur Performanz auf geleistete Problemlösungen beziehen und Auskunft darüber geben, ob ein Individuum einer bestimmten Anforderung gerecht wurde oder nicht. Der Begriff Kompetenz wird benutzt, um Aussagen über das Verhalten in potentiellen Anforderungssituationen zu machen. Kompetenzen können aber nur aus der direkt beobachtbaren Performanz erschlossen werden. Die Tatsache, dass eine Person eine hohe Performanz in einer bestimmten Anforderungssituation gezeigt hat, erlaubt Schlussfolgerungen über ihre Kompetenz in ähnlichen Anforderungssituationen. Die Autorin bezeichnet mit den Begriffen Reifung, Entwicklung und Lernen Vorgänge, die ein Individuum in die Lage versetzen, diese externen Anforderungen zu bewältigen. Anforderungen stellen sich in einem ganz konkreten Kontext, also in einer Situation. Wird ein bestimmtes mathematisches Problem als Stillarbeit gegeben, stellt dies eine andere Anforderungssituation dar als das Lösen derselben Aufgabe an der Wandtafel vor den Augen der ganzen Klasse. Zur Bewältigung von Anforderungen müssen folgende Voraussetzungen erfüllt sein:

- die perzeptiven (wahrnehmend) und repräsentationalen Voraussetzungen zur Verarbeitung der entscheidenden Merkmale der Anforderungssituation (repräsentationalen Voraussetzungen),

- die Voraussetzungen zur Ausführung der erforderlichen motorischen - beim Menschen auch sprachlichen - Handlungen (aktionale Voraussetzung) und 
- die Möglichkeit zur Herstellung einer Verbindung zwischen der repräsentationalen Anforderungssituation und der zu ihrer Bewältigung zu zeigenden Handlung.

Für E. Stern ist die repräsentationale Kompetenz die Verfügbarkeit von spezifischem Wissen und die aktionale Kompetenz die Verfügbarkeit von motorischen Abläufen. Für die Bewältigung fast aller Anforderungen benötigt man repräsentationale und aktionale Kompetenzen. Als Beispiel seinen hier das Fahrradfahren, die Türme von Hanoi, Mikado, Skifahren, Tennis oder Schachspielen erwähnt.

Das entscheidende Merkmal für das Vorhandenseins von repräsentationalen Kompetenzen bei einem Individuum besteht in dem zielgerichteten Ordnen der externen Informationen im Hinblick auf die Bewältigung einer Anforderung. Auch wenn zur Bewältigung fast aller Anforderungen kognitive und aktionale Voraussetzungen benötigt werden, ist bei vielen Situationen eine der beiden Kompetenzen vollständig automatisiert, d.h. entweder die repräsentationale oder aber die aktionale Voraussetzung wird überhaupt nicht mehr zur Kenntnis genommen, ist einfach ohne Anstrengung verfügbar. Es gibt aber Anforderungen, in denen gleichermaßen hohe aktionale und repräsentationale Kompetenzen vorausgesetzt werden (Fremdsprachen, Tastaturschreiben, Tennisspielen, ...). Wird, um beim obigem Beispiel zu bleiben, einem vierjährigem Kind die Aufgabe gestellt, die Anzahl einer ungeordneten, aber überschaubaren Menge von Würfeln zu bestimmen, so erfordert die Lösung dieser Aufgabe repräsentationale Kompetenzen, die von aktionalen Kompetenzen unterstützt werden. Ein mögliches Vorgehen besteht darin, jeden Gegenstand anzufassen, ein Zahlwort zu sagen und ihn zur Kennzeichnung der bereits erfassten Gegenstände beiseite zu schieben. Bei einem vierjährigen Kind ohne Sprach- und Motorikstörungen wird die Bewältigung dieser Anforderung nicht an den aktionalen Anforderungen, d.h. am Aussprechen der Zahlwörter oder am Ergreifen und Bewegen der Gegenstände, scheitern. Fehler treten auf, wenn die Zahlwörter nicht in der richtigen Reihenfolge abgerufen werden oder wenn einige Gegenstände doppelt oder überhaupt nicht gezählt werden, weil die bereits gezählten und die ungezählten nicht oder nicht systematisch durch Veränderung der räumlichen Position voneinander unterschieden wurden (Siehe eigene Untersuchungen z.B. ab Seite 199). Die erfolgreiche Bewältigung der Anforderung „Bestimmen der Mächtigkeit von ungeordneten Gegenständen“ wird erleichtert durch die Nutzung der in der speziellen Situation gegebenen Möglichkeit, die räumliche Position bereits gezählter Gegenstände zu verändern (vgl. Stern 1998, 17-22). 
Die Unterscheidung von Kompetenz und Performanz und die Annahme, diese als gleichberechtigte Faktoren in der kognitiven Entwicklung zu betrachten, verdeutlicht die praktisch pädagogische Relevanz der Berücksichtigung beider Faktoren. Die These, dass die Test- oder Lehrperson direkten Zugang immer nur zur Performanz der Testpersonen resp. seiner Schüler hat, wirft Fragen nicht nur für die Leistungsbewertung, sondern für das methodische Vorgehen überhaupt auf. Wie schon kurz erwähnt, existiert eine Diskrepanz zwischen dem, was ein Individuum seinem kognitiven Entwicklungsstand nach in einer stressfreien idealen Umgebung weiß oder kann und dem, was es in einer oft künstlichen, angespannten und nervösen Testsituation zeigen kann. Aus diesem Grunde ist es nach wie vor schwierig, Ergebnisse psychologischer Experimente oder mathematischer Tests exakt zu interpretieren. Wenn zusätzlich noch verschiedene Personen die Experimente oder Tests ausführen, sind die Resultate auch bei gleichen Voraussetzungen der Probanden stark vom persönlichen Auftreten der Tester mit ihrer individuellen Nuacierung der Durchführung der Untersuchungen abhängig. Das macht es sehr schwierig zu beurteilen, wie stark das tatsächliche Wissen des Probanden zum Vorschein kommt. Von der genauen Erforschung dieser Problematik verspricht man sich wichtige Erkenntnisse für die psychologische Theorie, die pädagogische Praxis ebenso wie für die Testauswertungen.

Es soll nun an dieser Stelle kurz die Begriffe Kompetenz und Performanz aus anderer Sicht geklärt, sowie deren Verhältnis und Wechselwirkung untersucht werden. Die folgenden Ausführungen zur Begriffsbestimmung und zu den Faktoren von Kompetenz und Performanz beziehen sich im Wesentlichen auf die Vorschläge von Davidson/Sternberg (1985).

Ähnlich wie Stern (1998, 17-22) definieren die obgenannten Autoren Kompetenz als Verfügbarkeit von Wissen zur Bewältigung von Anforderungen: „By competence, we refer to the availability of skills and logical structures“ (Davidson/Sternberg 1985, 44). Dazu gehören Informationsverarbeitungsprozesse, Wissen, Kapazität des Arbeitsspeichers und Repräsentationsformate zur Informationsspeicherung. „By performance, we refer to the utilisation of competence" (Davidson/Sternberg 1985, 44). Entscheidender Faktor ist dabei die Effizienz im Zugang zu den unter dem Begriff der Kompetenz beschriebenen Faktoren und Mechanismen. Im Weiteren spielen zusätzlich Motivation, unterschiedliche kognitive Stile und weitere äußere Faktoren eine wichtige Rolle. Im Folgenden sollen die Faktoren, welche Kompetenz und Performanz determinieren, dargestellt werden. $\mathrm{Zu}$ beachten ist, dass die einzelnen Faktoren sowie Performanz und 
Kompetenz im Allgemeinen sehr komplexen Wechselwirkungen unterliegen, so dass es in der Praxis schwer ist, die einzelnen Komponenten genau zu trennen. Außerdem ist zu bemerken, dass, ausgehend von der These, dass Performanz die Äußerung von Kompetenz ist, die Faktoren der Kompetenz nur indirekt zugänglich sind, sich also der direkten Untersuchung verschließen. Die Auffassungen über Festlegung der einzelnen Komponenten und ihre Taxonomisierung gehen allerdings noch weit auseinander (vgl. Mandl 1992, 16).

Davidson/Sternberg geben fünf wichtige Kompetenzfaktoren an: „This account deals with five factors that we believe (...) constitute the development of intellectual competence: availability of information processes, automatization of information processes, availability of knowledge, functional capacity of working memory and representational capacity" (Davidson/Sternberg, 1985, 48). Es wird also zunächst zwischen zwei wesentlichen Komponenten unterschieden: Prozessen und Wissen.

Prozesse bezeichnen die menschliche Fähigkeit, mentale Aktivitäten zu planen, zu koordinieren, darzustellen und aufzurufen. Dabei wird zwischen metastrategischen und strategischen Prozessen unterschieden.

Die drei wichtigsten strategische Prozesse sind Encodieren, Inferenz und Mapping- Prozesse. Auf diese kann bei der Ausführung unterschiedlichster Aufgaben zurückgegriffen werden. Beim Encodieren geht es darum, das Problem aufzunehmen, wesentliche Aspekte im Arbeitsgedächtnis zu speichern und relevante Informationen aus dem Langzeitgedächtnis abzurufen. Inferenz betrifft die Herstellung von Beziehungen zwischen verschiedenen konkreten oder abstrakten Objekten. Beim Mapping werden Relationen zwischen einer neu enkodierten und einer früher enkodierten Struktur hergestellt und diese auf Analogien hin überprüft.

Den metastrategischen Prozessen wird beim Problemlösen eine immer größere Rolle zugeschrieben. „In der aktuellen Forschungsliteratur zu den Voraussetzungen effektiven Lernens, Denkens und Problemlösens wird seit etwa Anfang der 70er Jahre der Stellenwert metakognitiver Wissensbestände und Regulationskompetenzen für die erfolgreiche Aufgabenbewältigung besonders unterstrichen“ (Kunz/Drewniak 1992, 213). Metastrategische Prozesse bezeichnen das Wissen über die vorhandenen Prozesse, also die Vorausplanungen beim Problemlösen, die Fähigkeit Hypothesen aufzustellen, diese mental durchzuspielen, zu möglichen Lösungen zu kommen und diese bei Bedarf wieder zu revidieren. Folgende Faktoren können dabei eine Rolle spielen:

- Verstehen, was eigentlich das Problem ist 
- Selektion der nötigen Prozesse

- Organisation relevanter Informationen

- Vollständiges oder nicht vollständiges (mentales) Durchspielen der einzelnen Prozesse

- globale Planung des Problemlöseprozesses. Bewusstmachung der zur Verfügung stehenden Ressourcen

- Strukturierung gemachter Erkenntnisse mit Blick auf das Ziel

- Bewusstmachung und Nutzung der Rückkopplung (feedback), also das Einbeziehen gemachter Erkenntnisse in neue Lösungsansätze.

Strategischen wie metastrategischen Prozesse laufen häufig automatisch ab, wobei man sich einig ist, dass die Bewusstmachung von Strategien und Prozessen die Lernleistung entscheidend beeinflussen (siehe auch van der Meer 1990, 134).

Da sich Davidson/Sternberg zum Wissen nicht weiter äußern, soll hier noch kurz auf die Unterscheidung von deklarativem und prozeduralem Wissen eingegangen werden: „Toward the goal of characterising knowledge, a distinction is often made between two different types of knowledge: knowing how and knowing that, or procedural and declarative knowledge" (Rabinowitz/Goldberg 1995, 225).

Es wird also unterschieden zwischen deklarativem und prozeduralem Wissen. Zum deklarativen Wissen gehören Fakten und Konzepte (beispielsweise die Tatsache, dass man mit Zählen die Mächtigkeit einer Menge bestimmen kann), von denen man ausgeht, dass sie in semantischen Netzwerken repräsentiert sind. Für Frydman (1995a, 657ff, 1995b, 768) ermöglicht Kompetenz, dass Beziehungen zwischen verschiedenen Handlungen hergestellt und verstanden werden können, dass also die Bedeutung eines Vorganges erfasst wird. Frydman schlägt vor, dieses deklarative Wissen als konzeptuelle Kompetenz oder mit „know-why“ zu bezeichnen. „Kompetenz“ wird also synonym verwendet mit „konzeptueller Kompetenz“. Demgegenüber ist prozedurales Wissen das Wissen um verfügbare strategische und metastrategische Prozesse. Nach Frydman (ebd.) bezeichnet prozedurales Wissen prozedurale Kompetenz und meint damit das Verstehen und Ausführen konkreter Problemlösungen (zum Beispiel die Zählhandlung zur Bestimmung einer Anzahl von Elementen einer Menge) und schlägt dafür den Begriff „,know-how“ vor (vgl. Moser Opitz 2001, 76). Eine kleine Skizze fasst das Besprochene zusammen: 


\begin{tabular}{|c|c|c|c|}
\hline \multicolumn{4}{|c|}{$\begin{array}{c}\text { Kompetenz } \\
\text { (nach Davidson/Sternberg; Rabinowitz/Goldberg; Frydmann) }\end{array}$} \\
\hline \multicolumn{2}{|c|}{ Prozesse } & \multicolumn{2}{|c|}{ Wissen } \\
\hline $\begin{array}{l}\text { Strategische } \\
\text { Prozesse sind: }\end{array}$ & $\begin{array}{l}\text { Metastrategische } \\
\text { Prozesse sind: }\end{array}$ & $\begin{array}{l}\text { Deklaratives } \\
\text { Wissen: }\end{array}$ & $\begin{array}{l}\text { Prozedurales } \\
\text { Wissen }\end{array}$ \\
\hline Encodierung & \multirow{3}{*}{$\begin{array}{l}\text { - Problemverständnis } \\
\text { - Prozessselektion } \\
\text { - Infoorganisation } \\
\text { - Durchspielen der } \\
\text { Prozesse } \\
\text { - Planung } \\
\text { - ... (siehe Text) }\end{array}$} & \multirow{3}{*}{$\begin{array}{l}\text { „know-why“ } \\
\text { Fakten-Wissen } \\
\text { Konzept-Wissen }\end{array}$} & \multirow{3}{*}{$\begin{array}{l}\text { „know-how“ } \\
\text { Wissen um stra- } \\
\text { tegische und } \\
\text { metastrategische } \\
\text { Prozesse }\end{array}$} \\
\hline Inferenz & & & \\
\hline Mapping & & & \\
\hline
\end{tabular}

Abb. 1 Kompetenz

Des öftern wird der Begriff „Performanz“ synonym mit „,praktischer und prozeduraler Kompetenz“" verwendet (Sophian 1997a, 296; Frye 1997, 318 in Moser Opitz 2001, 76). Dabei wird betont, dass zur Performanz ein intentionaler Akt gehört, d.h. eine bewusste und absichtliche Handlung, die zu einem bestimmten Verhalten führt, um ein bestimmtes Ziel zu erreichen. Wie schon angesprochen, ist das entscheidende Kriterium für die Performanz die Effizienz der Ausführung der unter Kompetenz gefassten Prozesse und der Zugriff auf vorhandenes Wissen. Variablen dabei sind Zugänglichkeit, Geschwindigkeit und Korrektheitsgrad. Besondere Bedeutung wird der Aktivierung von relevantem Vorwissen sowie dessen Zusammenspiel mit den zur Verarbeitung notwendigen Prozessen zugeschrieben: „Competent cognitive performance is characterised by easy and fast access to task relevant information“ (Hagendorf 1990, 56). Und weiter: „We believe that strategies and prior knowledge often complement each other to produce competent performance and specifically that efficient cognition can be engineered by promoting use of strategies and prior knowledge" (Pressley/Wood/Woloshyn 1990, 200). Optimale Performanz entsteht in komplexem Zusammenwirken der bisher beschriebenen Prozesse und Strategien der Kompetenz. Da diese aber erst in der praktischen performativen Ausführung aktiv werden, ist es gerade das Zusammenspiel sowie die umfangreichen Wechselwirkungen und gegenseitige Bedingtheit von Kompetenz und Performanz, welches interessante didaktische Ansätze liefern könnte. 
In der kognitiven Entwicklung kommt es zum Phänomen der Rückkopplung (feedback). Das über die Performanz erworbene deklarative und prozedurale Wissen beeinflusst Kompetenz und dies bewirkt in einem kognitiven Lernprozess die Ausprägung und Entwicklung der Kompetenz. Ebenfalls wird deutlich, dass es auch innerhalb der Kompetenz- und Performanzvariablen ${ }^{9}$ Wechselwirkungen gibt. Kurz: Es entsteht durch die Rückkoppelung von „Performanz“ neue „Kompetenz“. Dies betonen auch Schneider/Weinert: „Specific knowledge affects the acquisition of strategies, and (...) strategy deployment in turn influences the construction of specific knowledge" (Schneider/Weinert 1990, 291).

\subsubsection{Praktische Bedeutung}

Aus den bisherigen Darlegungen können bereits einige Konsequenzen vor allem für die pädagogische Praxis, aber auch für Testkonzeptionen gezogen werden. Wichtige Erkenntnis ist zunächst, dass die Testperson resp. die Lehrperson direkten Zugriff nur auf die Performanz der Getesteten resp. der Schüler hat. Diese Performanz entsteht aber nur durch die notwendigen Komponenten (siehe oben) der Kompetenz. Jedoch ist Kompetenz noch nicht hinreichend für die Performanz und entsteht außerdem erst selbst durch diese. Die dabei ablaufenden Prozesse sind sehr komplex und es zeigt sich auch hier, dass Lernen und Lehren also mehr sein muss als ein bloßes Reiz-Reaktions-Schema.

Diese Erkenntnisse sollten übrigens direkte Auswirkungen auf die Lehrkunst haben: Eine gute Didaktik muss die ablaufenden Prozesse berücksichtigen und sich diese zu Nutze machen. Dabei müsste sie möglichst lernerzentriert sein, denn die beschriebenen Prozesse spielen sich im Lerner ab. Dies stellt auf ein Neues den traditionellen ausschließlichen Frontalunterricht in Frage. Weiter unten wird dargelegt, dass große Übereinstimmung herrscht in der Auffassung, dass Kompetenz im Kreislauf aus ihrer performativen Anwendung und feedback entsteht. Die Konsequenz daraus ist, dass die Aktivität zum Lerner hin verlagern werden sollte.

Immer wieder unterstrichen wird die Rolle des Vorwissens. Es muss stets auf vorhandener Kompetenz und „Weltwissen“ aufbauen, dieses Wissen und die notwendigen Prozesse anwenden und somit erweitern. Ebenfalls von großer Bedeutung ist der Einfluss

\footnotetext{
${ }^{9}$ Kompetenzvariablen sind z.B. Prozesse der Informationsverarbeitung, Wissen, Kapazität des Arbeitsspeichers sowie Repräsentationsformate zur Informationsspeicherung Performanzvariablen sind z.B. die Effizienz der unter der Kompetenz gefassten Prozesse und die Zugriffgeschwindigkeit sowie Zugriffskorrektheit und Zugänglichkeit auf vorhandenes Wissen.
} 
der Bewusstmachung der Lernprozesse und Strategien auf die Lernleistung. Eine nachhaltige Didaktik müsste sich dies zunutze machen. Auch für die Leistungsbewertung ergeben sich Konsequenzen. Hier gilt in der Schule der Anspruch, objektiv die Kompetenz des Schülers zu bewerten, ebenso wie bei Untersuchungen die Testergebnisse im Allgemeinen die Kompetenzen widerspiegeln wollen. Dies ist aber, wie wir gesehen haben, nur begrenzt möglich. Die Mechanismen zur Kontrolle von Leistungen müssten also neu durchdacht werden. Lernen ist ein prozessgesteuerter kognitiver Vorgang, der im Wesentlichen über das Wechselspiel zwischen vorhandenem Wissen, neuen Informationen und Prozessen/Strategien zur Informationsverarbeitung gesteuert wird. Die kognitive Kompetenz kann dabei nicht als von sozialer Interaktion isolierter Akt betrachtet werden. Sie manifestiert sich nur über praktische Performanz und erhält durch diese Rückkopplungen.

Vor allem Fuson (1995), Gelman (1997), Sophian (1997a und 1997b) und Siegler (1997) vertreten dezidiert die Meinung, dass diese Interaktion von Kompetenz und Performanz betrachtet werden müsse, dass sich Kompetenz und Performanz gegenseitig beeinflussen und dass gerade diese Wechselwirkung einen wesentlichen Aspekt von Entwicklung ausmacht. Fuson $(1995,725)$ dokumentiert anhand eines Beispiels diese Wechselwirkung von Kompetenz und Performanz: Kinder, die zählen, wüssten, dass es dabei um eine bestimmte Zahlwortreihe (Zahlwörter, standardisierte Reihenfolge, jedes Wort erscheint nur ein Mal) gehe, auch wenn sie diese noch nicht fehlerlos rezitieren könnten. Für Fuson bedeutet dies, dass die Kinder etwas vom Konzept „Zählen“ wissen und es auch (evtl. nur teilweise) anwenden können, und dass dadurch etwas vom Zusammenspiel von Kompetenz und Performanz sichtbar wird (vgl. Moser Opitz 2001, 78).

\subsubsection{Kompetenz und der Erwerb von numerischen Grundlagen}

Auf der Grundlage der Annahme, dass von einem aktiv handelnden Individuum ausgegangen werden kann, wird der Erwerb der numerischen Kompetenz vor allem durch die gegenseitige Beeinflussung von Kompetenz und Performanz erklärt. Auf dieser Grundlage wird für die Erklärung der Zählentwicklung in erster Linie auf die PrinzipienNachher-Theorie zurückgegriffen. Nicht geklärt ist aber zum einen die Frage, was die gegenseitige Beeinflussung von Kompetenz und Performanz für die Entwicklung und 
Lernen ausmacht und zum zweiten, welche Schlüsse für die Arbeit von Lehrerinnen und Lehrer zu ziehen sind (vgl. Moser Opitz 2001, 80ff).

Von Interesse ist die Annahme von Siegler (1997), dass Wissen „wellenförmig“ erworben wird, indem sich das Kind langsam und von verschiedenen Seiten her einem neuen Gegenstand oder Wissensgebiet annähert. Entwicklung bedeutete nicht, dass eine bestimmte Kompetenz wie eine Stufe erreicht wird oder eben nicht erreicht wird.

„Because children's thinking is so variable, depicting cognitive development in terms of a succession of a domain-general stages or domain-specific theories is too simple. Changes in children's thinking seem typically to involve not the replacement of one way of thinking by another but rather by a gradual ebbing and flowing of multiple ways of thinking, with new approaches beeing introduced and old ones ceasing to be used as well” (Siegler 1997, 326 in: Moser Opitz 2001, 80).

Nicht allein das Erreichen eines bestimmten Levels sei wichtig bei diesem Geschehen, sondern vielmehr der Prozess, der zu diesem erweiterten Wissen führt. Das Piaget'sche Modell von Assimilation und Akkommodation mit dem ständigen Drängen zu neu zu erwerbenden Kompetenzen bilden die Basis von Siegler's Theorie. Wie Wellen, die sich überlappen - so das Bild von Siegler - führen in ihrer fortwährenden Bewegung in ihrer Gesamtheit schließlich zu neuen Konzepten.

„Use of varying approaches gives children the opportunity to observe the consequences of using each of them, and thus to learn both about the approaches and about the world. Sometimes, approaches that are superficially quite different, such as solving number conservation problems by subitizing, by counting, or by noting the type of transformations, consistently yield the same result. Such observations can lead to a more advanced understanding of quantification processes. Other times, use of multiple approaches allows children to learn when each is most effective. Thus, not only is cognitive variability a pervasive aspect of development, it also contributes to cognitive growth" (Siegler 1997, 326ff in Moser Opitz 2001, 80.).

Moser Opitz (2001, 81) kommt zum Schluss, dass dieses Modell des kognitiven Vorwärtsschreitens durch assimilative und akkommodative Prozesse noch der Ergänzung bedarf. Das Denken des Individuums könne nur dann konstruktiv eingesetzt werden, wenn ein Umfeld zur Verfügung stehe, in dem es seine Denkwerkzeuge ausprobieren und weiter entwickeln könne. Mathematisches Lernen funktioniere nur dann, wenn Situationen geschaffen werden, in denen mathematische Anforderungen auftauchen, zu de- 
ren Lösung ein Anreiz bestehe. Schon im Kindergarten, aber vor allem dann im Erstunterricht ist dies eine „Welt mit Zahlen“, in der die Kinder auf vielseitige Art und Weise Zahlen, Mengen, Zählaufgaben und weitere sinnvolle und anregende mathematischen Aufgaben und Problemen begegnen. Zu einer anregenden Umgebung, die Denkprozesse auslöst und anregt, gehört weiter der Aspekt der sozialen Beziehungen im Sinn der Möglichkeit kommunikativer Auseinandersetzung: Dies beinhaltet, dass durch Vermittlung im Sinn von anregenden Fragen, Problemstellungen und darauf folgenden Antworten, Diskussionen und Diskursen sozial vermittelte mathematische Verfahren in das konzeptuelle Wissen des Kindes integriert werden.

„Children's construction of mathematical goals and subgoals is intervowen with the socially organized activities in which they are participants; wether computing batting averages or making change for lemonade, children construct goals that are framed by cultural artefacts (...) and social interaction” (Saxe 1996, 52).

Piaget misst dem sozialen Aspekt für aktives Lernen einen hohen Stellenwert bei. In neu erschienen Texten wird insbesondere auf die Bedeutung der Auseinandersetzung mit anderen Personen hingewiesen, insbesondere im Sinne von Gruppenarbeit. In diesen Texten wird mit Nachdruck einer Vermittlung entgegnet, das Wissen ,in die Köpfe stopft“ (Piaget 1999, 179ff).

Für ein effektvolles Lern- und Entwicklungsverständnis soll nicht nur einseitig auf die eigenaktiven Lernprozesse des Kindes vertraut werden, sondern es soll auch dem Lehren in Form der Gestaltung von anregenden Lernumgebungen und kommunikativer Auseinandersetzung mehr Gewicht geben (vgl. Moser Opitz 2001, 76 - 82).

In Anlehnung an Moser Opitz $(2001,82)$ wird im Folgenden von einem Verständnis kognitiver und damit auch numerischer Entwicklung ausgegangen,

- das insbesondere bestimmt wird durch die aktive Auseinandersetzung des Individuums mit seiner Umwelt, in der es seine Denkwerkzeuge anwenden

- kanrdem soziale Beziehungen und soziale Vermittlung im Sinn von kommunikativer Auseinandersetzung eine wichtige Rolle spielen,

- $\quad$ in dem die Gestaltung der Umgebung (Kindergarten, Schule) im Sinne einer „Welt mit Zahlen“, die zur kognitiven Auseinandersetzung Anreiz bietet, beachtet werden muss,

- $\quad$ in dem sich Kompetenz und Performanz gegenseitig beeinflussen und

- das nicht durch Entwicklungsstufen bestimmt wird, sondern durch eine fortschreitende qualitative Veränderung von Wissen, die durch Beschäftigung und 
aktive Auseinandersetzung mit der Umwelt und durch das Durchschreiten unterschiedlicher Denkwege erfolgt.

\subsection{Situierte Kognition}

Im Alltag wird in der Regel davon ausgegangen, dass Wissen innerhalb einer Person angesiedelt ist, das in Form von Konzepten oder Fakten (deklarativem Wissen) sowie von strategischem und metastrategischem Prozesswissen (prozeduralem Wissen) repräsentiert ist (Siehe Seite 60, Abb. 1). Darauf kann dann im Bedarfsfall zurückgegriffen werden. Dieses Wissen, so die verbreitete Meinung, ist personenabhängig und Menschen unterscheiden sich in quantitativen und qualitativen Aspekten dieser Wissensrepräsentation. Einige Individuen verfügen über mehr Wissen, andere organisieren ihr Wissen besser. In der Psychologie wurde ebenfalls lange angenommen, dass einem Problemlöseprozess zugrunde liegende Wissen in Form von abstrakten Strukturen wie Schemata (Rumelhart, Ortony 1977) oder Skripts (Schank, Abelson 1977), Konzepten und Fakten repräsentiert wird (vgl. Stern 1998, 28ff).

Gegen Ende der 80er Jahre regte sich Widerstand gegen die Annahme, Wissen sei eine Art Substanz, die aus Begriffen bestehe, die aus allen Kontexten losgelöst sei. Der in den letzten Jahren entwickelte Ansatz der situierten Kognition (Brown, Collins und Duguid 1989; Greeno 1989; Greeno, Smith und Moore 1993; Rogoff 1990) stellt darum eine Alternative dar. Wissen, so die Theorie der situierten Kognition, kann nicht unabhängig von den Anforderungssituationen repräsentiert werden. Der Ursprung dieses Ansatzes liegt in der Tatsache, dass die Evolution Lebewesen mit den Fähigkeiten ausgestattet hat, Umweltanforderungen direkt zu bewältigen und so überleben und letztendlich die Art erhalten zu können. Diese Art der Wissensrepräsentation bezogen auf konkrete direkte Anforderungssituationen ist, so die Aussage der Theorie, jedem höheren Lebewesen eigen. Wissen, so die Sicht der situierten Kognition, ist einem Menschen nicht an sich a priori vorrätig, sondern der Mensch verfügt lediglich über Wissen zur Bewältigung einer ganz bestimmten Anforderung. „Wissen ist die Bewältigung von Anforderungen“"(Stern 1998, 29, Hervorhebung im Original).

Wissen wird in einem Lernkontext konstruiert. Als Beispiel verweise ich hier auf Kinder, die nicht in der Lage sind, einfach so bis 20 zu zählen. Sobald aber konkrete Dinge 
abzuzählen sind, schaffen dieselben Kinder das Zählen bis 20 ohne die geringste Mühe und absolut fehlerfrei (siehe Näheres Kapitel 9). Es ist notwendig, dass Wissenskonstruktion in einem Kontext stattfindet, sodass neues Wissen auf bereits Verstandenem aufbaut. Wesentlich für den Erwerb von robustem, anwendbarem Wissen ist also, dass es an realen Problemen verstanden wird. Auch hier möchte ich als Beispiel eine Interviewsequenz erwähnen: Ein Kind musste aus dem Gedächtnis die Anzahl Punkte auf zwei Würfelbilder addieren (Seite 126, Abb. 45). Das Mädchen zählte in einem ersten Durchgang die Punkte aus der Erinnerung ab und hielt in jeder Hand die jeweilige Punktzahl fest (4 und 5), zählte dann die ausgestreckten Finger ab und kam auf das richtige Resultat 9. Damit aber nicht genug: Sie schaute die noch ausgestreckten Finger an und sagte dann hocherfreut: „Vier und fünf; das ist ja neun!“ (siehe Seite 204). Um erfolgreich Aufgaben zu bewältigen oder Probleme zu lösen, müssen Wissenskonstruktionen geschaffen werden, die stabil sind und in neue Situationen transferiert werden können. Die Basis solcher Konstrukte ist die Einsicht, dass Denken und Handeln Prozesse sind, die sich aufeinander beziehen. Wissen, welches in ein Vakuum hinein konstruiert wird, ist träge, bleibt nicht haften und kann darum nur schwer in wirklichen Problemstellungen angewandt werden (vgl. Stern 1998, 29 und auch Brown, Collins und Duguid 1989; Spiro, e.a. 1991; Winn 2001).

Dieser Ansatz der situierten Kognition hat Auswirkungen auf das Lernen: Wissen kann nicht einfach nur von einer Person zur anderen weitergereicht werden, sondern entsteht aus einem aktiven Konstruktionsprozess des Lernenden. Situiertes Lernen gilt als ein Prozess, in dem personeninterne Faktoren mit personenexternen, situativen Komponenten in Wechselbeziehung stehen. Der Situationsbegriff meint hier nicht nur materielle Aspekte, sondern auch die soziale Umwelt des Lernenden. Daher spielen beim situierten Lernen auch die Interaktionen zwischen Menschen sowie die historischen und kulturellen Kontexte, die in ihr Handeln und Denken eingebettet sind, eine besondere Rolle.

Wirkliches Verstehen entwickelt der Lernende erst, indem er Gelerntes kontinuierlich in Situationen anwendet. Diese Erkenntnis kann auf alle Arten von Wissen angewendet werden. Das Individuum versucht mit den wesentlichen Teilen des Wissens die Welt zu erfassen und darzustellen und diese Wissenskomponenten sind somit ein Produkt der Aktivitäten und Situationen, innerhalb derer sie entstanden sind. Deklaratives Wissen kann in gewisser Weise mit einem Werkzeugsatz verglichen werden. Genau wie Wissen können Werkzeuge nur durch Benutzung voll verstanden werden. Es ist durchaus möglich, ein Werkzeug zu kennen und trotzdem nicht in der Lage zu sein, es auch zu benut- 
zen. Menschen, die Werkzeuge kontinuierlich benutzen, bauen ein umfassendes Verständnis für die Welt, in der sie das Werkzeug nutzen und für das Werkzeug selbst auf. Das Verstehen der Welt und des Werkzeuges verändert sich ständig durch deren Zusammenspiel. Lernen ist ein kontinuierlicher lebenslanger Prozess, der aus dem Handeln in Situationen entsteht.

$\mathrm{Zu}$ lernen, wie ein Werkzeug zu benutzen ist, beinhaltet viel mehr, als nur ein Bündel von Regeln. Die Möglichkeiten und Rahmenbedingungen für die Benutzung hängen direkt mit dem Kontext der Aktivitäten, innerhalb derer eine Gemeinschaft dieses Werkzeug benutzt, zusammen - determiniert durch die Art und Weise, in der die Mitglieder der jeweiligen Gesellschaft die Welt sehen. Die Gesellschaft und deren Sichtweise also legen genauso wie das Werkzeug selbst fest, wie ein Werkzeug benutzt wird. Damit ist es nicht möglich, ein Werkzeug angemessen zu benutzen, wenn man die Gesellschaft oder die Kultur, in deren Rahmen es benutzt wird, nicht verstanden hat. Um also Werkzeuge/Wissen so zu benutzen wie Praktiker das tun, muss der Lernende in diese Gesellschaft und deren Kultur eintreten. Damit ist Lernen ein Prozess der Kultivierung.

Lernende müssen Wissen im Rahmen authentischer Aktivitäten erlernen, unterstützt von Lehrern, die wie Praktiker dieses Wissen durch das Ringen mit Problemen der Welt anwenden und so weitergeben (vgl. Brown, Collins, Duguid 1992).

Lernen ist also als aktiver Konstruktionsprozess des Lernenden zu konzipieren. Wissen wird nicht einfach transportiert, sondern vielmehr individuell konstruiert.

Der Ansatz der situierten Kognition fordert, dass bei der Gestaltung der Lernumgebung die grundsätzliche Situiertheit des Lernens berücksichtigt wird. Eine Konsequenz aus dieser Forderung ist, dass das Lernen nicht mehr generell als Transport eines abstrakten Wissensstandes aufgefasst wird, da nur selten dieser Vermittlungsstil mit der späteren Möglichkeit der Wissensnutzung korrespondiert. Obwohl „Lernen“ von theoretischem Wissen auch heißt, sich von spezifischen Situationen lösen zu können, ist mit einem Wissenstransfer im Allgemeinen nur dann zu rechnen, wenn der instruktionale und der Anwendungskontext einander ähnlich sind. Das erworbene Wissen kann dann auch außerhalb der Lernsituation verwendet und eingesetzt werden, es findet also erfolgreicher Transfer statt - dies in Übereinstimmung mit den Aussagen von Lave/Wenger (1991), wonach Wissenstransfer nicht die Regel ist, sondern immer einen hohen Energieeinsatz aller Beteiligten, insbesondere der Lehrperson voraussetzt.

Fasst man die Ansätze situierten Lernens zusammen, lassen sich folgende Merkmale festhalten: 
- Wissen ist immer situiert, daher ist auch Lernen immer situiert.

- Wissen wird durch das wahrnehmende Subjekt konstruiert.

- Besonders wesentlich ist das in einer Gesellschaft geteilte Wissen; Lernen ist daher zunehmende Teilhabe an einer Expertengemeinde.

- Situiertes Wissen wird unter dem Anwendungsaspekt und damit unter dem Gesichtspunkt seiner Authentizität analysiert.

Alle Instruktionsansätze situierten Lernens schlagen Lernen durch aktives Lösen von komplexen Problemen vor. Dadurch soll die Anwendungsqualität des erworbenen bzw. konstruierten Wissens erhöht werden.

Die Gemeinsamkeiten dieser Ansätze lassen sich in folgenden grundlegenden Forderungen für die Gestaltung von Lernumgebungen zusammenfassen:

- Komplexe Ausgangsprobleme

- Authentizität und Situiertheit

- Multiple Perspektiven

- Artikulation und Reflexion

- Lernen im sozialen Austausch

Die Grundkonzeptionen der Modelle der situierten Kognition erinnern an einige bereits bekannte Konzepte wie z.B. Gruppenarbeit oder Fallstudien. Diese fanden ihre Legitimation jedoch weniger durch Befunde der Kognitionspsychologie als durch systematische Erfahrungen in der Praxis. Eine offene Frage ist nach H. M. Niegemann (1995), ob sich diese Ansätze in ihrer breiten Anwendung als effektiv erweisen werden, d.h. unter dem Aspekt der Kosten und der Lernzeit vergleichbare Ergebnisse erzielen wie die herkömmlichen Strategien. Voraussetzungen sind oft unabdingbar in dem Sinne, dass sich der Lernende auf fortgeschrittenem Wissensniveau befindet und neben inhaltlichem Wissen auch Problemlöse- und Selbststeuerungsfertigkeiten entwickeln soll. Der Lernende hat keine oder kaum Erfahrung mit den neuen Inhalten und soll zunächst lernen, wie das angeeignete Wissen mit realen Erfordernissen zusammenhängt. Nach H. Mandl (1992) lassen sich drei Problemkreise formulieren:

\section{Empirische Probleme:}

Die empirische Befundlage ist bisher sehr schmal und Instrumente der Evaluation mit empirischer Absicherung liegen zurzeit noch nicht vor. 
Theoretische Probleme:

- Nach der Position des radikalen Konstruktivismus ist Wissensvermittlung nicht möglich, erst durch die gemäßigtere Form wird die Annahme einer äußeren Einflussnahme integriert. Problematisch bleibt jedoch die mögliche Beliebigkeit bei der Gestaltung von Lernumgebungen.

- Das Postulat der Autonomie und Selbstverantwortung bringt die Gefahr mit sich, bestehende Macht- und Hierarchiestrukturen zu übersehen und das Individuum alleine für seine Situation verantwortlich zu machen.

Praktische Probleme:

- Im Vergleich zu den traditionellen Ansätzen ist diese Gestaltung von Lernumgebungen zeitintensiv und verlangt vom Lernenden eine höhere „Investition“ an Zeit und Anstrengung. Fehlende Unterstützung kann überfordern und die Motivation nachteilig beeinflussen. Dieses Gleichgewicht zwischen Freiräumen für konstruierende Aktivitäten und gezielter Anleitung und Unterstützung ist schwer umzusetzen.

- Es besteht die Gefahr der Reduktion sachlogisch geordneter und systematischer Kenntnisse sowie die Gefahr der Vernachlässigung der Entwicklung automatisierter Routinefertigkeiten.

\subsection{Mentale Modelle}

Seit den 80er Jahren hat die psychologische Theorie mentaler Modelle mehr und mehr Anhänger in der Kognitionsforschung gewonnen. Modelle sind in der Regel vereinfachte und auf wesentliche Komponenten reduzierte Abbilder der Realität. Unter mentalen Modellen versteht man individuelle Denkmodelle, die das Verständnis eines Sachverhaltes prägen, ,mit deren Hilfe wir planen und entscheiden, vorausschauen und erklären, kurz: mit deren Hilfe wir denken“" (Hasebrook 1995, 124). Beispiele für mentale Modelle sind die Darwinsche Evolutionstheorie, die Vorstellung vom Herz als einer Pumpe oder der Anordnung der natürlichen Zahlen auf dem Rechenstrich (Zahlenstrahl ohne Skalierung). Diese mentalen Modelle können für bestimmte Aspekte eines Gegenstandsbereiches hinreichend sein, für andere jedoch nicht. Die Assoziation eines Strom führenden Kabels mit einem Wasserschlauch macht beispielsweise viele Aspekte ver- 
ständlich (z.B. Widerstand), versagt jedoch bei anderen, z.B. bei einer Unterbrechung des Kabels (Hasebrook 1995, 124).

Mentale Modelle sind dynamisch, d.h. sie werden mit zunehmendem Verständnis eines Sachverhaltes, eines Objektes oder eines Prozesses elaboriert und angepasst.

Über die Repräsentation mentaler Modelle gibt es unterschiedliche Vorstellungen. Weidenmann (1997, 73) beschreibt mentale Modelle als Mischformen von bildhaften und propositionalen Repräsentationen. Als Proposition wird die kleinste Bedeutungseinheit bezeichnet, die als selbständige Behauptung stehen kann, also die kleinste Einheit, die sinnvoll als wahr oder falsch beurteilt werden kann. Beispielsweise lässt sich die Bedeutung des Satzes „Anna konstruiert mit Hans, der zu Besuch aus einer anderen Klasse im Klassenzimmer weilt, einen schönen Faltschmetterling. “ in die folgenden einfachen Behauptungen umformulieren:

1. „Anna konstruiert mit Hans einen Faltschmetterling.“

2. „Der Faltschmetterling ist schön.“

3. „Hans ist zu Besuch aus einer anderen Klasse im Klassenzimmer.“

Falls eine dieser Behauptungen falsch wäre, wäre auch der komplexere Satz falsch. Diese Behauptungen entsprechen den Propositionen, die der Bedeutung des Satzes zugrunde liegen. Jede einzelne ist Ausdruck einer elementaren Bedeutungseinheit.

Eine propositionale Repräsentation besteht somit aus diskreten Sinneseinheiten, die ihrerseits ähnlich wie Sätze und Phrasen der natürlichen Sprache nach bestimmten syntaktischen Regeln aus einfachen Symbolen zusammengesetzt sind und insofern eine bestimmte Phrasenstruktur besitzen. Die propositionale Repräsentation kann als innere mentale Sprache angesehen werden, die den gemeinten Sachverhalt beschreibt. Sie basiert auf der Kombination dieser internen Symbole und ist insofern als digitale Repräsentation anzusehen, dass trotz einer großen Zahl von verfügbaren Regeln und möglichen Verknüpfungen der Zeichenvorrat zwar sehr groß aber letztendlich doch begrenzt ist.

Dagegen geht Edelmann (1996, 252f) davon aus, dass mentale Modelle sowohl sprachliche als auch bildhafte und handlungsbezogene Komponenten aufweisen und ganzheitlich-analog orientiert sind. Er beschreibt mentale Modelle als „,subjektive Wissensgefüge mit funktionalem Charakter", die der inneren Simulation äußerer Vorgänge dienen. In diesem Sinne argumentieren Greeno et al. (1993), für die mentale Modelle handlungsrelevante Merkmale der Situation gespeichert haben, die helfen, die in einer bestimmten Situation gestellte Anforderung zu bewältigen. Im Ansatz der situierten Kog- 
nition nach Greeno et al. (1993) wird unter einem mentalen Modell die Repräsentation einer ganz spezifischen Anforderungssituation verstanden. „Mentale Modelle sind nicht als Abbilder der Realität, sondern als auf das Handlungsziel bezogene Ausschnitte aus der Realität zu verstehen“ (Stern 1998, 30, Hervorhebung im Original). Wenn ein Einheimischer einem Ortsunkundigem den kürzesten Weg zum Opernhaus beschreiben will, muss er sich ein mentales Modell des Weges konstruieren, das aus einem imaginären Gang durch die Stadt besteht. Dieses Modell wird aus Wissen aufgebaut, das in früheren Anforderungssituationen zur Konstruktion von mentalen Modellen herangezogen wurde. Die konstruierten mentalen Modelle können in ihrer Effizienz in Bezug auf die Bewältigung der Anforderung variieren. Entscheidend für die Effizienz, mit der die Anforderungssituation „Ermittlung des kürzesten Weges zum Opernhaus“ bewältigt wird, ist, nach welchem Kriterium der Begriff „kurz“ abstrahiert wird. Bedeutet in diesem Zusammenhang „kurz“ den effektiv kürzesten oder doch eher den schnellsten Weg. Es wäre ineffizient, wenn man von jeder Strasse ein detailliertes Bild erzeugen würde, in der Verkehrsdichte, Anzahl Ampel, vor denen man mit großer Wahrscheinlichkeit warten müsste, topographische Situationen u.a. vorkommen. Ineffizient wäre es auch, wenn ein detailliertes mentales Modell von Strassen, die nicht direkt zur Oper führen, konstruiert würde. So kann das in früheren Anforderungssituationen erworbene Wissen als Ortskundiger den imaginären Gang durch die meisten Strasse ersparen und den optimalen Weg beschreiben.

„Die Bewältigung von Anforderungen besteht also in der sukzessiven Konstruktion von mentalen Modellen. Am Ende eines erfolgreichen Problemlöseprozesses wird ein auf die Grundstrukturen reduziertes mentales Modell konstruiert, das zur Ausführung der aktionalen Voraussetzungen führt - in vielen Fällen lediglich eine schriftlich oder mündlich geäußerte sprachliche Antwort“ (Stern 1998, 31).

Demnach konstruiert ein Leser beim Verstehen eines Textes oder eines mathematischen Problems sowohl eine so genannte propositionale Repräsentation des Textes als auch auf der Basis dieser Repräsentation - ein mentales Modell des dargestellten Sachverhaltes. Es wird davon ausgegangen, dass propositionale Repräsentationen und mentale Modelle auf unterschiedlichen, jedoch einander ergänzenden Repräsentationsprinzipien beruhen.

Bei einem mentalen Modell handelt es sich um ein internes, hypothetisches (Quasi-) Objekt, das in einer Struktur- oder Funktionsanalogie zu dem dargestellten Gegenstand steht und diesen aufgrund dieser Analogie repräsentiert. Bei einer Strukturanalogie - 
wie z.B. zwischen dem Bohrschen Atommodell und dem Sonnensystem - besteht Übereinstimmung zwischen der Struktur der Systeme (Planeten bzw. Elektronen „umkreisen“ Sonne bzw. Atomkern), wobei von der konkreten stofflichen Realisierung der Systeme abstrahiert wird. Bei einer Funktionsanalogie - wie z.B. zwischen einem elektrischen Schwingkreis und einem mechanischen Pendel - besteht Übereinstimmung in der Funktion der Systeme (harmonische Oszillatoren), wobei sowohl von der konkreten stofflichen Realisierung als auch von der Struktur der Systeme abstrahiert wird. Ein mentales Modell wird so nicht zwangsläufig eine bildhafte Darstellung des jeweiligen Gegenstandes sein.

Propositionale Repräsentationen und mentale Modelle sind komplementäre, einander ergänzende Formen der mentalen Repräsentation, zwischen denen ein ständiges Wechselspiel stattfindet: Propositionale Repräsentationen dienen als Basis für die Konstruktion eines mentalen Modells, und die Ergebnisse der Modellanalyse werden wiederum in propositionaler Form fixiert und stehen somit als neue Daten zur Konstruktion neuer mentaler Modelle zur Verfügung.

Das Konzept des mentalen Modells - auch als Mittel des Erkenntnisgewinns - hat sich in den letzten Jahren zu einem Forschungsschwerpunkt der Kognitionswissenschaften entwickelt.

Aus Untersuchungen, bei denen u.a. mentale Modelle verschiedener Personen mit einem optimalen konzeptuellen Modell, das heißt einem Modell, das einen Gegenstand optimal repräsentiert, verglichen werden, kristallisieren sich folgende Merkmale mentaler Modelle heraus (Dutke 1994; Weidenmann 1994):

- Mentale Modelle sind sowohl reduzierte als auch elaborierte Abbildungen. Nicht alle Merkmale und Relationen des Originals werden abgebildet. Die Abbildung hängt von den Vorkenntnissen und Intentionen der betreffenden Person ab. Zusätzliche Merkmale, die in mentale Modelle eingehen, können aus bereits vorhandenem Wissen stammen.

- Mentale Modelle haben verschiedene Funktionen. Zum einen ermöglichen sie das Verstehen von Sachverhalten, zum anderen ermöglichen sie mit Hilfe der kognitiven Simulation eine Vorhersage über zukünftige Systemzustände.

- Als nützlich erwiesene mentale Modelle sind manchmal schwer zu ändern. Die Änderung eines allgemeineren Modells zieht die notwendige Angleichung der von ihm abhängigen spezielleren Modelle nach sich. 
- Mentale Modelle sind unwissenschaftlich. Die Personen halten an irrationalen Verhaltensweisen fest, selbst wenn sie wissen, dass diese wirkungslos sind, nur weil diese einen geringen physischen und geistigen Aufwand verlangen.

- Mentale Modelle sind simulationsfähig. Durch gedankliche Simulation kann Wissen auf neue Problemstellungen und Anwendungsgebiete übertragen werden.

- Mentale Modelle erzeugen Abbildungen, die unvollständig, unscharf oder widersprüchlich sein können.

- Mentale Modelle sind instabil. Die Personen vergessen z.B. Details eines Gerätes, das sie bedienen, vor allem wenn diese Details oder das ganze System eine Zeitlang nicht benutzt wurde.

Die Forschung beschäftigt sich bisher fast ausschließlich mit der Frage, ob und unter welchen Bedingungen ein Sachverhalt bildhaft darzustellen ist, um so dessen Verständnis zu erleichtern bzw. um ein wirksames Lernen zu ermöglichen. Bisher wurden allerdings noch keine Aussagen dazu gemacht, wie dieser Sachverhalt visualisiert werden sollte bzw. welche von verschiedenen Möglichkeiten der Visualisierung am geeignetsten ist.

Im Hinblick auf die Entwicklung eines Systems zur Wissensvermittlung lassen sich jedoch drei wesentliche Eigenschaften mentaler Modelle herausstellen:

- Mentale Modelle entstehen aus Erfahrungen, die während der Interaktion mit der Umwelt im Laufe des Lebens gewonnen werden.

- Der Aufbau mentaler Modelle kann durch die Berücksichtigung bereits vorhandener mentaler Modelle (Erfahrungen einer Person) erleichtert

- Mendente Modelle beschreiben die Welt in Begriffen, die dem subjektiven Denken einer Person entsprechen.

Während das Interesse der Kognitionswissenschaftler darin liegt, die Prozesse zu beschreiben und plausibel zu machen, die bei der Wissensbildung und Wissensrepräsentation eine Rolle spielen, entwickelt die Pädagogik Modelle und Methoden, die sich in erster Linie damit befassen, wie man Lernen anregen und unterstützen kann. 
Um den Kindern das Lernen zu ermöglichen und zu erleichtern, werden vielfach Veranschauungsmittel und Anschauungsmittel ${ }^{10}$ bemüht. Im Zuge des konstruktivistischen Lehransatzes findet momentan auf breiter Front ein Paradigmenwechsel ${ }^{11}$ im Sinne einer Verschiebung „,von Werkzeugen des Lehrens zu Werkzeugen des Lernens“ (Krauthausen \& Scherer 2001, 212) statt. Das bedeutet, dass Anschauungsmittel Werkzeuge des Lernens für alle Kinder sein müssen; denn „Anschauung ist nicht eine Konzession an angeblich theoretisch schwache Schüler, sondern fundamental für Erkenntnisprozesse überhaupt“ (Winter 1996, 9 in Krauthausen \& Scherer 2001, 213). Wesentlich ist, dass sich die Lernenden das Wahrnehmungsobjekt in Form eines aktiven kognitiven Vorganges aneignen. „Es besteht keine direkte, zwingende Verbindung zwischen Wahrnehmungsobjekten oder Veranschaulichungen und dem Denken des Lernenden“ (Krauthausen \& Scherer 2001, 213). Ziel des Einsatzes von Anschauungsmitteln ist u.a. der Aufbau von mentalen Modellen. Wie oben dargelegt, sind die daraus entstehenden mentalen Modelle nicht bloßes Abbild der Sinneswahrnehmung, sondern sie sind ganz individuell geprägt durch Gewohnheiten, Vorlieben, Vorerfahrungen und Denkstrukturen. Es ergeben sich so ganz verschiedene Vorstellungsbilder, auch wenn die Kinder in der Schule oder im Kindergarten mit den gleichen Arbeitsmitteln und Veranschaulichungen arbeiten. Im Weiteren kann nicht ohne weiteres geschlossen werden, dass für Erwachsene strukturgleiche Anschauungsmittel ein einziges mentales Modell erzeugen. So ,ist ein Addieren oder ein Subtrahieren mit Perlen, mit Steckwürfeln, mit Fingern oder mit Rechengeld jeweils ein Operieren in einer anderen ,Mikrowelt', zwischen denen nicht einfach eine abstrakte (mathematische) Beziehung besteht" (Radatz 1991,

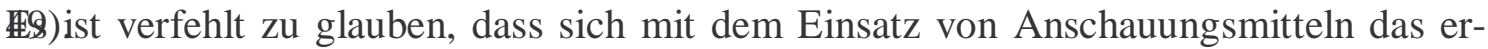
wünschte Verstehen eines mathematischen Sachverhaltes automatisch einstellt. Die Konstruktion des zum vollständigen Verstehen und Durchdringen des fraglichen Sachverhaltes notwendigen mentalen Modells ist, wie wir gesehen haben, sehr individuell geprägt und erfordert eine eigene intensive Auseinandersetzung mit dem mathematischen Sachverhalt, um es mit dem dargebotenen Arbeitsmaterial in Deckung zu bringen. Dieser konstruktive Akt des Lernenden ist sehr anspruchsvoll und nicht immer gelingt

\footnotetext{
${ }^{10}$ Es ist zwischen Veranschauungsmitteln und Anschauungsmitteln zu unterscheiden. Erstere werden in erster Linie von der Lehrperson eingesetzt, um mathematische Ideen oder Konzepte zu illustrieren. Als Werkzeug der Lehrperson unterliegen sie dem didaktischen passivistischen Grundverständnis, dass Wissen vom Lehrenden zum Lernenden übermittelt werden könne. Der Begriff der Anschauungsmittel entspricht eher dem aktivistischen Lernverständnis: Das Arbeitsmittel wird in die Hände der Lernenden gelegt, als Werkzeug ihres eigenen Mathematiktreibens (vgl. Krauthausen \& Scherer 2001, 212).

${ }^{11}$ Paradigma: ,,allgemein anerkannte wissenschaftliche Leistungen, die für eine gewisse Zeit einer Gemeinschaft von Fachleuten massgebende Probleme und Lösungen liefern“ (Kuhn 1989,10).
} 
es allen Kindern, die zugrunde liegende mathematische Struktur zu erkennen. „Bei den zahlreichen Ikonisierungen im arithmetischen Anfangsunterricht wird offensichtlich als selbstverständlich vorausgesetzt, dass die zum Verstehen der graphischen Darstellung notwendigen geometrischen Kenntnisse und Fähigkeiten (geometrische Ordnungsbeziehungen, geometrische Qualitätsbegriffe, Eigenschaften ebener Figuren und Anordnungen, räumliches Vorstellenkönnen u.v.a.m.) bei allen Schulanfängern und Grundschülern gleichermaßen vorhanden sind. Das ist ein verhängnisvoller Irrtum“ (Radatz 1993, $50)$.

„Es gibt keine, automatische’ Verinnerlichung mathematischer Ideen, Strukturen oder Begriffe, nur weil mit Arbeitsmitteln konkret gehandelt wird“ (Krauthausen \& Scherer 2001, 216). Mit anderen Worten: Die Bildung von mentalen Modellen ist nicht nur vom konkreten Anschauungsmittel abhängig, sondern es braucht einen ,selbständigen konstruktiven Akt“" (ebd.). Erst auf dieser von Eigeninitiative geprägten Basis entsteht ein tragfähiges mentales Modell, ,an denen die arithmetischen Operationen in der Vorstellung ausgeführt und die Rechenergebnisse ,abgelesen’ werden können“ (Gerster 1994, 41; Hervorh. im Orig.). Ein Beispiel möge illustrieren, wie vielfältig die Anforderungen sind: „Die Hunderter-Tafel verkörpert in prägnanter Weise die dezimale Struktur unseres Zahlensystems und hilft Einsichten zu gewinnen in Analogien, die auf dieser Struktur beruhen. [...] Das konkrete Modell der Hunderter-Tafel muss zu einem mentalen Modell, gleichsam einer ,Hunderter-Tafel im Kopf’ werden. Diese darf man sich nun nicht als eine Kopie des konkreten Materials vorstellen, sie ist vielmehr das verinnerlichte Wissen über die strukturellen Eigenschaften der Zahlen bis 100, insbesondere das Wissen über Stellenwerte, Nachbarschaftsbeziehungen, Zehnernachbarschaften und über Analogien“" (Radatz et al. 1998, 35).

Die ersten mentalen Modelle werden im Kindergartenalter ausgebildet ${ }^{12}$ und dann vor allem im 1. Schuljahr während der Arbeit im Zahlenraum bis 20. Die Lehrkraft übernimmt eine große und verantwortungsvolle Aufgabe, tragfähige und robuste mentale Modelle entstehen zu lassen. „Aufgrund der fundamentalen Bedeutung des mentalen Vorstellens und Operierens für spätere Lernprozesse liegt hier eine ganz entscheidende Stelle der Unterrichtspraxis vor. Eine verfrühte Abkehr von anschaulichen Darstellungen, bevor wirklich tragfähige mentale Bilder vom Kind konstruiert und genutzt werden können, kann als der Kardinalfehler des Anfangsunterrichts bezeichnet werden“ (Kraut-

\footnotetext{
${ }^{12}$ In einigen Kantonen der Schweiz insbesondere beim Erarbeiten der Zahlen bis 10.
} 
hausen \& Scherer 2001, 217; Hervorh. im Orig.). ${ }^{13}$ Im Abschnitt 10.2 wird dargelegt, wie bereits im Kindergarten der Aufbau adäquater mentaler Modelle gelegt werden kann.

${ }^{13}$ Bei vielen Bildungsexpertinnen und -experten herrscht die Meinung vor, dass gerade Grundschulkinder mathematische Sachverhalte grundsätzlich nur ausgehend von konkreten Handlungen lernen können. Diese Sichtweise ist aber zu eng und entspricht nicht dem Stand der Forschung. Man kann gerade die weniger leistungsstarken Kinder am besten fördern, wenn man ihnen gezielt dabei hilft, die in den realen Situationen enthaltenen Beziehungen, Muster und Strukturen zu erkennen, die sie - anders als die leistungsstärkeren Kinder - nicht selber finden (Hasemann 2003a). 


\section{Untersuchungsinstrumentarium}

\subsection{Osnabrücker Test zur Zahlbegriffsentwicklung}

Der „Osnabrücker Test zur Zahlbegriffsentwicklung“ (OTZ) ist wie schon erwähnt ein Test, mit dem die frühe mathematische Kompetenz von ca. 4- bis ca. 7-jährigen Kindern in ihrer ganzen Breite erhoben werden kann. Er ist in Anlehnung u.a. an Piaget, Fuson und Gelman \& Gallistel sowie unter Beteiligung von Mathematikdidaktikern wie A. Treffers von der Universität Utrecht entwickelt worden und wurde 1994 in Holland mit über 800 Kindern erprobt. Eingesetzt wird er in Holland vor allem mit dem Ziel, ggf. vorhandene Defizite der Kinder in gewissen Teilbereichen der mathematischen Kompetenz frühzeitig zu erkennen und die Kinder daraufhin gezielt fördern zu können. Bei der Bearbeitung des Tests im Rahmen von halbstandardisierten Interviews (siehe Kapitel 6.2) löst je ein Kind im Beisein eines Testers die Aufgaben anhand vorgelegter Bilder, mit Material oder verbal. Da die Ergebnisse halbstandardisiert sind, lässt sich für jedes Kind eine Einordnung seiner frühen mathematischen Kompetenz relativ zu der seiner Altersgenossen vornehmen, und es lässt sich feststellen, in welchen Bereichen gegebenenfalls besondere Stärken oder Defizite vorliegen (vgl. Hasemann 1998, 264).

Im Zusammenhang mit früher mathematischer Entwicklung werden nebst den Zählkompetenzen oft auch Piaget's Operationen Seriation, Klassifikation, Korrespondenz und Invarianz genannt, wobei sich allerdings diese Operationen bei Piaget ursprünglich auf das logische Denken bezogen. Diese Operationen werden in Zählkontexten verwendet wegen den wechselseitigen Beziehungen zwischen den verschiedenen Zählkompetenzen und den Operationen Seriation, Klassifikation, Korrespondenz und Invarianz. Die Beziehungen zwischen Seriation und Klassifikation einerseits und Ordinal- und Kardinalzahl andererseits sind in der Literatur oft beschrieben worden (vgl. Brainerd 1979; Sophian 1992; Moser Opitz 2001). Durch Klassifikation kommt man zur Kardinalzahl ${ }^{14}$ und durch Seriation zur Ordinalzahl ${ }^{15}$. Bei Piaget's Operation der Klassifikation ist die Eins-Zu-eins-Zuordnung der allerwichtigste Aspekt. Für das korrekte Zählen ist dieser Aspekt unabdingbar: ein Zahlwort für genau ein Element. Um Mengen von Elementen abzuzählen oder Fragen nach der Rangordnung von Elementen richtig beantworten zu

\footnotetext{
${ }^{14}$ Z.B. bezeichnet das Kardinalzahlwort ,acht“ eine bestimmte Anzahl Elemente in einer Menge.

${ }^{15}$ Z.B. bezeichnet das Ordnungszahlwort „,das achte“ ein bestimmtes Element in einer geordneten Menge.
} 
können, muss man die Eins-zu-eins-Zuordnung fehlerfrei anwenden können. Für die Entwicklung des frühen Zahlbegriffs ist die Invarianz der Zahl die wichtigste Invarianz überhaupt. In einer Aufgabe zur Invarianz der Zahl muss das Kind in der Lage sein zu verstehen, dass sich Aussagen wie „mehr als“ oder „weniger als“ auf die Anzahl der Elemente einer Menge bezieht und nicht auf die räumliche Ausdehnung diese Elemente (vgl. van Luit, van de Rijt, Hasemann 2000).

Um die Entwicklung des frühen Zahlbegriffs von vier- bis siebenjährigen Kindern umfassend darstellen zu können, erkannten die Autoren aus der Analyse früherer Studien (Van de Rijt 1996, Van de Rijt, Van Luit \& Pennings 1994), dass acht Komponenten des Wissens über numerische und nicht-numerische Quantitäten genügten, um diesen frühen Zahlbegriff zu begründen.

Es sind dies die folgenden acht Komponenten:

1. Vergleichen

2. Klassifizieren

3. Eins-zu-eins-Zuordnung

4. Nach Reihenfolgen ordnen

5. Zahlwörter gebrauchen

6. Strukturiertes Zählen

7. Resultatives Zählen

8. Allgemeines Zahlenwissen

Diese Komponenten ${ }^{16}$ werden im Folgenden mit je einer Beispielaufgabe näher erläutert:

1. Vergleichen: Die Kinder sollen zeigen, dass sie den Gebrauch von Vergleichsbegriffen wie größer/kleiner, höher/tiefer oder die meisten/die wenigsten beherrschen, die Voraussetzung für die Bildung mathematischer Ordnungsbegriffe und -relationen sind. Äquivalente oder nicht äquivalente Situationen werden von vierjährigen Kindern im Allgemeinen bewältigt, indem sie vergleichen und Begriffe wie niedrig, niedriger, am niedrigsten; mehr, weniger, am wenigsten; dicker, dick und dünn gebrauchen (Gelman \& Baillargeon 1983). Zum Nachweis dieser Kenntnis werden nicht äquivalente Kardinalsituationen (mehr/weniger), Ordinalsituationen (Reihen-

\footnotetext{
${ }^{16}$ Es muss noch darauf hingewiesen werden, dass die Aufgaben dieser Komponenten unter einem psychologisch theoriegeleiteten und nicht unter einem lernbiographisch und lebensweltbezogenen Ansatz entstanden sind.
} 
folgen) oder Maßsituationen (höher/tiefer) verwendet. Dazu eine Beispielaufgabe: „Hier siehst du Gebäude. Zeige auf das niedrigste Gebäude.“ Das Kind muss die Kenntnis der Begriffe immer durch Zeigen nachweisen.

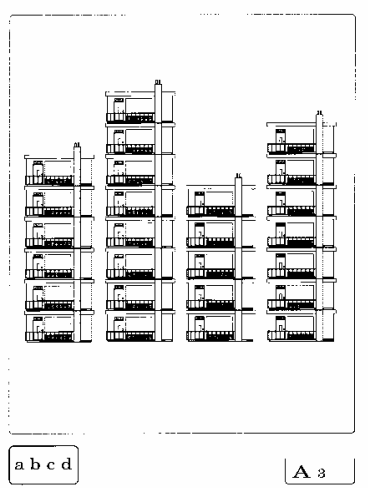

Abb. 2 Beispielaufgabe Vergleichen

2. Klassifizieren (vgl. Piaget 1965): Hier sollen die Kinder zeigen, dass sie in der Lage sind, Gegenstände nach einer Merkmalsausprägung oder auch nach mehreren Merkmalsausprägungen (im Sinne von Übereinstimmungen oder Unterschieden) zu einer Klasse zusammenzufassen. Beispielaufgabe: „Hier siehst du einen Apfel mit Stiel, ohne Blatt und mit einem Würmchen, das aus dem Apfel herauskommt. Zeige auf alle Äpfel, die genau gleich aussehen. “

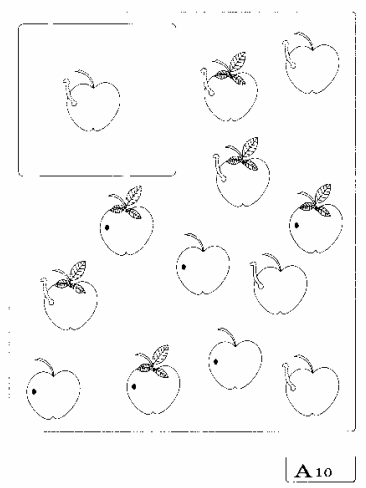

Abb. 3 Beispielaufgabe Klassifizieren

3. Eins-zu-eins-Zuordnung (vgl. Piaget 1965): Dieses fundamentale Zuordnungsprinzip ist für die Zahlbegriffsbildung unentbehrlich. Hier wird die Einsicht des Kindes in die Eins-zu-eins-Zuordnung bei zwei Mengen von Gegenständen getestet. Durch die Eins-zu-eins-Zuordnung kann ein Vergleich der Mächtigkeit von Mengen zwischen verschiedenen Objekten auch ohne Zählen hergestellt werden. Beispielaufga- 
be: [Das Kind hat fünfzehn Holzwürfel.] Der Versuchsleiter fragt: „Kannst du genau so viele Holzwürfel auf den Tisch legen wie hier gewürfelt wurde?" Zum Nachweis der Einsicht in die Eins-zu-eins-Zuordnung sind offene oder versteckte Handlungen (wie das Bewegen von Holzwürfeln, Zeichnen von Linien, mit den Fingern zeigen o.ä.) notwendig.

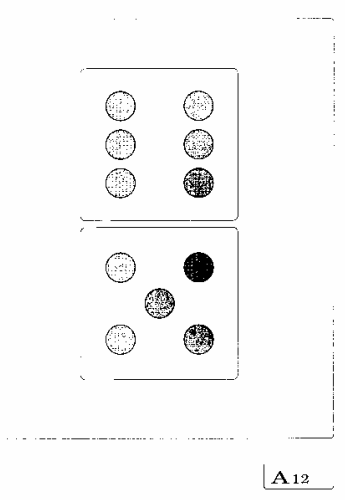

Abb. 4 Beispielaufgabe Eins-zu-eins-Zuordnung

4. Nach Reihenfolgen ordnen (vgl. Piaget 1965): Die Kinder zeigen, dass sie das Umgehen mit einzelnen und geordneten Dingen beherrschen. Es wird hier festgestellt, ob die Kinder in der Lage sind, eine korrekte oder inkorrekte Anordnung zu erkennen. Dabei werden folgende Begriffe benutzt: Von dick nach dünn, von eng nach breit, von hell nach dunkel, von hoch nach niedrig. Im Weiteren müssen die Kinder Reihenfolgen selber herstellen, indem sie Linien zeichnen. Beispielaufgabe: „Hier siehst du Hunde. Jeder Hund soll einen Stock bekommen. Ein großer Hund bekommt einen großen Stock und ein kleiner Hund bekommt einen kleinen Stock. Zeichne Linien von den Hunden zu den Stöcken, die sie bekommen. “

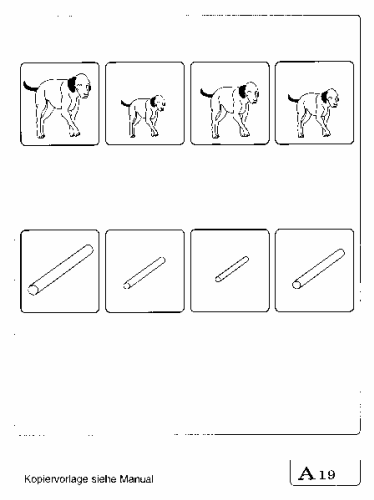

Abb. 5 Beispielaufgabe Nach Reihenfolgen ordnen 
5. Zahlwörter gebrauchen: Bei diesen Aufgaben geht es um den Gebrauch der Zahlwortreihe bis zwanzig, wobei die Kinder vorwärts zählen, rückwärts zählen und weiterzählen müssen. Es wird ferner überprüft, ob die Kinder die Kardinal- und Ordinalzahlen bis zwanzig korrekt verwenden können. Beispielaufgabe: „Zähle bis vierzehn und überspringe jedes Mal eine Zahl: zwei, vier, sechs, ... “

6. Strukturiertes Zählen: Die Mächtigkeit einer Menge wird im Allgemeinen durch Abzählen bestimmt, wobei die letztgenannte Zählzahl die Kardinalzahl darstellt, die die gesuchte Mächtigkeit der Menge angibt. Korrektes Zählen bedingt die Kompetenz einer einwandfreien Eins-zu-eins-Zuordnung. Diese Aufgaben beziehen sich nun auf das Zählen von Gegenständen in geordneter und ungeordneter Anordnung mit Zeigen. Das Kind darf alle ihm zur Verfügung stehenden Hilfsmittel gebrauchen; insbesondere darf es die Würfel auch berühren und herumschieben. Beispielaufgabe: [Der Versuchsleiter legt zwanzig Holzwürfel ungeordnet auf den Tisch.] „Zähle diese Holzwürfel.“

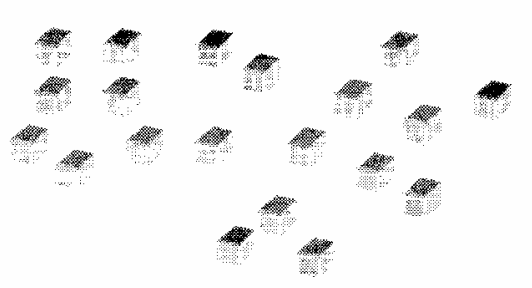

Abb. 6 Beispielaufgabe Strukturiertes Zählen

7. Resultatives Zählen: Hier wird überprüft, ob die Kinder in der Lage sind, die Gesamtzahl von Objekten in strukturierten und unstrukturierten Mengen zu ermitteln, wobei zeigen, berühren und herumschieben der Holzwürfel hier beim Zählen nicht erlaubt ist. Beispielaufgabe: Der Versuchsleiter legt 19 Holzwürfel in einem Haufen auf den Tisch mit etwas Abstand zwischen den Holzwürfeln und fragt: „, Wie viele Holzwürfel liegen hier?" 


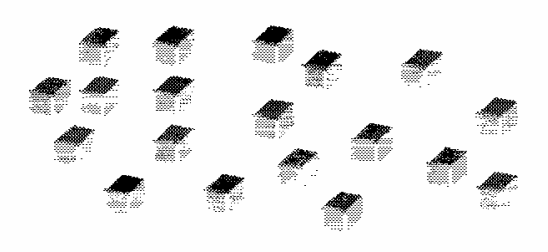

Abb. 7 Beispielaufgabe Resultatives Zählen

8. Allgemeines Zahlenwissen: In alltäglichen Situationen werden Kinder mit Zahlen konfrontiert. Bewältigen sie diese Anforderungen? Diese Komponente bezieht sich auf die Anwendung von Wissen über Zahlen, die in Zeichnungen dargestellt sind. Beispielaufgabe: „Du hast neun Murmeln und verlierst drei davon. Wie viele sind übrig? Zeige mir das Bild mit der richtigen Anzahl von Murmeln. “

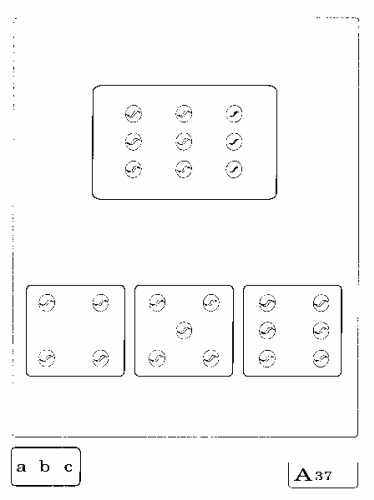

Abb. 8 Beispielaufgabe Allgemeines Zahlenwissen

Zur Erinnerung sei nochmals bemerkt, dass die vier ersten Komponenten aus den Arbeiten von Piaget abgeleitet wurden, aber mit dem doch sehr wichtigen Unterschied, dass den Kindern bei Bedarf das Zählen jederzeit gestattet wird. Dies gilt insbesondere bei den Aufgaben der „Eins-zu-eins-Zuordnung“.

Da alle unsere Untersuchungsdaten über die frühen mathematischen Kompetenzen auf die Aufgaben des OTZ beruhen, sehen wir es als sinnvoll an, die Herkunft und die Güte dieses Tests etwas breiter und detaillierter zu dokumentieren.

Die Entwickler des Tests haben sich bei der Ausarbeitung der Aufgaben inspirieren und anregen lassen von in Holland vorhandenen Materialien wie mathematische Lehrprogramme für jüngere Kinder sowie Schul- und Lehrerhandbüchern. Ebenfalls wurde die einschlägige Literatur zu diesem Thema konsultiert (Fuson 1988; Geary 1994; Skemp 1989). So wurden zu jeder Komponente 15 Fragen entwickelt, die von drei unabhängi- 
gen, im Bereich frühe Mathematik ausgewiesenen Experten auf ihren Inhalt geprüft und die dann gemäß ihrem vermeintlichen Schwierigkeitsgrad geordnet wurden. Zum Beispiel wurde eine Aufgabe, in der es um die Zahlen Eins bis Fünf ging, vor einer Aufgabe angeordnet, in der die Zahlen Sechs und größer vorkamen. Eine weitere sehr wichtige und unabdingbare Erprobungsmöglichkeit ist das Durchspielen des Tests vor Ort. So wurden die Aufgaben in Pilotstudien eingesetzt und in qualitativer und quantitativer Hinsicht getestet. Es wurde sehr viel Wert darauf gelegt, dass die Aufgaben für alle Kinder verständlich und die verwendeten Bilder kindgerecht und attraktiv sind. In qualitativer Hinsicht wurde dahingehend gearbeitet, herauszufinden, welche Aufgaben für einen Test unbrauchbar waren, weil sie zu schwer oder zu leicht waren. Im Weiteren konnte zum Beispiel herausgefunden werden, welche Testaufgaben wirklich die mathematische und nicht die sprachliche Kompetenz messen. Zweimal wurde dieses Verfahren durchgeführt: Am Schluss hatten die Autoren einen Aufgabenpool mit 120 Aufgaben, mit deren Hilfe das Konstrukt „früher Zahlbegriff“ gemessen werden kann.

Die Erfahrung zeigt nun aber, dass unmöglich 120 Aufgaben jeder Versuchsperson vorgelegt werden konnte. Pilotversuche haben gezeigt, dass 40 Aufgaben das allerhöchste Maß darstellte, um mit Kindern von 4 bis 7 Jahren ein vernünftiges Interview durchzuführen. Diese Tatsache zwang die Autoren zur Verwendung eines unvollständigen Tests. Die 120 Aufgaben wurden darum aufgeteilt in sechs Teiltests mit insgesamt 40 Aufgaben (je 5 Aufgaben pro Komponente), dergestalt, dass jede Aufgabe in zwei Teiltests vorkam. Auf diese Weise war jeder der sechs Teiltests mit den andern durch diese gemeinsamen Aufgaben verbunden. Durch dieses Design wurde es „möglich, die Aufgabenparameter beim Prozess der Teileichung durch die gemeinsamen Aufgaben auf einem einzigen Kontinuum zu lokalisieren“ (van Luit, van de Rijt, Hasemann 2001, 10). 823 holländische Kinder (395 Buben und 428 Mädchen) aus 25 Grundschulen waren die Versuchspersonen. Um die Kinder in den gleichen Altersstufen miteinander vergleichen zu können, wurden die Kinder in sechs Altersgruppen I bis VI eingeteilt. Diese sechs Altersgruppen sind in der nachfolgenden Tabelle 1 aufgelistet. Zur Erinnerung: 5;07 5;12 bedeutet, dass die Kinder in dieser Altersstufe III sich im Altersintervall von 5 Jahren und 7 Monaten bis 5 Jahren und 12 Monaten befinden.

\begin{tabular}{|l|c|}
\hline Gruppe I & $4 ; 07-4 ; 12$ \\
\hline Gruppe II & $5 ; 01-5 ; 06$ \\
\hline
\end{tabular}




\begin{tabular}{|l|c|}
\hline Gruppe III & $5 ; 07-5 ; 12$ \\
\hline Gruppe IV & $6 ; 01-6 ; 06$ \\
\hline Gruppe V & $6 ; 07-6 ; 12$ \\
\hline Gruppe VI & $7 ; 01-7 ; 06$ \\
\hline
\end{tabular}

Tab. 1 Die Kinder werden in sechs Altersgruppen eingeteilt.

Die Ergebnisse des ersten Testlaufes zeigten zum Beispiel, dass eine Aufgabe von allen 823 Versuchspersonen richtig gelöst wurde; diese Aufgabe also keine Informationen über das Niveau der Zahlbegriffsentwicklung der Kinder gab und darum direkt aus dem Aufgabenpool fiel. Die weitere, sehr aufwendige und komplizierte Analyse der verbliebenen Ergebnisse (erwähnt sei die Item Responce Theory ${ }^{17}$ ) führte zum Ausschluss von weiteren 39 Aufgaben.

In einem zweiten Schritt wurden die Schwierigkeiten der verbliebenen Aufgaben ermittelt unter anderem mit dem Ziel, dass innerhalb der Komponenten die Aufgabenschwierigkeit ansteigen sollte. (Erwähnt sei hier der $\mathrm{R}_{1 \mathrm{c}}$-Test.)

Das Resultat dieser sehr aufwendigen Tests ist ein Pool von 80 Aufgaben, aus dem Aufgabengruppen entnommen werden können, mit deren Hilfe man die frühe Zahlbegriffsentwicklung von Kindern einschätzen kann. Aus diesem Aufgabenrepertoire wurden dann zwei Testformen A und B definiert, die zusammen den gesamten Osnabrücker Test zur Zahlbegriffsentwicklung (OTZ) ausmachen. Der OTZ erfüllt im Übrigen die bei Lienert und Raatz (1998, 7ff) geforderten Testkriterien Objektivität, Reliabilität und Validität $^{18}$ (Vergleiche auch Testmanual des OTZ 2001, 30ff).

Die zwei Testformen A und B wurden geschaffen um zum einen - wie wir gesehen haben - die Kinder nicht zu überfordern, und zum zweiten, um die Möglichkeit zu haben,

${ }^{17}$ Das Item Response Modell spezifiziert die Wahrscheinlichkeit einer Beziehung zwischen einer latenten Eigenschaft [hier: der frühe Zahlbegriff] und den Antworten auf die Testfragen bei einer bestimmten Population (van Luit \& van de Rijt \& Hasemann 2001, 10).

${ }^{18}$ Unter der Objektivität eines Tests wird der Grad verstanden, in dem die Ergebnisse eines Tests unabhängig vom Untersucher sind. Ein Test wäre also vollkommen objektiv, wenn verschiedene Untersucher bei denselben Probanden zu gleichen Ergebnissen gelangten.

Unter der Reliabilität oder Zuverlässigkeit eines Tests versteht man den Grad der Genauigkeit, mit dem er ein bestimmtes Verhaltensmerkmal misst, gleichgültig, ob er dieses Merkmal auch zu messen beansprucht (Problem der Validität). Ein Test wäre also vollkommen reliabel, wenn die mit seiner Hilfe erzielten Ergebnisse den Probanden fehlerfrei beschreiben.

Unter der Validität oder Gültigkeit eines Tests versteht man den Grad der Genauigkeit, mit dem dieser Test diejenige Verhaltensweise, die er messen soll, auch tatsächlich misst. Ein Test ist demnach vollkommen valide, wenn seine Ergebnisse einen unmittelbaren und fehlerfreien Rückschluss auf den Ausprägungsgrad des zu erfassenden Verhaltensmerkmals zulässt, wenn also der individuelle Testpunktwert eines Probanden diesen auf einer Merkmalsskala eindeutig lokalisiert. 
dieselben Kinder zu einem späteren Zeitpunkt nochmals mit neuen Aufgaben testen zu können. Es ist möglich, dass die Testergebnisse eines Kindes durch besondere Testumstände beeinflusst wurden und möglicherweise unglaubwürdige Resultate ermittelt wurden, zum Bespiel eine extrem hohe oder tiefe Punktezahl. Bei solch einem unerwarteten Testergebnis kann diesem Kind einige Tage später die jeweils andere Testform vorgelegt werden. Falls die ersten Testumstände keinen Einfluss auf das Testresultat ausgeübt haben sollten, ist im zweiten Durchgang mit vergleichbaren Ergebnissen zu rechnen; andernfalls sollte sich in diesem zweiten Durchgang im Wesentlichen das erwartete Ergebnis einstellen.

Eine wichtige Einsatzmöglichkeit des OTZ ist nebst der Ermittlung des individuellen Entwicklungsniveaus, das sich durch den Vergleich der Leistung dieses Kindes mit der einer Normgruppe von Kindern gleichen Alters bestimmen lässt (für unsere Fragestellung der individuellen Problemlösestrategie ein zentrales Untersuchungsergebnis) auch die Möglichkeit der Evaluation der Wirksamkeit von mathematischen Förderprogrammen. Wird der Test vor und nach einem solchen Förderprogramm eingesetzt, so lässt sich ermitteln, wie sich die mathematische Kompetenz des Kindes verändert hat und wie sich der Zahlbegriff entwickelt hat (vgl. van Luit \& van de Rijt \& Hasemann 2001, 7).

Nebst der sehr aufwendigen Testanalyse wurde auf der Basis der Ergebnisse der 823 holländischen Versuchspersonen eine eindimensionale Skala entwickelt, die sowohl die Schwierigkeitsstufen der Aufgaben als auch die Leistungsstufen der Testpersonen darstellt. Die folgende Abb. 9 zeigt die eindimensionale Skala basierend auf den Testergebnissen der 823 holländischen Kindern (entnommen aus van Luit, van de Rijt, Hasemann 2001, 7ff). 


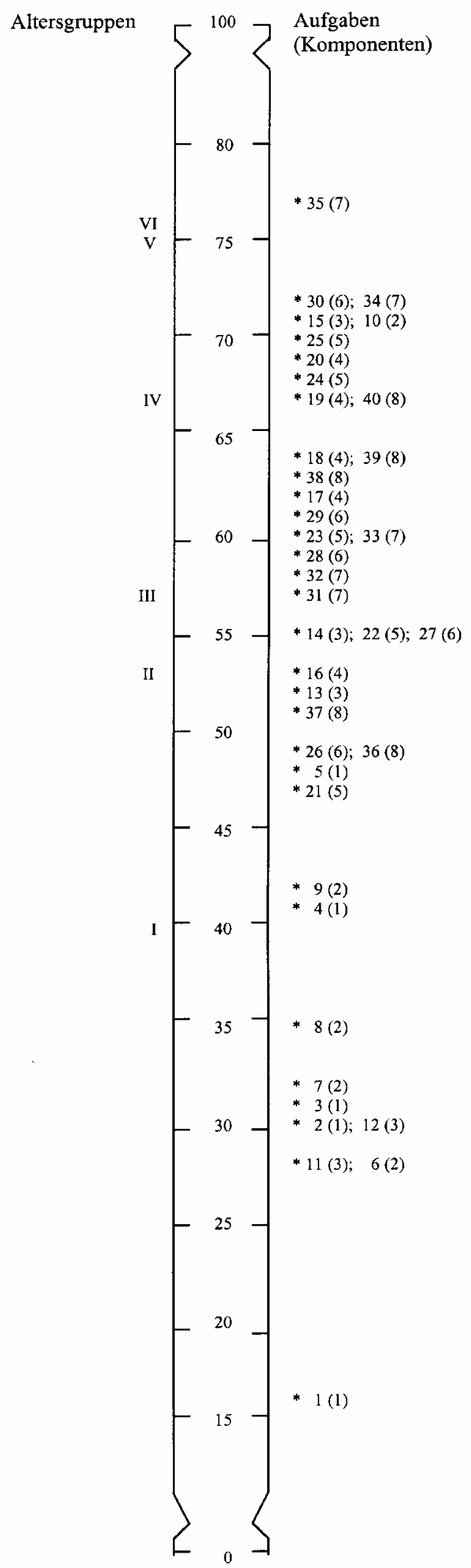

(1) Aufg. 1 - 5: Vergleichen

(2) Aufg. 6-10: Klassifizieren

(3) Aufg. 11-15: Eins-zu-einsZuordnen

(4) Aufg. 16 - 20: Nach Reihenfolgen ordnen

(5) Aufg. 21 - 25: Zahlwörter gebrauchen

(6) Aufg. 26 - 30: Strukturiertes Zählen (mit Zeigen)

(7) Aufg. 31 - 35: Resultatives Zählen (ohne Zeigen)

(8) Aufg. 36 - 40: Allgemeines Zahlenwissen

Abb. 9 Kompetenzskala 
Im Test wird für eine richtig gelöste Aufgabe ein Punkt vergeben. Um die Lesbarkeit zu erleichtern, wird dann aber das Gesamtergebnis linear transformiert (mit dem Faktor 2.5), sodass die Maximalpunktzahl 100 erreicht werden kann. Die Zahlen I bis VI links der Skala geben die durchschnittlichen Ergebnisse der entsprechenden Altersgruppen im holländischen Test wieder. Die mit einem Sternchen versehenen Zahlen rechts der Skala geben die Lösungswahrscheinlichkeit der betreffenden Aufgabe an. Das heißt, ein Kind, das ein bestimmtes Niveau (links abzulesen) erreicht, wird eine Aufgaben mit 50\% Wahrscheinlichkeit lösen, die unmittelbar rechts aufgeführt ist. Die Aufgaben, die darunter liegen, wird es mit höherer Wahrscheinlichkeit, diejenigen die darüber liegen, mit tieferer Wahrscheinlichkeit lösen. Je weiter vom Kompetenzniveau entfernt, desto mehr unterscheiden sich die Wahrscheinlichkeiten von der 50\%-Marke. Zum Beispiel wird ein Kind der Altersstufe III mit einem Gesamtergebnis von 55 Punkten die Aufgaben $* 14, * 22$ und *27 mit einer Wahrscheinlichkeit von 50\% lösen. Je weiter nun die Nummern nach unten verschoben sind, desto größer ist die Lösungswahrscheinlichkeiten dieser Aufgabe. So wird es die Aufgaben 16, 13, 37 usw. mit immer höherer Wahrscheinlichkeit lösen. Umgekehrt wird das Kind die Aufgaben *31, *32 usw. mit einer Wahrscheinlichkeit von weniger als 50\% lösen können. Je weiter die Nummern nach oben verschoben sind, desto kleiner ist die Lösungswahrscheinlichkeit (vgl. Van de Rijt, Van Luit, Hasemann 2000, 14 - 15).

Vor allem um die Frage zu klären, ob denn individuelle Unterschiede in den Leistungen, die von dieser Skala gemessen werden, altersabhängig sind, d.h. ob denn die Skala Entwicklungsstufen aufzeigt, wurde der Mittelwert $M$ und die Standardabweichung $S D$ der sechs Altersgruppen, getrennt nach Mädchen und Jungen ermittelt ( $N$ Gruppengröße, $R$ Bandbreite $)^{19}$.

\footnotetext{
${ }^{19}$ Die Standardabweichung gibt u.a. das Maß der Streuung der Ergebnisse wieder: etwa zwei Drittel aller $N$ Werte liegen im Intervall von $M-S D$ bis $M+S D$.

Die Bandbreite $R$ gibt das Intervall von niedrigsten zum höchsten Wert wieder
} 


\begin{tabular}{|c|c|c|c|c|c|c|c|c|c|c|}
\hline $\begin{array}{l}\text { Alters- } \\
\text { gruppe }\end{array}$ & \multicolumn{4}{|c|}{ Buben } & \multicolumn{4}{c|}{ Mädchen } & \multicolumn{4}{c|}{ gesamte Gruppe } \\
\hline & $M$ & $S D$ & $N$ & $M$ & $S D$ & $N$ & $M$ & $S D$ & $N$ & $R$ \\
\hline I & 39.39 & 14.36 & 70 & 40.74 & 10.77 & 78 & 40.12 & 12.53 & 148 & $6-72$ \\
\hline II & 52.18 & 13.49 & 65 & 54.56 & 13.73 & 75 & 53.27 & 13.61 & 140 & $17-88$ \\
\hline III & 58.05 & 13.61 & 66 & 56.74 & 10.88 & 64 & 57.34 & 12.16 & 130 & $25-93$ \\
\hline IV & 67.84 & 14.70 & 60 & 66.24 & 13.71 & 74 & 67.04 & 14.19 & 134 & $23-99$ \\
\hline V & 74.89 & 10.58 & 63 & 76.05 & 11.54 & 69 & 75.57 & 11.11 & 132 & $45-97$ \\
\hline VI & 77.33 & 12.48 & 71 & 74.08 & 10.06 & 68 & 75.76 & 11.43 & 139 & $37-100$ \\
\hline
\end{tabular}

Tab. 2 Die Ergebnisse der holländischen Untersuchung nach Altersgruppen getrennt

Durch eine doppelte Varianzanalyse konnten die Autoren zeigen, dass das Geschlecht keinen signifikanten Einfluss auf die Leistung hatte $(\mathrm{F}<1)$, hingegen erwartungsgemäß das Alter $\left(\mathrm{F}_{5.822}=169.27\right.$, $\left.\mathrm{p}<0.01\right)$. Die beim Test erzielten Leistungen ergaben somit einen klaren Trend, der entwicklungsabhängig ist. Die Mittelwerte der sechs Altersgruppen (I bis VI) finden sich übrigens auch in der Abb. 9. Die Skala in dieser Abb. 9 lässt vielerlei Schlüsse zu. Hat zum Beispiel eine Testperson das für eine bestimmte Aufgabe erforderliche Niveau erreicht, kann -wie gezeigt wurde - eine korrekte Antwort und umgekehrt, falls er dieses Niveau nicht erreicht hat, eine nicht korrekte Antwort vorhergesagt werden. Auch können beim Betrachten der durchschnittlichen Leistungen einige Folgerungen hinsichtlich der frühen Zahlbegriffsentwicklung gezogen werden. Die jüngsten Kinder (Altersgruppe I, 4;7 - 4;12) können zwar einige Aufgaben der Komponenten Vergleichen, Klassifizieren und Eins-zu-eins-Zuordnung richtig beantworten, wohingegen das durchschnittliche vierjährige Kinde kaum in der Lage ist, Aufgaben, die sich auf andere Komponenten beziehen, richtig zu beantworten. Die Bandbreite dieser Altersgruppe liegt aber bei 66 (siehe obige Tab. 2). Aus der Skala lässt sich weiter ablesen, dass mehr als 50\% der Kinder der Alterstufe II (5;01 - 5;06) fast alle Aufgaben (bis auf die Aufgaben 10 und 15) der ersten drei Komponenten Vergleichen, Klassifizieren und Eins-zu-eins-Zuordnung richtig lösen können. Hingegen werden nur einige der leichten Aufgaben der Komponenten ,Nach Reihenfolgen ordnen'. ,Zahlwörter gebrauchen', ,Strukturiertes Zählen' sowie ,Allgemeines Zahlenwissen' vom Durchschnitt der Altergruppe II richtig beantwortet. Die große Bandbreite von 71 weist aber auch in dieser Gruppe sehr deutlich auf die enormen interindividuellen Unterschiede hin. Die durchschnittliche Leistungssteigerung von Alterstufe II zur Alterstufe III (5;07 - 5;12) ist eher gering (von 53.27 auf 57.34). Lediglich 4 Aufgaben 
(Nummer 14, 22, 27 und 31) mehr kann das durchschnittliche Kind dieser Altersstufe lösen. Auch hier ist der Unterschied innerhalb der Altersgruppe mit 68 Punkten wieder sehr hoch. In der Alterstufe IV (6;01 - 6;06) ist das Durchschnittskind in der Lage, die meisten Aufgaben zum ,Ordnen nach Reihenfolgen', sowie einige Aufgaben zum ,Resultativen Zählen' und zum ,Allgemeinen Zahlwissen' richtig zu beantworten. Diese Altergruppe weist die größte Bandbreite von 76 Punkten auf. Viele holländische Kinder der Alterstufen V und VI (6;07 - 6;12 resp. 7;01 - 7;06) haben den regulären Anfangsunterricht in Mathematik schon besucht und beantworten fast alle Aufgaben aller acht Komponenten korrekt. Für diese Kinder ist der Test eher zu leicht. Die Bandbreiten von 52 resp. 62 Punkten in diesen zwei Altersgruppen weisen aber darauf hin, dass es bei Schulanfängerinnen und Schulanfängern sehr viele Kinder mit einer weit unterdurchschnittlichen Ausprägung der Zahlbegriffsentwicklung gibt. Mit der Skala in der Abb. 9 kann also, wie oben gezeigt wurde, für jedes Kind die Zahlbegriffsentwicklung relativ zu der ihrer Altersgenossinnen und Altersgenossen ermittelt werden. Mit dieser Information kann dann das Kind zielgerichtet und individuell gefördert werden. Dies war eines der wichtigsten Ziele der Entwickler des Osnabrücker Tests zur Zahlbegriffsentwicklung (OTZ).

\subsection{Interviewtechniken}

Es existieren verschiedene Befragungsmethoden, die zur Analyse von Problemlöseprozessen geeignet scheinen. Als erstes sei das ,laute Denken“ erwähnt. Mit dieser Methode werden die Probanden aufgefordert, alle ihre Gedankengänge verbal zu vollziehen. Während sie die gestellte Aufgabe lösen, sollen sie alles sagen was ihnen einfällt, während dessen der Versuchsleiter sich weit möglichst zurückhält. Diese Methode des lauten Denkens erlaubt nach Ginsburg (1983) nicht nur die Erforschung der komplexen Aktivitäten, die das Problemlösen ausmachen, sondern auch die zugrunde liegenden internen symbolischen Mechanismen. Die dabei gewonnen Daten können als relativ unbeeinflusste Berichte über die Problemlösungen der einzelnen Probanden betrachtet werden, da der Versuchsleiter sich beim ganzen Prozess völlig zurückhalten muss (siehe Hasemann 1986, 23).

Als zweite Befragungsmethode, die dem lauten Denken sehr ähnlich ist, sei im Folgenden die der „klinischen Interviews“ beschrieben. Der Hauptunterschied dieser zwei Me- 
thoden liegt in der Interaktion des Versuchsleiters. Während der laut Denkende im Idealfall seine Gedanken ungestört mitteilt, kommt in den klinischen Interviews dem Dialog zwischen Probanden und Interviewer größere Bedeutung zu.

Entwickelt wurden die „klinische Unterrichtsmethode“ vor allem von Piaget. Diese klinischen Interviews haben bei den Arbeiten von Jean Piaget eine bedeutende Rolle gespielt. Seine Forschungsarbeiten auf dem Gebiet der genetischen Erkenntnistheorie konzentrierten sich vor allem auf die Frage „Was ist Erkenntnis“, die er durch die Beschäftigung mit der Frage „Wie entsteht Erkenntnis?“ beantworten wollte. Piaget's Arbeiten in verschiedenen psychiatrischen Instituten und Kliniken ließen ihn Erfahrung sammeln mit den sog. klinischen Methoden ${ }^{20}$, bei denen der Therapeut versucht, seinen Patienten mit behutsamem Nachfragen zur Offenlegung seiner Gedanken anzuregen. Diese klinische Methode stellte für Piaget in der Folge das Instrument dar, Denkprozesse möglichst gut zu verstehen, bei denen Handlungen und verbale Äußerungen zugrunde liegen. Ein Verdienst von Piaget war, dass er ein psychoanalytisches Verfahren zur Erhebung von Krankheitsbildern für die genetische Erkenntnistheorie und für die Kinderpsychologie nutzbar gemacht hat. Da die Frage nach der Entstehung von Erkenntnis vor allem das Analysieren des Denkens von Kleinkindern beinhaltet, haftete der ersten Fassung der klinischen Methode eine Schwäche an: Kleinkinder haben oft ein mangelhaftes Vermögen, ihre Gedankengänge zu verbalisieren. Aus diesem Grunde entwickelte Piaget die klinische Methode weiter, indem er sich nicht nur auf Fragen und Antworten beschränkte, sondern auch Handlungen mit einbezog. Um dem Denken der Kinder auf die Spur zu kommen, muss der Interviewer Hypothesen über die Gedanken der Kinder bilden, um in einer flexiblen Weise Fragen zu stellen, die sich meistens auf die vorhergehenden Antworten resp. Handlungen beziehen (vgl. Selter \& Spiegel 1997, 101). Auf diese Weise können zielgerichtet und zeitsparend die Gedanken der Kinder während des Lösungsverhaltens untersucht werden. Diese von Piaget entwickelte Methode wird von seinem Lehrer Claparède treffend beschrieben: „Die Neuheit der Methode liegt darin, dass der Interviewer die Antwort des Kindes auf eine Frage nicht einfach registriert, sondern es zum Reden bringt ... Das Ziel dabei ist es, die hinter den Antworten liegenden verborgenen Strukturen aufzudecken. Es handelt sich um eine Art geistiges Abhorchen ... man steckt nicht auf, wenn das Kind eine unverständliche oder widersprüchliche Antwort gibt: im Gegenteil, man versucht mit dieser Methode, den fliehenden Gedanken

\footnotetext{
${ }^{20}$ Daher der Name „klinisches Interview“
} 
des Kindes immer näher zu kommen, bis man das Rätsel ihrer Struktur lösen kann“ (Piaget 1976, Vorwort; in Hasemann 1986, 23).

Eine dritte Untersuchungsmethode, das halbstandardisierte Interview, wird insbesondere darum verwendet, um systematische Vergleiche der Untersuchungsergebnisse zu ermöglichen. Die Aufgaben, die Probleme oder die Fragen werden vorher genau festgelegt und dem Kind genau so vorgelegt. Der weitere Verlauf des Interviews wird danach aber offen gelassen, damit der Interviewer nachfragen und spontan und direkt Hypothesen testen und überprüfen kann. Wesentlich dabei ist, dass der Interviewer darauf achtet, immer ganz auf das Kind einzugehen (Hasemann 1986, 24). Durch diese Art von Tests erhofft man sich objektive Daten, die man in der Folge für Quervergleiche nutzen kann. Die halbstandardisierten Interviews sind sehr zielgerichtet und es ist möglich, in Einzelinterviews, die mit Vorteil videoaufgezeichnet und im Anschluss analysiert werden, genaue Aussagen über Strategien und Auffälligkeiten zu erhalten. Es wird angestrebt, dass alle Probanden mit dieser Interviewtechnik die gleichen Bedingungen vorfinden ${ }^{21}$, was eine gewisse Objektivität der Tests gewährleisten soll. Vor allem die Feinanalyse und die Möglichkeit von Quervergleichen lassen tatsächlich weiterführende Einsichten in das Denken der Kinder zu. Der OTZ operiert im Wesentlichen mit der Technik des halbstandardisierten Interviews. Obwohl der Technik der halbstandardisierten Interviews eine gewisse Starrheit anhaftet, hat sich bei der Datenerhebung gezeigt, dass sehr viele interessante und erhellende Daten gewonnen werden konnten. In Übrigen haben auch die Interviewer selber sehr viele Erkenntnisse für ihre eigene Arbeit als (angehende) Lehrerinnen und Lehrer gewonnen. Die Durchführung von Einzelinterviews, sei es nun ein klinisches oder halbstandardisiertes Interview, stellt für alle Studierenden eine außerordentlich gute Möglichkeit dar, Einsichten zu gewinnen, die für das spätere Unterrichten sehr wertvoll sein können.

Als vierte Untersuchungsmethode sei das standardisierte Interview erwähnt. Im Gegensatz zu den klinischen und halbstandardisierten Interviews ist es bei den standardisierten Befragungen nicht vorgesehen, auf die Antworten der Probanden zu reagieren. Im Gegensatz zu den klinischen Interviews, bei denen Fragen und Antworten offen gehalten werden und im Unterschied zu den halbstandardisierten Befragungen, wo bei vorgegebenen Fragen die Antworten offen sind, ist bei der standardisierten Unterrichtsmethode

\footnotetext{
${ }^{21}$ Es ist uns bewusst, dass gleiche Bedingungen im Sinne von identischen Bedingungen bei derartigen Vorhaben nicht möglich sind, vor allem wenn verschiedene Personen in unterschiedlichen Umgebungen gleiche Tests durchführen.
} 
die Reihenfolge und Wortlaut der Fragen sowie die erwünschten Antworten von Anfang an eindeutig definiert. ${ }^{22}$

${ }^{22}$ Für Piaget war die Methode der standardisierten Interviews zu starr, um der Vielschichtigkeit des menschlichen Denkens gerecht zu werden. Er hielt es für sinnlos, unbekannte Verhaltensweisen durch eine vorher genau festgelegte starre Versuchsplanung erfassen zu wollen. 


\section{$7 \quad$ Eigene Untersuchung}

\subsection{Ziele der Untersuchung}

Wie bereits in der Einleitung erwähnt gibt es fünf Gründe, die vorliegende Untersuchung durchzuführen. Es sind dies die folgenden:

1. Kompetenzerhebung und Kompetenzunterschiede: In den letzten Jahren sind etliche Studien über die mathematischen Kompetenzen von Vorschulkindern und Schulanfängern veröffentlicht worden (Spiegel 1992; Selter 1995; Van den HeuvelPanhuizen 1995; Hengartner/Röthlisberger 1994, Hasemann 1998 u.a.). Oft hat sich dabei gezeigt, dass falsche Vorstellungen über die Leistungsfähigkeit der Kinder sehr verbreitet sind - durchaus auch bei Expertinnen und Experten. Vor allem die unterschätzten oder sogar ignorierten Zählkompetenzen sind in diesen Untersuchungen gut dokumentiert und diese Befunde haben langsam ein Umdenken bezüglich des Anfangsunterrichts ausgelöst ${ }^{23}$. Hengartner/Röthlisberger (1995) haben insbesondere die oben erwähnte Diskrepanz zwischen den Ergebnissen der Kinder und den Erwartungen der Expertinnen sowie die enormen Unterschiede in den Mittelwerten der richtigen Lösungen zwischen den einzelnen Einschulungsklassen ${ }^{24}$ herausgestellt. Diese Unterschiede in den Ergebnissen einzelner Klassen sind auch ein Resultat einer groß angelegten Untersuchung (76 Klassen mit insgesamt 2013 Kindern) des Schroedel-Verlags. In dieser Untersuchung wird betont, dass große Leistungsdifferenzen zwischen den Klassen einen deutlichen Hinweis auf große Leistungsunterschiede zwischen den Schülern allgemein darstellen. „Ein Bild, das sich innerhalb der Makrostruktur der Gesamtheit der Klassen zeigt, kann in der Regel auch auf die Mikrostruktur der Schüler einer Klasse übertragen werden“ (SchroedelVerlag 1998, 1). Selten werden Testergebnisse veröffentlicht, in denen Unterschiede innerhalb einer Altergruppe im Fokus der Betrachtungen stehen und die das Bemühen der Kinder um Lösungen ins Zentrum stellen. Die Kompetenzunterschiede innerhalb einer Altersgruppe genau zu kennen ist aber wichtig u.a. für Längsschnittstudien, um z.B. Beziehungen zwischen interindividuellen Unterschieden in

\footnotetext{
${ }^{23}$ Leider läuft dieser Umdenkprozess sehr schleppend. So zeigt sich zum Beispiel im Kanton BaselLandschaft, dass ein großer Teil der Lehrerschaft wider besseres Wissen bei Althergebrachtem haften bleibt. Die Gründe dazu sind sicher vielfältig und müssen noch genau untersucht werden.

${ }^{24}$ Die Kinder wurden unmittelbar nach der Einschulung von bewährten Praxislehrpersonen getestet und hatten noch keinen Mathematikunterricht genossen.
} 
numerischen Kompetenzen, der frühen Zahlbegriffsentwicklung u.ä. und den späteren Leistungsdifferenzen in Mathematik $\mathrm{zu}$ untersuchen. Ist frühkindliche mathematische Förderung Garant dafür, dass Kinder in der Schule einen besseren Zugang zum Fach Mathematik finden und dass Probleme gar nicht auftreten? Zwischenergebnisse einer noch nicht abgeschlossenen Untersuchung der Universität Oldenburg über die Entwicklung von mathematischen Kompetenzen im Grundschulalter zeigen deutlich, dass Leistungsunterschiede bei Beginn des 1 . Schuljahres bei einer hohen Korrelation auch am Ende des 1. Schuljahres feststellbar und somit sehr stabil sind (Grüssing 2002). Die Autorin kann solche Tendenzen auch in den Klassen 2 und 3 nachweisen und ,es zeigt sich eine große Heterogenität bezüglich der Entwicklung mathematischer Kompetenzen, die im Hinblick auf den Mathematikunterricht immer wieder die Notwendigkeit von Differenzierung schon im Anfangsunterricht zeigt“" (Grüssing 2002, 202). In dieser Arbeit wird darum nebst der allgemeinen Kompetenzerhebung vor allem der Focus auf die Kompetenzunterschiede innerhalb einer Altersgruppe gerichtet.

2. Voraussetzungen: Welche Voraussetzungen müssen erfüllt sein, damit Vorschulkinder numerische Probleme lösen können? Von besonderem Interesse ist dabei die Frage, ob die „Piaget'schen“ Komponenten Vergleichen, Klassifizieren, Eins-zueins-Zuordnung und Reihenfolgen zwingende Voraussetzung für den Erwerb der Zählkompetenz sind und ob umgekehrt schon vorhandene Zählkompetenz Einfluss ausübt bei der Bewältigung der Aufgaben aus dem Repertoire der „Piaget'schen“ Komponenten. Welche Voraussetzungen müssen sonst noch erfüllt sein, um gewisse numerische Aufgaben bewältigen zu können?

3. Vergleich $\mathrm{NL}-\mathrm{D}-\mathrm{CH}$ : Ein weiterer Grund, die Befragung der Kinder durchzuführen, ist die Möglichkeit eines Vergleichs. Wie stehen unserere Schweizer Kindergartenkinder im Vergleich mit Holländischen und Deutschen Kindern? Ist die OTZKompetenzskala (Seite 87, Abb. 9) auch für die Schweizer Kinder anwendbar und ist dieser Test in der Hand der Schweizer Kindergärtnerinnen ein taugliches Mittel, Abklärungen durchzuführen?

4. Unterschiede in der Art des Denkens: Alle Interviews wurden mit Video aufgezeichnet und soweit es bei der (oft wiederholten) Sichtung der Interviews möglich war, wurde versucht, Unterschiede in der Art des Denkens und des Problemlöseverhaltens zu finden. Wie lösen vier- bis siebenjährige Kinder numerische und nichtnumerische Aufgaben und warum sind einige Kinder erfolgreicher als andere, d.h. 
wie unterscheiden sich die Lösungsstrategien relativ starker von deren relativ schwacher Kinder? Es wurden zwar keine expliziten Fallstudien durchgeführt, da dies den Rahmen dieser Arbeit gesprengt hätte, doch lässt sich aus den vorliegenden Interviewtranskripten eine Reihe von Merkmalen herausarbeiten, die dazu beitragen können, unser Wissen über die mathematischen Kompetenzen innerhalb der Altersgruppe zu erweitern. Oft sind nur im Vergleich mit gleichaltrigen Kindern Auffälligkeiten und Strategien für erfolgreiches Lösen von mathematischen Aufgabenstellungen und Problemen zu finden. Wie noch detailliert gezeigt wird, gibt es enorme Unterschiede in der pränumerischen wie auch in der numerischen Leistungsfähigkeit von gleichaltrigen Kindern. Was sind die Gründe für diese großen individuellen Unterschiede?

5. Verbesserung der Lehrerinnenausbildung (als Nebenziel): Ein weiterer Grund diese Untersuchung durchzuführen war, den Studierenden die Möglichkeit zu geben, durch eigene Befragungen Theorien $\mathrm{zu}$ hinterfragen, $\mathrm{zu}$ verifizieren und auf Praxistauglichkeit zu testen. Für die Studierenden war es zu Anfang sehr schwierig, sich bei den Interviews bewusst zurückzuhalten; es ist aber für das spätere Unterrichten wichtig zu lernen, den Moment des Schweigens zu akzeptieren, die Denkpausen den Kindern zu lassen. Das halbstandardisierte Befragungskonzept des OTZ lässt es selten zu, den Studierenden einsichtig werden zu lassen, „dass das mathematische Denken von Kindern nicht tendenziell defizitär, sondern oft prinzipiell anders ist“" (Selter/Spiegel 1997, 9). Mit dieser Testreihe kann man selten erkennen, dass die Gedanken der Kinder „,manchmal so intelligent sind, dass wir als Fragende oft große Schwierigkeiten haben, sie in ihrer Originalität und Kreativität zu erkennen“" (Selter/Spiegel 1997, 11). Es kommt sicher vor, dass die Kinder in ihrer inneren Welt logisch richtig argumentieren, für unsere Welt aber ein falsches Resultat liefern. Für den einen Teil der Studierenden (die Kindergarten/Unterstufenlehrkräfte) war es von Interesse zu erfahren, was die Kindergartenkinder schon können und mit welchen Kompetenzen sie dann als Kindergärtnerinnen rechnen können. Der große Teil der Studierenden (es waren vor allem die zukünftigen Primarlehrkräfte) fand es aber interessant und spannend zu erfahren, welche Zahlenkenntnisse und Rechenkompetenzen die Kindergartenkinder haben, und vor allem, welche Folgerungen man für den Erstrechenunterricht ziehen kann. Schulbücher, die auf nicht mehr aktuellen 
Lehrplänen basieren und die noch in den meisten $\operatorname{Schulen}^{25}$ benutzt werden, gehen davon aus, dass die Schulanfänger kein oder nur ein sehr vages Verständnis von der Zahlenwelt haben und erst alles Schritt für Schritt aufbauen und erlernen müssen. Damit die Anfangssituation für alle gleich sei, wird in vielen unserer Schulen praktisch bei Null begonnen. Die allermeisten Schulanfängerinnen und -anfänger sind vom ersten Schultag an in einer hochmotivierten und lernwilligen Stimmung; diese Situation muss optimistisch stimmen und wir alle wollen diese positive Grundhaltung nutzen und erhalten. Eine Voraussetzung, dass das den Grundschullehrkräften gelingt, ist nicht zuletzt die Kenntnis der mathematischen Kompetenzen der Kinder. Wir nehmen die Kinder nicht ernst, wenn wir ihnen nicht bieten, was sie brauchen und wollen. Man stelle sich vor, ein Kind zählt ohne Schwierigkeiten bis 100 und im Unterricht erhält es dann die Aufgabe, den Zahlenzug von 1 bis 10 in der richtigen Reihenfolge zu legen ... ${ }^{26}$. Mit einer solchen Niveausenkung besteht die Gefahr, dass der Mathematikunterricht schon vorhandene arithmetische Fähigkeiten teilweise sogar verkümmern lässt. Durch die noch vielerorts praktizierte Gleich- und Kleinschrittigkeit im Anfangsunterricht entsteht bei vielen Kindern eine Sinnarmut und die mangelnde Herausforderung fördert die Motivation zu eigenem mathematischen Tun in keiner Weise.

\subsection{Methode der Durchführung}

\subsubsection{Anwendung des OTZ}

Der im Kapitel 6.1 beschriebene OTZ eignet sich nicht nur sehr gut für diagnostische Anliegen, sondern auch um allgemeine Kompetenzerhebungen durchzuführen. Wir benutzten den OTZ, um Erkenntnisse über das Verhalten der Kinder bei pränumerischen und numerischen Aufgabenstellungen zu gewinnen. Jedem Kind wurden alle 40 Aufgaben der Testform A des OTZ vorgelegt und die meisten Kinder haben auch alle 40 Fragen beantwortet. Eine durchschnittliche Interviewsequenz dauerte 25 Minuten. Wie wir festgestellt haben, ist das eine Zeitspanne, über die sich die meisten Kinder konzentrie-

\footnotetext{
${ }^{25}$ Im Kanton Basel-Landschaft ist es erlaubt, alternativ zum „Zahlenbuch“ auch das „Aargauer Lehrmittel“ zu benutzen. Der weitaus größte Teil der Primarlehrkräfte benutzt nach wie vor das traditionelle „Aargauer Lehrmittel““.

${ }^{26}$ In gewissen Lehrmitteln sind für viele Wochen gar nur die Zahlen 1 bis 6 vorgesehen (z.B. „Aargauer Lehrmittel“(1995)).
} 
ren können; für ganz junge Kinder bedeutet diese Zeitspanne aber ein oberes Limit. Einige wenige vierjährige Kinder haben zwar bis zum Schluss durchgehalten, waren aber geistig nicht mehr anwesend, haben zum Schein noch mitgespielt und artig die Fragen beantwortet. Es hat sich darum oft als etwas schwierig herausgestellt, gewisse sehr junge Kinder zu ihren mathematischen Fähigkeiten zu befragen. Auch zeigte es sich, dass viele Fragen von den sehr jungen Kindern gar nicht erst verstanden wurden; auf diese Problematik werden wir noch zurückkommen.

\subsubsection{Umfeld und Auswertung}

Wir haben den OTZ in der Schweiz 1998 und 2002 in zwei Etappen mit insgesamt 70 vier- bis siebenjährigen Kindern durchgeführt. Dabei haben wir Kinder in fünf verschiedenen Kindergärten besucht, wobei wir bei der Auswahl der Kindergärten darauf achteten, ein breites Bevölkerungsspektrum abzudecken. So befanden sich zwei Kindergärten in der Stadt, zwei in Agglomerationsgemeinden und eines befand sich in ländlicher Idylle. Obwohl das persönliche Umfeld der Kinder bei Kompetenzabklärungen von großer Bedeutung ist, haben wir das individuelle soziale Milieu der Kinder aus Gründen des Persönlichkeitsschutzes außer Acht gelassen.

Mit der Technik der halbstandardisierten Interviews ${ }^{27}$ gelang es uns sehr gut, die Performanz der Kinder zu registrieren und da jedes Interviews videoaufgezeichnet und (teilweise) transkribiert wurde, war eine anschließende Feinauswertung möglich. Da wir schon in früheren Standortbestimmungen mit Interviews Erfahrung sammeln konnten, waren wir vertraut mit Auswertungen von videoaufgezeichneten Kinderaktivitäten im Bereich nichtnumerischer und numerischer Mathematik ${ }^{28}$. Die Auswertung der Videos genügen aber nicht den Methoden der interpretativen Unterrichtsforschung und sind daher nicht so formalisiert wie bei Voigt (1991) beschrieben.

\footnotetext{
${ }^{27}$ Halbstandardisiert im Sinne von fest vorgegebenen Fragen aber mit freier Antwortmöglichkeit.

${ }^{28}$ Bei diesen früheren Standortbestimmungen haben wir zwei Parcours aufgebaut (Zoo und Zirkus) und darauf viele numerische Situationen geschaffen. (Zum Beispiel vier Löwen im Käfig und zwei Löwenkinder außerhalb des Käfigs.) Offene Fragestellungen erlaubten es den Kindern, frei ihre Gedanken zu äußern und es entwickelte sich bei der Durchwanderung des Parcours oft ein Gespräch zwischen Kind und Interviewer. Mit dieser hier angewandten Technik des klinischen Interviews war es möglich, dem Denken der Kinder ein wenig auf die Spur zu kommen (Idee: Hengartner/Röthlisberger).
} 


\subsubsection{Vierfeldertafel}

Um eine qualifizierte und formale Ausgestaltung der Beziehungen zwischen den einzelnen Aufgaben und Komponenten machen zu können, wurde in dieser Arbeit die sogenannte Vierfeldertafel verwendet. Mit dieser in Lienert/Raatz (1998) beschriebenen Methode ist es möglich, den Trennschärfenkoeffizienten $\mathrm{zu}$ bestimmen. Ein hoher Trennschärfenkoeffizient besagt, dass eine entsprechende Aufgabe „gute“ von „schlechten“" Probanden deutlich unterscheidet, indem gute Probanden die Aufgabe meist richtig und schlechte Probanden die Aufgabe meist falsch oder überhaupt nicht beantworten. Ein Trennschärfenkoeffizient von 1 bezeichnet eine maximale Trennschärfe, ein Wert von 0 bringt zum Ausdruck, dass die Aufgabe von guten und schlechten Probanden etwa gleich häufig richtig beantwortet wird. Solche Aufgaben sind dann im Allgemeinen für einen Test unbrauchbar (Lienert/Raatz 1998, 78). Dieses benutzte Testinstrumentarium erlaubt nun Vergleiche zwischen den einzelnen Aufgaben ${ }^{29}$ der beschriebenen acht Komponenten des OTZ. Weitere detailliertere Informationen zur Vierfeldertafel sind ab Seite $143 \mathrm{zu}$ finden.

\subsection{Ergebnisse der Untersuchung}

Da eine Gruppengröße von 70 Kindern nicht repräsentativ ist, sind allgemeingültige Aussagen kaum möglich und so haben alle Aussagen zu Auswertungen nur Tendenzcharakter. Vor allem dann, wenn einzelne Untergruppen miteinander verglichen werden, sind naturgemäß die Gruppengrößen noch kleiner und die Zahlenangaben sind mit noch größerer Vorsicht zu interpretieren. Ich habe darum in den folgenden Auswertungen darauf geachtet, nur solche Vergleiche anzustellen, bei denen die Gruppengrößen nicht zu klein werden und so habe ich im Allgemeinen nur zwischen dem 1. und 2. Kindergartenjahr unterschieden.

\subsubsection{Erste Ergebnisanalyse}

Eine erste Sichtung der Ergebnisse der Untersuchung bestätigt die schon vielfach gemachte Bemerkung, dass die Leistungsunterschiede enorm sind. Die feststellbaren

\footnotetext{
${ }^{29}$ Die als Kennzeichen für bestimmte Kompetenz betrachtet werden.
} 
Kompetenzunterschiede zwischen den Kindern sind sicher teilweise auf Unterschiede im Entwicklungstempo zurückzuführen, aber es gibt auch Unterschiede in der Art des Denkens, auf die ich später noch genauer eingehen möchte. Die nachfolgende Tab. 3 dokumentiert die enormen Kompetenzunterschiede zwischen den Kindern im ersten und zweiten Kindergartenjahr. Dabei ist $M$ der Mittelwert, $S D$ die Standardabweichung, $N$ die Gruppengröße und $R$ die Bandbreite.

\begin{tabular}{|c|c|c|c|c|}
\hline \multirow{2}{*}{$\begin{array}{l}\text { Kindergarten- } \\
\text { jahr }\end{array}$} & \multicolumn{4}{|c|}{ Alle Kinder } \\
\cline { 2 - 5 } & $\boldsymbol{M}$ & $\boldsymbol{S D}$ & $\boldsymbol{N}$ & $\boldsymbol{R}$ \\
\hline 1. Jahr & 39 & 16 & 33 & $10-83$ \\
\hline 2. Jahr & 61 & 20 & 37 & $20-95$ \\
\hline
\end{tabular}

Tab. 3 Die Ergebnisse getrennt nach Kindergartenjahr. Maximalpunktzahl ist 100.

Die 39 (von 100) erreichten Durchschnittspunkte im ersten Kindergartenjahr erhöhen sich um 22 Punkte auf 61 (von 100) Durchschnittspunkte im zweiten Kindergartenjahr. Im Kapitel 9 werde ich versuchen herauszufinden, wie es sich mit den Kompetenzunterschieden genau verhält.

Nicht ganz unerwartet ist, dass die Buben und die Mädchen ungefähr die gleichen Ergebnisse lieferten mit einem Durchschnittswert aller Mädchen von etwa 51 Punkten und dem aller Buben mit etwa 50 Punkten.

\begin{tabular}{|c|c||c|c|}
\hline \multicolumn{2}{|c||}{ Mädchen } & \multicolumn{2}{c|}{ Buben } \\
\hline M & SD & M & SD \\
\hline 51.29 & 21.45 & 50.31 & 20.69 \\
\hline
\end{tabular}

Tab. 4 Die Ergebnisse der 35 Mädchen und der 35 Buben liegen sehr nahe beieinander.

Es sind im Mittel kaum Kompetenzunterschiede zwischen den Mädchen und Buben festzustellen und so werde ich im Weiteren nicht mehr zwischen den Geschlechtern unterscheiden. 


\subsubsection{Die Ergebnisse der acht Komponenten}

Die folgende

Tab. 5 fasst die Ergebnisse bezüglich der einzelnen acht Komponenten zusammen. Punktemaximum ist hier 5 Punkte entsprechend den 5 Aufgaben in den einzelnen Komponenten.

\begin{tabular}{|l|l|l|l|}
\hline Komponenten & $\boldsymbol{M}$ & $\boldsymbol{S D}$ & $\boldsymbol{R}$ \\
\hline Vergleichsbegriffe & 4.2 & 1.1 & $1-5$ \\
\hline Klassifizierung & 3.5 & 1.3 & $0-5$ \\
\hline Eins-Zu-eins-Zuordnung & 2.8 & 1.2 & $0-5$ \\
\hline Reihenfolgen & 1.9 & 1.6 & $0-5$ \\
\hline Zahlwörter & 1.9 & 1.7 & $0-5$ \\
\hline Strukturiertes Zählen & 2.0 & 1.5 & $0-5$ \\
\hline Ergebnisbezogenes Zählen & 1.6 & 1.5 & $0-5$ \\
\hline Allgemeines Zahlenwissen & 2.3 & 1.3 & $0-5$ \\
\hline
\end{tabular}

Tab. 5 Die Ergebnisse der acht Komponenten $(N=70)$.

Die acht Komponenten

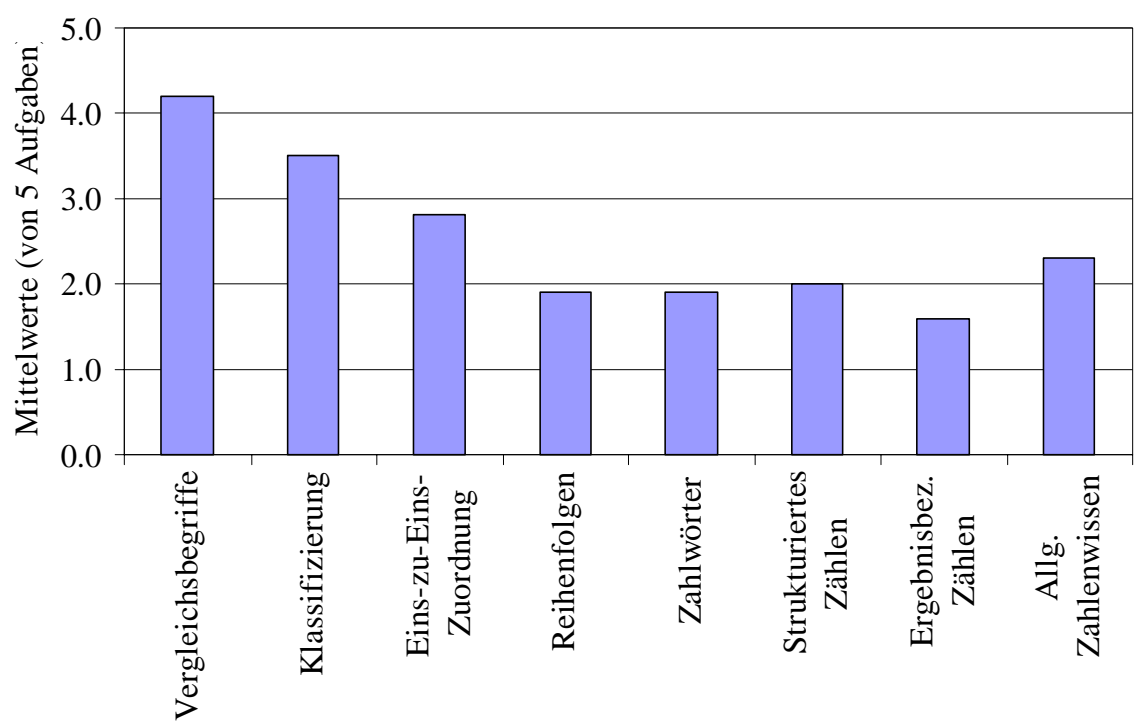

Abb. 10 Die Resultate aller acht Komponenten $(N=70)$.

Es ist ein deutliches Leistungsgefälle innerhalb der einzelnen Komponenten feststellbar.

Es wird notwendig sein, diese krassen Unterschiede genau zu untersuchen. Dies werde 
ich im nächsten Abschnitt tun, indem ich die einzelnen Aufgaben genauer unter die Lupe nehme. Die folgende Tabelle zeigt noch die Ergebnisse der acht Komponenten im Vergleich der Kindergartenjahre ( $N$ 1.KgJ: 33 N 2.KgJ: 37).

\begin{tabular}{|l|l|l|l|l|}
\hline Komponenten & Jahr & $\boldsymbol{M}$ & $\boldsymbol{S D}$ & $\boldsymbol{R}$ \\
\hline Vergleichsbegriffe & 1 & 4.0 & 1.2 & $1-5$ \\
\hline & 2 & 4.3 & 0.9 & $2-5$ \\
\hline Klassifizierung & 1 & 3.2 & 1.5 & $0-5$ \\
\hline & 2 & 3.8 & 1.2 & $0-5$ \\
\hline Eins-Zu-eins-Zuordnung & 1 & 2.3 & 1.1 & $0-5$ \\
\hline & 2 & 3.2 & 1.2 & $1-5$ \\
\hline Reihenfolgen & 1 & 1.3 & 1.2 & $0-3$ \\
\hline & 2 & 2.4 & 1.8 & $1-5$ \\
\hline Zahlwörter & 1 & 0.8 & 0.7 & $0-4$ \\
\hline & 2 & 3.0 & 1.5 & $0-5$ \\
\hline Strukturiertes Zählen & 1 & 1.3 & 1.2 & $0-4$ \\
\hline & 2 & 2.7 & 1.5 & $0-5$ \\
\hline Ergebnisbezogenes Zählen & 1 & 0.9 & 0.9 & $0-3$ \\
\hline & 2 & 2.9 & 1.5 & $0-5$ \\
\hline Allgemeines Zahlenwissen & 1 & 1.7 & 1.2 & $0-4$ \\
\hline & 2 & 2.8 & 1.2 & $0-5$ \\
\hline
\end{tabular}

Tab. 6 Die Ergebnisse getrennt nach dem 1. und dem 2. Kindergartenjahr.

Erste und zweite Jahr im Vergleich

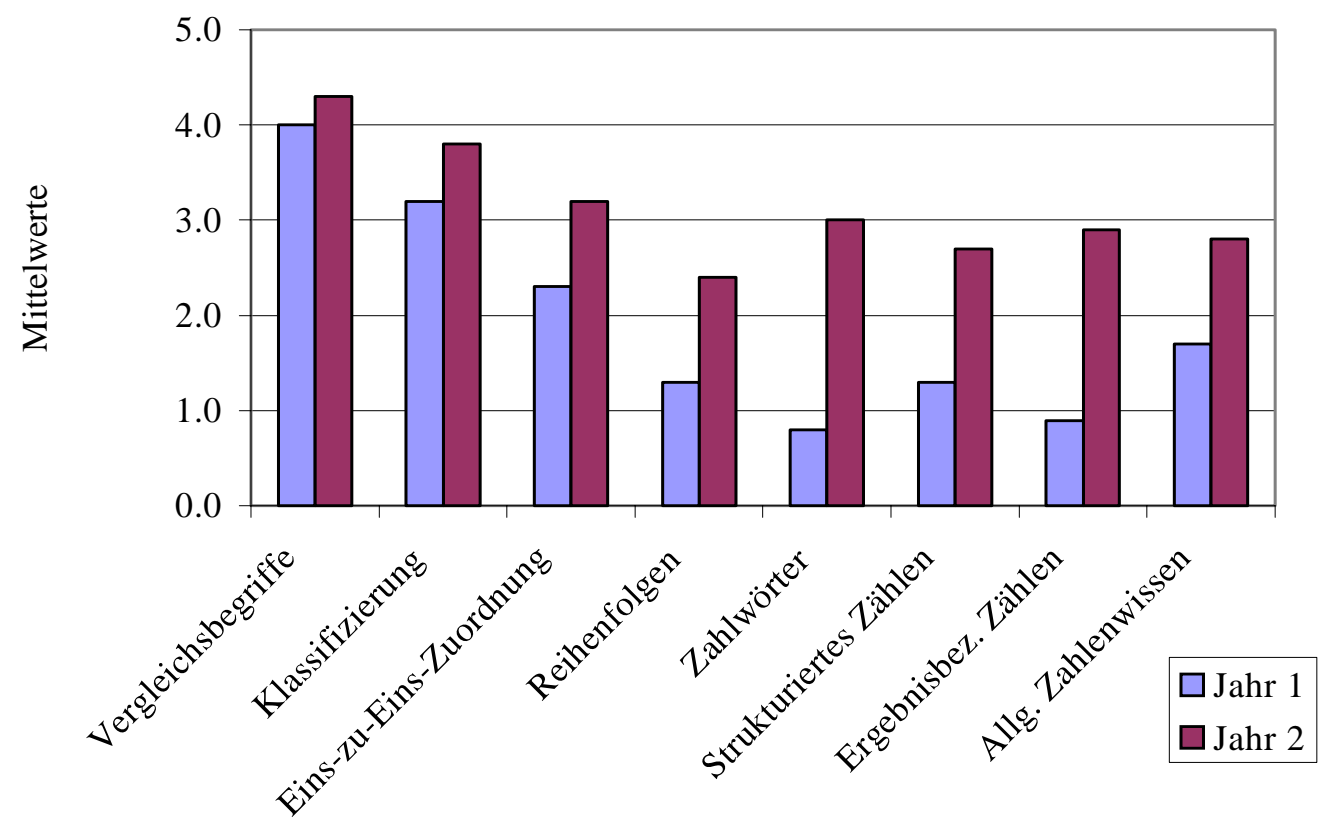


Abb. 11 Der Unterschied der mathematischen Kompetenz zwischen dem 1. und 2. Kindergartenjahr.

Die obige Abb. 11 zeigt die Leistungen der acht Komponenten im Vergleich der zwei Kindergartenjahre. Frappant sind die Unterschiede in den Komponenten Reihenfolgen mit 1.1 Durchschnittspunkten, Zahlwörter mit 2.2, Strukturiertes Zählen mit 1.4, Ergebnisbezogenes Zählen mit 2.0 sowie beim Allgemeinen Zahlenwissen mit 1.1 Durchschnittspunkten. Diese Daten untermauern die These, dass sich die Zählkompetenz zwischen dem 4. und 6. Lebensjahr entwickelt (siehe auch Seite 196, Abb. 61).

\subsubsection{Die Ergebnisse der einzelnen Aufgaben des OTZ}

Die folgende Abbildung zeigt die Ergebnisse aller Kinder bezüglich der einzelnen Aufgaben:

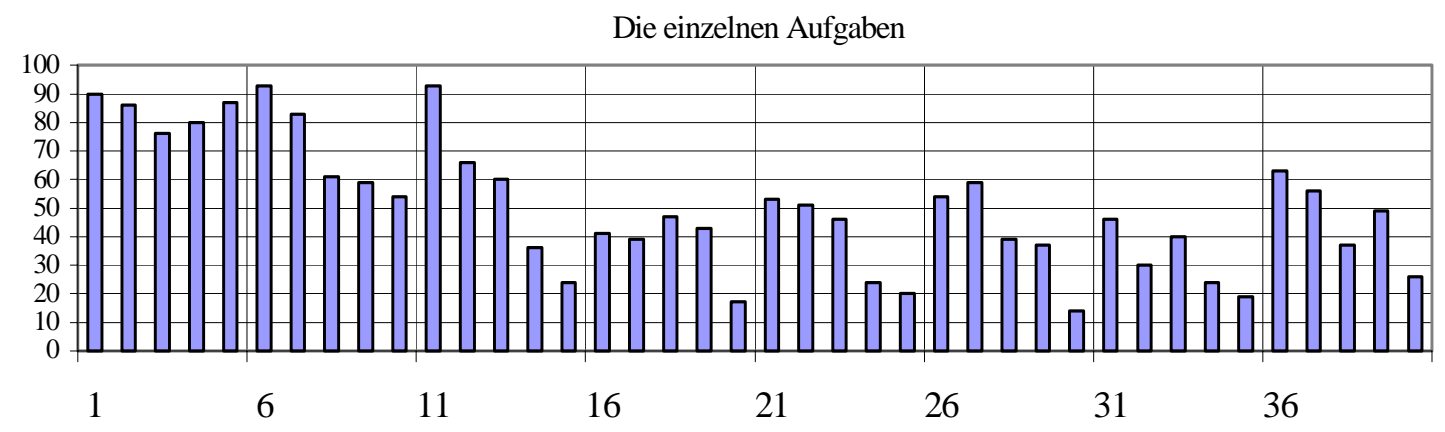

Abb. 12 Die Ergebnisse aller 40 Fragen in Prozent $(N=70)$.

Die Angaben sind Prozentangaben und sie geben Auskunft auf die Frage, wie viel Prozent aller Kinder die betreffende Aufgabe gelöst haben. Die Ergebnisse der fünf Fragen der acht Komponenten sind getrennt durch einen vertikalen Strich. Diese Grafik macht sehr schön deutlich, dass bis auf die Ausnahme „Einordnen in Reihenfolgen“ (Fragen 16 bis 20) immer die ersten Fragen in den Teilaspekten zum Teil sehr deutlich besser beantwortet worden sind als die letzten in der Gruppe. Die Entwickler des Testes hatten aber genau diese Absicht, dass die Kinder mit höherer Wahrscheinlichkeit die ersten Fragen eines jeden Teilaspektes beantworteten. Diese Lösungswahrscheinlichkeiten 
sollten dann innerhalb der Komponente kontinuierlich fallen. ${ }^{30}$ Ganz schön kommt das zum Ausdruck bei der Komponente „Eins-zu-eins-Zuordnung“ mit den Fragen 11 bis 15, wo die Anteile von richtigen Antworten von 93\% ziemlich regelmäßig bis auf $24 \%$ fallen.

Der deutliche Unterschied in der mathematischen Kompetenz zwischen den Kindergärtnerinnen und Kindergärtner im ersten Jahr gegenüber denen im zweiten Jahr wie in der Abb. 11 dargestellt, kommt aber in der Abb. 12 nicht zum Ausdruck. Ich werde aber im Folgenden bei der Besprechung der einzelnen Aufgaben immer auch die Ergebnisse der Erstjährigen mit den Ergebnissen der Zweitjährigen separat betrachten.

Als nächstes werden alle Aufgaben des OTZ einzeln vorgestellt, wobei es mir bei dieser Betrachtung nicht darum geht, die Aufgaben und Fragen des OTZ zu kritisieren, sondern es geht mir bei der Analyse darum herauszufinden, welche Aufgaben von den Kindern für eine weitere genauere Analyse verwendet werden könnten. Außerdem wird das Verhalten der Kinder bei der Bewältigung all dieser Aufgaben beobachtet sowie wie die Resultate zu erklären sind.

\section{Vergleichsbegriffe}

\begin{tabular}{|c|c|c|c|}
\hline AufgabeNr & 1. Jahr & 2. Jahr & Alle \\
\hline \hline 1 & 85 & 95 & 90 \\
\hline 2 & 88 & 84 & 86 \\
\hline 3 & 76 & 76 & 76 \\
\hline 4 & 73 & 87 & 80 \\
\hline 5 & 82 & 92 & 87 \\
\hline
\end{tabular}

Tab. 7 Die Ergebnisse in Prozentangaben getrennt nach dem 1. und dem 2. Kindergartenjahr.

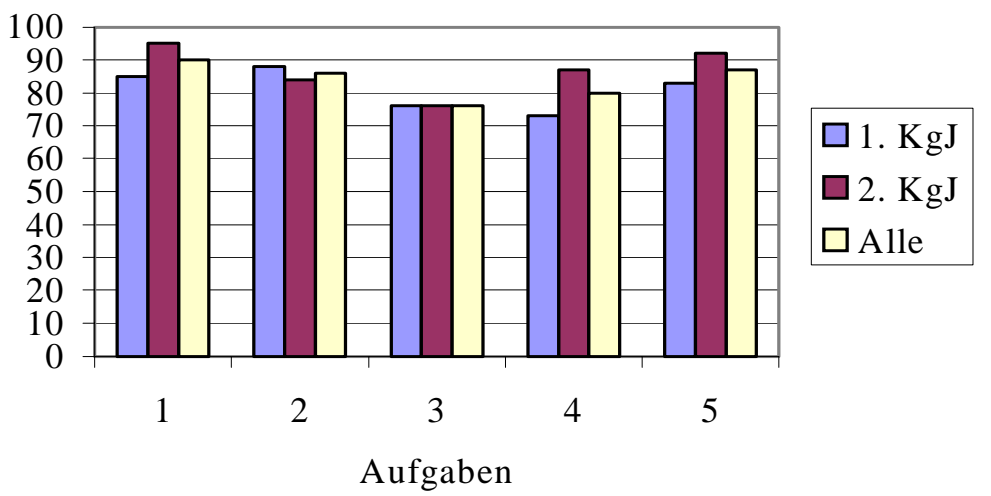

\footnotetext{
${ }^{30}$ Bei den holländischen Testergebnissen fallen die Ergebnisprozente in allen Aspekten mit aufsteigender
} 
Abb. 13 Die Resultate der Komponente Vergleichsbegriffe

Gesamthaft sind alle Aufgaben über Vergleichsbegriffe von über $83 \%$ aller Kinder richtig beantwortet worden, was der höchste Wert aller Komponenten darstellt. Die Unterschiede zwischen dem 1. Kindergartenjahr (1. KgJ) und dem 2. Kindergartenjahr (2. $\mathrm{KgJ})$ bewegen sich in dem zu erwarteten Rahmen.

10 Prozentpunkte ist der Unterschied der Ergebnisse der Kinder im 1. Kindergartenjahr und der Kinder im 2. Kindergartenjahr bei der ersten Aufgabe A1: „Hier siehst du Pilze. Zeige auf den Pilz, der höher ist als diese Blume." [Versuchsleiter/in: (In Zukunft mit Ex. abgekürzt.) Ex. zeigt auf die Blume in dem Kasten oben links auf dem Blatt.] Die Aufgabenstellung ist für die ganz jungen Kinder vielleicht etwas irreführend. Kann hier ein phantasievolles Kind den Rahmen um die Blume zum Beispiel als Fensterrahmen interpretieren und so die Blume aus großer Entfernung sehen? Die älteren Kinder können sehr wohl auch solche oder ähnliche Phantasien haben, scheinen aber eher zu wissen, was die Erwachsenen von ihnen wollen und verhalten sich entsprechend, indem sie ihre Phantasien hinten anstellen und die von ihnen erwartete Antwort geben (Ergebnis: 1. $\mathrm{KgJ} 85 \%$; 2. KgJ 95\%; Gesamt 90\%). Die nachfolgenden Abbildungen sind vom Original herunterkopiert und sind tatsächlich $14 \mathrm{~cm}$ mal $17 \mathrm{~cm}$ groß.

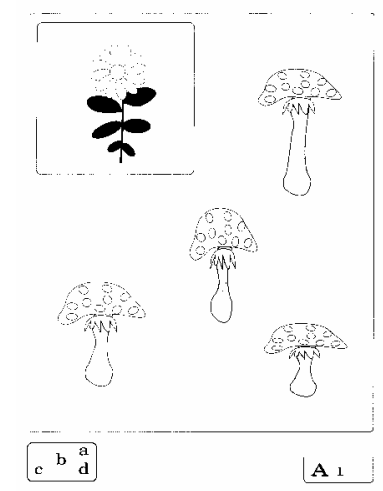

Abb. 14, Aufgabe A1

Aufgabe A2: „Hier siehst du Männer. Zeige auf den Mann, der dicker ist als dieser Mann. " [Ex. zeigt auf den Mann in dem Kasten oben links auf dem Blatt.] Auch diese Aufgabe konnte mit $86 \%$ von den meisten gelöst werden. Interessant ist hier, dass die Kinder im zweiten Kindergartenjahr mit 84\% schlechter abschnitten als die Kinder im ersten Kindergartenjahr. Die Aufgaben A2 und A9 sind die einzigen, bei denen die 
Antworten der Kinder im ersten Kindergartenjahr besser sind als die der Kinder im zweiten Kindergartenjahr (1. KgJ 88\%; 2. KgJ 84\%; Gesamt 86\%).

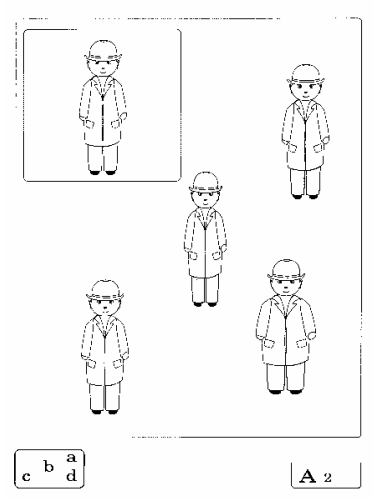

Abb. 15, Aufgabe A2

Die Aufgabe A3: „Hier siehst du Gebäude. Zeige auf das niedrigste Gebäude“ hat oft zu Irritationen geführt. Im Gegensatz zu den Begriffen „höher“ und „dicker“ konnten viele Kinder mit dem Ausdruck „niedrigste“ nichts anfangen. Im Schweizerdeutschen wird das Wort „niedrigste“ sehr selten benutzt und meist ersetzt durch das Wort „kleinste“. So haben wir darum bei Irritationen und Rückfragen bezüglich des Textverständnisses das Wort „niedrigste“ ersetzt durch den Begriff „kleinste“. So haben dann $76 \%$ aller Kinder richtig geantwortet (1. KgJ 76\%; 2. KgJ 76\%; Gesamt 76\%).

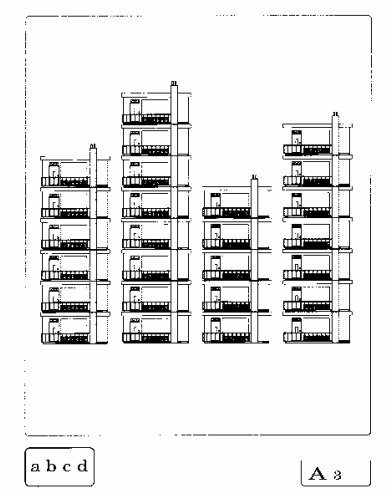

Abb. 16, Aufgabe A3

Das mit 80\% gute Abschneiden bei der Aufgabe A4: „Hier siehst du Indianer. Zeige auf den Indianer, der weniger Federn hat als dieser Indianer mit Pfeil und Bogen" hat vermutlich mit der klar unterscheidbaren Ausdehnung der Federnanordnung zu tun. Die richtige Antwort b hat die deutlich geringste Ausdehnung. (Im Original ist die Breite aller Federn der richtigen Antwort b $6 \mathrm{~mm}$ im Gegensatz zu $12 \mathrm{~mm}$ bei den drei falschen Antworten.) Es wäre interessant zu testen, wie denn die Resultate ausgefallen wären, 
wenn mehr Federn weniger Platz bedurft hätten (1. KgJ 73\%; 2. KgJ 87\%; Gesamt $80 \%)$.

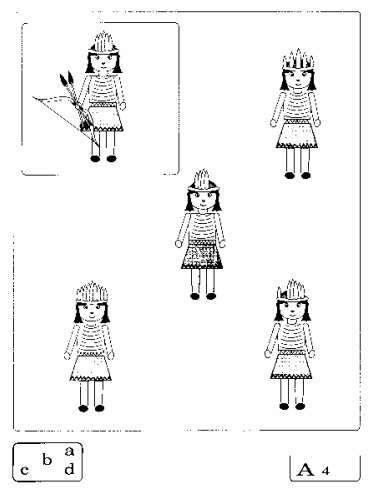

Abb. 17, Aufgabe A4

Im Allgemeinen haben Kinder Schwierigkeiten mit den Begriffen „niedrigste, tiefste, wenigste ..." (siehe Aufgabe A3). Aufgabe A5: „Hier siehst du Kisten mit Murmeln. Zeige auf die Kiste mit den wenigsten Murmeln." Obwohl zu erwarten gewesen wäre, dass die Kinder bei dieser Aufgabe mehr Mühe hätten, haben 87\% der Kinder diese Aufgabe richtig gelöst. Es könnte sein, dass wieder die räumliche Ausdehnung für das gute Resultat verantwortlich ist (1. KgJ 82\%; 2. KgJ 92\%; Gesamt 87\%).

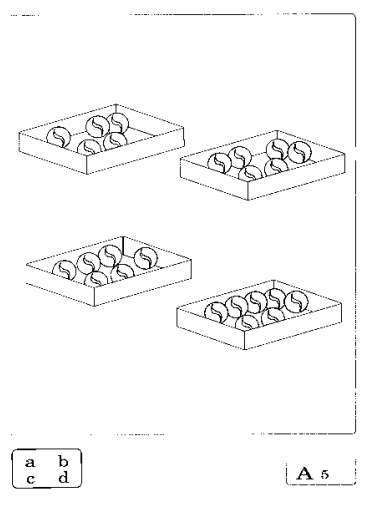

Abb. 18, Aufgabe A5

Kinder sind schon sehr früh im Alltag und im Kindergarten mit Vergleichsituationen konfrontiert, sei es im Freien oder auch beim geführten Spiel beim Bauen, Zeichnen, Bilderbücher anschauen usf. Kinder vergleichen andauernd: „Wer ist schneller als ich?“, „Wer malt die meisten Blumen?“, „Wer ist der größte?“ usw. Die Kinder haben darum in überwiegender Anzahl die Vergleichsaufgaben schnell und richtig gelöst. 


\section{Klassifizierung}

\begin{tabular}{|c|c|c|c|}
\hline AufgabeNr & 1. Jahr & 2. Jahr & Alle \\
\hline \hline 6 & 91 & 95 & 93 \\
\hline 7 & 70 & 95 & 83 \\
\hline 8 & 48 & 73 & 61 \\
\hline 9 & 67 & 51 & 59 \\
\hline 10 & 42 & 65 & 54 \\
\hline
\end{tabular}

Tab. 8 Alle Ergebnisse der Komponente Klassifizierung.

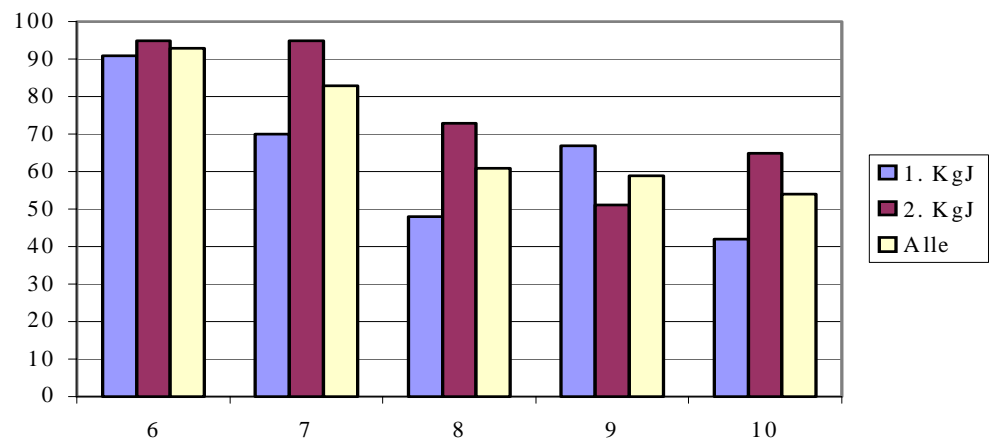

Abb. 19 Die großen Leistungsdifferenzen zwischen den Jahrgängen werden deutlich sichtbar.

Die Aufgabe A6 war wohl die leichteste von allen: „Schau dir diese Bilder an. Was kann nicht fliegen?" 93\% aller Kinder hatten hier Erfolg. Ein derart hoher Wert wurde nur noch bei der Aufgabe A11 erreicht (1. KgJ 91\%; 2. KgJ 95\%; Gesamt 93\%).

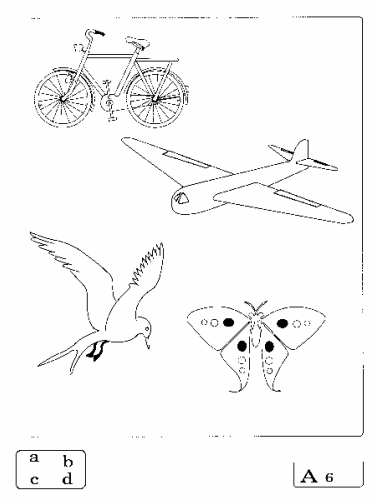

Abb. 20, Aufgabe A6

83\% der Kinder haben die Aufgabe A7 richtig gelöst: „, Schau dir diese Kästen an. Zeige auf den Kasten mit fünf Quadraten, aber ohne Dreieck. "Wenn denn ein Kästchen das richtige ist, dann das ohne Dreiecke und es gibt nur ein Kästchen ohne Dreiecke. Das Zählen kann man sich dann sparen. Und in der Tat: Sehr selten hat hier ein Kind gezählt. 
Auffallend ist das Leistungsgefälle von 25 Prozentpunkten zwischen dem ersten und zweiten Kindergartenjahr. In den Kindergärten wird sehr oft mit geometrischen Formen gearbeitet und gespielt. Davon haben die älteren profitiert (1. KgJ 70\%; 2. KgJ 95\%; Gesamt 83\%).

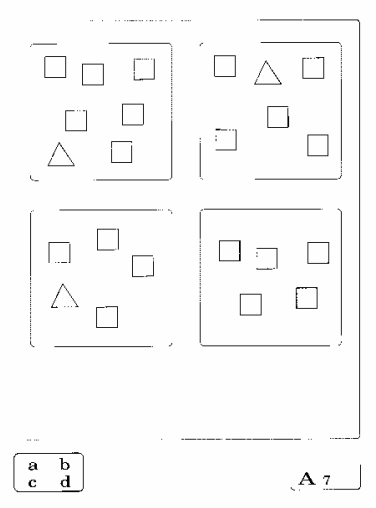

Abb. 21, Aufgabe A7

Der Grund für das relativ dürftige Ergebnis (61\%) der Aufgabe A8: „Schau dir diese Bilder an. Zeige auf alle grauen Kreise 31 “ liegt darin, dass vor allem die Kleinen oft Kreise gezeigt haben, ohne auf das zweite Merkmal „grau“ zu achten oder aber sie haben den kleinsten grauen Kreis vergessen. Wir haben aber nur die Antworten als richtig taxiert, bei denen die Kinder genau die vier grauen Kreise gezeigt haben. Auch damit sind die 25\% Unterschied zu erklären (1. KgJ 48\%; 2. KgJ 73\%; Gesamt 61\%).

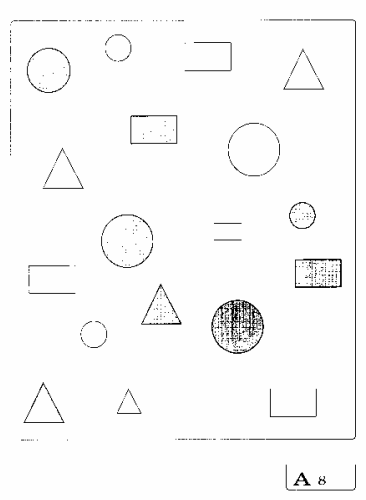

Abb. 22, Aufgabe A8

Die Aufgabe A9: „Hier siehst du Menschen. Zeige auf die Menschen, die eine Tasche, aber keine Brille tragen" ist komplex, weil gleichzeitig zwei Merkmalsausprägungen berücksichtigt werden müssen und verschiedenste Details ablenken. Viele Kinder konnten sich nicht beide Kriterien merken und haben z.B. alle Menschen mit Taschen ge-

${ }^{31}$ Mit „grauen Kreise“ sind hier graue Kreisflächen gemeint 
zeigt. Dass hier die Kleinen beachtliche $67 \%$ erzielten und so weit besser abschnitten als die Älteren, kann ich nicht erklären und ist wohl ein Ausreißer. Es ist dies wie bereits erwähnt mit der Aufgabe A2 die einzige Aufgabe, bei denen die Jüngeren besser abschnitten als die Älteren (1. KgJ 67\%; 2. KgJ 51\%; Gesamt 59\%).

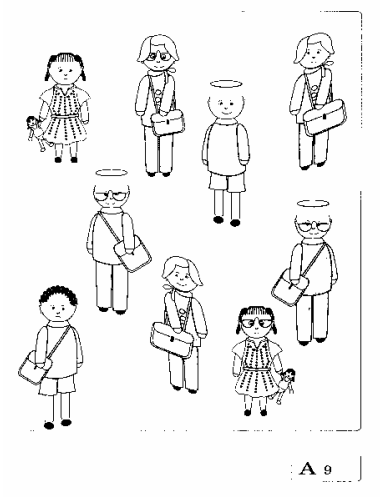

Abb. 23, Aufgabe A9

Die Aufgabe A10 ist mit drei Merkmalsausprägungen sehr komplex, aber auch reizvoll und für die Kinder anregend: „Hier siehst du einen Apfel mit Stiel, ohne Blatt und mit einem Würmchen, das aus dem Apfel herauskommt." [Ex. zeigt auf den Apfel in dem Kasten oben links auf dem Blatt.] „Zeige auf alle Äpfel, die genau gleich aussehen.“ Auch hier stellen wir ein sehr hohes Leistungsgefälle von $23 \%$ zwischen den zwei Altersstufen fest. Für alle zusammen ergibt das eine Erfolgsquote von 54\%, was in Anbetracht der hohen Komplexität der Aufgabe (drei Merkmalsausprägungen) einen hohen Wert darstellt (1. KgJ 42\%; 2. KgJ 65\%; Gesamt 54\%).

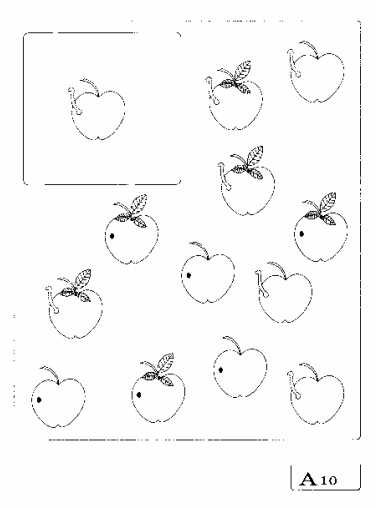

Abb. 24, Aufgabe A10

Auch bei diesen Klassifizierungsaufgaben hatten die Kinder im Allgemeinen keine großen Schwierigkeiten. Im Kindergarten werden oft Situationen geschaffen, bei denen das Sortieren nach bestimmten Eigenschaften eine Rolle spielt, wie zum Beispiel beim Auf- 
räumen: „In diese Kiste kommen alle roten runden Bälle.“ oder beim Verlassen des Zimmers: „Alle Mädchen mit Röcken oder mit rotem Pullover dürfen zuerst gehen.“, usw.

\section{Zuordnungen}

\begin{tabular}{|c|c|c|c|}
\hline AufgabeNr & 1. Jahr & 2. Jahr & Alle \\
\hline \hline 11 & 88 & 97 & 93 \\
\hline 12 & 58 & 73 & 66 \\
\hline 13 & 48 & 70 & 60 \\
\hline 14 & 21 & 49 & 36 \\
\hline 15 & 15 & 32 & 24 \\
\hline
\end{tabular}

Tab. 9 Die Resultate der Aufgaben 11 bis 15.

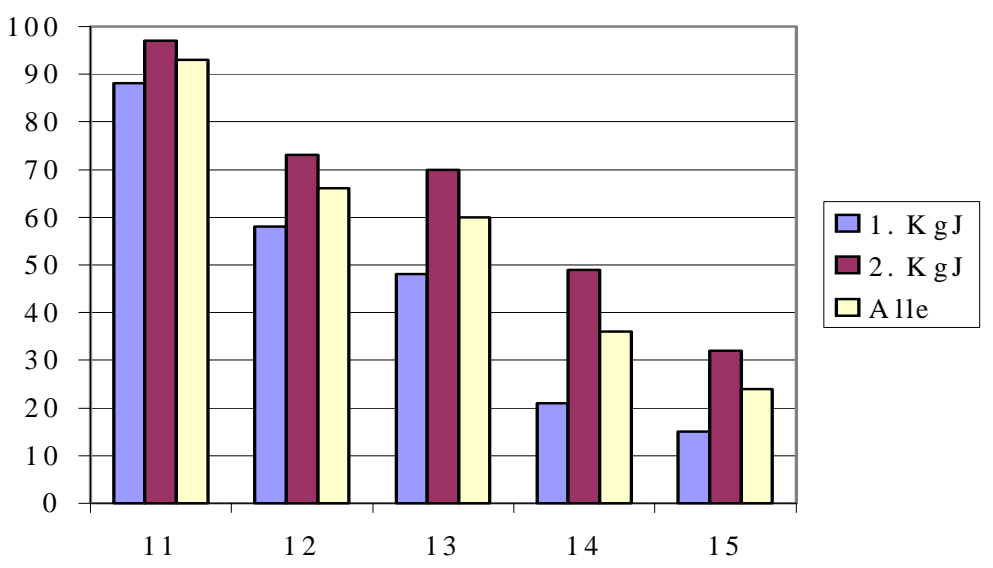

Abb. 25 Die Resultate fallen wie beabsichtigt schön regelmäßig in der Komponente Zuordnungen.

Die Erfolgszahlen fallen in schöner Regelmäßigkeit vom höchsten Wert von allen bei Aufgabe A11 (93\%) auf das tiefe Ergebnis bei Aufgabe A15 (24\%).

Die Aufgabe A11: [Ex. gibt dem Kind 10 Holzwürfel.] „Du hast eine vier gewürfelt“ [Ex. zeigt auf das Zahlbild mit den vier im Kasten] „Kannst du die gleiche Anzahl von Holzwürfeln auf den Tisch legen?" Hier haben alle Kinder eine Chance, auch diejenigen, die überhaupt nicht zählen können. Ich habe Kinder interviewt, die mit der einen Hand auf die Punkte der Vorlage gezeigt haben, und mit der andern Hand die Holzwür- 
fel hingelegt haben. Andere wiederum haben alle Punkte auf der Vorlage mit den Holzwürfeln zugedeckt (1. $\mathrm{KgJ} 88 \% ; 2 . \mathrm{KgJ}$ 97\%; Gesamt 93\%).

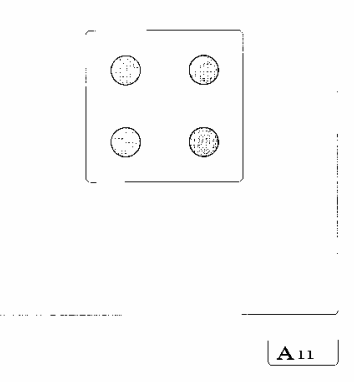

Abb. 26, Aufgabe A11

Diese Eins-zu-eins-Zuordnung wird bei der Frage A12 ein wenig erschwert durch die höhere Anzahl Punkte. Eventuell könnte die Aufgabe durch das Kind selber erschwert werden, indem es eine versteckte Aufforderung zu einer Addition hineininterpretiert.

A12: [Ex. gibt dem Kind 15 Holzwürfel.] „Ich habe mit zwei Würfeln diese Zahlen gewürfelt. Kannst du die gleiche Anzahl von Holzwürfeln auf den Tisch legen?" [Ex. zeigt auf die Zahlbilder mit der fünf und der sechs.] Vor allem Kinder aus dem ersten Kindergartenjahr legten zuerst die Anzahl Würfel des ersten Würfelbildes auf den Tisch, räumten sie dann wieder weg und legten die Anzahl Würfel des zweiten Würfelbildes. In der Aufgabenstellung werden die Kinder aufgefordert, gleich viele Würfel auf den Tisch zu legen, wie abgebildet sind. Wir haben darum nur solche Lösungen als richtig taxiert, bei denen alle 11 Würfel auf dem Tisch lagen. Nur noch 66\% schafften diese Aufgabe. (1. KgJ 58\%; 2. KgJ 73\%; Gesamt 66\%)

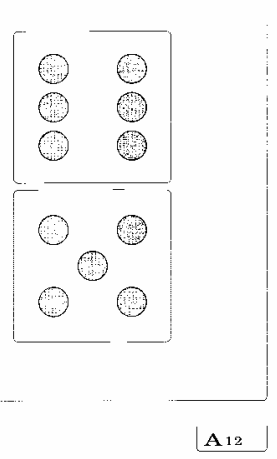

Abb. 27, Aufgabe A12 
Die Aufgabe A13 war für die Interviewer sehr interessant. Hier konnte sehr oft das Ringen der Kinder nach einer Lösung beobachtet werden. A13: [Ex. gibt dem Kind ein Arbeitsblatt und einen Bleistift.] „Hier siehst du Kerzenhalter und Kerzen. In jedem Kerzenhalter sollen Kerzen gesteckt werden. Zeichne Linien von den Kerzen zu den passenden Kerzenhaltern. " Für die Kleinen scheint mir zum mathematischen Problem noch die sprachliche Schwierigkeit hinzuzukommen. Viele Kinder haben den Sinn der Aufgabe nicht verstanden. So hat ein Mädchen begonnen, die Kerzen auszumalen. Diese Zuordnungsaufgabe lösten 60\% aller Kinder (1. KgJ 48\%; 2. KgJ 70\%; Gesamt 60\%).

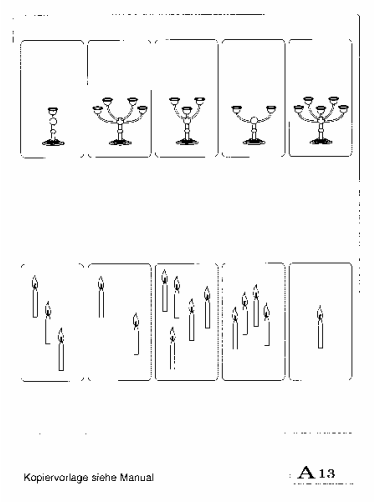

Abb. 28, Aufgabe A13

Die Ergebnisse der Aufgabe A14: [Ex. gibt dem Kind ein Arbeitsblatt und einen Bleistift.] „Hier siehst du drei Bilder mit Hühnern und Eiern. Kannst du das Bild finden, in dem jedes Huhn jeweils ein Ei gelegt hat? Du darfst Linien zeichnen "wären nach meinen Beobachtungen noch schlechter ausgefallen, wäre die richtige Antwort $\mathbf{b}$ nicht in der Mitte platziert worden und das Verhältnis der Länge, die alle Hühner auf einer Linie beanspruchen, zu der Länge der Eier auf einer Linie nicht zu Gunsten der richtigen Antwort ausgefallen. Dieses Verhältnis beträgt bei a 1.25 , bei b 1.13 und bei c 1.68 . Je mehr sich dieses Verhältnis der Zahl Eins nähert, desto mehr nähert sich die Länge der Hühnerreihe derjenigen der Eierreihe. Etliche Kinder haben sich von diesen zwei Tatsachen leiten lassen und zeigten ganz spontan, ohne genaueres Hinsehen, auf das mittlere richtige Bild. Die Kinder aus dem ersten Kindergartenjahr haben es dann mit diesem spontanen Zeigen bewenden lassen, während Kinder aus dem zweiten Kindergartenjahr vorsichtshalber Zuordnungsstriche gezeichnet haben (1. KgJ 21\%; 2. KgJ 49\%; Gesamt $36 \%)$. 


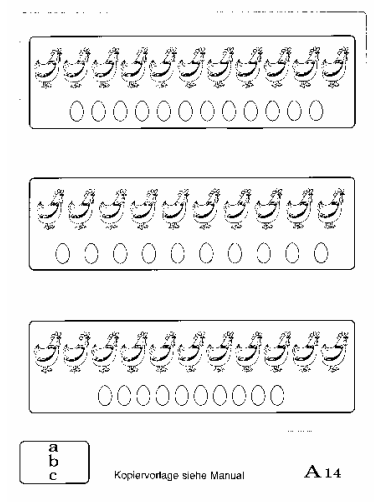

Abb. 29, Aufgabe A14

Bei der Aufgabe A15: „Hier siehst du fünfzehn Luftballons“ [Ex. zeigt auf die Luftballons in dem Kasten oben links auf dem Blatt.] „Zeige auf den Kasten, in dem genau so viele Punkte sind wie hier Luftballons. “ sind vor allem viele Kleine überfordert. Die meisten haben die Information, dass es denn 15 Ballone sind, überhaupt nicht verarbeitet und/oder vielleicht gar nicht verstanden. Andere zählten die Ballone nochmals ab, obwohl die Anzahl Ballone in der Aufgabenstellung explizit erwähnt ist. Wieder andere erfassten zwar die Fünfergruppierung der Lösungsvorschläge, konnten aber nichts damit anfangen (1. KgJ 15\%; 2. KgJ 32\%; Gesamt 24\%).

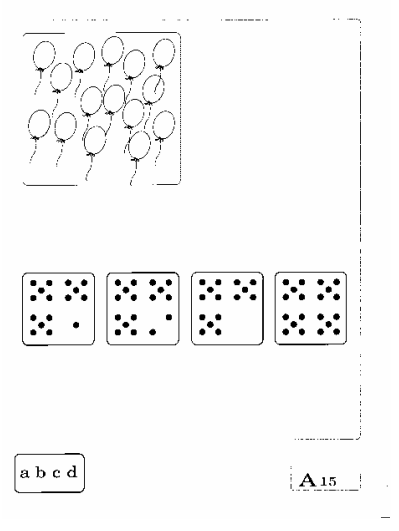

Abb. 30, Aufgabe A15

Ein sicheres Beherrschen einer Ein-zu-eins-Zuordnung ist eine wesentliche Basiskompetenz des Zählens. Einfache Zuordnungsaufgaben sind für alle Kinder gut gelöst worden. Mit steigendem Schwierigkeitsgrad sind die Resultate aber schnell bescheidener geworden, was darauf schließen lässt, dass die Basis für einen sicheren Zählvorgang nicht gelegt ist. 


\section{Einordnen in Reihenfolgen}

\begin{tabular}{|c|c|c|c|}
\hline AufgabeNr & 1. Jahr & 2. Jahr & Alle \\
\hline \hline 16 & 33 & 49 & 41 \\
\hline 17 & 33 & 43 & 39 \\
\hline 18 & 30 & 62 & 47 \\
\hline 19 & 27 & 57 & 43 \\
\hline 20 & 6 & 27 & 17 \\
\hline
\end{tabular}

Tab. 10 Ergebnisse: Einordnen in Reihenfolgen

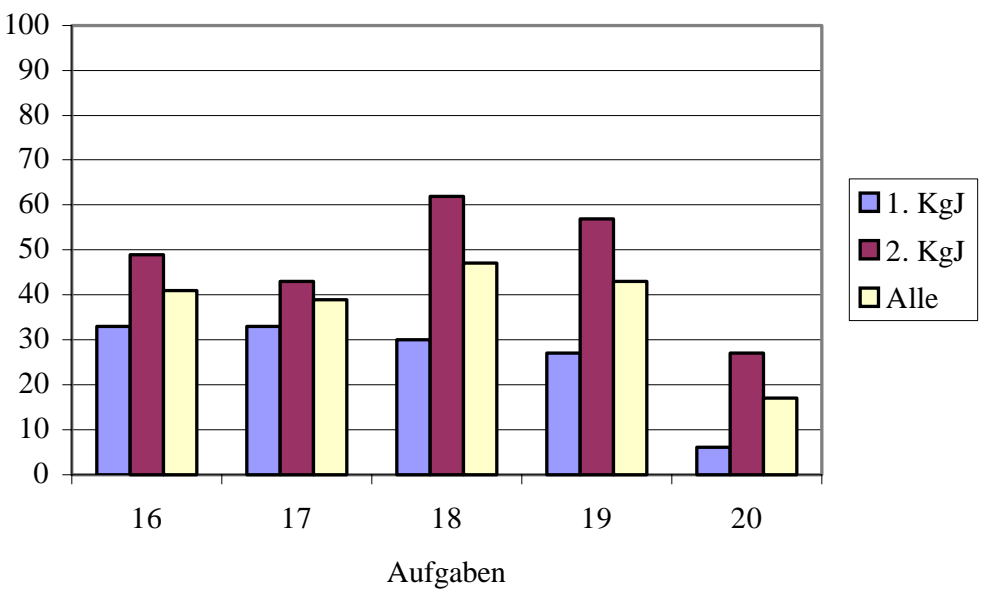

Abb. 31 Hier fallen die Ergebnisse nicht mit aufsteigender Aufgabennummer.

Diese Komponente bildet insofern eine Ausnahme, dass die Anzahl richtiger Antworten nicht mit aufsteigender Aufgabenummer fällt. Das Bestreben war ja, leichtere Aufgaben an den Anfang zu stellen und dann den Schwierigkeitsgrad zu erhöhen. Also ist der Schwierigkeitsgrad der Aufgaben offenbar nicht für die Schweizer Kinder gültig. Zum Beispiel lösten die Aufgabe A17 39\% aller Kinder, hingegen 47\% die Aufgabe A18.

Die Aufgabe A16: „Hier siehst du Kästen mit Äpfel. Zeige auf den Kasten, in dem die Äpfel von groß nach klein geordnet sind. “ wurde von $41 \%$ aller Kinder richtig gelöst. Ich habe festgestellt, dass auch hier etliche Kinder Schwierigkeiten mit der Aufgabenstellung an sich hatten. Haben diese Kinder dann tatsächlich das gegebene Problem gelöst oder hatten sie etwas ganz anderes im Sinn? Einige Kinder aus dem ersten Kindergartenjahr zeigten denn auch nur auf einzelne Äpfel (1. KgJ 33\%; 2. KgJ 49\%; Gesamt $41 \%)$. 


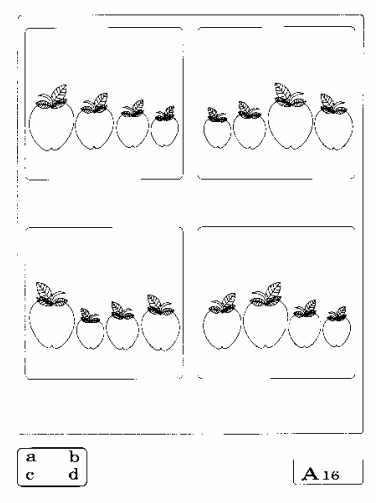

Abb. 32, Aufgabe A16

Aufgabe A17: „Hier siehst du Kästen mit Zuckerstangen. Zeige auf den Kasten, in dem die Zuckerstangen von dünn nach dick geordnet sind." Auch hier gelten wie bei der vorhergehenden Aufgabe die Vorbehalte bezüglich der Sprache und dem Verständnis (1. $\mathrm{KgJ} 33 \%$; 2. KgJ 43\%; Gesamt 39\%).

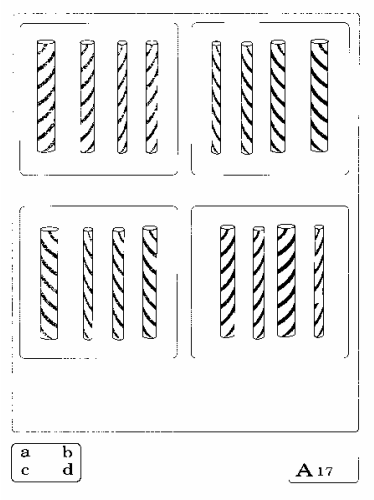

Abb. 33, Aufgabe A17

Aufgabe A18: „Hier siehst du Kästen mit Murmeln. Zeige auf den Kasten, in dem die Murmeln von klein und hell nach groß und dunkel geordnet sind. " Beim näheren Hinsehen erkennt man, dass die zwei Ordnungsmerkmale es den Kindern leichter machen, die richtige Antwort zu nennen. Wenn sie von hell nach dunkel ordnen, haben sie automatisch auch von klein nach groß geordnet und umgekehrt. Also können sie auf zwei Wegen zur richtigen Antwort kommen. Diesen Umstand haben vor allem die älteren Kinder ausgenutzt (62\% richtigen Antworten bei Aufgabe A18 gegenüber nur 49\% richtigen bei Aufgabe A16). Viele aus dem ersten Kindergartenjahr fixierten sich aber trotzdem auf die zwei Ordnungsmerkmale und kamen so wegen dieser Komplexität nicht mit der Aufgabe zurecht und konnten sich für keines der Kästchen entscheiden (1. KgJ 30\%; 2. KgJ 62\%; Gesamt 47\%). 


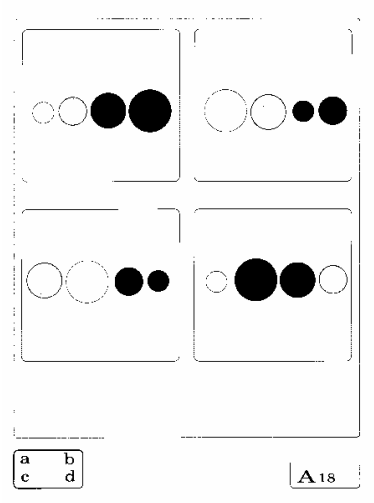

Abb. 34, Aufgabe A18

Aufgabe A19: [Ex. gibt dem Kind ein Arbeitsblatt und einen Bleistift.] „Hier siehst du Hunde. Jeder Hund soll einen Stock bekommen. Ein großer Hund bekommt einen großen Stock und ein kleiner Hund bekommt einen kleinen Stock. Zeichne Linien von den Hunden zu den Stöcken, die sie bekommen. "Hier haben die Kinder keine Verständnisprobleme gehabt. Ein kleiner Hund bekommt einen kleinen Stock; wer verlangt aber, dass der kleinste Hund den kleinsten Stock bekommt; es genügt doch ein kleiner Stock! Viele kleine Kinder aus dem ersten Kindergartenjahr waren mit dem Linienzeichnen wieder überfordert (1. KgJ 27\%; 2. KgJ 57\%; Gesamt 43\%).

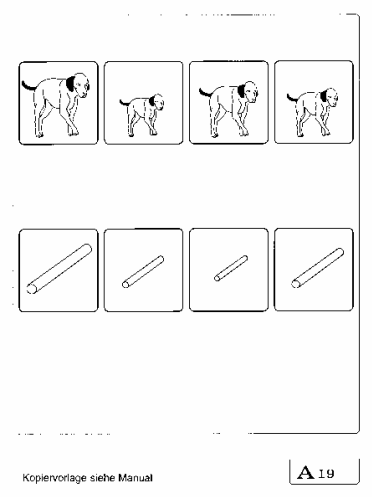

Abb. 35, Aufgabe A19

Für eine Untersuchung in der Schweiz scheint mir die Aufgabe A20 denkbar ungeeignet. A20: „Hier siehst du Stapel mit Brotscheiben in einer Reihe mit immer weniger Scheiben.“ [Ex. zeigt auf die Reihe von Brotscheiben unten auf dem Blatt.] „Dieser Stapel mit Brotscheiben gehört irgendwo in die Reihe hinein. " [Ex. zeigt auf die Brotscheiben in dem Kasten oben links auf dem Blatt.] „Zeige die Stelle, wo dieser Stapel in der Reihe hineingehört. “ Da hierzulande solche Brotscheiben nicht üblich sind und die 
Kinder durch den Text darum verwirrt werden, ist das Resultat von 6\% Richtigen bei den Kleinen nicht verwunderlich. Es gab zudem Kinder, die die Sachlage richtig erkannt haben, es aber nicht wagten den Stapel in die Reihe hineinzuzwängen; es war ja gar kein Platz vorhanden. Interessanterweise zeigten viele Kinder auf den Zweierstapel oder auf den Viererstapel. Diese Kinder wollten die Brotscheiben vermutlich auf einen Stapel legen; und dabei wollten sie, dass das Hell-dunkel-Muster erhalten bleibt (1. KgJ 6\%; 2. $\mathrm{KgJ}$ 27\%; Gesamt 17\%).

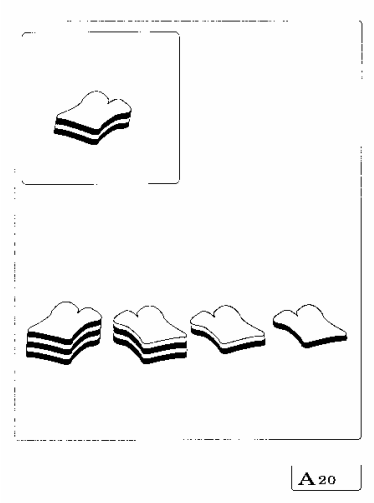

Abb. 36, Aufgabe A20

Der Umgang mit einzelnen und geordneten Gegenständen bildet die Grundlage der Komponente Einordnen in Reihenfolgen. Das Beherrschen der Tätigkeiten Einordnen von groß nach klein, von dick nach dünn, usw. bildet die Basis für den Ordinalzahlbegriff. Tatsächlich ist ein großer Teil der Kinder in der Lage, einfache Anordnungsanforderungen korrekt zu bewältigen. Anspruchsvollere Aufgaben lösen aber nur noch weit weniger als 50\% der Kinder. Diese Ergebnisse lassen auch hier wieder den Schluss zu, dass ein kleiner Teil der Kinder Aufgaben des Typs Ordinalzahl bewältigt.

\section{Kompetenzdifferenz bei Zahlwörtern zwischen dem ersten und dem zweiten Kin- dergartenjahr}

Auf den Unterschied in der mathematischen Kompetenz zwischen den Kindern, die den Kindergarten im ersten Jahr besuchen und denjenigen, die den Kindergarten im zweiten Jahr besuchen, habe ich bereits hingewiesen. In der folgenden Abbildung ist die Differenz zwischen diesen beiden Gruppen innerhalb der acht Komponenten nochmals abgebildet. 


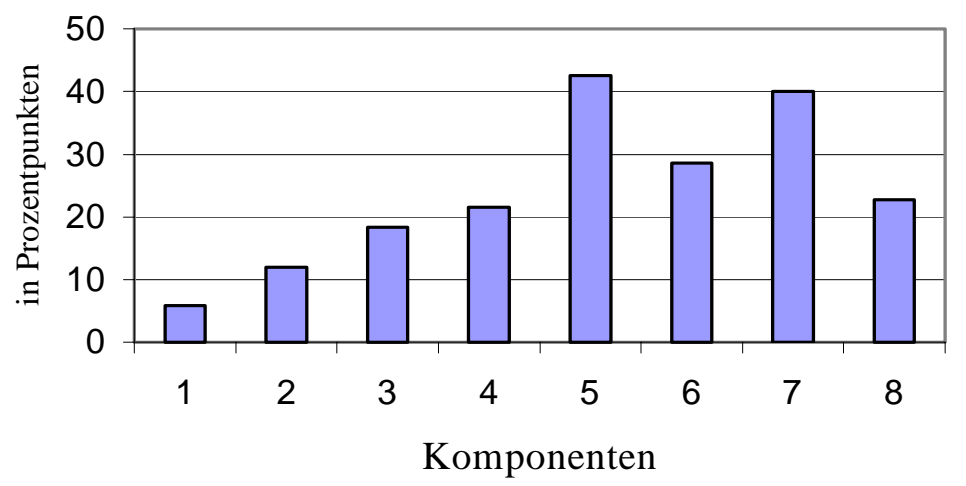

Abb. 37 Die Leistungsdifferenzen zwischen den Kindern im 1. Kindergartenjahr und den Kindern im 2. Kindergartenjahr in Prozentpunkten bezogen auf die acht Komponenten.

Die Darstellung zeigt die Durchschnitte aller Differenzen der jeweiligen fünf Aufgaben. Man sieht deutlich den rasanten Anstieg der Differenzen bis zur Komponente 5: „Gebrauch von Zahlwörtern“. Die durchschnittliche Differenz beträgt hier knapp 43 Prozentpunkte. Bei den folgenden Komponenten verbleibt diese Differenz auf sehr hohem Niveau bestehen. Warum ist die Differenz ab der Komponente 5 derart hoch, weit höher als in den vorigen Komponenten? Der Schluss liegt nahe und ist völlig lapidar: Die kleinen Kinder können weniger gut zählen.

\section{Gebrauch von Zahlwörtern}

\begin{tabular}{|c|c|c|c|}
\hline AufgabeNr & 1. Jahr & 2. Jahr & Alle \\
\hline \hline 21 & 27 & 76 & 53 \\
\hline 22 & 24 & 76 & 51 \\
\hline 23 & 18 & 70 & 46 \\
\hline 24 & 6 & 41 & 24 \\
\hline 25 & 6 & 32 & 20 \\
\hline
\end{tabular}

Tab. 11 Die Komponente „Gebrauch von Zahlwörtern“ ist für die Kleinen zu schwer. 


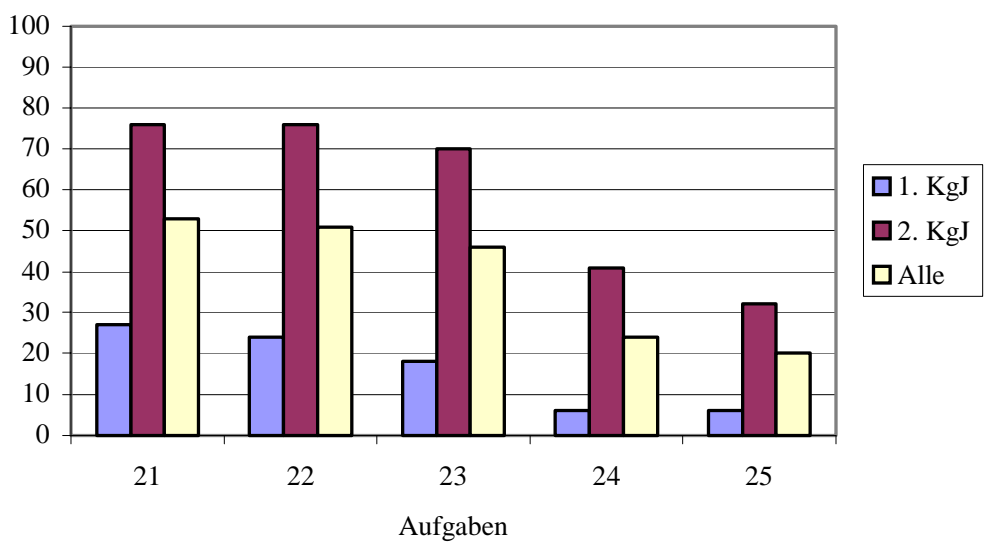

Abb. 38 Das Leistungsgefälle kommt deutlich zum Ausdruck.

Schon bei der ersten Aufgabe A21: „Zähle bis zwanzig“ klafft das Kompetenzniveau zwischen den Kindern aus dem ersten Kindergartenjahr und denjenigen aus dem zweiten Kindergartenjahr mit 49 Prozentpunkten weit auseinander. Es ist bemerkenswert, dass 76\% aller Kinder am Anfang des 2. Kindergartenjahres diese Aufgabe bewältigen. Das Zählen ohne Bezug auf reale Dinge ist aber offenbar für die Kinder im ersten Kindergarten sehr schwer. Einige Kinder weigerten sich weiter als bis $10 \mathrm{zu}$ zählen oder andere wirkten unmotiviert und ließen einzelne Zahlen zwischen 10 und 20 aus. Außerdem gab es ein Mädchen, das das Wort „Zwanzig“ nicht in ihrem Wortschatz verfügbar hatte und darum nur bis 19 zählen konnte. Wie schon einmal darauf hingewiesen, haben wir Kinder beobachtet, die über keine hohe ,abstrakte“ Zählperformanz verfügten; sobald aber konkrete Dinge abzuzählen waren, erhöhte sich diese Performanz erheblich und diese Kinder zählten dann ohne Mühe bis 20 (siehe Kapitel 9). (1. KgJ 27\%; 2. KgJ 76\%; Gesamt $53 \%$ )

Noch höher liegt die Differenz der Ergebnisse der beiden Kindergruppen bei der Aufgabe A22: [Ex. zeigt dem Kind ein Bild.] „Zeige auf den Kasten mit sieben Punkten. “ mit 52 Prozentpunkten. Diese Ergebnisdifferenz der Kinder aus dem 1. KgJ und dem 2. KgJ sind bei der Aufgabe A22 zusammen mit der Aufgabe A23 von allen 40 Aufgaben am größten. Auch hier liegen die älteren Kinder wieder mit 76\% sehr hoch. Einige Kinder erfassten mit einem Blick das Sechsermuster und erkannten sofort, dass der eine Punkt dazu sieben ergibt (1. KgJ 24\%; 2. KgJ 76\%; Gesamt 51\%). 


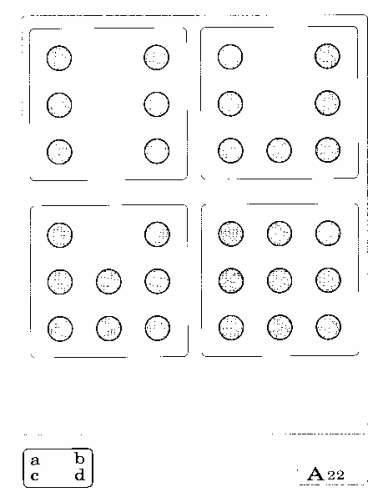

Abb. 39, Aufgabe A22

Die Aufgabe A23: „Zähle weiter von neun bis fünfzehn: sechs, sieben, acht ... “ haben etliche Kinder aus dem ersten Kindergartenjahr überhaupt nicht verstanden. Für sie beginnt das Zählen eigentlich immer bei der Zahl Eins und sie bekundeten dann Mühe, bei der Acht einzusteigen, während wiederum andere frisch weiter als bis und mit 15 zählten (1. KgJ 18\%; 2. KgJ 70\%; Gesamt 46\%).

Die Aufgabe A24: [Ex. zeigt dem Kind das Bild.] „Zeige auf die achtzehnte Blume. “ ist außerordentlich schwer und für die jüngeren Kinder nicht bewältigbar. Die Blumen sind auch sehr nah beieinander gezeichnet, sodass das Zeigen mit den Fingern auf die Blumen erschwert wird und es darum zu Verschiebungen kam. ${ }^{32}$ Es gab einige Kinder, die den Begriff „die 18. Blume“ nicht verstanden; sie zählten zwar die Blumen ab, stoppten aber nicht bei der 18. Blume (1. KgJ 6\%; 2. KgJ 41\%; Gesamt 24\%).

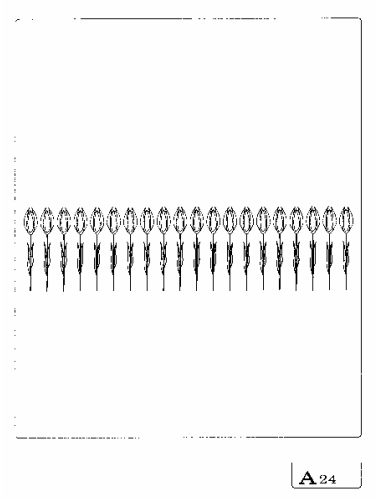

Abb. 40, Aufgabe A24

\footnotetext{
${ }^{32}$ Hätte man die Blumen mit einem didaktisch begründet Ansatz aufgereiht (die Kraft der Fünf) und nach jeweils 5 Blumen eine kleine Lücke gelassen, wäre das Auffinden der 18. Blume bestimmt einfacher gewesen.
} 
Ebenso ist die Aufgabe A25: „Zähle bis vierzehn und überspringe immer jeweils eine Zahl: zwei, vier, sechs ... „, für Kindergartenanfängerinnen und -anfänger zu anspruchsvoll und in diesem Alter noch nicht lösbar. Die allermeisten Kleinen haben auch hier die Aufgabe eigentlich gar nicht richtig verstanden. Andere bemühten sich redlich, jeweils eine Zahl auszulassen, schafften es aber meistens nicht; die Macht der regulären Zahlreihe ist offenbar zu groß. Viele aus dem 2. KgJ haben nach der sechs mit sieben, acht, neun, ... weitergezählt oder andere zählten auch bei sieben weiter, ließen dann aber immerhin jeweils eine Zahl aus: sieben, neun, elf, ... (1. KgJ 6\%; 2. KgJ 32\%; Gesamt $20 \%)$.

Auffallend und aufschlussreich ist die Erkenntnis, dass sich die Kinder während des ersten Kindergartenjahres die Kompetenz des Zählens aneignen. Nach den vorliegenden Ergebnissen kann man schließen, dass der Gebrauch von Zahlwörtern von einem Grossteil der Kinder aus dem 1. Kindergarten noch nicht gefestigt ist; viele verstehen den Sinn des Zählens nicht umfassend. Die Zahlwortreihe ist für einige Kinder ein Instrument, zwar fest eingeübt, oft aufgesagt und selten reflektiert, um nur kleine Mächtigkeiten abzuzählen, für komplexere Aufgaben ist ihr Instrument noch nicht brauchbar. Die um ein Jahr älteren Kinder aus dem 2. KgJ haben den Jüngeren gegenüber einen erheblichen Vorsprung, d.h.: Während diesem Jahr nehmen die Kompetenzen im Gebrauch von Zahlwortreihen deutlich zu und damit ist die Lehrplanforderung „Zählen von 1 bis 10“ im neuen Lehrplan für Kindergarten im Kanton Basellandschaft gut begründet und abgestützt.

\section{Strukturiertes Zählen}

\begin{tabular}{|c|c|c|c|}
\hline AufgabeNr & 1. Jahr & 2. Jahr & Alle \\
\hline \hline 26 & 36 & 70 & 54 \\
\hline 27 & 52 & 65 & 59 \\
\hline 28 & 18 & 57 & 39 \\
\hline 29 & 18 & 54 & 37 \\
\hline 30 & 3 & 24 & 14 \\
\hline
\end{tabular}

Tab. 12 Die Resultate liegen bei Kindern im 2. KgJ beachtlich hoch. 


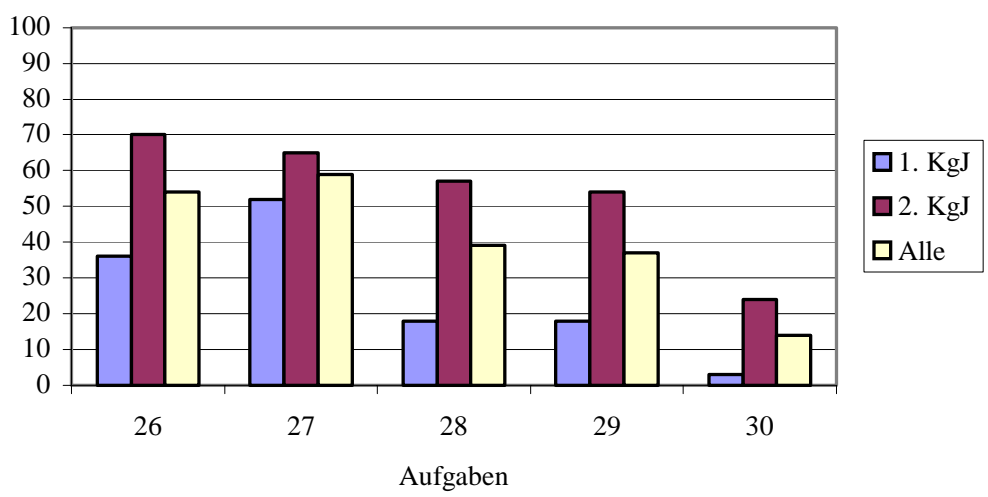

Abb. 41 Man muss sich immer in Erinnerung rufen, dass es sich um Kindergartenkinder handelt!

Mit Ausnahme der letzten Aufgabe A30 liegen die Ergebnisse der Kinder im zweiten Kindergartenjahr zum Teil deutlich über 50\%. Die Kinder im ersten Kindergartenjahr schaffen die 50\% nur einmal bei der Aufgabe A27 mit 52\%. Die Resultate liegen zwischen 52\% bei Aufgabe A27 und 3\% bei der Aufgabe A30. Die Differenz über alle Aufgaben in dieser sechsten Komponente liegt dann auch bei hohen 29\% (Siehe Abb. 37, Seite 119). Im Unterschied zum Beispiel zur Aufgabe A24: „Zeige auf die achtzehnte Blume" ist die Erfassung einer Menge von Elementen für die Kinder besser bewältigbar. Mehr als die Hälfte aller Kinder schaffte die Antwort auf die Aufgabe A26: [Ex. legt 16 Holzwürfel in vier Reihen von jeweils vier Holzwürfel mit etwas Abstand zwischen den Holzwürfeln auf den Tisch.] „Zeige auf die Holzwürfel und zähle sie.“ Die Anzahl der Holzwürfel ist durch die Strukturierung in 4 Reihen offenbar leicht zu bestimmen. Diese Situation nutzten aber wenige Kinder direkt; die meisten zeigten mit den Fingern auf jedes zu zählende Objekt. Eine große Anzahl von Kindern schob zudem die gezählten Würfel beiseite und erreichte dadurch eine hohe Sicherheit in der Bestimmung der Anzahl der Würfel. Dass dann beim Zählprozess die letzte Zählzahl als Kardinalzahl benutzt werden kann, ist für die Kinder selbstverständlich. Solange die zu zählende Menge nicht über 10 reicht, halten die jüngeren Kinder mit den älteren mit (1. KgJ 36\%; 2. KgJ 70\%; Gesamt 54\%). 


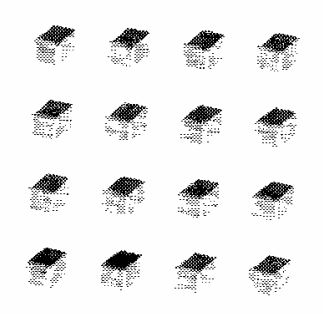

Abb. 42, Aufgabe A26

Die Aufgabe A27: [Ex. legt neun Holzwürfel in einem Kreis mit etwas Abstand zwischen den Holzwürfeln auf dem Tisch.] „Zähle diese Holzwürfel. [Das Kind darf auf die Holzwürfel zeigen oder sie während des Zählens zur Seite schieben.] Fast alle Kinder, die die Holzwürfel zur Seite geschoben haben, kamen auf das richtige Resultat. Eine hohe Erfolgsquote erreichten die Kinder, die den ersten gezählten Würfel mit einem Finger berührten und dann während des gesamten Zählprozesses nicht mehr losließen. Diejenigen Kinder, die nur auf die Würfel zeigten, zählten aus nahe liegenden Gründen meistens eins zuwenig oder eins zuviel (1. KgJ 52\%; 2. KgJ 65\%; Gesamt 59\%).

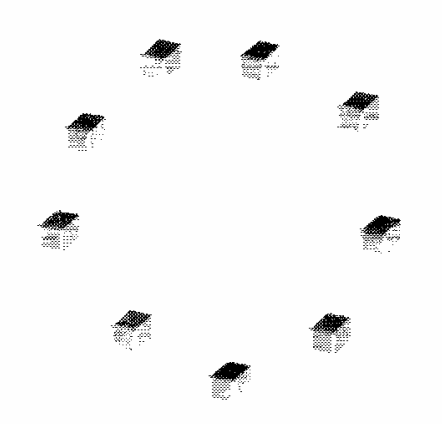

Abb. 43, Aufgabe A27

Aufgabe A28: [Ex. legt 20 Holzwürfel in einem Haufen mit etwas Abstand zwischen den Holzwürfeln auf den Tisch.] „Zähle diese Holzwürfel.“ [Das Kind darf auf die Holzwürfel zeigen oder sie während des Zählens zur Seite schieben.] Alle Kinder, die bei den vorherigen Aufgaben die zu zählenden Würfel berührten oder verschoben, wendeten diese Technik auch bei dieser Aufgabe an. Trotzdem gab es einen heftigen Leistungseinbruch vor allem bei den Kindern aus dem ersten Kindergartenjahr. Hier macht sich deutlich die noch mangelnde Zählkompetenz bemerkbar; die Kollektion von Würfeln ist mit 20 für viele kleine Kinder unübersichtlich. Obwohl sie die gezählten Würfel auf die Seite schoben, kam es sehr oft vor, dass sie diese schon gezählten Würfel noch- 
mals zählten oder noch nicht gezählte als gezählt betrachteten (1. KgJ 18\%; 2. KgJ 57\%; Gesamt 39\%).

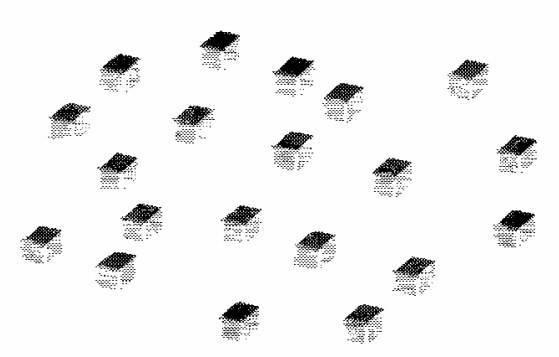

Abb. 44, Aufgabe A28

Die besagte Kompetenzdifferenz zwischen den beiden Altersstufen zeigt sich auch deutlich mit 36 Prozentpunkten bei der Aufgabe A29: „Ich gebe dir ein Bild, das du kurz anschauen sollst." [Ex. zeigt dem Kind das Bild für 2 Sekunden und nimmt das Bild weg.] „Wie viele Punkte waren es zusammen?“ [Die Antwort „vier und fünf“ reicht aus.] Viele ältere Kinder beherrschten offenbar die simultane Anzahlerfassung und nannten das Resultat relativ schnell. Einige Kinder haben sich offenbar ein mentales Bild der beiden Würfelbilder geschaffen und zählten dann die Würfelaugen vor ihrem geistigen Auge ab. Die Kleinen haben diese Fähigkeit der simultanen Anzahlerfassung noch nicht erlangt und sind auf dieses Zählen angewiesen.

Ein Kind hat die Augenzahlen wie beschrieben gezählt, hielt dann inne und erinnerte sich dann nochmals an die zwei Würfelbilder und erkannte aus der Situation, dass das abgezählte Resultat auch einfacher durch eine Summenbildung zu erreichen gewesen wäre: „Neun ist ja vier und fünf.“ Das Kind hat in diesem Moment die Addition entdeckt und begriffen und freute sich unsäglich. Viele Kinder aus dem ersten Kindergartenjahr benutzten oft ihre Finger, um die Anzahl der Punkte zu eruieren und einige von ihnen nannten dann auch 4 und 5, hingegen waren sie außerstande, die Summe zu nennen. $18 \%$ schafften das richtige Resultat (Die Antwort „4 und 5“ ist richtig). Ich habe zudem beobachtet, dass diese neue Art der Fragestellung (kurz zeigen - verstecken fragen) einen nicht zu vernachlässigen Überraschungseffekt aufweist und von der eigentlichen Aufgabe ablenken kann (1. KgJ 18\%; 2. KgJ 54\%; Gesamt 37\%). 


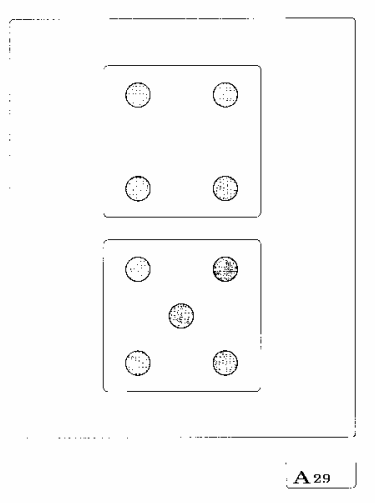

Abb. 45, Aufgabe A29

Die am schlechtesten gelöste Aufgabe (Siehe Abb. 12 Seite 103) überhaupt war die Aufgabe A30: [Ex. legt 17 Holzwürfel auf den Tisch in einer Reihe mit etwas Abstand zwischen den Holzwürfeln.] „Hier siehst du siebzehn Holzwürfel. Zeige auf die Holzwürfel und zähle sie rückwärts. “ Nur 3\% der Kleinen und nur 24\% der Älteren schafften diese Aufgabe richtig, was den tiefen Durchschnitt aller von 14\% ergab.

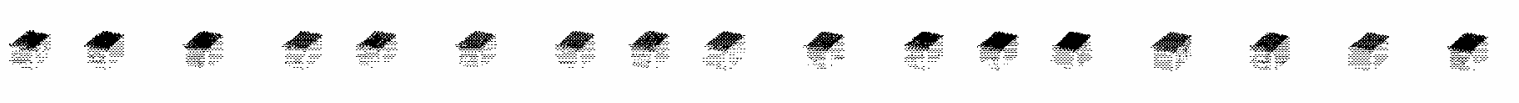

Abb. 46, Aufgabe A30

Rückwärtszählen ist sehr schwierig, weil der lebensweltliche Kontext fehlt; Kinder zählen eher selten oder sogar nie rückwärts. Darum ist nie eine klare Sprechvorlage gelegt worden wie beim Vorwärtszählen. Wo das Vorwärtszählen wie aus einem Guss läuft, oft gehört und aufgesagt, zuerst noch ohne Vorstellung vom Zahlbegriff, einfach als Wortspiel (siehe Prinzipien-Nachher-Theorie Seite 50ff), passiert solches nie oder zumindest äußerst selten beim Rückwärtszählen. Viele Kinder kannten zudem den Begriff des Rückwärtszählens nicht und zählten sehr unsicher vorwärts, da sie wussten, dass das nicht stimmen konnte. Andere lösten die Aufgabe des Rückwärtszählens, indem sie statt von links nach rechts einfach am rechten Ende begannen und von rechts nach links vorwärts zählten (1. KgJ 3\%; 2. KgJ 24\%; Gesamt 14\%).

In dieser Komponente Strukturiertes Zählen wie auch in der vorigen Komponente Gebrauch von Zahlwörtern wird klar dokumentiert, dass die fünf Phasen der Prinzipien- 
Nachher-Theorie (siehe Seite $50 \mathrm{ff})^{33}$ erst bei den älteren Kindern vollständig erfolgreich durchlaufen worden sind. Es gibt Kinder aus dem ersten wie auch aus dem zweiten Kindergartenjahr, die kaum die Zahlwortreihe beherrschen, die also die erste Phase noch nicht erfolgreich durchlaufen haben. Aufmerksam muss man werden, wenn es solche Kinder gibt, die mit den erfolgreicheren bei Schuleintritt im selben Klassenverband unterrichtet werden müssen. Die schon angesprochenen (vorläufigen) Ergebnisse von M. Grüssing (2002) weisen darauf hin, dass schlechte „Startpositionen“ nur schwer wieder gutzumachen sind.

\section{Ergebnisbezogenes Zählen}

\begin{tabular}{|c|c|c|c|}
\hline AufgabeNr & 1. Jahr & 2. Jahr & Alle \\
\hline \hline 31 & 33 & 57 & 46 \\
\hline 32 & 15 & 43 & 30 \\
\hline 33 & 30 & 49 & 40 \\
\hline 34 & 6 & 41 & 24 \\
\hline 35 & 6 & 30 & 19 \\
\hline
\end{tabular}

Tab. 13 Viele Aufgaben der Komponente „Ergebnisbezogenes Zählen“ sind für die Kleinen zu schwer.

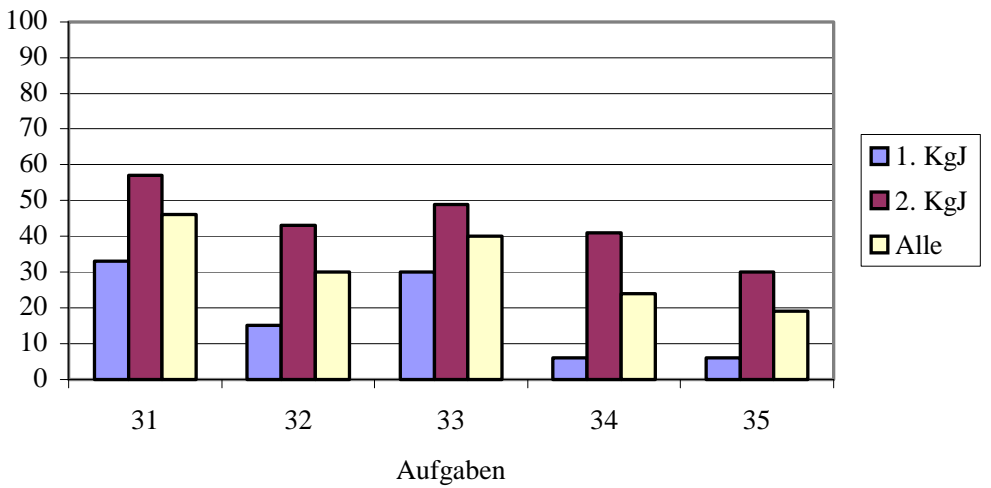

Abb. 47 Das ergebnisbezogene Zählen erfordert einen gefestigten Zahlbegriff.

${ }^{33}$ Zur Erinnerung:

1. Phase der noch nicht differenzierten Ganzheitsauffassung der Zahlenfolge

2. Phase der differenzierten Ganzheitsauffassung der Zahlwortreihe

3. Phase des Weiterzählens-Könnens

4. Phase der Auffassung der Zahlwörter als zählbare Einheiten

5. Phase der „,zweiseitigen Durchlaufbarkeit“ der Zahlwortreihe 
Im Gegensatz zur vorher vorgestellten Komponente darf hier nicht auf die zu zählenden Objekte gezeigt werden. Dieser Umstand erschwert die Aufgaben natürlich erheblich. Wohl aus diesem Grund bereitete diese Komponente den Kindern die meisten Probleme (Siehe Seite 101, Abb. 10, Seite 102, Tab. 6 sowie Seite 103, Abb. 12).

Bescheiden ist aber trotzdem das Abschneiden bei der Aufgabe A31: [Ex. gibt dem Kind 15 Holzwürfel.] „Lege eine Reihe von elf Holzwürfeln. “ Nur jeder dritte aus dem ersten Kindergartenjahr und 57\% der Kinder aus dem zweiten Kindergartenjahr konnten die elf Holzwürfel legen. Einige Kinder suchten sich zuerst die 11 Holzwürfel aus dem Haufen und legten dann erst die Reihe, während andere direkt die Reihe legten und dann aber oft mehr als 11 Würfel legten. Gesamthaft haben $46 \%$ diese Aufgabe geschafft (1. KgJ $33 \%$; 2. KgJ 57\%; Gesamt 46\%).

Aufgabe A32: [Ex. legt 20 Holzwürfel in einer Reihe auf den Tisch mit etwas Abstand zwischen den Holzwürfeln.] „,Wie viele Holzwürfel liegen hier? “ [Das Kind darf nicht auf die Holzwürfel zeigen.] Dies ist eine schwierige Aufgabe. Wie schnell hat man sich um eins verzählt; man zählt einen Holzwürfel zweimal oder vergisst einen. Beachtlich ist darum, dass fast jedes dritte Kind diese Aufgabe schaffte. Viele Kinder aus dem ersten Kindergartenjahr versuchten entgegen der Anordnung mit den Fingern die Holzwürfel zu berühren und mussten darum beim Zählvorgang unterbrochen werden $(1 \mathrm{KgJ}$ 15\%; 2. KgJ 43\%; Gesamt 30\%).

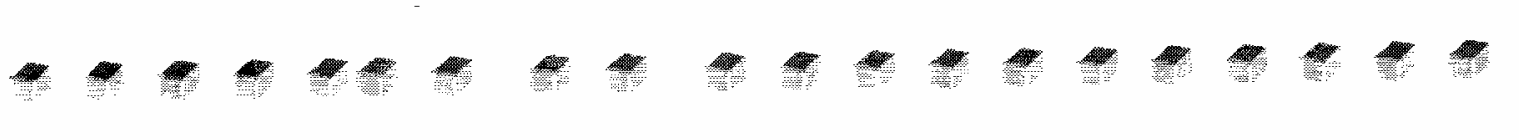

Abb. 48, Aufgabe A32

Aufgabe A33: [Ex. legt 15 Holzwürfel auf den Tisch in drei Reihen mit jeweils fünf Holzwürfeln und mit etwas Abstand zwischen den Holzwürfeln.] ,, Wie viele Holzwürfel liegen hier?" [Das Kind darf nicht auf die Holzwürfel zeigen.] Diese drei Reihen machen die gesamte Menge übersichtlicher als die 20 Holzwürfel in einer einzigen Reihe bei Aufgabe A32 (Man vergleiche mit der Aufgabe A26 auf der Seite 124). Darum wohl erreichen hier die Kinder im ersten Kindergartenjahr beachtliche 30\%. Da das Antippen 
oder das Herumschieben der Holzwürfel verboten war, haben einige ältere Kinder versucht, die Kollektion mit Rechnen zu bestimmen (1. KgJ 30\%; 2. KgJ 49\%; Gesamt $40 \%)$.

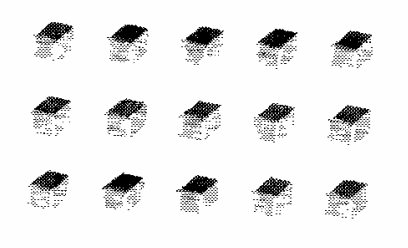

Abb. 49, Aufgabe A33

Dass das Ergebnis der Aufgabe A34: [Ex. legt 19 Holzwürfel in einem Haufen auf den Tisch mit etwas Abstand zwischen den Holzwürfeln.] „Wie viele Holzwürfel liegen hier?" [Das Kind darf nicht auf die Holzwürfel zeigen.] mit 24\% tief liegt, erstaunt nicht, da es sich um eine sehr schwere Aufgabe handelt. Es muss aber hervorgehoben werden, dass doch $41 \%$ der Kinder im zweiten Kindergartenjahr diesen wirren Haufen von 19 Holzwürfeln ohne auf die einzelnen Objekte zu zeigen, korrekt abgezählt haben und dies ein knappes Jahr vor Schuleintritt. Der Niveauunterschied zwischen den beiden Altersgruppen ist mit 35 Prozentpunkten hoch. Für die Jüngeren ist diese Aufgabe zu schwer (1. KgJ 6\%; 2. KgJ 41\%; Gesamt 24\%).

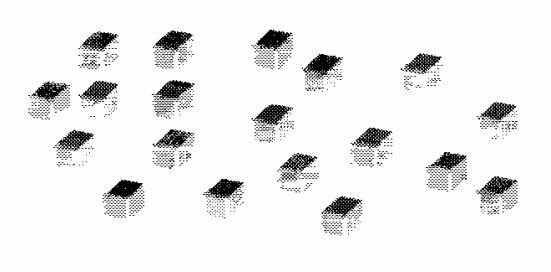

Abb. 50, Aufgabe A34

Die Aufgabe A35: [Ex. legt 5 Holzwürfel auf den Tisch.] „Hier sind fünf Holzwürfel. Ich schiebe sie unter meine Hand. " [Ex. schiebt die Holzwürfel unter seine Hand. Dann schiebt er 7 weitere Holzwürfel, die er dem Kind zeigt, unter seine Hand.] „Ich füge sieben Holzwürfel dazu. Wie viele Holzwürfel habe ich jetzt unter meiner Hand?" war für die jüngeren Kinder ebenfalls zu anspruchsvoll. Auch hier schafften nur 6\% die richtige Antwort. Von den Älteren bewältigten immerhin 30\% diese Aufgabe. Es bleibt aber zu bedenken, dass einerseits die Aufgabenstellung andersgeartet ist als die übrigen, und andererseits, dass eine sehr komplexe Rechnung (mit Zehnerübergang) abstrakt vorge- 
legt wurde. Die Kinder mussten sich die Situation im Nachhinein selbst wieder zurechtlegen. Sie mussten sich an die ersten fünf Holzwürfel erinnern, dann an die sieben daruntergeschobenen. Was passiert da unter der Hand? 19\% aller Kinder hatten die Situation vollständig im Griff und lieferten das richtige Resultat (1. KgJ 6\%; 2. KgJ 30\%; Gesamt 19\%).

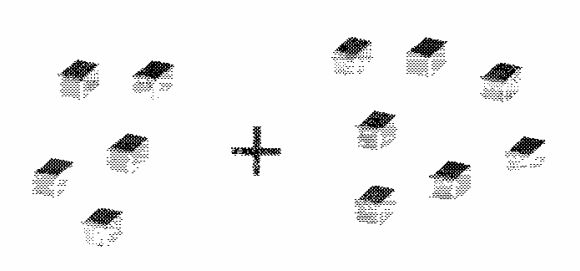

Abb. 51, Aufgabe A35

In dieser Komponente Ergebnisbezogenes Zählen muss deutlich zwischen den Kindern aus dem ersten und den Kindern aus dem zweiten Kindergartenjahr unterschieden werden. Während für die Jüngeren diese Komponente deutlich zu schwer war, konnte bei den älteren Kindern beobachtet werden, dass neben der höheren Zählkompetenz auch die Konzentrationsfähigkeit die bessere war. Etliche der jüngeren Kinder schweiften bei Aufgaben geistig ab, vor allem wenn darauf beharrt wurden musste, die Holzwürfelchen beim Zählen nicht zu berühren; das war einfach zu anstrengend.

\section{Allgemeines Zahlenwissen}

\begin{tabular}{|c|c|c|c|}
\hline AufgabeNr & 1. Jahr & \multicolumn{1}{|l|}{ 2. Jahr } & Alle \\
\hline \hline 36 & 52 & 73 & 63 \\
\hline 37 & 42 & 68 & 56 \\
\hline 38 & 27 & 46 & 37 \\
\hline 39 & 39 & 57 & 49 \\
\hline 40 & 6 & 41 & 26 \\
\hline
\end{tabular}

Tab. 15: Hoffentlich wird diesem erstaunlichen Resultat beim Schuleintritt Rechnung getragen! 


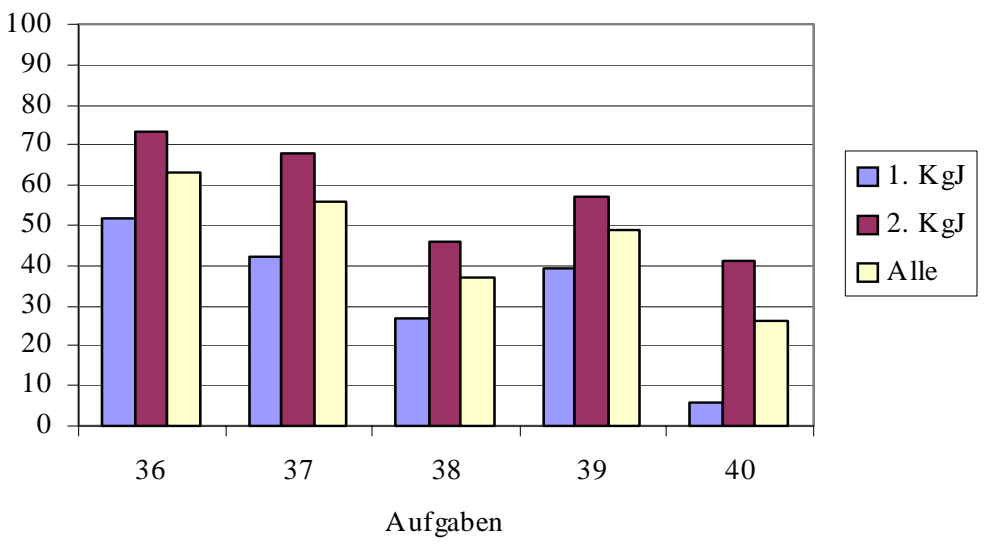

Abb. 52 Die letzte Komponente „Allgemeines Zahlenwissen“.

Aufgabe A36: „Hier siehst du zwei Kisten. “ [Ex. zeigt auf die Kisten in dem Bild.] „Es sind neun Bonbons in der schwarzen Kiste. In der weißen Kiste sind dreizehn Bonbons. In welcher Kiste sind die meisten Bonbons? “ Kinder begreifen schon früh, dass dreizehn mehr ist als neun. Dreizehn hat ja die erste Hürde der Zehn schon überschritten. Außerdem geht es bei dieser Aufgabe darum, gut zuzuhören und sich nicht von der dominanteren schwarzen Schachtel beeinflussen zu lassen (1. KgJ 52\%; 2. KgJ 73\%; Gesamt $63 \%)$.

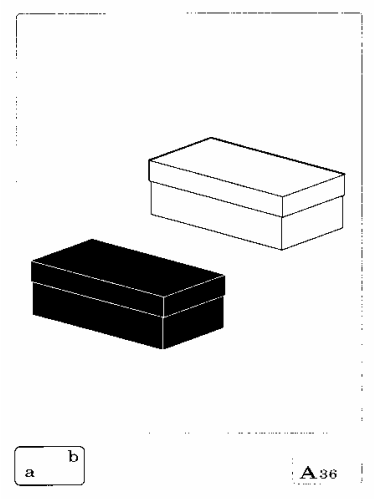

Abb. 53, Aufgabe A36

Aufgabe A37: [Ex. zeigt auf das Bild mit 9 Murmeln.] „Du hast neun Murmeln. Du verlierst drei Murmeln. Wie viele Murmeln hast du übrig? Zeige auf den Kasten mit der richtigen Anzahl von Murmeln." [Ex. zeigt auf die Reihe der Bilder unten auf dem Blatt.] Diese Subtraktionsaufgabe bewältigten 56\% aller Kinder. Die Anordnung der Murmeln in zwei Reihen zu je drei Murmeln im richtigen Resultat ist die gleiche wie in der Ausgangsstellung. Dieser Umstand kann für die Lösung sehr hilfreich sein. War ein 
Kind unsicher, zeigte es darum auf das richtige Bild, eben weil es der Ausgangssituation ähnlich ist (1. KgJ 42\%; 2. KgJ 68\%; Gesamt 56\%).

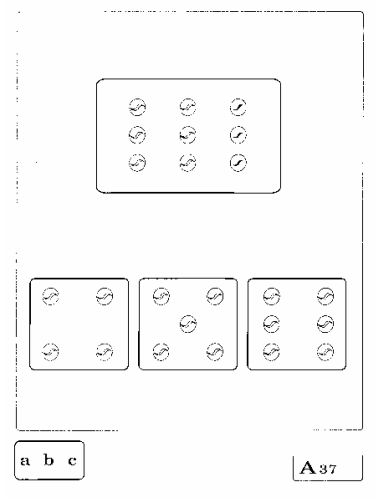

Abb. 54, Aufgabe A37

Die Aufgabe A38: [Ex. zeigt auf das Bild mit den 8 Hühnern.] „Ein Bauer hat acht Hühner. Er kauft noch zwei Hühner. " [Ex. zeigt auf das Bild mit den zwei Hühnern.] „Wie viele Hühner hat der Bauer jetzt? Zeige auf den Kasten mit der richtigen Anzahl von Hühnern. “ [Ex. zeigt auf die Reihe von Bildern unten auf dem Blatt.] bereitete den Kindern zwei Probleme. Zum ersten ist die sprachliche und inhaltliche Situation für die Kinder meines Erachtens äußerst kompliziert. Der Sinn der ganzen Aufgabe muss von den Kindern erst erschlossen werden und dafür braucht es ein ganz erhebliches Maß an Vorstellungsvermögen. Das zweite Problem ist die rechnerische Bewältigung der Aufgabe. Ein Viertel der Kleineren und knapp die Hälfte der Älteren schafften diese Aufgabe. Im Schnitt waren es 37\%, das ist ein erstaunlich hohes Ergebnis (1. KgJ 27\%; 2. KgJ 46\%; Gesamt 37\%).

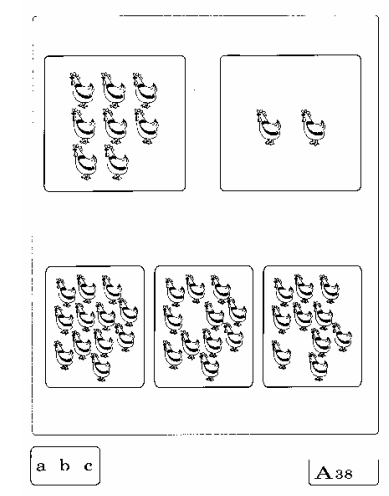

Abb. 55, Aufgabe A38 
Aufgabe A39: „Hier siehst du ein Gebäude mit Fenstern und Türen. “ [Ex. zeigt auf die Fenster und Türen in dem Gebäude, aber nicht auf jede(s) einzeln.] „Bäume stehen auch vor dem Gebäude. Kannst du zählen wie viele Fenster das Gebäude hat?" Diese Aufgabe stellt eigentlich hohe Anforderungen an kontextgebundenem Vorstellungsvermögen. Fast die Hälfte der Kinder besitzt vielleicht dieses Vorstellungsvermögen. Es ist hier aber zu bemerken, dass die Darstellung nicht sehr glücklich ist; wir haben oft beobachtet, dass viele Kinder nicht die Fenster, sondern die sichtbaren Türen zählten und so auf die Anzahl Fenster schlossen (1. KgJ 39\%; 2. KgJ 57\%; Gesamt 49\%).

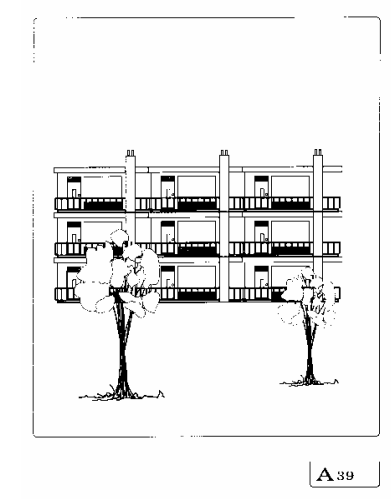

Abb. 56, Aufgabe A39

Die letzte Aufgabe schließt das ganze Interview ab. A40: „Du hast zweimal gewürfelt.“ [Ex. zeigt auf die beiden Würfelbilder.] „Welche Zahl hast du insgesamt gewürfelt und wohin darf die Figur gehen?" Ausgehend von den Würfelbildern muss das Kind die richtigen Zahlen erkennen, diese nicht ganz einfache Addition durchführen und dann das Resultat auf dem Blatt zeigen. All das im Kopf durchzuführen ist für kleine Kinder nicht einfach und zudem sind seit Beginn des Interviews meistens schon 20 bis 30 Minuten vergangen und die meisten Kinder sind sehr müde und die Konzentrationsfähigkeit ließ stark nach. Den wenigsten Kindern gelang es, die Würfelpunkte direkt zusammenzuzählen und das Ergebnis zu zeigen. Die meisten Kinder zählten den ersten Würfel ab und dann den zweiten. So schaffte jedes 4. Kind diese schwere Aufgabe, wobei zu beachten ist, dass die Kinder im ersten Kindergartenjahr die Aufgabe nicht bewältigten (1. KgJ $6 \%$; $2 . \mathrm{KgJ} 41 \%$; Gesamt 26\%). 


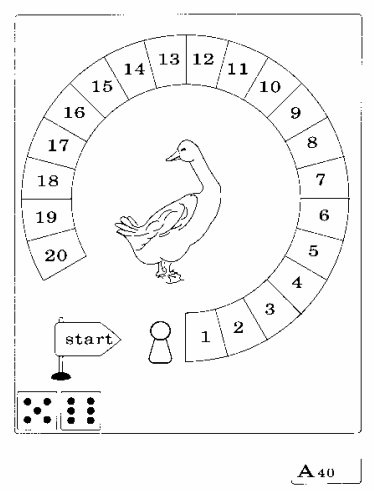

Abb. 57, Aufgabe A40

Bei der Durchsicht aller dieser Aufgaben wird deutlich, was die Kindergartenkinder schon zu leisten imstande sind. Nicht nur die hohe mathematische Kompetenz ist erstaunlich, sondern auch die Konzentrationsfähigkeit über die doch lange Zeit von 20 bis 30 Minuten. Durch Einzelinterviews erhalten die Kinder die volle Hinwendung der Interviewerin oder des Interviewers. Das ist hilfreich für die Beibehaltung einer hohen Aufmerksamkeit über eine längere Zeit. Vor allem bei den Aufgaben 21 bis 40 konnte die Prinzipien-Nachher-Theorie vielfach sehr schön nachgeprüft werden. Im nächstfolgenden Kapitel 8 werde ich genauer darauf eingehen. 


\section{Auswertung der Ergebnisse}

\subsection{Vergleich der Ergebnisse NL - D - CH}

Allen in den internationalen Kompetenzvergleichen wie TIMMS und PISA untersuchten Kindern und Jugendlichen ist gemeinsam, dass sie alle einmal mit der ersten Grundschuleklasse begonnen haben. Wenn unsere These, dass gute Startbedingungen beste Voraussetzungen für eine erfolgreiche Schulkarriere sind, Gültigkeit hat, ist es von Interesse, zu wissen, wo denn unsere Schulanfängerinnen und Schulanfänger im internationalen Vergleich etwa stehen. Wie wir gesehen haben, werden im Kindergarten u.a. die ersten mathematischen Begriffe gebildet und eine solide Zählkompetenz geschaffen, d.h. die Startbedingungen bei Eintritt in die Grundschule werden im Kindergarten gelegt. Wir sind nun in der Lage, Testergebnisse von Kindern aus den drei Nationen Deutschland, Holland und der Schweiz gegenüberzustellen, wobei zu bemerken ist, dass erst eine Langzeitstudie unsere These klar beweisen könnte.

Um die Ergebnisse der Kinder in diesen drei Ländern beurteilen zu können, sind gute Kenntnisse der jeweiligen Bildungskonzeptionen der vier- bis siebenjährigen Kinder unentbehrlich. Dieser institutionalisierte Bildungsbeginn unterscheidet sich nämlich in den drei genannten Staaten beträchtlich. Bevor ich einige Resultate vergleiche, stelle ich kurz die Bildungseinrichtungen der vier- bis siebenjährigen Kinder in Holland, Deutschland und der Schweiz vor.

\subsubsection{Das holländische System}

Seit fast zwei Jahrzehnten gibt es in Holland keine Kindergärten mehr. Um einen Bruch zwischen den zwei Bildungseinrichtungen Kindergarten und Schule zu vermeiden, hat man den Kindergarten vor achtzehn Jahren vollständig in die Schule integriert. Diese 1985 neu entstandene Basisschule (basisonderwijs) umfasst acht Jahrgangsstufen oder genauer die Gruppen 1 bis 8, wobei die Gruppen 1 und 2 unseren Kindergärten entsprechen. Das neu entwickelte Unterrichtskonzept sieht vor, den Kindern individuelles, differenziertes und kontinuierliches Lernen zu ermöglichen. Es wird darauf geachtet, dass die Lernaktivitäten sich aus dem Spiel heraus entwickeln. Da zum Beispiel nicht alle Kinder mathematische Relationen von sich aus entdecken oder sich spontan dafür interessieren, wird darauf geachtet, schon in der Gruppe 1 und 2 Situationen und Aktivitäten 
anzubieten, die ein Bedürfnis nach mathematischen Fertigkeiten herausfordern. Die Lehrpersonen sind angehalten, vorhandenes Wissen und Kompetenzen der Kinder aufzunehmen und zu erweitern (vgl. Zopfi 1997).

\subsubsection{Das Deutsche System}

Auch in Deutschland unterscheiden sich die Kindergärten wesentlich von denen in der Schweiz. Vor allem das Eintrittsalter ist mit drei Jahren viel tiefer als in der Schweiz. Obwohl der Besuch des Kindergartens freiwillig ist, garantieren doch einige Bundesländer für jedes Kind einen Kindergartenplatz. Die Kindergärten in Deutschland sind meistens kommunale Einrichtungen oder Einrichtungen von freien Trägern. Solche freien Träger sind z. B. das Rote Kreuz, die Arbeiterwohlfahrt, die Evangelische oder Katholische Kirche, u.a.m. Der Kindergarten bietet die Möglichkeit, Kontakte zu knüpfen und zu pflegen. Hier werden die Jungen und Mädchen zusammen in altersgemischten Gruppen erzogen. Die meisten Kinder besuchen den Kindergarten nur am Vormittag. Manche Kinder bleiben aber auch bis zum Nachmittag im Kindergarten. Das letzte Kindergartenjahr vor der Einschulung in die Grundschule dient dann dazu, die Aufnahme und das Lernen in der Schule gezielt vorzubereiten. Es gibt aber keine spezifischen gesetzlichen Empfehlungen von landesweiter Gültigkeit. Richtlinien werden nur auf lokaler Ebene vorgegeben. In Deutschland gehören im Gegensatz zu fast allen europäischen Staaten mathematische Lernerfahrungen nicht zum Programm, weil keine expliziten schriftlichen Empfehlungen existieren. Es wird aber gleichwohl in vielen Kindergärten im letzten Jahr Mathematik in vielfältiger Art und Weise betrieben. Vor allem ist man in den letzten Jahren vermehrt dazu übergegangen, lebenspraktische Themen zu bearbeiten. Die Kinder werden in Deutschland zwischen sechs und sieben Jahren eingeschult.

\subsubsection{Das Schweizer System}

Im Allgemeinen besuchen die Kinder 2 Jahre lang den Kindergarten, wobei aber auch hier kein einheitliches Konzept existiert, da man nicht von einem schweizerischen Kindergartenkonzept sprechen kann. Da die Bildung in der Schweiz unter kantonaler Hoheit fällt, gibt es entsprechend den 26 Kantonen gesamthaft 26 Bildungssysteme. Ich beschränke mich darum wegen der Untersuchungsdaten auf die zwei Kantone Baselland und Luzern, wobei in der deutschen Schweiz die Rahmenlehrpläne der Kindergärten durchaus vergleichbar sind. Erst in den letzten vier bis fünf Jahren sind in verschiedenen Kantonen (St. Gallen, Bern, Solothurn, Baselland u.a.) Anstrengungen unternommen 
worden, auch der kognitiven Bildung vermehrt Beachtung zu schenken, und entsprechende Rahmenlehrpläne wurden entwickelt. In einigen Kantonen gibt es aber heute noch Richtlinien, die besagen, dass fachliche Förderung im Kindergarten zu unterlassen sei. Diese Ansicht wird nun aber in fast allen Kantonen fallengelassen. Es wird heute angestrebt, Sozialisation- und Bildungsfunktionen in die Vorschulerziehung mit einzubeziehen.

\subsubsection{Die Vergleiche}

Die Vergleiche der Ergebnisse der Untersuchungen in Holland und Deutschland sind wegen der Anzahl der untersuchten Kinder mit großer Vorsicht zu interpretieren. Die holländische Untersuchung umfasste 823 Kinder, die deutsche Untersuchung 330 Kinder. Wir in der Schweiz hingegen haben 70 Kinder interviewt. Diese schweizerische Untersuchung vermag so natürlich statistischen Ansprüchen nicht zu genügen und zeigt darum nur gewisse Trends auf; es lohnt sich aber trotzdem, die Resultate einander gegenüberzustellen. Ein weiterer Grund, die Vergleiche nicht überzubewerten, liegt in der oben aufgezeigten unterschiedlichen Konzeption des Kindergartens und der ersten Schuljahre. Ich werde im Folgenden einige schweizerische Daten mit verfügbaren Daten aus Holland und Deutschland vergleichen.

Eine Gegenüberstellung der holländischen Daten mit den Schweizer Daten zeigt keine großen Leistungsdifferenzen.

\begin{tabular}{|l|l|l|l||l|l|l|}
\hline & M-NL & SD-NL & N-NL & M-CH & SD-CH & N-CH \\
\hline 1. KGJ & 41.52 & 12.97 & 230 & 38.79 & 16.08 & 33 \\
\hline 2. KGJ & 57.52 & 12.31 & 315 & 60.95 & 19.92 & 37 \\
\hline Alle & 50.77 & 12.59 & 545 & 50.50 & 18.11 & 70 \\
\hline
\end{tabular}

Tab. 14 Vergleichbare Mittelwerte der holländischen und Schweizer Kinder.

Im ersten Kindergartenjahr liegen die Holländer mit etwa 2.7 Durchschnittspunkten höher als die Schweizer, hingegen im zweiten Kindergartenjahr sind die Schweizer mit etwa 3.4 Durchschnittspunkten höher als die Holländer. Es ist zu beachten, dass bei diesem Vergleich die restlichen 278 holländischen Kinder, die sich bereits im ersten Schuljahr befinden, nicht berücksichtigt wurden. Nur bei dieser Nichtberücksichtigung ist ein seriöser Vergleich möglich, da keine Schweizer Kinder den Kindergarten aus schuli- 
schen Gründen verlassen haben. Es ist bemerkenswert, dass der Durchschnitt aller holländischen Kinder vor dem ersten Schuljahr (entspricht der Stufe 3) mit 50.77 fast identisch ist mit dem Durchschnitt aller schweizerischen Kinder mit 50.50. Wir haben in unseren Untersuchungen bei den Schweizer Kindern einen markanten Leistungszuwachs zwischen der ersten Interviewphase 1998/99 und der zweiten Interviewphase 2001 festgestellt. Ein Grund dafür könnten die Bemühungen der Kindergärtnerinnen um die Umsetzung des Lehrplans sein, zum andern aber der Umstand, dass sich interessierte und anerkannt gute und fähige Kindergärtnerinnen uns ihre Kinder für die Interviews zur Verfügung gestellt haben.

Als zweiten Vergleich stelle ich in der Tab. 15 die Ergebnisse der einzelnen Komponenten der deutschen Untersuchung der schweizerischen Untersuchung gegenüber. Die

Abb. 58 stellt dann den Sachverhalt graphisch dar:

\begin{tabular}{|l|l|l|}
\hline Aspekte & $\mathrm{DE}$ & $\mathrm{CH}$ \\
\hline \hline Vergleichsbegriffe & 4.5 & 4.2 \\
\hline Klassifizierung & 3.5 & 3.5 \\
\hline Eins-Zu-Eins-Zuordnung & 3.3 & 2.8 \\
\hline Reihenfolgen & 2.1 & 1.9 \\
\hline Zahlwörter & 2.6 & 1.9 \\
\hline Strukturiertes Zählen & 2.9 & 2.0 \\
\hline Ergebnisbez. Zählen & 2.2 & 1.6 \\
\hline Allg. Zahlenwissen & 2.8 & 2.3 \\
\hline
\end{tabular}

Tab. 15 Die einzelnen Komponenten im Vergleich Deutschland - Schweiz. 


\section{Komponenten im Vergleich DE - CH}

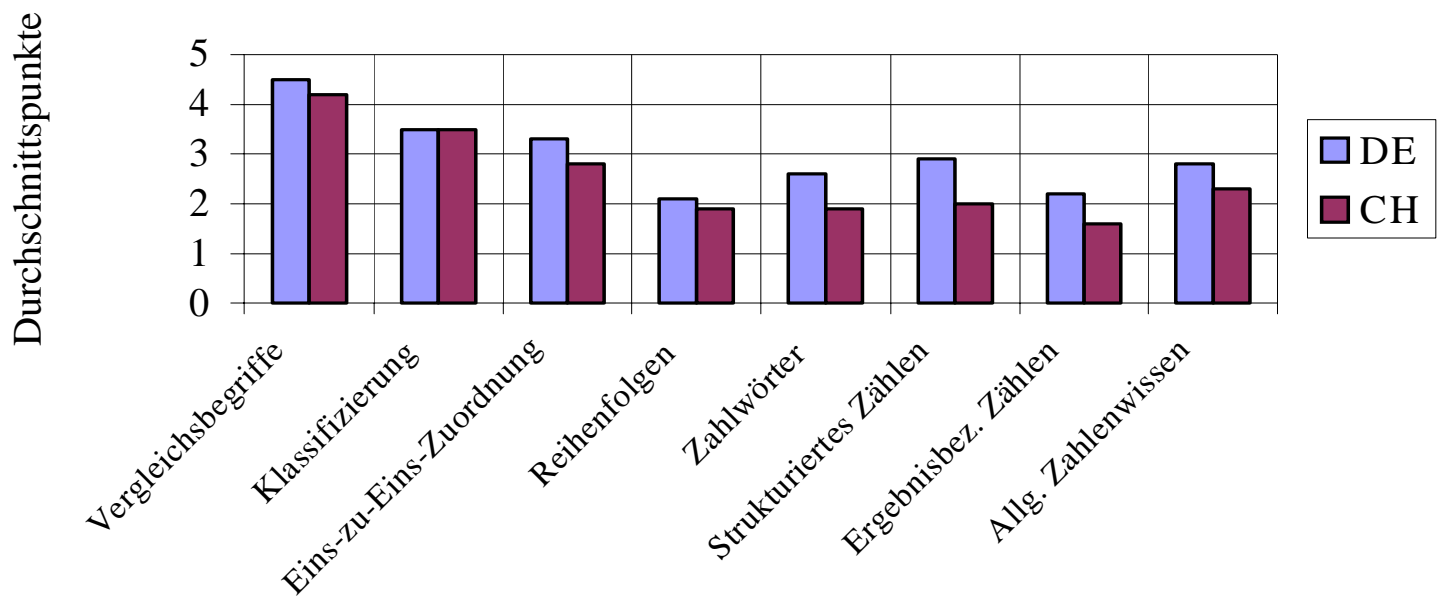

Abb. 58 Die Differenzen in den einzelnen Komponenten im Vergleich Deutschland Schweiz

In allen Komponenten sind die untersuchten Kinder aus der Schweiz den untersuchten Kindern aus Deutschland unterlegen. Vor allem bei den Komponenten, bei denen das Zählen eine Rolle spielt, sind die Leistungsunterschiede sehr ausgeprägt. Es ist aber zu bemerken, dass die deutschen Kinder beim Zeitpunkt der Untersuchung im Mittel 3.5 Monate älter als die Schweizer Kinder waren. Dass dieser Alterunterschied durchaus einen Einfluss auf das Gesamtergebnis hat, zeigte Hasemann bei seinen Untersuchungen, die er in Deutschland im Februar/März und Juni 1997 durchführte. Er hat festgestellt, dass ein Zuwachs von etwa 21/2 Punkten innert gut drei Monaten stattfindet (Hasemann 1998, 265). Das sind nach der Normierung 6.25 Punkte und damit relativiert sich der Kompetenzvorsprung der deutschen Kinder beträchtlich.

Zum Schluss geben die Tab. 16 und Abb. 59 noch die Verteilung der erreichten Punkte der deutschen Kinder wieder.

\begin{tabular}{|c|c|}
\hline Punkte & Anzahl \\
\hline \hline $0-5$ & 1 \\
\hline $6-10$ & 14 \\
\hline $11-15$ & 25 \\
\hline $16-20$ & 68 \\
\hline $21-25$ & 84 \\
\hline $26-30$ & 76 \\
\hline
\end{tabular}




\begin{tabular}{|l|l|}
\hline $31-35$ & 51 \\
\hline $36-40$ & 11 \\
\hline
\end{tabular}

Tab. 16 Die Punkteverteilung in Deutschland. Maximum sind 40 Punkte. $N=330$.

\section{Verteilung Deutschland}

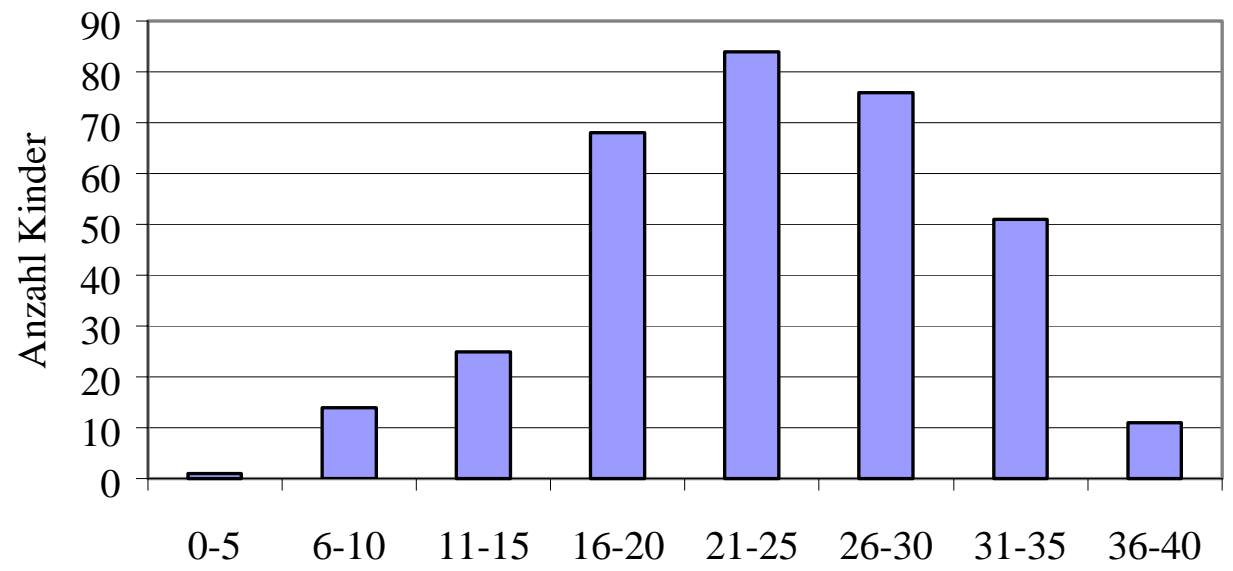

Abb. 59 Eine schöne Verteilung nach rechts verschoben (siehe Hasemann 1998).

Zum Vergleich dasselbe für die Schweizer Kinder in Tab. 17 und Abb. 60:

\begin{tabular}{|c|c|}
\hline Punkte & Anzahl \\
\hline \hline $0-5$ & 2 \\
\hline $6-10$ & 7 \\
\hline $11-15$ & 13 \\
\hline $16-20$ & 16 \\
\hline $21-25$ & 14 \\
\hline $26-30$ & 11 \\
\hline $31-35$ & 6 \\
\hline $36-40$ & 1 \\
\hline
\end{tabular}

Tab. 17 Die Punkteverteilung in der Schweiz. Maximum sind 40 Punkte. $N=70$. 


\section{Verteilung Schweiz}

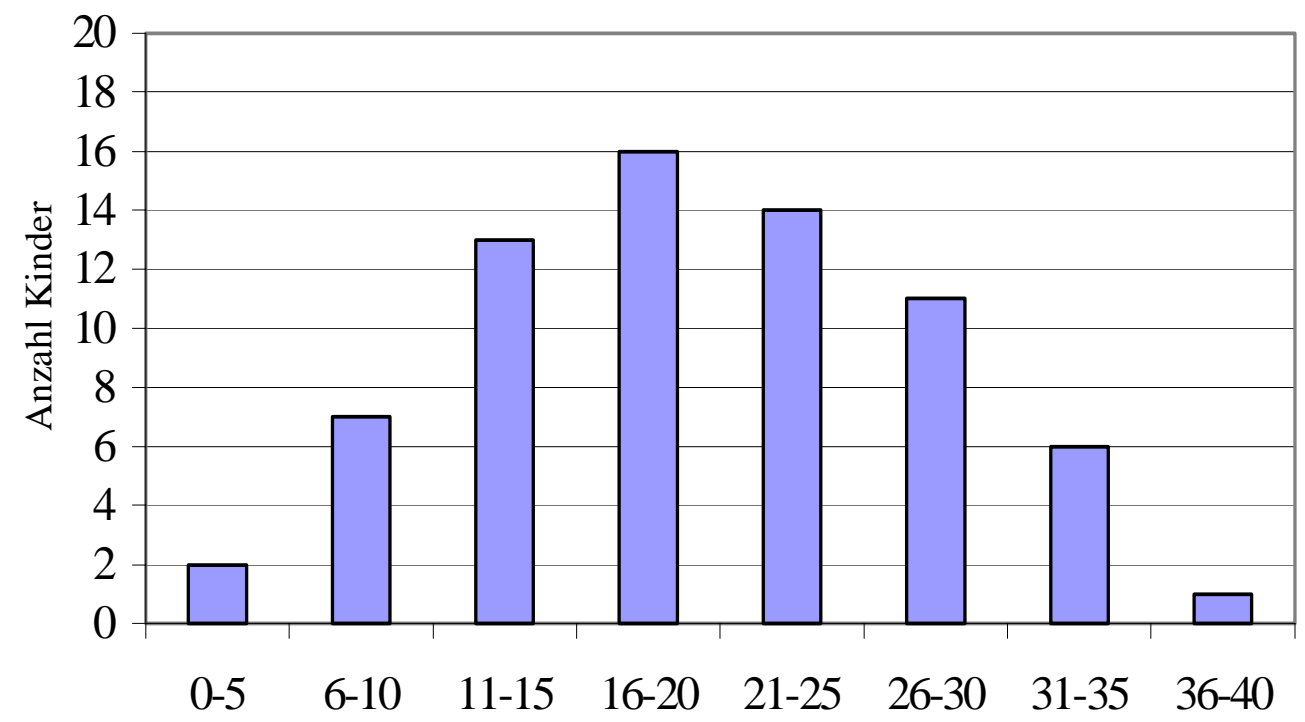

Abb. 60 Auch hier eine schöne Verteilung.

Die Gegenüberstellung der zwei Verteilungen aus Deutschland und der Schweiz zeigt, dass der Test in Deutschland eher zu leicht und in der Schweiz eher zu schwer ist. 


\subsection{Die Piaget'schen Komponenten in Wechselwirkung zur Zählkompe- tenz}

$\mathrm{Zu}$ Beginn einer Feinanalyse der numerischen Kompetenz möchte ich die Wechselwirkung der vier - auf Arbeiten Piagets basierenden - Komponenten Vergleichskonzepte, Klassifizierungen, Zuordnungen und Reihenfolgen einerseits und die Zählkompetenz andererseits untersuchen. Dabei werden die Ergebnisse dieser vier genannten Komponenten in Bezug gebracht zu erfolgreichem Zählvermögen und umgekehrt. Die Grundfragen lauten: Ist besseres Abschneiden in den vier Komponenten Garant dafür, dass die Kinder über ein besseres Zählvermögen verfügen und ist hohe Zählkompetenz umgekehrt auch Garantie dafür, dass diese Kinder die vier genannten Komponenten besser bewältigen? Besteht also ein Zusammenhang zwischen der Bewältigung der Piaget'schen Komponenten und einem guten Zählvermögen? Für diese Analyse definiere ich als Zählkompetenz die Bewältigung der Aufgaben A21 (,Zähle bis zwanzig“) und/oder A28 ([Ex. legt 20 Holzwürfel in einem Haufen mit etwas Abstand zwischen den Holzwürfeln auf den Tisch.] „Zähle diese Holzwürfel.“). Ein Kind, das eine oder beide dieser Aufgaben löst, wird den „Zählern“ zugeordnet, die übrigen den „Nichtzählern“. Diese zwei Aufgaben bieten eine gute Möglichkeit, die Zählkompetenz kurz und prägnant aufzuzeigen und zwar einerseits mit dem Aufsagen der Zahlwortreihe ohne konkreten Bezug zu irgendwelchen Objekten und andererseits mit dem konkreten Abzählen einer Kollektion von 20 Gegenständen. Die Aufgabe A21 wurde von 53\% (siehe Seite 120), die Aufgabe A28 von 43\% (siehe Seite 124) der Kinder gelöst. In konkreten Zahlen ausgedrückt heißt das, dass insgesamt 43 von 70 Kindern die Aufgaben A21 und/oder A28 gelöst haben (25 Kinder haben beide Aufgaben gelöst, 13 Kinder nur die Aufgabe A21 und 5 Kinder nur die Aufgabe A28). In der vorliegenden Schweizer Untersuchung bekam die Aufgabe A21 einen Schwierigkeitsindex ${ }^{34}$ von 54.3 (mittelschwere Aufgabe) und die Aufgabe A28 einen solchen von 42.9; die Aufgabe A28 ist also eine etwas schwerere Aufgabe. Diese Schwierigkeitsindizes korrespondieren sehr gut mit der auf den holländischen Untersuchungsdaten basierenden Schwierigkeitsskala (siehe Seite 87), an die ich mich bei der Einschätzung der Hierarchien bezüglich Schwierigkeit der einzelnen Aufgaben orientieren werde.

\footnotetext{
${ }^{34}$ Der Schwierigkeitsindex ist gleich dem prozentualen Anteil $P$ der richtigen Antworten bei $N$ Fragen. Der Schwierigkeitsindex ist bei leichten Fragen hoch, bei schwierigen Fragen tief (vgl. Lienert/Raatz 1998, 73).
} 


\subsubsection{Die Vergleichskonzepte in Wechselwirkung mit Zählkompetenz}

\subsubsection{Resultate}

Die fünf Aufgaben der Komponente Vergleichskonzepte ${ }^{35}$ haben die Kinder mit einem sehr hohen Durchschnitt von $4.2^{36}$ gelöst. Um den Zusammenhang zum Zählen einigermaßen fundiert zu gestalten, betrachte ich nur diejenigen Kinder, die alle fünf Aufgaben gelöst haben und bringe diese wie oben geschildert in Bezug zu der Bewältigung der Aufgabe A21 und A28. 37 Kinder haben alle fünf Aufgaben der Komponente Vergleichskonzepte gelöst, davon 26 auch die Aufgaben A21 und/oder A28. Die restlichen 11 Kinder haben weder Aufgabe A21 noch Aufgabe A28 gelöst.

Für eine formale Ausgestaltung der Beziehung zwischen den zu untersuchenden vier Komponenten und dem Zählen, insbesondere die Bestimmung der Trennschärfe ${ }^{37}$, eignet sich der sog. Punkt-Vierfelder-Korrelationskoeffizient, kurz Vierfelder- oder PhiKoeffizient genannt (Lienert/Raatz 1998, 91), wobei ich als Außenkriterium (ebd. 84) bei den Vergleichskonzepten natürlich „Erfolg/Misserfolg“ benutze, d.h. „Erfolg“ bei der Lösung aller fünf Vergleichskonzeptaufgaben, sonst „Misserfolg“. Bei der Bestimmung der Trennschärfe werden die Probanden in zwei Gruppen zusammengefasst; in eine obere Gruppe (die Erfolgsgruppe) und in eine untere Gruppe (die Misserfolgsgruppe). Bei den in diesem Abschnitt zu untersuchenden Vergleichkonzepten sind 37 Kinder in der oberen Gruppe (haben alle fünf Aufgaben gelöst) und die restlichen 33 Kinder in der unteren Gruppe. In der oberen Gruppe hat es 26 „Zähler“(+) und 11 „Nichtzähler“ (-), in der unteren Gruppe 17 ,Zähler“ und 16 „Nichtzähler“. Diese Angaben generieren die Vierfelder-Tafel wie folgt:

\begin{tabular}{|l|c|c|c|}
\hline & Zähler (+) & Nichtzähler (-) & $\sum$ \\
\hline Obere Gruppe: Erfolg & $a=26$ & $b=11$ & 37 \\
\hline Untere Gruppe: Misserfolg & $c=17$ & $d=16$ & 33 \\
\hline$\sum$ & 43 & 27 & 70 \\
\hline
\end{tabular}

Tab. 18 Die Vierfeldertafel

\footnotetext{
${ }^{35}$ Die einzelnen Aufgaben sind ab Seite 104 vorgestellt.

${ }^{36}$ Von 5 möglichen Durchschnittspunkten

${ }^{37}$ Die Trennschärfe bringt zum Ausdruck, dass die Aufgabe (Aufgabengruppe) die Kinder mit hohem Gesamtpunktwert von den Kindern mit niedrigem Punktwert mehr oder weniger scharf trennt (Lienert/Raatz 1998, 30).
} 
Die Trennschärfe der Aufgabengruppe Vergleichskonzepte - Zählkompetenz berechnet sich nach der Formel

$$
r=\frac{a d-b c}{\sqrt{(a+b)(c+d)(a+c)(b+d)}}(\text { siehe Lienert/Raatz 1998, 91) }
$$

Formel 1

In unserem Fall ergibt sich als Koeffizient $r=0.19$, was eine geringe Trennschärfe bedeutet $^{38}$. Das heißt nun, dass das Zählen fast genauso gut von den in den Vergleichskonzepten erfolgreichen wie von den in den Vergleichskonzepten weniger erfolgreichen Kindern bewältigt wird.

Die erfolgreichen Zähler haben umgekehrt die Vergleichskonzeptaufgaben mit einem Durchschnitt von 4.4 Punkten gelöst, die Nichtzähler mit ebenfalls noch hohen 3.9 Durchschnittspunkten. Zur Kontrolle lässt sich auch hier die Trennschärfe berechnen, wobei als Außenkriterium „Zähler/Nichtzähler“ genommen wird und „Erfolg“ dann die Bewältigung der fünf Vergleichskonzeptaufgaben bedeutet. Die Berechnung auf dieser Grundlage muss auch hier die gleiche Trennschärfe von $r=0.19$ ergeben (siehe dazu Seite 267 im Anhang).

\subsubsection{Folgerung}

Obwohl von den 37 erfolgreichen Vergleichskonzeptaufgabenlöser 26 auch erfolgreiche Zähler sind, lassen diese Zahlen nicht den Schluss zu, dass für eine hohe Zählkompetenz auch das Verstehen von Vergleichkonzepten Voraussetzung ist, denn die 11 in dieser Sparte erfolgreichen Kinder verfügen über wenig oder keinerlei Zählkompetenz. Diese Aussage bestätigt auch die Trennschärfe von $r=0.19$.

Auf der anderen Seite sind die Zähler mit 4.4 Durchschnittspunkten gegenüber 3.9 Durchschnittspunkten der Nichtzähler zwar ein wenig erfolgreicher bei der Bewältigung der Vergleichkonzeptaufgaben, diese Differenz ist aber sehr bescheiden und weist nicht

\footnotetext{
${ }^{38}$ Ein hoher Trennschärfekoeffizient (das Maximum ist 1) besagt, dass die entsprechende Aufgabe (Aufgabengruppe) leistungsfähige Probanden von weniger leistungsfähigen Probanden deutlich unterscheidet, indem leistungsfähige Probanden die Aufgabe meist richtig, weniger leistungsfähige Probanden die Aufgabe meist falsch lösen. Ein Trennschärfekoeffizient um 0 bringt zum Ausdruck, dass die Aufgabe von „guten“ und „schlechten“ Probanden etwa gleich häufig beantwortet resp. gelöst wird. Ein negativer Trennschärfekoeffizient besagt, dass die Aufgabe von den ,guten“ Probanden schlechter gelöst wird als von den ,schlechten“ Probanden (vgl. Lienert/Raatz 1998, 78).
} 
auf eine große Beeinflussung der Zählkompetenz auf Vergleichsaufgaben hin. Als Ergebnis lässt sich nun sagen, dass eine gute Kenntnis der Vergleichsbegriffe höher, niedriger, dicker, dünner, breiter, schmaler, mehr, weniger, wenigsten usw. keine hinreichende Bedingung für erfolgreiches Zählen darstellt und umgekehrt Zählkompetenz nur geringe Auswirkung auf Vergleichkonzepte ausübt. Die 17 Zähler der Misserfolgsgruppe beweisen, dass das Verstehen von Vergleichskonzepten keine Voraussetzung für Zählkompetenz ist - die 11 Nichtzähler der Erfolgsgruppe beweisen, dass Zählen keine Voraussetzung fürs Vergleichen ist.

\subsubsection{Die Klassifizierung in Wechselwirkung mit Zählkompetenz}

\subsubsection{Resultate}

Die fünf Aufgaben der Komponente Klassifizierung ${ }^{39}$ haben die Kinder mit einem Durchschnitt von 3.5 gelöst. Da es bei diesen Aufgaben schon mal vorkommen kann, dass die Kinder eine Aufgabe nicht genau verstehen oder anders als der Interviewer interpretieren, nehme ich als Kriterium für eine erfolgreiche Bewältigung dieser Komponente vier gelöste Aufgaben; 39 Kinder erfüllten dieses Kriterium. Darunter befinden sich 30 Zähler und 9 Nichtzähler. Man beachte, dass von den übrigen 31 Kindern, die drei oder weniger Punkte bei den Klassifizierungsaufgaben erreichten, nur gerade 13 den Zählern zugeordnet werden können. Die Vierfelder-Tafel sieht in diesem Fall wie folgt aus:

\begin{tabular}{|l|c|c|c|}
\hline & Zähler (+) & Nichtzähler (-) & $\sum$ \\
\hline Obere Gruppe: Erfolg & 30 & 9 & 39 \\
\hline Untere Gruppe: Misserfolg & 13 & 18 & 31 \\
\hline$\Sigma$ & 43 & 27 & 70 \\
\hline
\end{tabular}

Tab. 19 Vierfeldertafel Klassifizierung - Zählkompetenz

Die Trennschärfe $r=0.35$ der Aufgabengruppe Klassifizierung - Zählkompetenz zeigt einen weit höheren statistischen Zusammenhang als bei der Aufgabengruppe Ver-

${ }^{39}$ Die einzelnen Aufgaben sind ab Seite 108 vorgestellt. 
gleichskonzepte - Zählkompetenz, was besagt, dass die guten „Klassifizierer“ die Zählaufgaben besser lösen als die weniger guten „Klassifizierer“.

Auf der andern Seite erreichen die 43 Zähler eine hohe Durchschnittspunktzahl von 4.0 bei den fünf Klassifizierungsaufgaben, wo hingegen die Nichtzähler nur auf 2.9 Durchschnittspunkten kommen.

\subsubsection{2. $\quad$ Folgerung}

Auch in diesem Fall stellt sich die Sachlage ähnlich dar wie bei der vorigen Komponente: Obwohl von den 39 erfolgreichen Klassifizierungsaufgabenlöser 30 auch erfolgreiche Zähler sind, lassen auch diese Zahlen nicht den Schluss zu, dass für eine hohe Zählkompetenz auch das Verstehen von Klassifizierungskonzepten Voraussetzung ist, denn 13 Zähler in der unteren Gruppe verfügen über kleine Klassifizierungskompetenzen.

Ähnlich präsentiert sich die Lage umgekehrt, wenn man von den Zählern ausgeht. Diese sind deutlich erfolgreicher bei der Bewältigung der Klassifizierungsaufgaben als die Nichtzähler; der doch relativ hohe Korrelationskoeffizient von 0.35 lässt vermuten, dass hier immerhin eine kleine Wechselwirkung Klassifizierung $\leftrightarrow$ Zählkompetenz auszumachen ist. Wie aber die doch große Anzahl von 13 Kindern zeigt, die zählen können, ohne Klassifizierungskompetenzen zu besitzen, ist es für eine hohe Zählkompetenz aber in keiner Weise zwingend nötig, dass die Kinder Klassifizierungsmerkmale wie „alle außer“, „,mit ..., aber ohne ... “, „, alle mit ..., aber ohne ..., und mit ... “ beherrschen.

\subsubsection{Die Zuordnungskomponente in Wechselwirkung mit Zählkompetenz}

\subsubsection{Resultate}

Die fünf Aufgaben der Komponente Zuordnungen ${ }^{40}$ haben die Kinder mit einem Durchschnitt von 2.8 gelöst. Da es auch bei diesen Aufgaben vorkommen kann, dass die Kinder eine Aufgabe nicht genau verstehen oder anders als der Interviewer interpretieren und zudem die Aufgabe A15 (siehe Seite 114) nur gerade von 24\% der Kinder gelöst wurde, nehme ich als Kriterium für eine erfolgreiche Bewältigung dieser Komponente wiederum vier gelöste Aufgaben; unter dieser Voraussetzung waren 21 Kinder erfolg-

${ }^{40}$ Die einzelnen Aufgaben sind ab Seite 111 vorgestellt. 
reich. ${ }^{41}$ Von den 21 in der Komponente Zuordnung erfolgreichen Kindern sind 20 Zähler, also fast alle. Dieser überragende Anteil an Zähler ist ein Indiz dafür, dass die Einszu-Eins-Zuordnung und die Zählkompetenz einer gegenseitigen Beeinflussung unterworfen sind. Dies wird ebenfalls sehr schön dokumentiert bei der Analyse der Aufgabe A12 (siehe Seite 112), bei der die Kinder die zwei Würfelmuster 5 und 6 aus einem Vorrat von 15 Holzwürfel nachlegen sollten: von den 46 bei dieser Aufgabe erfolgreichen Kindern befindet sich von den gesamthaft 43 Zählern der übergroße Anteil von 35 Kindern. Die Vierfeldertafel hat nun folgendes Bild:

\begin{tabular}{|l|c|c|c|}
\hline & Zähler (+) & Nichtzähler (-) & $\sum$ \\
\hline Obere Gruppe: Erfolg & 20 & 1 & 21 \\
\hline Untere Gruppe: Misserfolg & 23 & 26 & 49 \\
\hline$\sum$ & 43 & 27 & 70 \\
\hline
\end{tabular}

Tab. 20 Vierfeldertafel Zuordnungskomponente - Zählkompetenz.

Daraus berechnet sich der Korrelationskoeffizient $r=0.45$.

Alternativ zu vier gelösten Aufgaben für Erfolg habe ich im Folgenden nur drei gelöste Aufgaben für Erfolg berücksichtigt. Die beiden Gruppengrößen rücken dadurch etwas näher zusammen. Der Trennschärfekoeffizient reduziert sich dabei auf $r=0.40$.

\begin{tabular}{|l|c|c|c|}
\hline & Zähler (+) & Nichtzähler (-) & $\sum$ \\
\hline Obere Gruppe: Erfolg & 33 & 10 & 43 \\
\hline Untere Gruppe: Misserfolg & 10 & 17 & 27 \\
\hline$\sum$ & 43 & 27 & 70 \\
\hline
\end{tabular}

Tab. 21 Vierfeldertafel Zuordnungskomponente - Zählkompetenz (alternativ)

Umgekehrt erreichten die 43 Zähler bei den fünf Zuordnungssaufgaben, eine Durchschnittspunktzahl von 3.2, wo hingegen die Nichtzähler nur auf 2.2 Durchschnittspunkte kamen.

\footnotetext{
${ }^{41}$ Von 17 Kindern, die die Aufgabe A15 gelöst haben, befinden sich 11 in der Gruppe der erfolgreichen Zuordnungskomponentenlöser.
} 


\subsubsection{Folgerung}

Anders als bei den beiden vorangegangenen Komponenten Vergleichskonzepte und Klassifizierung ist bei der Zuordnungskomponente die Wechselwirkung doch um einiges höher und auffälliger. Der hohe Trennschärfekoeffizient mit $r=0.45$ ( $r=0.40$ bei drei gelösten Zuordnungsaufgaben) dokumentiert diesen Zusammenhang deutlich. Auffällig ist die Tatsache, dass von den 21 erfolgreichen Kindern bei den Zuordnungsaufgaben 20 ebenfalls erfolgreiche Zähler sind.

8.2.4 Die Reihenfolgekomponente in Wechselwirkung mit Zählkompetenz

\subsubsection{Resultate}

Bei der Komponente Reihenfolgen ${ }^{42}$ haben die Kinder eine tiefe Durchschnittspunktzahl von 1.9 erreicht. Ein Grund dafür ist sicher die für Schweizer Kinder sehr schwer verständliche Toastaufgabe $\mathrm{A} 20^{43}$, die von nur $17 \%$ der Kinder korrekt gelöst wurde (siehe Seite 118). Aus diesem Grund betrachte ich Kinder, die drei und mehr Punkte in dieser Komponente erreicht haben, als erfolgreich. 26 Kinder sind auf Grund dieses Kriteriums erfolgreich; darunter befinden sich 24 Zähler und nur 2 Nichtzähler. Auch hier überrascht dieser überragende Anteil an Zählern nicht; denn auch hier scheint es, dass die Zählkompetenz und die Reihenfolgebildung in einer intensiven Wechselwirkung zueinander stehen. Die Vierfeldertafel hat folgendes Bild:

\begin{tabular}{|l|c|c|c|}
\hline & Zähler (+) & Nichtzähler (-) & $\sum$ \\
\hline Obere Gruppe: Erfolg & 24 & 2 & 26 \\
\hline Untere Gruppe: Misserfolg & 19 & 25 & 44 \\
\hline$\sum$ & 43 & 27 & 70 \\
\hline
\end{tabular}

Tab. 22 Vierfeldertafel Reihenfolgen - Zählkompetenz.

\footnotetext{
${ }^{42}$ Die einzelnen Aufgaben sind ab Seite 115 vorgestellt.

${ }^{43}$ Schwierigkeitsindex 24.3
} 
Daraus berechnet sich der Korrelationskoeffizient: $r=0.49$.

Auch bei dieser Komponente reduziere ich versuchshalber die Anzahl der zu lösenden Aufgaben um eins, um so ausgeglichene Gruppengrößen zu erhalten. Mit jetzt zwei gelösten Aufgaben als Erfolg werden die Gruppengrößen mit je 35 Kindern ideal. Die zugehörige Vierfeldertafel mit dem sich daraus ergebenden Trennschärfekoeffizienten $r=$ 0.38 ist unten abgebildet:

\begin{tabular}{|l|c|c|c|}
\hline & Zähler (+) & Nichtzähler (-) & $\sum$ \\
\hline Obere Gruppe: Erfolg & 28 & 7 & 35 \\
\hline Untere Gruppe: Misserfolg & 15 & 20 & 35 \\
\hline$\Sigma$ & 43 & 27 & 70 \\
\hline
\end{tabular}

Tab. 23 Vierfeldertafel Reihenfolgen - Zählkompetenz (alternativ)

Umgekehrt erreichten die 43 Zähler bei den fünf Reihenfolgenaufgaben eine Durchschnittspunktzahl von 2.4, wo hingegen die Nichtzähler nur auf 1.2 Durchschnittspunkte kamen.

\subsubsection{Folgerung}

Ganz ähnlich wie bei der Komponente Eins-zu-eins-Zuordnungen ist auch bei der Komponente Reihenfolgen die Wechselwirkung zur Zählkompetenz auffällig. Der statistische Zusammenhang wird in diesem Vergleich mit dem höchsten Trennschärfekoeffizient von $r=0.49$ ( $r=0.38$ bei zwei gelösten Aufgaben als Erfolg) sehr schön dokumentiert. Die Bewältigung von Reihenfolgen ist für Zählkompetenz nach der Analyse der Daten zwar nicht zwingend, aber doch immerhin förderlich.

\subsubsection{Zusammenfassung der Analyse}

Die unten aufgeführte Tab. 24 zeigt als Zusammenstellung die Trennschärfekoeffizienten der vier Piaget'schen Komponenten im Vergleich zur Zählkompetenz. Daraus wird 
ersichtlich, dass die Bedeutung der Piaget'schen Komponenten bezüglich der Zählkompetenz in der Gesamtheit doch als recht bescheiden bezeichnet werden kann. Das gute Beherrschen der Eins-zu-eins-Zuordnungen und der Reihenfolgen sind zwar nicht Voraussetzung für eine Zählkompetenz, aber, wie die Trennschärfekoeffizienten $r=0.45$ und $r=0.49$ zeigen, besteht doch eine Wechselwirkung zwischen diesen beiden Komponenten, die durchaus förderlich für eine hohe Zählkompetenz sein kann. Diese gegenseitige Beeinflussung ist aber doch relativ gemäßigt und weit geringer, als gemeinhin erwartet.

Das Ergebnis ist auf den ersten Blick überraschend, war man doch bisher der Überzeugung, dass eben die Beherrschung dieser Komponenten zwingend ist für den Erwerb von Zählkompetenz. Alle vier Komponenten sind aber in keiner Weise notwendig für die Erlangung von Zählkompetenz. Viele Kinder zählen auch dann erfolgreich, wenn das Lösen von Aufgaben in den vier Komponenten sehr unsicher und instabil ist.

\begin{tabular}{|l|l|l|l|l|}
\hline & Vergleichen & Klassifizierungen & Zuordnungen & Reihenfolgen \\
\hline $\begin{array}{l}\text { Komponenten } \leftrightarrow \\
\text { Zählen }\end{array}$ & $r=0.19$ & $r=0.35$ & $\begin{array}{l}r=0.45 \\
(r=0.40)\end{array}$ & $\begin{array}{l}r=0.49 \\
(r=0.38)\end{array}$ \\
\hline
\end{tabular}

Tab. 24 Trennschärfekoeffizienten der vier Piaget'schen Komponenten und Zählkompetenz.

Die Konsequenz dieser Erkenntnis ist, dass irgendetwas außerhalb der erwähnten Piaget'schen Komponenten für die Erlangung der Zählkompetenz mitverantwortlich gemacht werden muss. Es drängt sich die allgemeine Leistungsfähigkeit des Kindes, der Intelligenzquotient oder das Alter auf. Da es für die Schweizer Untersuchung nicht möglich war, auch eine Intelligenzerhebung der Kinder durchzuführen, bleibt für einen Vergleich nur die allgemeine Leistungsfähigkeit und das Alter der Kinder.

\subsubsection{Die Abhängigkeit der Erfolge in den vier Komponenten vom Alter}

Für diese Analyse werden die Kinder in zwei Gruppen unterteilt: in die erste Kindergartenjahrgruppe (1. KgJ) und in die zweite Kindergartenjahrgruppe (2. KgJ). In der ersten Gruppe befinden sich 33 Kinder, in der zweiten 37; diese Gruppengrößen eignen sich 
gut für eine Trennschärfeanalyse mit der Vierfeldertafel, wobei als „Erfolg“ die Zugehörigkeit in die 2. KgJ-Gruppe bedeutet und „Misserfolg“ in die Zugehörigkeit der 1. KgJGruppe $^{44}$.

Alter $\rightarrow$ Vergleichkonzepte

\begin{tabular}{|l|l|l|l|}
\hline & + & - & $\sum$ \\
\hline $2 . \mathrm{KgJ}$ & 21 & 16 & 37 \\
\hline $1 . \mathrm{KgJ}$ & 16 & 17 & 33 \\
\hline$\sum$ & 37 & 33 & 70 \\
\hline
\end{tabular}

Tab. 25 Vierfeldertafel Alter $\rightarrow$ Vergleichskonzepte

Die Trennschärfe beträgt $r=0.08$, d.h. das Alter spielt für die Lösungskompetenz der Vergleichskonzeptaufgaben überhaupt keine Rolle.

Für die Analyse der übrigen drei Komponenten verzichte ich auf die Darstellung der Tabellen, die im Anhang ab Seite 268 aufgeführt sind. In der folgenden Tab. 26 sind die daraus berechneten Korrelationskoeffizienten aufgeführt:

\begin{tabular}{|l|l|l|l|l|}
\hline & Vergleichen & Klassifizierungen & Zuordnungen & Reihenfolgen \\
\hline 2. KgJ/1. KgJ & $r=0.08$ & $r=0.14$ & $r=0.43$ & $r=0.43$ \\
\hline
\end{tabular}

Tab. 26 Trennschärfekoeffizienten Alter und der vier Piaget'schen Komponenten

Es erstaunt doch sehr, dass das Alter auf das erfolgreiche Lösen der vier Piaget'schen Komponenten einen sehr bescheidenen Einfluss ausübt, durchaus vergleichbar mit den in den vorigen Kapiteln betrachteten Beziehungen Zählen $\leftrightarrow$ Piaget’schen Komponenten. Wie sieht es nun mit der allgemeinen Leistungsfähigkeit aus?

8.2.5.2 Die Abhängigkeit der Erfolge von der allgemeinen Leistungsfähigkeit

\footnotetext{
${ }^{44}$ Zur Vermeidung von negativen Trennschärfekoeffizienten wird diese Erfolgszuteilung gewählt.
} 
Die allgemeine Leistungsfähigkeit wird in dieser Arbeit über das Gesamtergebnis des Tests definiert. Erreicht ein Proband 50 (von 100) und mehr Punkte, gehört er in die Gruppe der Erfolgreichen (Erfolgreich), sonst in die andere Gruppe (Nicht erfolgreich). Unter diesen Umständen sind die zwei Gruppengrößen mit je 35 Kindern identisch.

\section{Leistungsfähigkeit $\rightarrow$ Vergleichkonzepte}

\begin{tabular}{|l|c|c|c|}
\hline & + & - & $\Sigma$ \\
\hline Erfolgreich & 26 & 9 & 35 \\
\hline Nicht erfolgreich & 11 & 24 & 35 \\
\hline$\sum$ & 37 & 33 & 70 \\
\hline
\end{tabular}

Tab. 27 Vierfeldertafel Leistungsfähigkeit $\rightarrow$ Vergleichskonzepte

Hier beträgt der Trennschärfekoeffizient für diese Kombination einen mittleren Wert von $r=0.43$. Aber die übrigen Trennschärfekoeffizienten lassen aufhorchen (Tabellen siehe im Anhang ab Seite 268). Die daraus berechneten Trennschärfekoeffizienten sind in der untenstehenden Tab. 28 aufgelistet.

\begin{tabular}{|l|l|l|l|l|}
\hline & Vergleichen & Klassifizierungen & Zuordnungen & Reihenfolgen \\
\hline Erfolgreich/Nicht erfgr. & $r=0.43$ & $r=0.55$ & $r=0.53$ & $r=0.65$ \\
\hline
\end{tabular}

Tab. 28 Trennschärfekoeffizienten Leistungsfähigkeit und der vier Piaget'schen Komponenten

Im Vergleich mit allen anderen Beziehungen ist der statistische Zusammenhang bei diesen Kombinationen am augenfälligsten und die Vermutung drängt sich auf, dass die allgemeine Leistungsfähigkeit für Erfolg auf allen Gebieten das ausschlaggebende Moment darstellt. Um diese These zu stützen, untersuche ich im Folgenden die Beziehung Alter $\rightarrow$ Zählkompetenz und in einem weiteren Schritt die Beziehung Allgemeine Leistungsfähigkeit $\rightarrow$ Zählkompetenz.

Alter $\rightarrow$ Zählkompetenz 
Wie schon oben dargestellt, sind in der „Erfolgsgruppe“ die Kinder aus dem 2. Kindergartenjahr. Die Vierfeldertafel hat folgendes Aussehen und gibt über den Trennschärfekoeffizienten Antwort auf die Frage, ob denn die älteren Kinder die Zählaufgaben besser lösen als die jüngeren Kinder. Losgelöst von allen anderen Beziehungen scheint eine solche Frage eigentlich überflüssig, da die Antwort schon gegeben ist. Erst im Zusammenhang mit der nächsten Fragestellung rückt eine solche Frage aber in ein anderes Licht:

\begin{tabular}{|l|c|c|c|}
\hline & + & - & $\sum$ \\
\hline $2 . \mathrm{KgJ}$ & 32 & 5 & 37 \\
\hline $1 . \mathrm{KgJ}$ & 11 & 22 & 33 \\
\hline$\sum$ & 43 & 27 & 70 \\
\hline
\end{tabular}

Tab. 29 Vierfeldertafel Alter $\rightarrow$ Zählkompetenz

Der Korrelationskoeffizient ist mit $r=0.55$ wie erwartet tatsächlich hoch, d.h., dass ein hoher Zusammenhang zwischen dem Alter und der Zählkompetenz herrscht.

Leistungsfähigkeit $\rightarrow$ Zählkompetenz

\begin{tabular}{|l|c|c|c|}
\hline & + & - & $\Sigma$ \\
\hline Erfolgreich & 34 & 1 & 35 \\
\hline Nicht erfolgreich & 9 & 26 & 35 \\
\hline$\sum$ & 43 & 27 & 70 \\
\hline
\end{tabular}

Tab. 30 Vierfeldertafel Leistungsfähigkeit $\rightarrow$ Zählkompetenz.

Der Trennschärfekoeffizient zeigt den höchsten in dieser Untersuchung gemessenen Wert von $r=0.73$, d.h., dass die Zählkompetenz vor allem von der allgemeinen Leistungsfähigkeit abhängt und weniger von speziellen Kompetenzen in den Piaget'schen Komponenten Vergleichen, Klassifizieren, Eins-zu-eins-Zuordnungen oder Reihenfolgen. Die weitere Analyse bestärkt die Vermutung, dass also nicht diese Piaget'schen 
Bereiche ausschlaggebend für Zählkompetenz sind, sondern schlicht die allgemeine Leistungsfähigkeit, die natürlich vielfach mit dem Alter gekoppelt ist.

Weil diese Aussage von tief greifender Bedeutung ist, möchte ich die Abhängigkeiten in der unten abgebildeten Tab. 31 nochmals zusammenfassen. Dabei sind in der ersten Spalte jeweils die Beziehungen und in der zweiten Spalte die Korrelationskoeffizienten aufgeführt:

\begin{tabular}{|l|l|}
\hline Vergleichkomponente - Zählkompetenz & $r=0.19$ \\
\hline Klassifizierung - Zählkompetenz & $r=0.35$ \\
\hline Eins-Zu-eins-Zuordnungen - Zählkompetenz & $r=0.45(r=0.40)$ \\
\hline Reihenfolgen - Zählkompetenz & $r=0.49(r=0.38)$ \\
\hline Alter - Zählkompetenz & $r=0.55$ \\
\hline Allgemeine Leistungsfähigkeit - Zählkompetenz & $r=0.73$ \\
\hline
\end{tabular}

Tab. 31 Zusammenstellung Korrelationskoeffizienten

Nicht die Zusammenhänge Vergleichskonzepte - Zählkompetenz, Klassifizierung Zählkompetenz, Eins-zu-eins-Zuordnungen - Zählkompetenz, und Reihenfolgen Zählkompetenz bestimmen in erster Linie die Zahlbegriffsentwicklung, sondern es wird sehr deutlich, dass das Alter und vor allem die allgemeine Leistungsfähigkeit alle andern Einflüsse überragt. Die Kinder sind in der Phase der Zahlbegriffsbildung bereit, sich auf allen Gebieten Kompetenzen anzueignen und die Entwicklung läuft parallel und weniger in einer zeitlichen Abfolge. Ein Kind, das in der Lage ist, die Zahlwortreihe bis 20 sicher und solide aufzusagen, ist mit hoher Wahrscheinlichkeit auch in der Lage, multiple Klassifikationsaufgaben, Eins-zu-eins-Aufgaben oder Reihenfolgeprobleme zu lösen und umgekehrt. Nicht das eine ist dem andern vorgelagert, sondern die Kompetenzentwicklung läuft gleichzeitig ab. Diese Zeit der Konsolidierung der Zählkompetenz ist zeitlich sehr individuell; unsere Untersuchung deutet auf einen Zeitrahmen vom vierten bis zum sechsten Lebensjahr hin.

Fazit: Für die Erlangung einer soliden Zählkompetenz sind in erster Linie die allgemeine Leistungsfähigkeit gekoppelt mit dem Lebensalter und erst in zweiter Linie Sekundärkompetenzen im Piaget'schen Sinne verantwortlich. Im Folgenden werden zur Unterstützung dieser These einzelne Aufgaben herausgegriffen und Beziehungen untereinander genauer untersucht. 


\subsection{Pränumerische und numerische Kompetenzen im Vergleich}

Um den Grund für die Kompetenzunterschiede in pränumerischen wie im numerischen Bereich zwischen der Gruppe der erfolgreichen Kinder und der Gruppe der weniger erfolgreichen Kindern herauszufinden, ist das Item-Muster-Verfahren eine gute Hilfe, um erste Anhaltspunkte und grobe Hinweise zu liefern, die dann genauer untersucht werden müssen. Es werden bei diesem Verfahren einzelne Aufgaben, von denen man annehmen kann, dass sie aussagekräftig sind, herausgegriffen, die Ergebnisse werden in einer Tabelle zusammengefasst und das Ergebnismuster sollte dann Hinweise auf Lösungsstrukturen liefern. In diesem Abschnitt werden die Aufgaben A10, A13, A14, A19, A21, A23, A25, A28, A30, A31 und A37 für ein Item-Muster-Verfahren ausgewählt. Die Begründung, warum gerade diese Aufgaben für eine nähere Untersuchung ausgesucht wurden, werde ich dann geben, wenn ich die Aufgaben zum ersten Mal für eine genauere Untersuchung heranziehe und einzeln nochmals vorstelle. Eine Ausnahme möchte ich für die numerischen Aufgaben machen und eine Übersichtsbegründung geben.

Damit ein Kind über eine gefestigte Zählkompetenz verfügt, müssen verschiedene Faktoren zusammenspielen. Basierend auf Arbeiten von Fuson (1982) werden auf der Basis der Prinzipien-Nachher-Theorie (siehe ab Seite 50) fünf Phasen der Ausdifferenzierung der Zahlwortfolge unterschieden. Im Folgenden habe ich versucht, für diese Phasen repräsentative Aufgaben heranzuziehen, um so vielleicht Unterschiede in der Entwicklung der Zählkompetenz herauszufinden. Für die Phase der noch nicht differenzierten Ganzheitsauffassung der Zahlwortfolge (Phase I) eignet sich natürlich die Aufgabe A21, die Phase der differenzierten Ganzheitsauffassung der Zahlwortfolge (Phase II) wird durch die Aufgaben A28 (Auszählen) und die Aufgabe A31 (Abzählen) abgedeckt. Die Phase des Weiterzählen-Könnens (Phase III) wird durch die Aufgabe A23, die Phase der Auffassung der Zahlwörter als zählbare Einheiten (Phase IV) durch die Aufgabe A25 repräsentiert und das Subtrahieren in derselben Phase durch die Aufgabe A37 und zu guter Letzt wird die Phase der „zweiseitigen Durchlaufbarkeit“ der Zahlwortfolge (Phase V) durch die Aufgabe A30 abgedeckt. Durchläuft ein Kind alle diese fünf Phasen, verfügt es über eine gefestigte Zählkompetenz, die eine solide Grundlage für erfolgreiche Bewältigung kommender numerischer Probleme in verschiedensten Lebensabschnitten darstellt, u.a. auch die Zeit des Mathematikunterrichtes und den Einstieg in die Berufswelt. 
Um eine gewisse Trennschärfe zu erreichen, bildete ich drei Gruppen mit zweimal 26 und einmal 18 Kindern: In der Gruppe GI sind die 26 im Gesamttest Erfolgreichsten, in der Gruppe GII die 26 Durchschnittlichen und in der Gruppe GIII die 18 am wenigsten Erfolgreichen. Die entsprechenden Tabellen sind im Anhang ab Seite 270 aufgeführt. Ich habe nun auf der Grundlage der Item-Muster-Tabelle jede der ausgewählten Aufgaben mit allen andern Aufgaben der Item-Muster-Tabelle folgendermaßen in Beziehung gebracht:

\begin{tabular}{|l|c|}
\hline Beschreibung & Symbol \\
\hline Basisaufgabe gelöst - Vergleichaufgabe gelöst & $1-1$ \\
\hline Basisaufgabe gelöst - Vergleichaufgabe nicht gelöst & $1-0$ \\
\hline Basisaufgabe nicht gelöst - Vergleichaufgabe gelöst & 0 - 1 \\
\hline Basisaufgabe nicht gelöst - Vergleichaufgabe nicht gelöst & 0 - 0 \\
\hline
\end{tabular}

Tab. 32 Item-Muster Auswertungstabelle

Die Aufteilung in drei Leistungsgruppen hat sich als ergiebig herausgestellt, da so einige Auffälligkeiten zu Tage traten. Nicht ganz unerwartet stellt man fest, dass die Gruppe GIII grundsätzlich überfordert ist. Der überwiegende Anteil dieser 18 Kinder war nicht in der Lage, überhaupt eine der vorliegenden Aufgaben zu lösen. Es ist darum nicht verwunderlich, dass es insbesondere in dieser schwächsten Gruppe GIII sehr schwer war, mit Hilfe von Tabellen Aussagen über mathematische Kompetenzen machen zu können. Die Gruppe GII ist für eine Vergleichsauswertung weitaus lohnender, da die Ergebnisse oft schön auf die Ausfälle 1-1, 1-0, 0-1 und 0-0 verteilt sind. Die Gruppe GI hat nach Voraussetzung natürlich wenig bis keine Probleme mit der Lösung der vorliegenden Aufgaben. Trotzdem ist häufig eine für die Auswertung brauchbare Verteilung der Ausfälle feststellbar. Sehr oft war da der Vergleich der Gruppe GI mit hohem Kompetenzniveau mit der Gruppe GII mit durchschnittlichem Kompetenzniveau aussagekräftiger als ein Vergleich mit der Gruppe GIII, da wie bereits erwähnt, die Kinder dieser Gruppe mehrmalig keine der vorliegenden Aufgaben lösten.

Im Folgenden gebe ich Auffälligkeiten wieder, die uns auch bei den folgenden individuellen Betrachtungen über Lösungsverhalten nützlich sein könnten. 


\subsubsection{Aufgabengruppe A10 $\rightarrow$ Ann}

Zur Bestimmung der Trennschärfe wähle ich die Vierfeldertafel in modifizierter Form, dabei ist die 1-1 - Beziehung dem $a$, die 1-0 - Beziehung dem $b$, die 0-1 - Beziehung $\operatorname{dem} c$ und die 0-0 - Beziehung dem $d$ in der für die Berechnung der Trennschärfe benutzten Formel (siehe Seite 144) einzusetzen. Für eine saubere Bestimmung dieses Korrelationskoeffizienten benutze ich vor allem die Spalte „Alle“, bei der alle Ergebnisse zusammengefasst sind. Als Außenkriterium ist „Löst die Aufgabe A10“ / „Löst die Aufgabe A10 nicht“" gegeben. Zur Erinnerung seien alle neu auftretenden Aufgaben nochmals wiedergegeben.

Die Basisaufgabe A10 (siehe Seite 110): Hier siehst du einen Apfel mit Stiel, ohne Blatt und mit einem Würmchen, das aus dem Apfel herauskommt. [Ex. Zeigt auf den Apfel in dem Kasten oben links auf dem Blatt.] Zeige auf alle Äpfel, die genau gleich aussehen. Diese Aufgabe ist eine typische multiple Klassifizierungsaufgabe mit drei Merkmalsausprägungen und stellt sehr hohe Ansprüche an die Konzentrationsfähigkeit und Merkfähigkeit der Kinder. Die Aufgabe A10 haben gesamthaft 38 Kinder gelöst; in der Gruppe GI ist das Verhältnis von A10 - Löser zu A10 - Nichtlöser 20 zu 6; in der Gruppe GII 14 zu 12 und in der Gruppe GIII 4 zu 14.

a) $\mathrm{A} 10 \rightarrow \mathrm{A} 13$

\begin{tabular}{|c|c|c|c|c|}
\hline & GI & GII & GIII & Alle \\
\hline $1-1$ & 18 & 11 & 0 & 29 \\
\hline $1-0$ & 2 & 3 & 4 & 9 \\
\hline $0-1$ & 5 & 7 & 2 & 14 \\
\hline $0-0$ & 1 & 5 & 12 & 18 \\
\hline & & & & $r=0.33^{45}$ \\
\hline
\end{tabular}

Tab. 33 Item-Musterergebnis A10 $\rightarrow$ A13

Aufgabe A13 (siehe Seite 113): [Ex. gibt dem Kind ein Arbeitsblatt und einen Bleistift.] Hier siehst du Kerzenhalter. In jedem Kerzenhalter sollen Kerzen gesteckt werden. Zeichne Linien von den Kerzen zu den passenden Kerzenhaltern. In Anbetracht der kleinen Anzahlen sowohl der Kerzen wie auch der entsprechenden Kerzenfassungen ist diese Aufgabe im späteren Vergleich mit der Aufgabe A14 interes-

\footnotetext{
${ }^{45} r$ ist der Korrelationskoeffizient bezogen auf die darüber liegende Spalte „Alle“
} 
sant, da dann die Anzahlen viel größer sind. Wie verhält sich ein Kind bei kleineren Anzahlen? Wie bei größeren? Zählt es aus oder lässt es sich vom äußeren Anschein leiten? Diese Fragen werden dann im nächsten Kapitel 9 bei der individuellen Würdigung der Kinder genauer unter die Lupe genommen.

Von den 38 Kindern, die die Aufgabe A10 lösten, waren 9 nicht in der Lage, die Aufgabe A13 zu lösen. Bemerkenswert ist die Tatsache, dass doch 14 Kinder diese Aufgabe A13 gelöst haben obwohl sie die Aufgabe A10 nicht schafften. Dieser Anteil der 0-1 - Löser ist relativ hoch (20\%), was den Schluss zulässt, dass die komplexe Klassifizierungsaufgabe A10 nicht Grundvoraussetzung für das Lösen der Zuordnungsaufgabe A13 ist, obschon doch 29 Kinder A10 als auch A13 lösten. Der relativ hohe Korrelationskoeffizient $r=0.33$ besagt, dass die Aufgabe A13 von den Kindern, die die Aufgabe A10 erfolgreich lösten, eher besser gelöst wird als von den Kindern, die die Aufgabe A10 nicht erfolgreich gelöst haben.

b) $\mathrm{A} 10 \rightarrow \mathrm{A} 14$

\begin{tabular}{|c|c|c|c|c|}
\cline { 2 - 5 } \multicolumn{1}{c|}{} & GI & GII & GIII & Alle \\
\hline $1-1$ & 13 & 3 & 0 & 16 \\
\hline $1-0$ & 7 & 11 & 4 & 22 \\
\hline $0-1$ & 4 & 5 & 1 & 10 \\
\hline $0-0$ & 2 & 7 & 13 & 22 \\
\hline \multicolumn{1}{r|}{} & $r=0.11$ \\
\hline
\end{tabular}

Tab. 34 Item-Musterergebnis A10 $\rightarrow$ A14

Aufgabe A14 (siehe Seite 114): [Ex. gibt dem Kind ein Arbeitsblatt und einen Bleistift.] Hier siehst du drei Bilder mit Hühnern und Eiern. Kannst du das Bild finden, in dem jedes Huhn jeweils ein Ei gelegt hat? Du darfst Linien zeichnen. Wie bereits erwähnt, ist bei dieser Aufgabe die Anzahl der zu betrachtenden Objekte weitaus größer als bei der vorigen Aufgabe. Eine vernünftige Art, die Aufgabe zu lösen wäre es nun tatsächlich zwischen den Hühnern und den Eiern Zuordnungsstriche zu zeichnen und dann entsprechende Schlüsse zu ziehen. Eine andere Art wäre das Auszählen und eine dritte Möglichkeit wäre der bloße Augenschein. Im Kapitel 9 werden die Beobachtungen dargestellt und mit anderen Aufgaben verglichen, insbesondere mit der vorhergehenden Aufgabe A13. 
Ganz allgemein sei an dieser Stelle vermerkt, dass bei den folgenden Analysen vor allem die Ergebnisse der 1-0 und der 0-1 von Interesse sind. Die übrigen Ausfälle 11 und 0-0 werden meistens von einer großen Anzahl von Kindern belegt und geben eigentlich wenig Informationen für die betrachteten jeweiligen Beziehungen.

Die Aufgabe A14 ist nach der Kompetenzskala (siehe Seite 87) leicht schwerer als die Aufgabe A13, was unsere eigenen Untersuchungen bestätigen und trotzdem haben 10 Kinder A14 gelöst, ohne die Aufgabe A10 bewältigt zu haben. Zusammen mit dem sehr tiefen Korrelationskoeffizienten von $r=0.11$ deutet dies darauf hin, dass doch kein Zusammenhang zwischen der Aufgabe A10 und A14 besteht. Obwohl die beiden Aufgabe A13 und A14 vergleichbare Strukturen aufweisen, sind die Korrelationskoeffizienten doch sehr unterschiedlich, was wohl bedeutet, dass die Aufgabe A10 auf diese zwei Aufgaben unterschiedlich wirkt.

c) $\mathrm{A} 10 \rightarrow \mathrm{A} 19$

\begin{tabular}{|c|c|c|c|c|}
\cline { 2 - 5 } \multicolumn{1}{c|}{} & GI & GII & GIII & Alle \\
\hline $1-1$ & 15 & 7 & 1 & 23 \\
\hline $1-0$ & 5 & 7 & 3 & 15 \\
\hline $0-1$ & 3 & 3 & 2 & 8 \\
\hline $0-0$ & 3 & 9 & 12 & 24 \\
\hline \multicolumn{4}{r|}{} & $r=0.36$ \\
\cline { 2 - 4 }
\end{tabular}

Tab. 35 Item-Musterergebnis A10 $\rightarrow$ A19

Aufgabe A19 (siehe Seite 117): [Ex. gibt dem Kind ein Arbeitsblatt und einen Bleistift.] Hier siehst du Hunde. Jeder Hund soll einen Stock bekommen. Ein großer Hund bekommt einen großen Stock und ein kleiner Hund bekommt einen kleinen Stock. Zeichne Linien von den Hunden zu den Stöcken, die sie bekommen. Für eine Analyse schien es mir nötig, eine Reihenfolgeaufgabe zu berücksichtigen. Wie bei den Aufgaben A13 und A14 können die Kinder zur Lösung der Aufgabe Linien zeichnen. Die Gleichartigkeit dieser Lösungsstrategie könnte dann für eine spätere Beobachtungsanalyse förderlich sein.

Hier scheint es nützlich (und eher dienlich), die Aufgabe A10 zu können, um die Aufgabe A19 zu bewältigen; der relativ hohe Korrelationskoeffizient $r=0.36$ und die Tatsache, dass es nur 8 0-1 - Löser gibt, bestärkt diese Vermutung. Bei der Aufgabe A19 geht es ja auch nicht um ein bloßes Abzählen, sondern es sind schon meh- 
rere Kompetenzen gleichzeitig verlangt, nämlich einerseits die Größenvergleiche durchzuführen und andererseits die Bewertung der Vergleiche auszuführen, ganz ähnlich wie bei der Aufgabe A10.

d) $\mathrm{A} 10 \rightarrow \mathrm{A} 21$

\begin{tabular}{|c|c|c|c|c|}
\cline { 2 - 5 } \multicolumn{1}{c|}{} & GI & GII & GIII & Alle \\
\hline $1-1$ & 19 & 6 & 0 & 25 \\
\hline $1-0$ & 1 & 8 & 4 & 13 \\
\hline $0-1$ & 5 & 7 & 1 & 13 \\
\hline $0-0$ & 1 & 5 & 13 & 19 \\
\hline \multicolumn{2}{r|}{}
\end{tabular}

Tab. 36 Item-Musterergebnis A10 $\rightarrow$ A21

Aufgabe A21 (siehe Seite 120): Zähle bis 20.

Die erste Phase auf dem Weg zur Erlangung einer soliden Zählkompetenz ist nach der Prinzipien-Nachher-Theorie bei den allermeisten von uns interviewten Kindern erfolgreich durchlaufen worden. Die Aufgabe stellt noch keine weiteren Ansprüche als das einfache Aufsagen der Zahlwörter bis 20. Für fast alle Kinder ist die Zahlreihe schon weit mehr als nur ein Aufsagen eines noch undifferenzierten Ganzen, ähnlich einem auswendig gelerntem Gedicht oder Sprüchlein, selbst wenn die Ergebnisse nicht bei allen Kindern überragend sind. Es gilt zu berücksichtigen, dass nur die Kinder, die die Zahl 20 auch tatsächlich erreichten, die Aufgabe erfolgreich gelöst haben. Viele Kinder, die keinen Punkt erhielten, haben trotzdem die Phase I erfolgreich bewältigt, d.h. sie sind sehr wohl in der Lage, die Zahlwörter in der Zahlwortreihe zu differenzieren, schafften aber aus unterschiedlichen Gründen die Reihe bis 20 nicht. Wir werden im nächsten Kapitel 9 sehen, dass es Kinder gibt, die das Aufsagen der Zahlwortreihe bis 20 nicht schaffen, obwohl sie sehr wohl in der Lage sind, eine Kollektion von 20 Gegenständen erfolgreich abzuzählen.

Generell sei an dieser Stelle nochmals festgehalten, dass die Gruppe GIII in allen untersuchten Kombinationen überfordert war. Diese Erkenntnis ist insofern von Bedeutung, dass jetzt feststeht, dass Kinder mit tiefem Kompetenzniveau auf allen untersuchten Gebieten unterdurchschnittliche Ergebnisse liefern. Der Entwicklungsstand dieser Gruppe von Kindern ist offenbar noch nicht dergestalt, dass er die Lösung der vorliegenden zum Teil schon sehr komplexen Aufgaben zulässt. Das Al- 
ter dieser Gruppe GIII ist im Durchschnitt nur leicht tiefer als das der Gruppen GII aber beträchtlich tiefer als das der Gruppe GI (siehe Tab. 37). Außerdem ist die Gruppe GIII auf das Alter bezogen heterogen, sind doch in dieser Gruppe GIII 2 Kinder (von 18) aus der Alterstufen III und 4 Kinder aus der Alterstufe IV. Zwar lässt sich sagen, dass der doch große Altersunterschied zwischen den Gruppen GI und GIII von 10 Monaten mitverantwortlich ist für die großen Leistungsunterschiede dieser beiden Gruppen GI und GIII. Der Altersunterschied zwischen den Gruppen GII und GIII ist aber zu vernachlässigen und trotzdem sind die Leistungen der Gruppe GIII weit geringer als die der Gruppe GII. Diese Tatsache bestärkt zum wiederholten Male die These, dass nicht in erster Linie das Alter für hohe Leistungsfähigkeit verantwortlich ist, sondern der allgemeine Entwicklungsstand des Kindes, der ja bekannterweise altersmäßig äußerst individuell abläuft.

\begin{tabular}{|c|c|c|c|}
\hline & GI & GII & GIII \\
\hline Altersgruppendurchschnitt & $5 ; 12^{46}$ & $5 ; 4$ & $5 ; 2$ \\
\hline
\end{tabular}

Tab. 37 Durchschnittsalter der drei Gruppen

Die vorliegende Kombination A10 $\rightarrow$ A21 ist von großem Interesse, legt sie doch die Beziehung Klassifizierung - Zählkompetenz offen. Sind 25 Kinder in der Lage, beide Aufgaben A10 und A21 zu lösen, ist der Anteil der 1-0 - Löser und der 0-1 Löser mit 13 Kindern gleichgroß, des weiteren lösen 19 Kinder weder Aufgabe A10 noch Aufgabe A21. Die beiden gleichgroßen Anteile der 1-0 - Löser und der 0-1 Löser mit je 13 Kindern (37\%) lässt auf einen geringen Zusammenhang dieser beiden Aufgaben schließen; es ist vollkommen unwichtig für das Lösen der Aufgabe A21, ob man die Aufgabe A10 bewältigt hat oder nicht. Hier bestätigt sich die Aussage des Kapitels 8.2.2 (ab Seite 145), dass Klassifizierungskonzepte und Zählkompetenz in keinem großen Zusammenhang stehen. Der Korrelationskoeffizient $r=0.25$ ist ebenfalls tief und zeigt tendenziell auf, dass erfolgreiche A10 - Löser die Zählaufgabe A21 nicht wesentlich besser lösen als die Kinder, die die Aufgabe A10 nicht gelöst haben. Sehr deutlich wird dieser Sachverhalt bei Betrachtung der Gruppe GII, wo die Verteilung sehr ausgeglichen

${ }^{46}$ Zur Erinnerung: 5;12 bedeutet 5 Jahre und 12 Monate. 
ist und der Korrelationsindex sogar ne-

gativ wird. $\left(r=-0.15^{47}\right)$

e) $\mathrm{A} 10 \rightarrow \mathrm{A} 28$

\begin{tabular}{|c|c|c|c|c|}
\cline { 2 - 5 } \multicolumn{1}{c|}{} & GI & GII & GIII & Alle \\
\hline $1-1$ & 16 & 5 & 0 & 19 \\
\hline $1-0$ & 4 & 9 & 4 & 19 \\
\hline $0-1$ & 4 & 4 & 1 & 9 \\
\hline $0-0$ & 2 & 8 & 13 & 23 \\
\hline \multicolumn{4}{r|}{}
\end{tabular}

Tab. 38 Item-Musterergebnis A10 $\rightarrow$ A28

Aufgabe A28 (siehe Seite 125): [Ex. legt 20 Holzwürfel in einem Haufen mit etwas Abstand zwischen den Holzwürfeln auf den Tisch.) Zähle diese Holzwürfel. (Das Kind darf auf die Holzwürfel zeigen oder sie während des Zählens zur Seite schieben.)

In der Phase II der Prinzipien-Nachher-Theorie gelingt u.a. das erfolgreiche Auszählen einer gegebenen Anzahl Gegenstände und genau dieses Problem muss in der Aufgabe A28 gelöst werden.

Gerade die Hälfte der 38 A10 - Löser haben auch die Auszählung der Kollektion von 20 Gegenständen geschafft. (Bei dieser Aufgabe wird sich zeigen, dass - wie schon mehrfach erwähnt - einige Kinder das Zählen von konkreten Gegenständen besser bewältigen als das bloße Aufsagen einer Zahlenreihe über die 10 hinaus.) Die vorliegenden Daten lassen keinen Zusammenhang zwischen der Klassifizierungsaufgabe A10 und dieser strukturierten Zählaufgabe A28 erkennen. Der Trennschärfekoeffizient $r=0.27$ ist relativ tief und etwa gleich wie bei der Aufgabenkombination $\mathrm{A} 10 \rightarrow \mathrm{A} 21 \quad(r=0.25)$, was den engen Zusammenhang der beiden Aufgabenkombinationen aufzeigt. Außerdem zeigt dies, dass erfolgreiche A10 Löser die Zählaufgabe A28 nicht wesentlich besser lösen als die Kinder, die die Aufgabe A10 nicht gelöst haben. Auch hier wird dieser Sachverhalt sehr deutlich bei der Betrachtung der Gruppe GII, wo der Korrelationsindex praktisch Null wird $(r=0.02)$.

\footnotetext{
${ }^{47}$ Ein negativer Korrelationskoeffizient zeigt an, dass die Aufgabe von der „Oberen Gruppe“ schlechter gelöst wird als von der „Unteren Gruppe“, und genau das ist innerhalb der Gruppe GII der Fall.
} 
f) $\mathrm{A} 10 \rightarrow \mathrm{A} 31$

\begin{tabular}{|c|c|c|c|c|}
\cline { 2 - 5 } \multicolumn{1}{c|}{} & GI & GII & GIII & Alle \\
\hline $1-1$ & 14 & 4 & 0 & 18 \\
\hline $1-0$ & 6 & 10 & 4 & 20 \\
\hline $0-1$ & 3 & 8 & 3 & 14 \\
\hline $0-0$ & 3 & 4 & 11 & 18 \\
\hline \multicolumn{1}{r|}{} & $r=0.04$ \\
\cline { 2 - 4 }
\end{tabular}

Tab. 39 Item-Musterergebnis A10 $\rightarrow$ A31

Aufgabe A31 (siehe Seite 128):[Ex. gibt dem Kind 15 Holzwürfel.] Lege eine Reihe mit elf Holzwürfeln.

In der zweiten Phase der Prinzipien-Nachher-Theorie wird neben der Kompetenz des Auszählens auch diejenige des Abzählens erlangt. Die Kinder müssen durch Zählen eine Kollektion einer bestimmten Anzahl Gegenstände herstellen.

Der Korrelationskoeffizient $r=0.04$ zeigt, dass das Abzählen in keiner Weise von Klassifizierungskompetenzen abhängt, es spielt also für das Lösen der Aufgabe A31 überhaupt keine Rolle, ob die Aufgabe A10 richtig oder falsch gelöst wurde. Die 20 1-0 - Löser und die 14 0-1 - Löser sind ebenfalls Beleg dafür, dass die multiple Klassifikation keinen Einfluss auf das Abzählen ausübt.

g) $\mathrm{A} 10 \rightarrow \mathrm{A} 23$

\begin{tabular}{|c|c|c|c|c|}
\cline { 2 - 5 } \multicolumn{1}{c|}{} & GI & GII & GIII & Alle \\
\hline $1-1$ & 15 & 6 & 0 & 21 \\
\hline $1-0$ & 5 & 8 & 4 & 17 \\
\hline $0-1$ & 4 & 7 & 1 & 12 \\
\hline $0-0$ & 2 & 5 & 13 & 20 \\
\hline
\end{tabular}

Tab. 40 Item-Musterergebnis A10 $\rightarrow$ A23

Aufgabe A23 (siehe Seite 121): „Zähle weiter von neun bis fünfzehn: sechs, sieben, acht ... “

Nach der Prinzipien-Nachher-Theorie Phase III deckt die gewählte Aufgabe exakt das „Weiterzählen-Können“ ab: ein Start bei „1“ ist nicht mehr unbedingt nötig, es 
kann auch von $a$ nach $b$ gezählt werden. In dieser Phase wird das Weiterzählen resp. das Rückwärtszählen auch zur kardinalen Addition resp. Subtraktion benutzt.

Die Kompetenz in multipler Klassifikation bietet keine Unterstützung beim Lösen dieser wichtigen Aufgabe: die 17 1-0 - Löser und insbesondere die 12 0-1 - Löser weisen auf diesen Tatbestand hin. Der tiefe Korrelationskoeffizient $r=0.18$ zeigt, dass es fast keinen Unterschied zwischen guten und weniger guten A10 - Lösern bei der Bewältigung der Aufgabe A23 gibt.

h) $\mathrm{A} 10 \rightarrow \mathrm{A} 25$

\begin{tabular}{|c|c|c|c|c|}
\cline { 2 - 5 } \multicolumn{1}{c|}{} & GI & GII & GIII & Alle \\
\hline $1-1$ & 12 & 1 & 0 & 13 \\
\hline $1-0$ & 8 & 13 & 4 & 25 \\
\hline $0-1$ & 1 & 1 & 0 & 2 \\
\hline $0-0$ & 5 & 11 & 14 & 30 \\
\hline \multicolumn{2}{r|}{} & \multicolumn{1}{r|}{$r=0.34$} \\
\hline
\end{tabular}

Tab. 41 Item-Musterergebnis A10 $\rightarrow$ A25

Aufgabe A25 (siehe Seite 122): Zähle bis vierzehn und überspringe immer jeweils eine Zahl: zwei, vier, sechs ...

In der nach der Prinzipien-Nachher-Theorie folgenden Phase IV werden die Zahlwörter nicht nur als wohlunterscheidbare Einheiten aufgefasst, sondern selbst als zählbare Einheiten begriffen, so dass in diesem Entwicklungsstadium auch Teilstücke ausgezählt werden können, insbesondere gelingt das Weiterzählen von $a$ nach $b$ um $n$ Einheiten.

Von den 15 Kindern, die die Aufgabe A25 lösten, haben 13 auch die Aufgabe A10 erfolgreich bewältigt und 25 Kinder haben wohl die Aufgabe A10 gelöst, scheiterten aber bei der Aufgabe A25, nur zwei Kinder lösten die Aufgabe A25 ohne A10 bewältigt zu haben. Diese Zahlen lassen darauf schließen, dass das Lösen dieser Klassifizierungsaufgabe A10 eine notwendige Bedingung für das Lösen der Aufgabe A25 darstellt. Wenn man die Aufgabe A10 genauer betrachtet, fällt auf, dass eine Klassifizierung mit drei Merkmalsausprägungen verlangt wird (mit Stiel, ohne Blatt, mit Würmchen). Bei der Aufgabe A25 ${ }^{48}$ müssen ebenfalls mehrere Informationen gleichzeitig beachtet und verarbeitet werden: Das Kind muss als erstes erkennen,

\footnotetext{
${ }^{48}$ Die Aufgabe A25 hat den tiefen Schwierigkeitsindex 28.6; ist also eine eher schwere Aufgabe.
} 
dass der Interviewer immer eine Zahl auslässt, als zweites muss es dann selber die nächste Zahl sieben überspringen und acht nennen, dann zum dritten muss es so fortfahren, bis 14 erreicht wird, und zum vierten muss es sich daran erinnern, dass es bei der Zahl 14 aufhören muss. Die Trennschärfe ist mit $r=0.34$ wieder etwas höher; dabei muss aber beachtet werden, dass die Gruppen GII und GIII sich fast identisch verhalten $(r \approx 0.0)$ und darum nur die Gruppe GI für diese Trennschärfe verantwortlich zeichnet $\left(r_{\mathrm{GI}}=0.37\right)$.

i) $\mathrm{A} 10 \rightarrow \mathrm{A} 37$

\begin{tabular}{|c|c|c|c|c|}
\cline { 2 - 5 } \multicolumn{1}{c|}{} & GI & GII & GIII & Alle \\
\hline $1-1$ & 17 & 9 & 0 & 26 \\
\hline $1-0$ & 3 & 5 & 4 & 12 \\
\hline $0-1$ & 6 & 6 & 1 & 13 \\
\hline $0-0$ & 0 & 6 & 13 & 19 \\
\hline \multicolumn{1}{r}{} & & $r=0.28$ \\
\hline
\end{tabular}

Tab. 42 Item-Musterergebnis A10 $\rightarrow$ A37

Aufgabe A37 (siehe Seite 131): [Ex. zeigt auf das Bild mit 9 Murmeln.] Du hast neun Murmeln. Du verlierst drei Murmeln. Wie viele Murmeln hast du übrig? Zeige auf den Kasten mit der richtigen Anzahl von Murmeln. [Ex. zeigt auf die Reihe der Bilder unten auf dem Blatt.]

In der Phase IV der Prinzipien-Nachher-Theorie kommt es zur Sicherheit beim Addieren wie auch beim Subtrahieren. Da das Rückwärtszählen sich spät entwickelt (um das 7. Lebensjahr), sind Subtraktionsaufgaben von großem Interesse, insbesondere was die Lösungsstrategien anbelangt, auf die ich im Kapitel 9 eingehen werde. Bei dieser Kombination ist der Anteil der 1-1 - Löser mit 26 Kindern zwar sehr hoch; aber halt doch zu wenig markant, als dass sich ein Zusammenhang zwischen den Aufgaben A10 und A37 herauslesen ließe. Auffällig ist der fast identische Anteil der 1-0 - Löser und der 0-1 - Löser mit 12 resp. 13 Kindern, was darauf hinweist, dass das Lösen der Aufgabe A37 keiner Vorkenntnisse in Klassifizieren bedarf. Der Korrelationskoeffizient $r=0.28$ ist eher tief, was bedeutet, dass die A10 Löser keine wesentlich höhere Kompetenz beim Lösen der Aufgabe A37 an den Tag legen als die Kinder, die die Aufgabe A10 nicht gelöst haben. 
j) $\mathrm{A} 10 \rightarrow \mathrm{A} 30$

\begin{tabular}{|c|c|c|c|c|}
\cline { 2 - 5 } \multicolumn{1}{c|}{} & GI & GII & GIII & Alle \\
\hline $1-1$ & 8 & 0 & 0 & 8 \\
\hline $1-0$ & 12 & 14 & 4 & 30 \\
\hline $0-1$ & 2 & 1 & 0 & 3 \\
\hline $0-0$ & 4 & 11 & 14 & 29 \\
\hline
\end{tabular}

Tab. 43 Item-Musterergebnis A10 $\rightarrow$ A30

Aufgabe A30 (siehe Seite 126): [Ex. legt 17 Holzwürfel auf den Tisch in einer Reihe mit etwas Abstand zwischen den Holzwürfeln.] Hier siehst du siebzehn Holzwürfel. Zeige auf die Holzwürfel und zähle sie rückwärts.

In der letzten Phase V kann ein Kind mit Sicherheit und mit hoher Flexibilität die Zahlwortfolge von jeder Stelle aus und in jede Richtung durchlaufen.

In der Tendenz verhält sich diese Kombination A10/A30 wie die Kombination A10/A25: ein sehr hoher Anteil von 30 1-0 - Lösern steht einer kleinen Anzahl von 3 0-1 - Lösern gegenüber. Die Problemstruktur der beiden Aufgaben A10 und A30 ist sehr unterschiedlich und dies wirkt sich auch in der Trennschärfe aus. Im Gegensatz zur Aufgabenkombination A10 $\rightarrow$ A25 ist hier der Trennschärfekoeffizient mit $r=0.16$ tief, das heißt, dass die Guten gegenüber den weniger Guten bezüglich der Aufgabe A10 beim Rückwärtszählen nur ganz knapp besser sind.

\subsubsection{Zusammenfassung}

Als erstes sei nochmals festgehalten, dass das Lösen der Aufgabe A10 nur für 2 der 10 vorliegenden Aufgaben eine Voraussetzung zur Lösung sein könnte; es sind dies die Aufgabe A19 (eher schwacher Einfluss) und die Aufgabe A25 (etwas stärkerer Einfluss). Die übrigen 8 Aufgaben werden ohne erkennbare Einflussnahme der Aufgabe A10 gemeistert. Dies unterstreicht die auf Seite 146 geäußerte Feststellung, dass das Beherrschen von Klassifizierungsaufgaben im Allgemeinen auf Zählkompetenz keinen oder allenfalls nur einen sehr kleinen Einfluss ausübt.

Im Weiteren muss noch eine Antwort auf die Frage gesucht werden, ob es denn ein Hinweis auf verschiedenes Lösungsverhalten der einzelnen Leistungsgruppen gibt, die denn die beobachtbaren doch beträchtlichen Kompetenzunterschiede ausmachen. Aus 
den Analysebedingungen heraus liegt es auf der Hand, dass die Gruppe GI a priori bei allen Kombinationen die besten Resultate liefert.

Die in den Leistungsgruppen GI und GII fast überall feststellbaren tiefen (oft auch negativen) Korrelationskoeffizienten (siehe vollständige Tabellen im Anhang ab Seite 272) weisen unmissverständlich darauf hin, dass die meisten Aufgaben genau so gut oder so schlecht von den „Erfolgreichen“ wie von den „weniger Erfolgreichen“ bezüglich der Aufgabe A10 gelöst wurden: es sind keine bemerkenswerten Unterschiede zwischen den beiden Gruppen GI und GII auszumachen. Die Gruppe GIII gibt keinerlei Hinweise auf irgendwelche Lösungsstrukturen, da im Wesentlichen alle diese Kinder bei allen Kombinationen überfordert waren.

Fazit: Wie jetzt schon mehrfach erwähnt, übt das Beherrschen von (auch multiplen) Klassifizierungsproblemen keinen oder zumindest nur einen ganz kleinen Einfluss auf die Zählkompetenz aus.

\subsubsection{Aufgabengruppe A14 $\rightarrow$ Ann}

Da die Aufgaben A13 und A14 beide der Eins-zu-eins-Komponentengruppe angehören, unterziehe ich nur die Aufgabe A14 einer Korrelationsanalyse; die Aufgabe A13 wird dann im Kapitel 9 vor allem für Vergleiche mit der Aufgabe A14 herangezogen. Aus diesem Grund werde ich in diesem Abschnitt auch nicht auf die bereits erwähnten verschiedenen Lösungsmöglichkeiten der Aufgabe A14 eingehen.

Alle übrigen Aufgaben brauchen jetzt nicht mehr im Einzelnen vorgestellt zu werden, da im vorigen Abschnitt alle Aufgaben schon präsentiert wurden. Im Weiteren werde ich wegen der besseren Lesbarkeit die Darstellung der Bezugstabellen sehr unterschiedlich gestalten. Es werden nur noch diejenigen Tabellen vollständig dargestellt, die wesentliche Informationen liefern, ansonsten werde ich oft nur die interessanten 1-0 und 0-1 Beziehungen darstellen oder bei belanglosen Bezügen überhaupt nichts. Zur allgemeinen Übersicht sei noch erwähnt, dass die Anteile der 1-1 - Löser um 18 und die Anteile der 0-0 Löser um 30 schwanken. Es sei hier nochmals darauf hingewiesen, dass die vollständigen Tabellen im Anhang ab Seite 272 aufgeführt sind.

Die Aufgabe A14 haben gesamthaft 26 Kinder gelöst; in der Gruppe GI ist das Verhältnis von A14 - Löser zu A14 - Nichtlöser 17 zu 9; in der Gruppe GII 8 zu 18 und in der Gruppe GIII 1 zu 17. 
a) $\mathrm{A} 14 \rightarrow \mathrm{A} 19$

Der 0-1 Anteil ist mit 15 Kindern höher als der 1-0 Anteil mit 10 Kindern. Die Aufgabe A19 steht darum in keinem Bezug zur Aufgabe A14. Der relativ tiefe Korrelationskoeffizient $r=0.27$ deutet darauf hin, dass auch die A14 - Löser nicht wesentlich besser bei der Bewältigung der Aufgabe A19 abschneiden als die A14 Nichtlöser.

b) A14 $\rightarrow$ A21

\begin{tabular}{|c|c|c|c|c|}
\cline { 2 - 5 } \multicolumn{1}{c|}{} & GI & GII & GIII & Alle \\
\hline $1-1$ & 16 & 3 & 0 & 19 \\
\hline $1-0$ & 1 & 5 & 1 & 7 \\
\hline $0-1$ & 8 & 10 & 1 & 19 \\
\hline $0-0$ & 1 & 8 & 16 & 25 \\
\hline \multicolumn{4}{r|}{} & $r=0.29$ \\
\cline { 1 - 4 }
\end{tabular}

Tab. 44 Item-Musterergebnis A14 $\rightarrow$ A21

Hier ist der 0-1 Anteil mit 19 Kindern markant höher als der 1-0 Anteil mit nur 7 Kindern. Viele Kinder schaffen die klassische Zuordnungsaufgabe A14 nicht, dennoch sind sie durchaus in der Lage zu zählen. Obwohl der Schwierigkeitsindex der Aufgabe A14 mit 37.14 tiefer ist als derjenige der Aufgabe A21 mit 54.29, muss doch zur Kenntnis genommen werden, dass Eins-zu-eins-Zuordnung nicht zwingend für Zählkompetenz ist.

c) $\mathrm{A} 14 \rightarrow \mathrm{A} 28$

\begin{tabular}{|c|c|c|c|c|}
\cline { 2 - 5 } \multicolumn{1}{c|}{} & GI & GII & GIII & Alle \\
\hline $1-0$ & 2 & 5 & 1 & 8 \\
\hline $0-1$ & 5 & 6 & 1 & 12 \\
\hline & & & & $r=0.41$ \\
\cline { 2 - 4 }
\end{tabular}

Tab. 45 Item-Musterergebnis A14 $\rightarrow$ A28

Auch hier weisen die Zahlen mit 8 1-0 - Löser zu 12 0-1 - Löser auf Unabhängigkeit der Zählkompetenz von Zuordnungskompetenzen hin. Im Unterschied zum reinen Aufsagen der Zahlwortreihe kann man bei der Aufgabe A28 konkrete Materia- 
lien auszählen. Die Aufgabe A14 kann aber ebenfalls mit zählen gelöst werden, das heißt nun, dass die beiden Aufgabe in der Struktur ähnlich sind. Der hohe Korrelationskoeffizient $r=0.41$ lässt sich so gut erklären.

d) $\mathrm{A} 14 \rightarrow \mathrm{A} 31$

Bei dieser Kombination ist keine Besonderheit zu vermerken, außer dass ebenfalls die 0-1 - Löser gegenüber den 1-0 - Lösern mit 17 zu 11 in der Überzahl sind. Der Korrelationskoeffizient $r=0.18$ ist hingegen wieder tief, was bedeutet, dass wohl keine Abhängigkeit besteht.

e) $\mathrm{A} 14 \rightarrow \mathrm{A} 23$

\begin{tabular}{|c|c|c|c|c|}
\hline & GI & GII & GIII & Alle \\
\hline $1-0$ & 3 & 4 & 1 & 8 \\
\hline $0-1$ & 5 & 9 & 1 & 15 \\
\hline
\end{tabular}

Tab. 46 Item-Musterergebnis A14 $\rightarrow$ A23

Auch hier wird deutlich, dass das Lösen der vorliegenden Zuordnungsaufgabe A14 keine Voraussetzung für das fortschreitende Zählen darstellt. Wohl sind die guten A14 - Löser ein wenig besser beim Lösen der Aufgabe A23, aber als Ganzes lässt sich sagen, dass hier ebenfalls keine Kompetenzen der Eins-zu-eins-Zuordnung für erfolgreiches Weiterzählen nötig sind.

f) $\mathrm{A} 14 \rightarrow \mathrm{A} 25$

\begin{tabular}{|c|c|c|c|c|}
\cline { 2 - 5 } \multicolumn{1}{c|}{} & GI & GII & GIII & Alle \\
\hline $1-1$ & 9 & 1 & 0 & 10 \\
\hline $1-0$ & 8 & 7 & 1 & 16 \\
\hline $0-1$ & 4 & 1 & 3 & 8 \\
\hline $0-0$ & 5 & 17 & 14 & 36 \\
\hline \multicolumn{4}{r|}{} & $r=0.22$ \\
\hline
\end{tabular}

Tab. 47 Item-Musterergebnis A14 $\rightarrow$ A25

Zum ersten Mal bei den Kombinationen A14 $\rightarrow$ Ann ist der 1-0 Anteil mit 16 Kindern höher als der 0-1 Anteil mit 8 Kindern. Dies deutet aber nicht darauf hin, dass 
es für die Lösung der Aufgabe A25 günstig wäre, die Aufgabe A14 zu bewältigen; denn es ist zu beachten, dass der 0-1 Anteil (8 Kinder) fast so hoch wie der 1-1 Anteil (10 Kinder) ist. D. h. nun aber, dass unabhängig vom Lösen der Aufgabe A14 die schwere Aufgabe A25 gelöst werden kann.

g) $\mathrm{A} 14 \rightarrow \mathrm{A} 37$

\begin{tabular}{|c|c|c|c|c|}
\cline { 2 - 5 } \multicolumn{1}{c|}{} & GI & GII & GIII & Alle \\
\hline $1-0$ & 1 & 3 & 0 & 4 \\
\hline $0-1$ & 7 & 10 & 0 & 17 \\
\hline \multicolumn{4}{r|}{} & $r=0.45$ \\
\cline { 2 - 5 }
\end{tabular}

Tab. 48 Item-Musterergebnis A14 $\rightarrow$ A37

Diese Beziehung ist durch den hohen Korrelationskoeffizienten $r=0.45$ von Interesse: die guten A14 - Löser bewältigen die Aufgabe A37 mit weit höherer Wahrscheinlichkeit als die andern. Dieser Aspekt ist wohl eher durch die allgemeine Leistungsfähigkeit zu erklären, denn es gibt nur 4 1-0 - Löser gegenüber 17 0-1 Lösern. Das bedeutet, dass der Einfluss der Aufgabe A14 auf das Lösen der Subtraktionsaufgabe A37 sehr gering sein muss.

h) $\mathrm{A} 14 \rightarrow \mathrm{A} 30$

Der Schwierigkeitsindex der Aufgabe A30 ist bei unserer Untersuchung 15.71, was bedeutet, dass diese Aufgabe sehr schwer ist. Von den 26 A14 - Lösern schafften nur gerade 8 die Aufgabe A30. Dies ist wiederum ein Indiz dafür, dass die Eins-zueins-Zuordnungen wohl nicht abträglich für hohe Zählkompetenz sind, aber wohl auch nicht so zentral in der Bedeutung, wie gemeinhin vermutet wird.

\subsubsection{Zusammenfassung}

Die Ergebnisse der Datenanalyse zeigen deutlich, dass die im vorigen Kapitel geäußerte Erkenntnis, wonach das Beherrschen von Eins-zu-eins-Zuordnungsaufgaben im Allgemeinen auf Zählkompetenz nur einen sehr kleinen Einfluss ausübt, untermauert wird. 
Die Korrelationskoeffizienten sind denn auch mit Ausnahme der Aufgabenkombinationen A14 $\rightarrow$ A28 und A14 $\rightarrow$ A37 überall sehr tief. Die Aufgaben A14 und A28 sind wie bereits erwähnt strukturell ähnlich, was den hohen Trennschärfekoeffizienten erklärt. Die allgemeine Leistungsfähigkeit muss wie gesehen für diese hohe Trennschärfe bei der Aufgabe A37 verantwortlich gemacht werden.

Die einzelnen Leistungsgruppen unterscheiden sich im Lösungsverhalten insofern, als die Gruppe GIII wie immer überfordert war und die Gruppen GII und GI oft unterschiedliche Korrelationskoeffizienten aufweisen (siehe Tabellen im Anhang ab Seite 272). Ansonsten sind keine bemerkenswerten Unterschiede zwischen den beiden Gruppen GI und GII auszumachen.

Fazit: Wie schon mehrfach beschrieben, übt das Beherrschen von Eins-zu-einsZuordnungsaufgaben einen weit geringeren Einfluss auf die Zählkompetenz aus, als gemeinhin angenommen wurde.

\subsubsection{Aufgabengruppe A19 $\rightarrow$ Ann}

Die wohl offensichtlichste Form einer Reihenfolge ist die Folge der natürlichen Zahlen. Es verwundert darum nicht, dass die Kompetenz von allgemeinen Reihenfolgenbildungen als die Voraussetzung für die Erlangung und Beherrschung der Zahlwortfolge betrachtet wird. Die folgende Analyse der Ergebnisse der Schweizer Untersuchung stellt diese Ansicht aber in Frage.

Die Aufgabe A19 haben gesamthaft 31 Kinder gelöst; in der Gruppe GI ist das Verhältnis von A19 - Löser zu A19 - Nichtlöser 18 zu 8; in der Gruppe GII 10 zu 16 und in der Gruppe GIII 3 zu 15. Die Tabellendarstellung werde ich entsprechend dem Informationsgehalt wie im vorigen Abschnitt sehr unterschiedlich handhaben. Die Anteile der 1-1 - Löser und der 0-0 - Löser bewegen sich bei den vorliegenden Aufgabenkombinationen um die 20 Kinder und sind meistens nicht von großem Interesse.

a) $\mathrm{A} 19 \rightarrow \mathrm{A} 21$

\begin{tabular}{|c|c|c|c|c|}
\cline { 2 - 5 } \multicolumn{1}{c|}{} & GI & GII & GIII & Alle \\
\hline $1-1$ & 17 & 2 & 0 & 19 \\
\hline $1-0$ & 1 & 8 & 3 & 12 \\
\hline $0-1$ & 7 & 11 & 1 & 19 \\
\hline
\end{tabular}




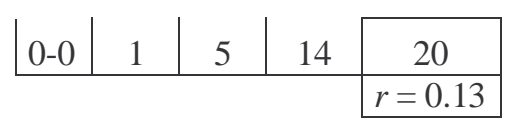

Tab. 49 Item-Musterergebnis A19 $\rightarrow$ A21

Dass bei der vorliegenden Untersuchung die Zählkompetenz nicht von der Reihenfolgenbildung abhängig ist, zeigt diese Tabelle in eindrucksvoller Art und Weise. Es wird offensichtlich, dass die Bewältigung der Aufgabe A19 keinen Einfluss auf das reine Aufsagen der Zahlwortreihe bis 20 ausübt; denn den 12 1-0 Lösern stehen 19 0-1 - Löser gegenüber. Der sehr niedrige Korrelationskoeffizient $r$ $=0.13$ dokumentiert die Belanglosigkeit der Gruppenzugehörigkeit ${ }^{49}$ für erfolgreiches Bewältigung der Zahlwortreihe, d.h. dass die A19 - Löser beim Zählen bis 20 gegenüber den A19 - Nichtlösern nicht bevorteilt sind.

b) $\mathrm{A} 19 \rightarrow \mathrm{A} 28$

Fast identisch präsentiert sich die Lage bei der Aufgabe A28, die ja auch das reine Zählen als Basiskompetenz verlangt. Mit 15 resp. 14 Kindern sind die beiden Anteile der 1-0 und 0-1 - Löser fast identisch. Der niedrige Trennschärfekoeffizient $r=$ 0.16 weist wieder darauf hin, dass die Gruppenzugehörigkeit keine hohe Lösungswahrscheinlichkeit garantiert, sodass auch hier wieder der Schluss gezogen werden darf, dass Reihenfolgenbildung für hohe Zählkompetenz nicht zwingend notwendig ist.

c) $\mathrm{A} 19 \rightarrow \mathrm{A} 31$

Mit fast identischen Daten zur soeben besprochenen Aufgabenkombination präsentiert sich die vorliegende. Die 1-0 und 0-1 Anteile sind hier 15 und 16 und der Korrelationskoeffizient von $r=0.11$ ergänzt die oben gemachten Beobachtungen und bestätigt die entsprechenden Schlüsse.

d) A19 $\rightarrow$ A23

\begin{tabular}{|c|c|c|c|c|}
\cline { 2 - 5 } \multicolumn{1}{c|}{} & GI & GII & GIII & Alle \\
\hline $1-0$ & 4 & 6 & 3 & 13 \\
\hline $0-1$ & 5 & 9 & 1 & 15 \\
\hline
\end{tabular}

\footnotetext{
${ }^{49}$ Ganz entsprechend der Vierfeldertafel gibt es hier eine obere Gruppe (die Erfolgsgruppe, hier die 1-1 und 1-0 - Löser) und eine untere Gruppe (die Misserfolgsgruppe, hier die 0-1 und die 0-0 - Löser).
} 
Tab. 50 Item-Musterergebnis A19 $\rightarrow$ A23

Eine solide Reihenfolgenbildungskompetenz befähigt die Kinder, jederzeit eine Reihe von einem beliebigen Ort in der Reihe nach einem vorgegebenen Bildungsgesetz weiterzuentwickeln. Das Weiterzählen-Können ist nun genau solch eine beschriebene Situation und man könnte jetzt davon ausgehen, dass Kinder in der oberen Gruppe das Weiterzählen beherrschen, die Kinder in der unteren Gruppe nicht: den 13 1-0 -Lösern stehen nun aber 15 0-1 - Löser gegenüber, wobei noch auffällt, dass sich die Gruppen GI und GII sehr ähnlich verhalten. Der Korrelationskoeffizient $r=0.20$ ist wieder relativ tief und es scheint sich also auch hier zu bewahrheiten, dass die Reihenfolgenbildung einen kleineren Einfluss auf Zählkompetenz ausübt, als gemeinhin angenommen wird.

e) $\mathrm{A} 19 \rightarrow \mathrm{A} 25$

\begin{tabular}{|c|c|c|c|c|}
\cline { 2 - 5 } \multicolumn{1}{c|}{} & GI & GII & GIII & Alle \\
\hline $1-1$ & 10 & 1 & 0 & 11 \\
\hline $1-0$ & 8 & 9 & 3 & 20 \\
\hline $0-1$ & 3 & 1 & 0 & 4 \\
\hline $0-0$ & 5 & 15 & 15 & 35 \\
\hline \multicolumn{2}{r|}{} & & $r=0.31$ \\
\hline
\end{tabular}

Tab. 51 Item-Musterergebnis A19 $\rightarrow$ A25

Diese Aufgabenkombination verhält sich ganz anders als die in diesem Abschnitt schon besprochenen. Bedingt durch die Schwierigkeit der Aufgabe A25 ist der 1-1 Anteil mit 11 Kindern sehr tief und der Anteil der 0-0 - Löser mit 35 Kindern sehr hoch. Dieses Ungleichgewicht wiederholt sich auch bei den 1-0 und 0-1 - Lösern mit 20 resp. 4 Kindern. Auch ist der Korrelationskoeffizient $r=0.35$ weit höher als bis anhin. Die Aufgabe A25 verlangt von den Kindern, wie schon beschrieben, einiges an Konzentrationsfähigkeit und Übersicht; dies sind Eigenschaften, die in ähnlicher Weise auch bei der Aufgabe A19 verlangt werden. So ist es zu erklären, dass das Verhältnis des 1-1 Anteils zum 0-1 Anteil $11 \mathrm{zu} 4$ steht und somit wohl eine kleine Abhängigkeit zwischen den beiden Aufgaben insofern feststellbar ist, als die guten A19 - Löser auch mit der Aufgabe A25 ein wenig besser zurechtkommen. 
f) $\mathrm{A} 19 \rightarrow \mathrm{A} 37$

\begin{tabular}{|c|c|c|c|c|}
\cline { 2 - 5 } \multicolumn{1}{c|}{} & GI & GII & GIII & Alle \\
\hline $1-0$ & 2 & 4 & 3 & 9 \\
\hline $0-1$ & 7 & 9 & 1 & 17 \\
\cline { 2 - 5 } & & & &
\end{tabular}

Tab. 52 Item-Musterergebnis A19 $\rightarrow$ A37

Es gibt mit 17 0-1 - Lösern fast doppelt soviele Kinder wie die 9 1-0 - Löser bei wiederum fast gleichem Verhalten der GI und GII Gruppe. Diese Tatsache und der tiefe Korrelationskoeffizient $r=0.27$ weisen darauf hin, dass die Subtraktionsaufgabe A37 völlig losgelöst von der Reihenfolgeaufgabe A19 ist.

g) $\mathrm{A} 19 \rightarrow \mathrm{A} 30$

Ganz ähnlich der Aufgabenkombination A19 $\rightarrow$ A25 verhält sich die vorliegende Kombination A19 $\rightarrow$ A30. Eine kleine Gruppe von 8 1-1 - Lösern stehen einer großen Menge von 36 0-0 - Lösern gegenüber und 23 1-0 - Löser überwiegen die 3 0-1 - Löser bei weitem. Der Korrelationskoeffizient $r=0.25$ deutet auf Unabhängigkeit der beiden Aufgaben hin. Wie die Aufgabe A25 stellt auch die Aufgabe A30 sehr hohe Ansprüche an die Zählkompetenz der Kinder (Phase IV und V) und die Beeinflussung der Aufgabe A19 hält sich in bescheidenen Grenzen.

\subsubsection{Zusammenfassung}

Es fällt auf, dass bei allen Aufgabenkombinationen ein tiefer Korrelationskoeffizient zu verzeichnen ist, also die obere Gruppe gegenüber der unteren Gruppe die betrachteten Aufgaben nicht wesentlich besser bewältigen konnte. Die zwei Leistungsgruppen GI und GII zeigten ein sehr ähnliches Lösungsverhalten, außer dass sich die Gruppe GI natürlich kompetenter verhielt. Die Gruppe GIII war wie üblich überfordert. Man kann nun auch hier daraus schließen, dass das Beherrschen von Reihenfolgenaufgaben im Allgemeinen auf Zählkompetenz keinen oder allenfalls nur einen sehr kleinen Einfluss ausübt. 
Fazit: Mit den Aufgabenkombinationen A19 $\rightarrow$ Ann wurde die Untersuchung der Beziehungen der Piaget'schen Komponenten einerseits zu Piaget'schen Komponenten selber und andererseits zu Zählkompetenzen in allen fünf Phasen nach der PrinzipienNachher-Theorie abgeschlossen. Wie im Kapitel 8.2 ab Seite 142 schon belegt, unterstreichen die vorliegenden Analysen die bescheidenen Einflüsse der Piaget'schen Komponenten auf Zählkompetenz in allen Phasen. Die These, dass vor allem die allgemeine Leistungsfähigkeit für Zählkompetenz verantwortlich zeichnet, wurde durch die vorliegenden Ergebnisse untermauert. Erst in zweiter Linie ist das Alter und erst in dritter Linie die Kompetenzen in Piaget'schen Komponenten für die Erlangung der Zählkompetenz verantwortlich. Nach unserem Kompetenzmodell (siehe Seite 65), wonach die qualitative Veränderung des Wissens vor allem auch durch Beschäftigung und aktive Auseinandersetzung mit der Umwelt sowie durch das Durchschreiten unterschiedlicher Denkwege erfolgt, ist es nahe liegend, dass sich alle Kompetenzgebiete gegenseitig beeinflussen und sich oft wohl parallel entwickeln.

\subsubsection{Aufgabengruppe A21 $\rightarrow$ Ann}

Wie beeinflusst die Kompetenz des reinen Aufsagens der Zahlwortreihe bis 20 die übrigen Facetten der Zahlbegriffsbildung? Können unsere Untersuchungsdaten belegen, dass das Zählen förderlich für komplizierterere Anforderungen im Bereich des Zählens ist? Ist das Zählen tatsächlich das im Kapitel 4 beschriebene „Schweizer Taschenmesser“ für die Lösung von vielfältigen Aufgaben im Bereich des Zählens und des Rechnens? Wie gehen leistungsstarke Kinder mit dem Werkzeug des Zählens um, wie die andern? Dies sind die Fragen, die uns im Folgenden interessieren.

38 Kinder haben die Aufgabe A21 gemeistert und befinden sich in der oberen Gruppe, die übrigen 32 Kinder bilden die untere Gruppe. In der Gruppe GI ist das Verhältnis der A21 - Löser zu den A21 - Nichtlösern 24 zu 2, in der Gruppe GII ausgeglichen 13 zu 13 und in der Gruppe GIII 1 zu 17. Bis auf die Aufgabenkombinationen A21 $\rightarrow$ A25 und A21 $\rightarrow$ A30 sind die Anteile der 1-1 - Löser und der 0-0 - Löser ausgeglichen bei etwa 25 Kindern.

a) $\mathrm{A} 21 \rightarrow \mathrm{A} 28$ 


\begin{tabular}{|c|c|c|c|c|}
\cline { 2 - 5 } \multicolumn{1}{c|}{} & GI & GII & GIII & Alle \\
\hline $1-1$ & 18 & 6 & 1 & 25 \\
\hline $1-0$ & 6 & 7 & 0 & 13 \\
\hline $0-1$ & 2 & 3 & 0 & 5 \\
\hline $0-0$ & 0 & 10 & 17 & 27 \\
\hline \multicolumn{1}{r|}{} & & $r=0.50$ \\
\cline { 2 - 4 }
\end{tabular}

Tab. 53 Item-Musterergebnis A21 $\rightarrow$ A28

In beiden Aufgaben A21 und A28 wird das Beherrschen der Zahlwortreihe bis 20 verlangt: in der Aufgabe A21 lediglich das bloße Aufsagen derselben und in der Aufgabe A28 das Auszählen von 20 konkreten Objekten. Es ist darum nur allzu verständlich, dass der Korrelationskoeffizient $r=0.50$ beträgt und nur 5 0-1 - Löser 13 1-0 - Lösern gegenüberstehen. Diese 5 0-1 - Löser zeigen nun aber auch, dass es wohl nicht zwingend notwendig ist, die Zahlwortreihe bis $20 \mathrm{zu}$ beherrschen um erfolgreich 20 konkrete Gegenstände auszuzählen (siehe Transkripte ab Seite 201).

b) A21 $\rightarrow$ A31

\begin{tabular}{|c|c|c|c|c|}
\hline & GI & GII & GIII & Alle \\
\hline $1-0$ & 9 & 4 & 0 & 13 \\
\hline $0-1$ & 2 & 4 & 2 & 8 \\
\hline & & & & $r=0.41$ \\
\hline
\end{tabular}

Tab. 54 Item-Musterergebnis A21 $\rightarrow$ A31

Als Ergänzung zur Aufgabe A28 wird bei der Aufgabe A31 das Abzählen einer bestimmten Anzahl von Objekten gefordert. Dies erfordert nebst der korrekten Zuordnung Zahlwort - Objekt auch ein erhöhtes Erinnerungsvermögen; denn bei 11 Holzwürfeln in einer Reihe ist Schluss und der Rest der Holzwürfel bleibt auf dem Haufen zurück. Trotz diesem ein wenig erhöhtem Schwierigkeitsgrad sind die Ergebnisse der Aufgabenkombination A21 $\rightarrow$ A31 durchaus mit der vorigen Kombination vergleichbar. Der erhöhte 0-1 Anteil mit 8 Kindern rührt vor allem daher, dass es ein paar wenige Kinder gibt, die die Zählaufgabe bis 20 noch nicht bewältigten, die Zahlwörter bis 11 aber kennen und damit umzugehen wissen.

c) $\mathrm{A} 21 \rightarrow \mathrm{A} 23$ 
Erstaunlicherweise liefert die Kombination A21 $\rightarrow$ A23 eine fast identische Ergebnistabelle wie die soeben besprochene, obwohl die Aufgabe A23 eine ganz andere Kompetenz von den Kindern verlangt. Diese Beobachtung zeigt, dass Kinder, die über eine solide Zahlwortreihe verfügen, auf dieser Basis auch Aufgaben aus einer höheren Phase erfolgreich bewältigen. Der Korrelationskoeffizient $r=0.41$ sowie die 1-0 und 0-1 Anteile sind mit der vorigen Kombination fast identisch und zeigen so auch hier die Wirkung der Grundlagenkompetenz der Aufgabe A21.

d) $\mathrm{A} 21 \rightarrow \mathrm{A} 25$

\begin{tabular}{|c|c|c|c|c|}
\cline { 2 - 5 } \multicolumn{1}{c|}{} & GI & GII & GIII & Alle \\
\hline $1-1$ & 12 & 2 & 0 & 14 \\
\hline $1-0$ & 12 & 11 & 1 & 24 \\
\hline $0-1$ & 1 & 0 & 0 & 1 \\
\hline $0-0$ & 1 & 13 & 17 & 31 \\
\hline \multicolumn{3}{r|}{} & $r=0.41$ \\
\cline { 2 - 4 }
\end{tabular}

Tab. 55 Item-Musterergebnis A21 $\rightarrow$ A25

Ohne Kenntnis der Zahlwortreihe läuft bei der Aufgabe A25 offensichtlich nichts. Vor allem die Gruppe GII und auch das Gesamtergebnis zeigt im Vergleich der 1-1 - Löser und der 1-0 - Löser aber auch, dass die Beherrschung des Zählens allein nicht reicht, um diese schwere Aufgabe A25 zu lösen; viele Kinder haben die Phase IV der Auffassung der Zahlwörter als zählbare Einheiten noch nicht erreicht oder ihre Kompetenzen sind in dieser Phase noch nicht gefestigt.

e) A21 $\rightarrow$ A37

\begin{tabular}{|c|c|c|c|c|}
\hline & GI & GII & GIII & Alle \\
\hline 1-1 & 22 & 7 & 0 & 29 \\
\hline $1-0$ & 2 & 6 & 1 & 9 \\
\hline 0-1 & 1 & 8 & 1 & 10 \\
\hline $0-0$ & 1 & 5 & 16 & 22 \\
\hline
\end{tabular}

Tab. 56 Item-Musterergebnis A21 $\rightarrow$ A37

Schlichtes Zählen, zählendes Rechnen oder der äußere Eindruck können bei der Aufgabe A37 zum richtigen Ergebnis führen. Der hohe Korrelationskoeffizient $r=$ 0.45 lässt darauf schließen, dass die guten Zähler gegenüber den Nichtzählern bei dieser Subtraktionsaufgabe im Vorteil sind. Die Gruppe GII zeigt aber ein ganz anderes Bild: hier ist es für ein richtiges Ergebnis ganz egal, ob man zählen kann oder 
nicht $\left(r_{\mathrm{GII}} \approx 0.00\right)$. Von den 26 Kindern der Gruppe GI lösen hingegen 23 die Aufgabe A37, davon ist lediglich ein Kind Nichtzähler. Die allermeisten leistungsfähigen Kinder können zählen und mit dieser Kompetenz und mit diesem Werkzeug sind sie in der Lage, auch Operationen durchzuführen.

f) $\mathrm{A} 21 \rightarrow \mathrm{A} 30$

Die Ergebnistabelle der vorliegenden Aufgabenkombination A21 $\rightarrow$ A30 ist der Ergebnistabelle A21 $\rightarrow$ A25 sehr ähnlich (siehe Seite 272). Es ist selbstverständlich, dass hier ohne Kenntnis der Zahlwortreihe das Rückwärtszählen nicht möglich ist. (Der einzige 0-1 - Löser war bei der Aufgabe A21 sehr unkonzentriert und hat darum beim Aufsagen der Zahlwortreihe bis 20 versagt.) Die letzte Phase V der Prinzipien-Nachher-Theorie der „zweiseitigen Durchlaufbarkeit“ der Zahlwortreihe haben die meisten Kinder noch nicht erreicht.

\subsubsection{Zusammenfassung}

Im Gegensatz zu den Beziehungen Piaget'sche Komponenten - Zählkompetenz sind die Korrelationskoeffizienten bei allen in diesem Abschnitt vorliegenden Aufgabenkombinationen relativ hoch. Dies heißt, dass die obere Gruppe durchaus von ihren Zählkompetenzen bei der Lösung der übrigen Aufgaben profitieren konnte. Das Lösungsverhalten der beiden Gruppen GI und GII ist in der Tendenz meistens vergleichbar, außer bei den schwierigen Aufgabenkombinationen A21 $\rightarrow$ A25 und A21 $\rightarrow$ A30. Bei diesen Kombinationen spielt wohl nicht die Zählkompetenz die Hauptrolle, sondern eher das allgemeine Leistungsniveau. Wie immer ist die Gruppe GIII überfordert. Wesentlich ist die Beobachtung, dass das Beherrschen der Zahlwortreihe bis 20 vorteilhaft ist für das Bewältigen von komplizierteren Problemen und Aufgabenstellungen.

Fazit: Das Beherrschen der Zahlwortreihe eröffnet den Kindern die Möglichkeit, in die übrigen Phasen der Zählkompetenz vorzustoßen und stellt tatsächlich ein fundamentales Werkzeug dar. Es wird eine noch weiterführende Untersuchung nötig sein, um die These zu erhärten, dass solides Zählvermögen im ersten Mathematikunterricht von großem Vorteil ist. 


\subsubsection{Aufgabengruppe A28 $\rightarrow$ Ann}

Wie im vorherigen Kapitel interessiert uns hier die Einflussnahme der Auszählaufgabe A28 auf die Aufgaben der übrigen Phasen. Wie beeinflusst die Kompetenz des Auszählens von 20 Objekten das Lösungsverhalten bei anderen Aufgaben? Können diese zusätzlichen Ergebnisse belegen, dass das konkrete Zählen förderlich ist für kompliziertere Anforderungen im Bereich des Zählens?

30 Kinder haben die Aufgabe A28 gemeistert und befinden sich in der oberen Gruppe, die übrigen 40 Kinder bilden die untere Gruppe. In der Gruppe GI ist das Verhältnis der A28 - Löser zu den A28 - Nichtlösern 20 zu 6, in der Gruppe GII 9 zu 17 und in der Gruppe GIII 1 zu 17. Wieder bis auf die Aufgabenkombinationen A28 $\rightarrow$ A25 und A28 $\rightarrow$ A30 sind die Anteile der 1-1 - Löser und der 0-0 - Löser eher ausgeglichen bei etwa 20 zu 28 Kindern.

a) $\mathrm{A} 28 \rightarrow \mathrm{A} 31$

\begin{tabular}{|c|c|c|c|c|}
\cline { 2 - 5 } \multicolumn{1}{c|}{} & GI & GII & GIII & Alle \\
\hline $1-1$ & 14 & 5 & 1 & 20 \\
\hline $1-0$ & 6 & 4 & 0 & 10 \\
\hline $0-1$ & 3 & 7 & 2 & 12 \\
\hline $0-0$ & 3 & 10 & 15 & 28 \\
\hline
\end{tabular}

Tab. 57 Item-Musterergebnis A28 $\rightarrow$ A31

Da Kinder in der Phase II das Auszählen (Aufgabe A28) wie auch das Abzählen (Aufgabe A31) beherrschen, ist das Ergebnis dieser Kombination dahingehend überraschend, als man erwarten würde, dass die 1-0 und die 0-1 Anteile tief sind. Vor allem die relativ hohen 1-0 und 0-1 Ergebnisse lassen doch darauf schießen, dass es für die Lösung der Aufgabe A31 eigentlich völlig egal ist, ob man die Aufgabe A28 erfolgreich bewältigt hat und umgekehrt. Wie schon einmal erwähnt, könnte der relativ hohe 0-1 Anteil daher rühren, dass A31 nur eine Kollektion von 11 Objekten erfordert und A28 mit 20 Objekten operiert und einige Kinder mit 11 Gegenständen umgehen können, 20 Gegenstände aber noch nicht schaffen. Andererseits verlangt die Aufgabe A28 eigentlich nur eine Bestandesaufnahme, wohingegen A31 eine aktive Entscheidung bezüglich des Aufhörens (nur 11 Holzwürfel hinlegen von einem 
Haufen von 15 Würfeln) erfordert. Die obere Gruppe ist mit dem Korrelationskoeffizient $r=0.36$ leicht besser in der Herstellung der geforderten Reihe.

b) $\mathrm{A} 28 \rightarrow \mathrm{A} 23$

Die eben besprochene Ergebnistabelle wiederholt sich in beinahe identischer Form für die Aufgabenkombination A28 $\rightarrow$ A23 (siehe Seite 272). Dass hier die Aufgabe A28 wenig Einfluss auf das Lösen der Aufgabe A23 ausübt, verwundert hingegen weniger, da die Aufgabe A23 mit dem Weiterzählen-Können doch andere Kompetenzen abfragt als die Aufgabe A31. Den 9 1-0 - Lösern stehen 12 0-1 - Löser gegenüber, was den geringen Einfluss der Aufgabe A28 untermauert. Der Korrelationskoeffizient $r=0.40$ zeigt immerhin die bessere Behandlung der Aufgabe A23 durch die oberen Gruppe.

c) $\mathrm{A} 28 \rightarrow \mathrm{A} 25$

\begin{tabular}{|l|l|l|l|l|}
\cline { 2 - 5 } \multicolumn{1}{c|}{} & GI & GII & GIII & Alle \\
\hline $1-1$ & 11 & 1 & 0 & 12 \\
\hline $1-0$ & 9 & 8 & 1 & 18 \\
\hline $0-1$ & 2 & 1 & 0 & 3 \\
\hline $0-0$ & 4 & 16 & 17 & 37 \\
\hline
\end{tabular}

Tab. 58 Item-Musterergebnis A28 $\rightarrow$ A25

Die Kinder sind bei dieser Kombination wegen der Aufgabe A25 wieder überfordert. Von den 30 A28 Lösern schaffen nur gerade 12 die Aufgabe A25 und ganze drei von 40 Kindern, die die Aufgabe A28 nicht lösen, sind bei der Aufgabe A25 erfolgreich. Immerhin ist die obere Gruppe mit dem Korrelationskoeffizient $r=0.39$ für Aufgaben des Typs A25 besser gerüstet.

d) $\mathrm{A} 28 \rightarrow \mathrm{A} 37$

Die Ergebnisse dieser Kombination lassen sich mit den Ergebnissen der Kombinationen A28 $\rightarrow$ A31 und A28 $\rightarrow$ A23 durchaus vergleichen. 8 1-0 - Löser und 17 0-1 - Löser zeigen, dass die Einflussnahme der Aufgabe A28 auf diese Subtraktionsaufgabe doch sehr gering ist. Das Auszählen von Objekten zeigt wohl nur geringe Wir- 
kung auf die Möglichkeit, neue Situationen zu erkennen und zu bearbeiten. Der Korrelationskoeffizient $r=0.31$ ist denn auch tiefer als die vorhergehenden.

e) $\mathrm{A} 28 \rightarrow \mathrm{A} 30$

Bis auf den Korrelationskoeffizient $r=0.18$ gegenüber $r=0.39$ ist die Ergebnistabelle wiederum sehr ähnlich der Tabelle der Aufgabenkombination A28 $\rightarrow$ A25 (siehe oben). Wohl kaum besser gewappnet für das Rückwärtszählen ist die obere Gruppe. Nur gerade 7 Kinder von 30 dieser oberen Gruppe schaffen die Aufgabe, aber auch 4 Kinder der unteren Gruppe.

\subsubsection{Zusammenfassung}

Wie bei den Aufgabenkombinationen A21 $\rightarrow$ Ann sind auch bei den vorliegenden Kombina-tionen die Korrelationskoeffizient relativ hoch, was besagt, dass die guten A28 - Löser auch die übrigen Aufgaben besser bewältigen als diejenigen Kinder, welche die Aufgabe A28 nicht gelöst haben. Die bis auf die Kombinationen mit den Aufgabe A25 und A30 doch ausgeglichenen 1-0 und 0-1 Anteile weisen aber doch darauf hin, dass die Auszählkompetenz nicht ausreicht, um Aufgaben aus den folgenden Phasen sicher zu bewältigen. Auch hier ist die Gruppe GIII überfordert und die Gruppen GI und GII sind - was die 1-0 und 0-1 Ausfälle ausmacht - vergleichbar, was bedeutet, dass es kein prinzipiell anderes Lösungsverhalten dieser beiden Gruppen gibt.

\subsubsection{Aufgabengruppe A23 $\rightarrow$ Ann}

Die Kinder, welche die folgenden Aufgaben lösen können, befinden sich in einem schon fortgeschrittenen Stadium der Zählkompetenz.

33 Kinder haben die Aufgabe A23 gelöst und befinden sich in der oberen Gruppe, die übrigen 37 Kinder bilden die untere Gruppe. In der Gruppe GI ist das Verhältnis der A23 - Löser zu den A23 - Nichtlösern 19 zu 7, in der Gruppe GII 13 zu 13 und in der Gruppe GIII 1 zu 17. 
a) $\mathrm{A} 23 \rightarrow \mathrm{A} 25$

\begin{tabular}{|c|c|c|c|c|}
\hline & GI & GII & GIII & Alle \\
\hline $1-1$ & 9 & 0 & 0 & 9 \\
\hline $1-0$ & 10 & 13 & 1 & 24 \\
\hline $0-1$ & 4 & 2 & 0 & 6 \\
\hline $0-0$ & 3 & 11 & 17 & 31 \\
\hline
\end{tabular}

Tab. 59 Item-Musterergebnis A23 $\rightarrow$ A25

Offenbar ist der Einfluss der Aufgabe A23 auf die Aufgabe A25 gering; es gibt 9 11 - Löser, aber auch 6 0-1 - Löser. Der Korrelationskoeffizient $r=0.13$ deutet dann auch darauf hin, dass die obere Gruppe gegenüber der unteren Gruppe nicht besser mit der Aufgabe A25 fertig wird. Auf den ersten Blick ist dieser Befund eigentlich erstaunlich, da die beiden Aufgaben eine ganz ähnliche Struktur aufweisen: weiterzählen von einer bestimmten Zahl ungleich Eins. Der fundamentale Unterschied, der die Schwierigkeit der Aufgabe A25 erklärt, ist das verlangte schrittweise Vorwärtsschreiten mit einer Schrittweite ungleich Eins. Die Zahlwörter sind in dieser Phase selber als zählbare Einheiten aufzufassen, was von den meisten Kindern offenbar noch nicht ganz verstanden wird. Der hohe 1-0 Anteil ist wegen der Schwierigkeit der Aufgaben nicht verwunderlich.

b) $\mathrm{A} 23 \rightarrow \mathrm{A} 37$

\begin{tabular}{|c|c|c|c|c|}
\cline { 2 - 5 } \multicolumn{1}{c|}{} & GI & GII & GIII & Alle \\
\hline $1-1$ & 16 & 8 & 0 & 24 \\
\hline $1-0$ & 3 & 5 & 1 & 9 \\
\hline $0-1$ & 7 & 7 & 1 & 15 \\
\hline $0-0$ & 0 & 6 & 16 & 22 \\
\hline \multicolumn{1}{r}{} & $r=0.36$ \\
\cline { 2 - 4 } & &
\end{tabular}

Tab. 60 Item-Musterergebnis A23 $\rightarrow$ A37

Hier ist der Einfluss der Aufgabe A23 auf die Lösbarkeit der Aufgabe A37 wohl größer. Auch der Korrelationskoeffizient $r=0.36$ zeigt, dass die obere Gruppe mit der Aufgabe A37 besser zurande kommt. Es fällt aber auf, dass es einen sehr hohen 0-1 Anteil mit 15 Kindern gibt, was den Schluss zulässt, dass die Subtraktionsaufgabe auch ohne große Zählkompetenz durchaus lösbar ist, da ja noch andere Lösungswege zur Verfügung stehen. 
c) $\mathrm{A} 23 \rightarrow \mathrm{A} 30$

\begin{tabular}{|c|c|c|c|c|}
\cline { 2 - 5 } \multicolumn{1}{c|}{} & GI & GII & GIII & Alle \\
\hline $1-0$ & 9 & 13 & 1 & 23 \\
\hline $0-1$ & 0 & 1 & 0 & 1 \\
\hline \multicolumn{4}{r}{}
\end{tabular}

Tab. 61 Item-Musterergebnis A23 $\rightarrow$ A30

Hier stehen 23 1-0 Kinder nur einem einzigen 0-1 Kind gegenüber, was wiederum bestätigt, dass die Aufgabe A30 schwer ist. Die 10 1-1 - Löser haben die Aufgabe wohl nicht darum gelöst, weil sie die Aufgabe A23 erfolgreich bewältigten, sondern wohl eher auf Grund ihrer allgemein hohen Leistungsfähigkeit.

\subsubsection{Zusammenfassung}

Die schweren Aufgaben A25 und A30 können von erfolgreichen A23 - Lösern zwar markant besser gelöst werden als von den übrigen Kindern. Die 1-0 Anteile dieser beiden Aufgaben sind aber mehr als doppelt so hoch wie die 1-1 Anteile, was darauf hinweist, dass die Aufgabe A23 wohl keine große Unterstützung bei der Lösung der Aufgaben A25 und A30 bietet. Wie die 1-0 und die 0-1 Anteile belegen, verhalten sich die beiden Gruppen GI und GII in ihrem Lösungsverhalten durchwegs ähnlich.

\subsubsection{Aufgabengruppe A25 $\rightarrow$ Ann}

15 Kinder haben die Aufgabe A25 gelöst und befinden sich in der oberen Gruppe, die untere Gruppe besteht aus den übrigen 55 Kindern. In der Gruppe GI ist das Verhältnis der A25 - Löser zu den A25 - Nichtlöser 13 zu 13, in der Gruppe GII 2 zu 24 und in der Gruppe GIII 0 zu 18.

a) $\mathrm{A} 25 \rightarrow \mathrm{A} 37$

\begin{tabular}{|c|c|c|c|c|}
\cline { 2 - 5 } \multicolumn{1}{c|}{} & GI & GII & GIII & Alle \\
\hline $1-0$ & 1 & 0 & 0 & 1 \\
\hline $0-1$ & 11 & 13 & 1 & 25 \\
\hline \multicolumn{4}{r|}{} & $r=0.40$ \\
\hline
\end{tabular}


Tab. 62 Item-Musterergebnis A25 $\rightarrow$ A37

Es ist nicht verwunderlich, dass es bei dieser Kombination „,schwer" $\rightarrow$ „leicht“" nur einen 1-0 Ausfall gegenüber 25 0-1 Ausfällen gibt. Die obere Gruppe ist aber doch deutlich gewandter bei der Lösung der Aufgabe A37: von den 15 Kindern, welche die Aufgabe A25 lösten, haben immerhin 14 auch die Aufgabe A37 gemeistert.

b) $\mathrm{A} 25 \rightarrow \mathrm{A} 30$

\begin{tabular}{|c|c|c|c|c|}
\cline { 2 - 5 } \multicolumn{1}{c|}{} & GI & GII & GIII & Alle \\
\hline $1-1$ & 7 & 0 & 0 & 7 \\
\hline $1-0$ & 6 & 2 & 0 & 8 \\
\hline $0-1$ & 3 & 1 & 0 & 4 \\
\hline $0-0$ & 10 & 23 & 18 & 51 \\
\hline \multicolumn{4}{r|}{} & $r=0.44$ \\
\cline { 2 - 5 }
\end{tabular}

Tab. 63 Item-Musterergebnis A25 $\rightarrow$ A30

Natürlich ist der Korrelationskoeffizient mit $r=0.44$ hoch, da bei dieser wohl schwersten Aufgabenkombination die meisten Kinder zur 0-0 Gruppe gehören und knapp die Hälfte der 15 A25 - Löser auch die Aufgabe A30 gelöst haben. Obwohl der Einfluss der Aufgabe A25 auf die Lösungskompetenz der Aufgabe A30 als nicht allzu groß einzustufen ist, bleibt doch zu bedenken, dass bei beiden Aufgaben die Zahlwortreihe mit großer Virtuosität beherrscht werden muss. Ist die Kompetenz für die Lösung der Aufgabe A25 vorhanden, ist der Weg zur gesicherten beidseitiger Durchlaufbarkeit der Zahlwortreihe nicht mehr weit.

\subsubsection{Zusammenfassung}

Bei beiden Kombinationen ist die obere Gruppe weit erfolgreicher als die untere Gruppe, obwohl die 1-0 und 0-1 Anteile sich sehr voneinander unterscheiden (1 zu 25, resp. $8 \mathrm{zu}$ 4), was bedeutet, dass keinerlei Beeinflussung der Aufgabe A25 auf das Lösen der Aufgabe A37 und wenig Einfluss auf die Aufgabe A30 stattfindet. Auch gibt es keinen Unterschied im Lösungsverhalten der beiden Gruppen GI und GII; die Proportionen der 1-0 und 0-1 Anteile sind fast identisch. 


\subsubsection{Aufgabengruppe A37 $\rightarrow$ A30}

\begin{tabular}{|c|c|c|c|c|}
\cline { 2 - 5 } \multicolumn{1}{c|}{} & GI & GII & GIII & Alle \\
\hline $1-0$ & 15 & 15 & 1 & 31 \\
\hline $0-1$ & 2 & 1 & 0 & 3 \\
\hline \multicolumn{4}{r|}{}
\end{tabular}

Tab. 64 Item-Musterergebnis A37 $\rightarrow$ A30

39 Kinder haben die Aufgabe A37 gelöst und befinden sich in der oberen Gruppe, die übrigen 31 Kinder stellen die untere Gruppe. In der Gruppe GI ist das Verhältnis der A37 - Löser zu den A37 - Nichtlösern 23 zu 3, in der Gruppe GII 15 zu 11 und in der Gruppe GIII 1 zu 17.

Das Verhältnis der 1-0 zu den 0-1 - Lösern ist mit $31 \mathrm{zu} 3$ beinahe umgekehrt zur Kombination der Aufgaben A25 $\rightarrow$ A37; hier handelt es sich um eine Beziehung „leicht“ $\rightarrow$ „schwer“. Nur 8 Kinder von den 39 A37 - Lösern bewältigten die Aufgabe A30 und immerhin 3 Kinder können rückwärts zählen, ohne imstande zu sein, die leichte Subtraktionsaufgabe mit Erfolg zu lösen. Der Korrelationskoeffizient $r=$ 0.15 unterstreicht die Beobachtung, dass die obere Gruppe nicht wesentlich besser abschneidet als die untere Gruppe.

\subsubsection{Zusammenfassung}

Der Vergleich der Beziehungen von pränumerischen Aufgaben im Sinne von Piaget untereinander und dann insbesondere mit numerischen Problemen im Sinne von Zählaufgaben legt offen, dass die Bedeutung der pränumerischen Aktivitäten auf die Zählkompetenz einen relativ kleinen Einfluss ausübt. Bemerkenswert scheint mir ebenfalls die schwache Wechselwirkung der Piaget'schen Aufgaben untereinander, wie die Korrelationskoeffiziententabelle im Anhang auf der Seite 278 dokumentiert.

Selbstverständlich ist eine gute Eins-zu-eins-Zuordnungskompetenz oder eine fundierte Seriationskompetenz für die Zählentwicklung nicht abkömmlich, aber die Beeinflussung ist nie im Sinne von Ausschließlichkeit zu verstehen, sondern allenfalls als unterstützendes Element. Kinder, die Gegenstände abzählen, vollführen oft in selbstverständlicher 
Art und Weise eine Eins-zu-eins-Zuordnung Gegenstand - Zahlwort: oft sogar, indem sie mit dem Finger auf das zu zählende Objekt zeigen oder es gar berühren und in diesem Moment das Zahlwort aufsagen (siehe nächstes Kapitel 9). Eine Reihenfolge wird bei ordinalen Zählsituationen als Ordnung in Form einer Reihe oft automatisch hergestellt. Solche und viele andere Situationen zeigen, dass die verschiedenen Kompetenzen im pränumerischen und numerischen Bereich sich gegenseitig unterstützen und sich vielfach zeitlich parallel entwickeln und entfalten. Diese Einsicht sollte bei der Konzeption von mathematischer Frühförderung im Kindergarten einfließen. Im Kapitel 10.2 werden solche Konzepte vorgestellt.

Es hat sich gezeigt, dass das reine Aufsagen der Zahlwortreihe sehr förderlich ist für anspruchsvollere, komplizierte Anforderungen im numerischen Bereich. Die Funktion des Zählens als „Schweizer Taschenmessers“ im Sinne von vielfältiger Einsetzbarkeit ist sehr wohl gegeben. Leistungsstarke Kinder besitzen eine höhere Performanz des Zählens und sind dadurch weit besser in der Lage, dieses Werkzeug gezielt einzusetzen.

Wer den Zählvorgang versteht, kann alles Mögliche über Zahlen herausfinden. Durch das Zählen werden in natürlicher Weise viele fundamentale pränumerische Kompetenzen wie die hier in diesem Kapitel oft besprochenen erst aktiviert, ohne allzu künstliche Situationen im Sinne der noch weit verbreiteten „Mengenlehre“ zu bemühen. Im Kapitel 10.2 gebe ich konkrete Beispiele für die Förderung der allgemeinen Zählkompetenz. Mit dem Effekt, dass viele andere mathematische Gebiete in ganz natürlicher Art und Weise von den Kindern entdeckt, zur Kenntnis genommen und mit ihren eigenen Möglichkeiten weiterentwickelt werden. Die Entwicklung der Zahlerfassung im Sinne von Anzahlerfassung durch Zählen, aber auch durch simultane Anzahlerfassung, dem Erkennen von Zahlbildern oder Muster sollte bei der vorschulischen Förderung, auf die ich im Kapitel 10.2 noch zurückkomme, als Kondensationskern betrachtet werden. Als Forderung mit dem Ziel einer optimalen mathematischen Förderung kann aber festgehalten werden, dass die Kindern so früh wie möglich zum Zählen angeleitet und dabei unterstützt werden sollten, da eine fundierte Zählkompetenz insbesondere direkt vor und beim Schulbeginn zentral ist. Beim Addieren, aber auch beim Subtrahieren in einem überblickbaren Zahlenraum können Kinder im Wesentlichen die folgenden - auf Zählen aufbauende Lösungsstrategien anwenden ${ }^{50}$. Als erstes sei das vollständige Auszählen erwähnt. Bei dieser Strategie werden beim Addieren beide Anzahlen mit Gegenständen, Strichen oder

\footnotetext{
${ }^{50}$ Ich halte mich im Folgenden vor allem an Gerster (1994) „Arithmetik im Anfangsunterricht“.
} 
mit den Fingern (falls diese ausreichen) ${ }^{51}$ dargestellt und danach abgezählt. Beim Subtrahieren wird zuerst der Minuend auf die gleiche Art wie eben beschrieben behandelt. Dann wird innerhalb dieser Menge der Subtrahend ausgezählt und diese Teilmenge weggenommen. Zum Schluss wird der verbleibende Rest ausgezählt. Bei der zweiten Strategie - dem Weiterzählen - wird von einem der beiden Summanden vorwärts, beziehungsweise vom Minuenden rückwärts oder vom Subtrahenden (Ergänzen) vorwärts gezählt. Oft wird beim Addieren das Kommutativgesetz angewendet in der Art, dass die Kinder vom größeren Summanden aus weiterzählen. Das Weiterzählen (z.B. bei $4+3$ ) geschieht einerseits mit den Findern, resp. mit Material: 4 Finger zeigen, dann 3 Finger dazutun und dabei gleich sprechen ,5, 6, 7“ oder ohne die 4 darzustellen gleich mit Hilfe der 3 Fingern ,5, 6, 7“ weiterzählen. Andererseits wird auch ohne Material die Technik des Weiterzählens angewendet in der Art, dass gleich von der 4 weitergezählt wird und auch durch doppeltes Zählen kontrollieret wird, z.B. ,plus 1 gibt 5, plus 2 gibt 6, plus 3 gibt 7“. Das zählende Rechnen hat bei Aufgaben des kleinen Einspluseins und beim Einmaleins folgende Vorzüge:

Diese Technik wird von den Kindern spontan oder auch gelenkt schon im Kindergarten gelernt.

- Die Technik des zählenden Rechnens garantiert durch die Einheitlichkeit der Strategie und durch die ,auf der Hand liegenden“ Begründbarkeit eine (scheinbare) Sicherheit im Umgang mit arithmetischen Grundaufgaben.

Für den Erwerb erster arithmetischer Kompetenzen ist das zählende Rechnen fundamental und kann durchaus ein wichtiges Glied darstellen in der Entwicklung von Einsichten in zahlreichen Zahlbeziehungen.

Die Gefahren des zählenden Rechnens dürfen hier natürlich nicht verschwiegen werden und es scheint mir wichtig, dass sich die Lehrkräfte der folgenden aufgeführten Probleme des zählenden Rechnens bewusst sind. Wird die mathematische Tätigkeit nur auf das Zählen eingeschränkt, würde das allzu oft in die Sackgasse der Rechenschwäche führen (vgl. Gerster 1994, 45).

Die Strategien des vollständigen Auszählens bei Addition und Subtraktion sind umständlich, die Weiterzählstrategien verlangen doppeltes Zählen (z.B. bei der

\footnotetext{
${ }^{51}$ Anfangs finden Kinder es schwierig, ohne Benutzung der Finger zu rechnen. Wörter verschwinden, kaum sind sie ausgesprochen. Finger können aber im Blickfeld bleiben, was verhindert, dass man den Faden verliert, wenn man mal abgelenkt wird (vgl. Dehaene 1999, 143).
} 
Subtraktion 7 - 3: 7 minus 1 sind 6, minus 2 sind 5, minus 3 sind 4) oder die Kinder benutzen ihre Finger.

Da die Rolle des Anfangs- oder Endgliedes nicht immer klar definiert wird, ist das Ergebnis um \pm 1 daneben.

Sogenannte Zählkinder verwenden nicht die bereits gelernten Zahlensätze, sondern sie tendieren zur stereotypen Anwendung ihrer Zähltechniken, die dann bei größeren Zahlen immer umständlicher und dann gar nicht mehr anwendbar sind. Zähltechniken können trainiert und perfektioniert werden. Dabei schwindet das Bedürfnis und (am Anfang) die Notwendigkeit, sich Zahlensätze zu merken.

Zählkinder benutzen Beziehungen zwischen Zahlensätzen nicht. Zusammenhänge werden nicht zur Kenntnis genommen. So lösen Zählkinder die Aufgabe $3+3$ zählend und tun dasselbe anschließend mit $3+4$, ohne den Zusammenhang zwischen den beiden Aufgaben zur Kenntnis zu nehmen oder die beiden nacheinander gestellten Aufgaben $3+4$ und $13+4$ berechnen sie jeweils zählend, ohne sich der dekadischen Analogie bewusst zu werden.

Wenn Kinder im Laufe der Schuljahre Fakten immer noch nicht auswendig wissen, verzichten sie auf Merkversuche ganz und verlassen sich auf instrumentelle Nutzung von Materialien, vor allem der Finger.

Die Aufmerksamkeit von Zählkindern richtet sich vor allem auf die Zählprozedur und weniger (oder überhaupt nicht) auf den Zusammenhang zwischen Aufgabe und Ergebnis.

Aus diesem Grunde liefert das zählende Rechnen jeweils nur Einzelfakten. Diese sind nicht in ein numerisch/arithmetisches Beziehungsgeflecht eingebettet und gehen darum leicht in Vergessenheit.

Bei anspruchsvolleren Aufgaben aus der Arithmetik, beim Lösen von numerischen Aufgaben aus dem Gebiet des Sachrechnens oder bei Berechnungen in der Geometrie beansprucht das zählende Rechnen immer viel Aufmerksamkeit, die dann für die Planung von Lösungsstrategien und das Einhalten von Verfahrensregeln nicht mehr zur Verfügung steht.

Wie auf der Seite 73 beschrieben, sind einmal als nützlich ausgewiesene mentale Modelle schwer zu ändern. Alte Verhaltenstrukturen treten vor allem bei Stresssituationen (z.B. bei Klassenarbeiten, Wettrechnen, u.a.) wieder auf. So sind Rückfälle zum zählenden Rechnen gerade in solchen Situationen sehr häufig anzutreffen. Wenn sich ein Kind zum permanenten „Zähler“ entwickelt hat, sind 
Hilfsmassnahmen dringend notwendig und es ist sehr schwer, solche Kinder zum denkenden Rechnen anzuleiten, „weil die betreffenden Schüler über Jahre eine bestimmte Vorstellung vom Zahlenraum, von den Zahlbeziehungen und den Rechenoperationen verfestigt haben“ (Lorenz \& Radatz 1993, 117 und insbesondere Gerster 1994, 43-46).

Diese Ausführungen widersprechen sich in keiner Weise zu der zu Anfang dieses Kapitels ab Seite 185 geäußerten Ansicht, dass das Zählen zu Beginn der arithmetischen Gehversuche ein vorzügliches Werkzeug darstellt zur Bewältigung vielerlei numerischer Probleme. Es ist aber von fundamentaler Wichtigkeit, die Technik des zählenden Rechnens abzulösen mit Strategien des denkenden Rechnens. 


\section{Kompetenzentwicklung und -unterschiede einzel- ner Kinder}

In diesem Kapitel werde ich meine Beobachtungen des Lösungsverhaltens der einzelnen Kinder festhalten und miteinander vergleichen. So lassen sich individuelle Unterschiede ausmachen, das Verhalten der Erfolgreichen dem Verhalten der weniger Erfolgreichen gegenüberstellen und Tendenzen zu Erfolg versprechendem Umgang mit numerischen Aufgaben und Problemen festhalten. Ein mögliches Ziel ist es, Empfehlungen zu einem Erfolg verheißenden Einstieg in die Vorschulmathematik und schlussendlich in die Grundschulmathematik zu formulieren. Bei dieser Sichtung konzentriere ich mich vor allem auf die numerischen Komponenten, das heißt auf die Aufgaben A21 bis A40, ohne die übrigen Aufgaben ganz zu vernachlässigen.

\subsection{Vergleichskonzepte}

Es gibt in dieser ersten Komponente „Vergleichskonzepte“ zwei Aufgaben, die man auch mit Zählen lösen könnte (Die Aufgaben A4 und A5, siehe ab Seite 107). Von allen 70 Kindern haben gesamthaft aber nur jeweils drei Kinder die Aufgaben mit Auszählen gemeistert, alle andern versuchten mit großem Erfolg die Aufgaben nur über den visuellen Eindruck zu lösen. Die Kinder zeigten vielfach ohne zu zögern und mit großem Selbstbewusstsein auf das richtige Bild. Die simultane Anzahlerfassung fällt hier weg, da die allermeisten Kinder die beiden Aufgabenblätter als Gesamtes schnell überblickten, die einzelnen Bilder kaum beachteten, um dann sofort - und das ist entscheidend mit den Fingern auf das richtige Bild zu tippen. Die Anzahl der einzelnen Objekte (Federn, Murmeln) spielte für die Kinder bei dieser Art von Lösungsstrategie überhaupt keine Rolle; allein der für die Gegenstände benötigte Platz war für die Entscheidung maßgebend. Aus dieser Sicht scheint es verständlich, dass Erfolg oder Misserfolg in den Vergleichkonzeptaufgaben keinen Einfluss auf die Zählkompetenzen haben. Bei diesem Lösungsverhalten zeigten sich keine Unterschiede bei den Kindern mit hohem Gesamtresultat gegenüber den Kindern mit tieferem Gesamtresultat. 
Es ist im Übrigen in keiner Leistungs- resp. Altersgruppe ein weiteres auffälliges oder bemerkenswertes Lösungsverhalten bei diesen fünf Fragen der Vergleichskomponente auszumachen.

\subsection{Klassifizierung}

Auch in dieser Komponente wäre es möglich, eine Aufgabe mit Zählen zu lösen ${ }^{52}$. Wie in der vorigen Komponente haben auch hier nur drei Kinder von dieser Möglichkeit Gebrauch gemacht. In diesem Fall ist das natürlich nur allzuverständlich, da die Aufgabe so formuliert ist, dass die Kinder ohne Zählkompetenz auch auf das richtige Resultat kommen können. ${ }^{53}$

Ganz junge Kinder (Alterstufe I und II) haben oft noch Schwierigkeiten, geometrische Objekte wie zum Beispiel Quadrate, Rechtecke, Dreiecke und Kreise als solche überhaupt zu erkennen und/oder voneinander zu unterscheiden; ${ }^{54}$ und genau diese Kompetenz wird von den Kindern in zwei Aufgaben gefordert. „Kreis, was ist das?“ oder „Was meinst du?“, ... Solche und ähnliche Rückfragen kamen ausschließlich bei Kindern der Alterstufe I und II vor. Aus dieser Sicht liegt es natürlich auf der Hand, dass die jüngeren Kinder bei der Lösung dieser Aufgaben benachteiligt sind. Es ist durch verschiedene Studien belegt (Piaget/Inhelder 1973; Kofsky 1963, 1966; zur Oeveste 1987), dass einfache Klassifikation der multiplen Klassifikation vorausgeht. Zur Oeveste modifiziert die durch Piaget/Inhelder vorgegebene zeitliche Abfolge, indem er den Zeitpunkt der einfachen Klassifikation von 5;6 Jahre auf 2 Jahre und die multiple Klassifikation von 7;6 auf 6 Jahre vorverlegt (zur Oeveste 1987, 32). Genau dieses letzte Ergebnis ist durch die vorliegende Untersuchung untermauert worden: von den 31 Kindern, die den Alterstufen I, II und III angehören (unter 6 Jahre alt), haben 18 die Aufgabe A9 und 14 die Aufgabe A10 gelöst.

Die Art der Lösungsschemata unterscheidet sich nicht zwischen den Kindern mit hohem Gesamtergebnis und den Kindern mit tiefem Gesamtergebnis.

\footnotetext{
${ }^{52}$ Es handelt sich um die Aufgabe A7 (siehe Seite 109)

${ }^{53}$ Es sei daran erinnert, dass nur ein Bild ohne Dreiecke vorkommt, und dieses Bild hat genau fünf Quadrate.

${ }^{54}$ Erst ab ca. vier Jahren entwickelt sich eine fortschreitende Differenzierung der euklidischen Formen resp. der geometrischen Objekte (Piaget/Inhelder 1975, S. 85).
} 


\subsection{Eins-zu-eins-Zuordnungen}

Alle Aufgaben dieser Komponente können mit Zählen, mit Legen oder mit Zuordnungslinien gelöst werden. Es ist spannend zu beobachten, dass die ganze Palette von Lösungsmöglichkeiten ausgenutzt wird, wobei keiner Leistungsgruppe eine bevorzugte Lösungsstrategie zugeordnet werden kann.

31 Kinder haben das einfache Würfelmuster der Aufgabe A11 (siehe Seite 112) mit den vorhandenen Holzwürfeln in gleicher Formation wie die Vorlage neben dieser Vorlage hingelegt, 15 Kinder haben die Holzwürfel direkt auf die schwarzen Punkte der Vorlage gelegt und 19 Kinder haben zuerst die Punkte der Vorlage abgezählt und dann die richtige Anzahl Holzwürfel ungeordnet hingelegt oder rübergeschoben.

In ähnlicher Vielfalt wurde die Aufgabe A12 (siehe Seite 112) gelöst: 30 Kinder haben die Holzwürfel in gleicher Formation wie die Vorlage neben dieser hingelegt, 10 Kinder haben die Punkte der Vorlage durch die Holzwürfel zur Deckung gebracht und 24 Kinder haben zuerst die Punkte der Vorlage abgezählt und dann versucht, die entsprechende Anzahl Holzwürfel aus dem Vorrat abzuzählen. Drei Kinder rechneten die fünf und die sechs Augenzahlen zusammen und zählten dann die richtige Anzahl Holzwürfel aus dem Vorrat. Die Anwendung einer richtigen Strategie garantiert aber noch keinen Erfolg, vor allem wenn die zu den Strategien notwendigen Kompetenzen nicht oder noch zu wenig gefestigt vorhanden sind. So gibt es Kinder, welche die Form falsch oder unvollständig legen. Zum Beispiel schafften drei Kinder nur die Bildung der Fünferformation, bei der Sechserformation scheiterten sie. Noha (II/17.5) ${ }^{55}$ legt zwar die Fünferformation richtig, will anschließend das Sechserbild in eine Reihe legen und verwendet dann aber 7 Holzwürfel. Häufiger kommen Fehler bei der Abzählstrategie vor: es wird einfach zu ungenau gezählt. Die Strategie bei der die Holzwürfel die Vorlagepunkte abdecken, erweist sich als die sicherste: hier wurden keine Fehler gemacht.

Vor allem Kinder der Alterstufe IV und V haben bei der Aufgabe A13 (die Kerzenaufgabe; siehe Seite 113) direkt und ohne irgendwelche ersichtlichen Zählaktivitäten die korrekten Zuordnungsstriche gezeichnet. Die erforderliche Anzahlerfassung erfolgte allem Anschein nach bei diesen älteren Kindern simultan, bei maximal fünf Kerzen resp. Kerzenhaltern ist das auch für unter sieben Jahre alte Kinder gut machbar. Gesamthaft acht Kinder, allesamt aus den Alterstufen I bis III haben hingegen deutlich gezählt; Alex

\footnotetext{
${ }^{55}$ Bei individuellen Lösungen werde ich im Weiteren die Namen der Kinder angeben. Dabei bedeutet II die Altersstufe und 17.5 die erreichte Punktzahl.
} 
(II/50) tippt zählend das erste Bild mit Kerzen an, registriert „drei“, hält den Finger auf diesem Kerzenbild und sucht jetzt auf die Kerzenhalter tippend und zählend das passende Bild. So arbeitet er eine imaginäre Matrix vollständig ab, ohne aber Zuordnungsstriche zu zeichnen. Es gab Kinder, die überfordert waren, so z.B. Yvonne (II/25), die einfach die Kerzenständer anzumalen beginnt oder Nico (II/20), der zu jedem Bild die vorhandene Anzahl Objekte hinschreibt, dann aber nicht weiß, was er damit anfangen soll.

Svenja (IV/87.5) löst die Aufgabe A14 (siehe Seite 114) einfach durch Zählen: Sie beginnt beim unteren Bild, zählte die Eier und dann die Hühner, stellt fest, dass es ein Huhn zuviel hat, geht ohne Kommentar zum mittleren Bild über und wiederholt das Zählen mit der Feststellung, dass es hier gleich viele hat und zeigt dann triumphierend auf dieses mittlere Bild. Auf ähnliche Art haben gesamthaft drei Kinder versucht mit Zählen die Aufgabe zu lösen. Sehr schön ist die Lösung von Anna (IV/65), die die Aufgabe ohne zu zählen auf folgende Weise löst: Sie beginnt schon mal mit dem mittleren Bild, legt dann ein Finger auf das Huhn ganz links auf dem Bild und ein Finger der anderen Hand zur gleichen Zeit auf das Ei darunter und schreitet dann auf diese Art zum weiteren Huhn und Ei nach rechts. So fortfahrend beendet sie die Reihe und es bleibt weder ein Huhn noch ein Ei übrig. Damit weiß Anna, dass das mittlere Bild das richtige ist und sie zeigt dann auch darauf, ohne die übrigen zwei Bilder zu beachten. Anna hat die Aufgabe in speditiver und eleganter Eins-zu-eins-Zuordnungsart gelöst: Einem Huhn wird ein Ei zugeordnet. Dass die meisten Kinder mit dem mittleren Bild beginnen, habe ich bereits auf der Seite 113 versucht zu begründen. Es zeigt sich dann auch oft, dass dieser äußere vertrauenserweckende Eindruck sehr auf die Kinder wirkt ${ }^{56}$. 11 Kinder haben ohne zu zögern und ohne zu zählen auf das richtige mittlere Bild gezeigt. Sabine (IV/77.5) zeigt ebenfalls sofort auf das mittlere Bild mit der Bemerkung: „Beides [sie

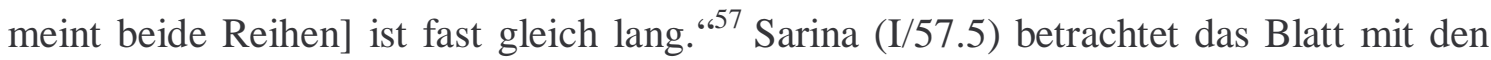
drei Bildern relativ lange (ca. $8 \mathrm{Sec}$ ) und zeigt dann auf das richtige mittlere Bild. Es haben trotz dem ausdrücklichen Hinweis, Striche zeichnen zu dürfen nur 9 Kinder versucht, die Aufgabe mit diesen Zuordnungslinien zu lösen. (Markus (I/50) beginnt im mittleren Bild Zuordnungsstriche zu ziehen. Nach fünf korrekten Linien zögert er, hält inne, hört auf Striche zu ziehen und zählt dann alle Eier, weiß dann aber nicht mehr wei-

\footnotetext{
${ }^{56}$ Zur Erinnerung: Das Verhältnis der Länge der Hühnerreihe zu der der Eierreihe ist im mittleren richtigen Bild am günstigsten.

${ }^{57}$ Alle Äußerungen der Kinder wurden in Schweizer Dialekt gemacht. Zum besseren Verständnis gebe ich aber die Aussagen der Kinder in Standarddeutsch wieder.
} 
ter. Markus hat wohl die Zuordnungsstrategie begriffen und ist auf dem Sprung in die Phase II der Prinzipien-Nachher-Theorie: er möchte das Problem mit Abzählen lösen und scheitert wohl am noch begrenzten Erinnerungsvermögen. Sven (III/37.5) versteht die Aufgabe überhaupt nicht und beginnt das ganze mittlere Bild zu umrahmen ähnlich wie wiederum Yvonne (II/25), die sofort beginnt, die Hühner mit dem Bleistift auszumalen. Sami (IV/35) ist sich nicht sicher, meint aber immerhin, dass es im unteren Bild einfach zu wenig Eier hätte. Diese Aufgabe zeigt auf schöne Art und Weise wie vielfältig die Möglichkeiten sind, welche die Kinder aufgreifen um Probleme anzugehen und im besten Falle auch zu lösen.

Haci (V/82.5) rechnet bei der Aufgabe A15 (siehe Seite 114) auf folgende Weise laut vor, indem er auf das richtige Bild c zeigt: „Fünf und fünf ist zehn und noch fünf dazu ist fünfzehn." Haci, der noch keine sieben Jahre alt ist und noch nie eine Schule besucht hat, löst diese Aufgabe mit einer Sicherheit und Abgeklärtheit, die erstaunt. Es ist sicher, dass viele Kinder auf verschiedene Weise auch im Elternhaus oder im Bekanntenkreis oft gefördert werden. Trotzdem lassen unsere Videoaufnahmen den Schluss zu, dass Kinder solche und ähnliche Strategien aus der Situation heraus spontan selber entwickeln. Es haben noch drei weitere Kinder die Fünfer-Struktur erkannt und ausgenutzt, darunter erstaunlicherweise der sehr junge Roy (I/25). Etliche schwächere Kinder zeigten auf den vollen Kasten d, der ja genauso „voll“ ist wie das Vorlagebild mit den 15 Ballonen. 14 Kinder haben versucht, die Aufgabe mit Zählen zu lösen, 8 davon erfolgreich. Für die jungen Kinder war die Aufgabe zu schwer; sei es, dass sie die Aufgabe nicht verstanden; sei es, dass sie nicht über die nötige Lösungsstrategie-kenntnisse verfügten; sei es, dass sie noch nicht die erforderliche Zählkompetenz besaßen.

Die Kinder lösen typische Eins-zu-eins-Zuordnungsaufgaben auf sehr unterschiedliche Weise: auf konventionelle Art durch paarweise Korrespondenz; durch zählen, wobei die starken Kinder oft dazu neigten, das Zählen zur Problemlösung heranzuziehen und durch den äußeren Eindruck, wobei die schwächeren Kinder sich eher vom äußeren Eindruck leiten ließen. 


\subsection{Reihenfolgen}

Es ist nicht ganz einfach das Lösungsverhalten der Kinder bei der Bewältigung der Reihenfolgenaufgaben zu beschreiben, da bei dieser Komponente bis auf die Fragen der Interviewer fast alles stumm abläuft. Die Aufgabenstellungen verlangen zweimal ein Erkennen einer einfachen Reihenfolge, einmal von groß nach klein, das zweite Mal von klein nach groß. Bei der dritten Frage A18 wird eine ,pseudo"-multiple Seriation vorgelegt (siehe Seite 116): die Fragestellung ist komplex, die Aufgabe ist es durch die Redundanz nicht. In fünf Fällen hatte die Interviewerin die Frage noch nicht einmal fertig formuliert, da zeigten die Kinder schon das richtige Bild. Eine weitere interessante Seriation wird in der Aufgabe A19 (siehe Seite 117) dargelegt. Hier wird eine Reihenbildung nach den zwei Merkmalen Länge des Stocks und Größe des Hundes zugleich verlangt. Hier wird zur Lösung eine Matrix aufgebaut, deren Zeilen und Spalten so organisiert sind, dass jeweils das eine Merkmal (hier die Länge des Stocks) konstant gehalten wird, während das andere Merkmal (hier die Größe des Hundes) mit Hilfe von Paarvergleichen systematisch variiert wird (vgl. zur Oeveste 1987, 34). Es ist bei dieser Komplexität nicht verwunderlich, dass viele jüngere Kinder den Entwicklungsstand für die Lösung dieser Aufgabe noch nicht erreicht haben. Der Kompetenzunterschied der Kinder zwischen dem ersten und zweiten Kindergartenjahr mit 27\%, resp. 57\% richtigen Antworten wird doch sehr deutlich. Zur Illustration sei noch das Verhalten von Kindern beschrieben, welche die Entwicklungsstufe dieser Kompetenz der Serienbildung noch nicht erreicht haben. Neun dieser Kinder haben den Bleistift beim ersten Stock (dem größten) links angesetzt und haben korrekt einen Strich direkt zum darüber liegenden Hund (dem größten) gezeichnet. Dann haben sie den Stift beim nächsten Stock angesetzt und haben den Strich wieder senkrecht nach oben zum darüber liegenden Hund gezogen. So sind sie dann noch zweimal verfahren und waren mit den vier senkrecht gezogenen Strichen (drei falsche) durchaus zufrieden.

Das oben beschriebene Verfahren der Matrixbildung wird auch bei Bewältigung von multiplen Seriationen angewandt und ist sehr effektiv und sicher. Nach Piaget/Szeminska (1975) ist mit der Entwicklung der multiplen Seriation erst ab 7;6 Jahren zu rechnen, wobei diese zeitliche Fixierung kritisiert wurde (vgl. zur Oeveste 1987, 34). Die Erlangung der multiplen Reihenfolgenkompetenz kann nach unseren Untersuchungen (siehe Aufgabe A18 Seite 116) um das Alter von 6;6 Jahren angesetzt werden. 


\subsection{Gebrauch von Zahlwörtern}

Es herrscht allgemeiner Konsens, dass das Aufsagen der Zahlwortreihe ${ }^{58}$ eine Basiskompetenz ist, und - wie wir gesehen haben - für weitere numerische Aktivitäten von zentraler Bedeutung ist. Unsere Untersuchung zeigt, dass sich diese Kompetenz ab dem 4. Lebensalter entwickelt und bei den meisten Kindern vor dem 7. Lebensalter abgeschlossen ist. Die folgende Abb. 61 zeigt sehr schön den prozentualen Anstieg der Zählkompetenz bis zur Zahl 20 in der vorliegenden Schweizer Untersuchung. Zur Erinnerung: I (4;07 - 4;12), II (5;01 - 5;06), III $(5 ; 07$ - 5;12), IV $(6 ; 01-6 ; 06)$ und V $(6 ; 07-$ $6 ; 12)$ und $N=70$.

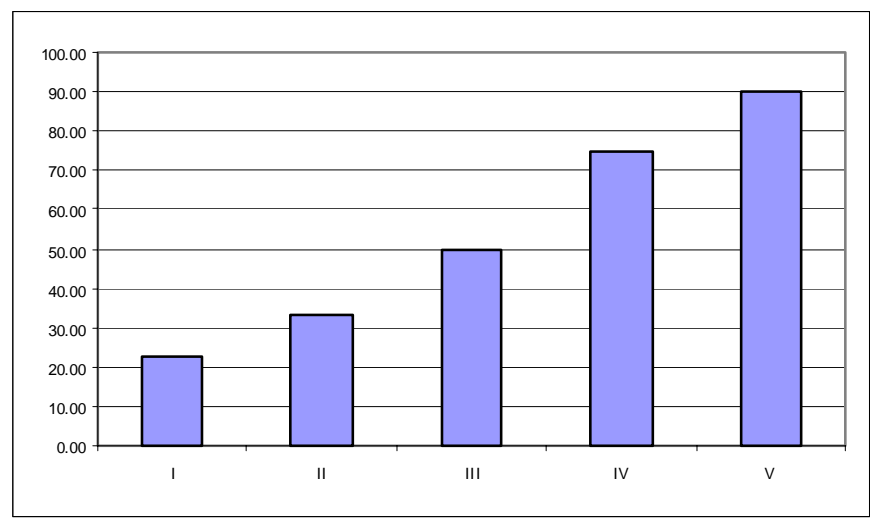

Abb. 61 Anstieg der Zählkompetenz in Abhängigkeit zum Alter.

Nur Christine (IV/20) hat als einziges Kind der ganzen Untersuchung nicht begriffen, worum es beim Zählen geht. Alle übrigen Kinder haben mit den ihnen zur Verfügung stehenden Mitteln versucht, die Folge hinzukriegen.

Da die eigenen Finger die nächstgelegenen und bequemsten numerischen Werkzeuge sind, bedienen sich Kinder ihrer gerne. In der noch unsicheren Phase des Zahlreihenerwerbs können die Finger eine willkommene Stütze sein; aus dem abstrakten Memorieren wird so ein konkretes Handeln im wörtlichen Sinne: einem Finger wird eine Zahl zugeordnet. So haben neun Kinder mit Hilfe ihrer Finger die Zahlreihe aufgesagt, indem sie für jede Zahl einen Finger gestreckt haben. Nicht alle dieser Kinder haben es mit

\footnotetext{
${ }^{58}$ In der mathematikdidaktischen Literatur werden in diesem Zusammenhang die Begriffe Zahlenreihe und Zahlenfolge gleichwertig gebraucht. Dies ist im mathematischen Sinne nicht korrekt, da es sich hier immer um eine Folge handelt. Ich werde mich aber der Konvention beugen und beide Begriffe gebrauchen.
} 
dieser Technik auch tatsächlich ohne Fehler bis 20 geschafft. Ardita (III/40) schafft es nur bis fünf und Noha (II/17.5) ist sich bis 10 ganz sicher, weiß dann aber nicht mehr weiter, genauso wie Eveline (II/60), die ganz verstohlen ihre Finger benutzt. Dass die Zehn gewissermaßen eine natürliche Grenze bedeutet, wird auch bei andern Kindern deutlich, die dann bei elf, zwölf, ... unsicher wirken und Fehler machen wie das Auslassen oder die doppelte Zuweisung von Fingern oder Zahlwörtern. Vier Kinder versuchen durch einen Rhythmus eine Struktur und eine Stütze beim Zählen zu erreichen, so Markus (I/50), der mit gleichmäßigem Klopfen auf den Tisch sein Aufsagen unterstützt. Beide Techniken, Finger und Rhythmus, geben dem ganzen Unterfangen „Zählen bis 20“ ein Korsett, an das man sich halten kann. Dass das Zählen bis 20 im Alter bis 6 Jahren noch nicht eine sehr solide und gesicherte Tätigkeit darstellt, sollen einige Einzelbeobachtungen dokumentieren. Yvonne (II/25) zählt korrekt und flüssig bis 14, stockt dann, beginnt wieder bei 1, schafft es wieder bis 14 und gibt dann auf, ähnlich wie Sarina (I/57.5), die leise und korrekt bis 15 zählt und dann nicht weiter weiß, oder Marco (II/45), der wie folgt die Zahlenreihe aufsagt: 1, 2, 3, 4, 5, 6, 7, 8, 9, 10, 7, 8, 9, 10, 7, 8, 9, 10. Michelle (II/15) schafft es gar nur bis 7, dann wird es wirr: 1, 2, 3, 4, 5, 6, 7, 1, 5, 4. Sami (IV/35) kennt offenbar die Zahl 18 nicht; bei allen Zählversuchen lässt er ganz konsequent die Zahl 18 aus. Wie die Beispiele zeigen, ringen die Kinder nach der richtigen Form der Zahlenfolge; durch vielfältige Aktivitäten sollte dieser Prozess schon im Kindergarten unterstützt und gefördert werden. Erst auf einer gefestigten Zahlvorstellung und auf der Basis einer korrekten Kenntnis und einem einwandfreiem Gebrauch der Zahlenfolge öffnet sich ein Fenster zur vielfältigen Welt der Mathematik. Vielfach sind erst ausgehend von dieser Kompetenz mathematische Situationen durchblickbar und lassen sich numerische Aufgaben und Probleme lösen. Die Fehlerhäufigkeit ist dann auch bei Problemlösungen mit Zählstrategien geringer als bei Lösungsversuchen auf Grund von Mustern oder von äußeren Eindrücken.

Dies zeigt sich auch bei der Aufgabe A22 (siehe Seite 121), bei der ein Kasten mit sieben Punkten zu zeigen ist. Etwa gleich viele Kinder haben die Aufgabe mit Zählen einerseits oder mit Ausnutzung des Würfelmusters $(6+1=7)$ andererseits zu lösen versucht. Ilona (I/82.5) zeigte auf Basis der Mustererkennung sofort auf das richtige Bild, hat dann aber doch zur Sicherheit nachgezählt; sie vertraute offenbar der Zählstrategie mehr als ihrer ersten spontanen klugen Methode. Während bei den leistungsstarken Kindern beide Strategien zum Erfolg führten, ist die Fehlerquote der Zählstrategie bei den weniger leistungsstarken Kindern weit geringer als bei Kindern, die versuchten, die 
Aufgabe auf Grund ihres Musters oder äußeren Eindruckes zu lösen. Es ist durchaus denkbar, dass bei diesen Kindern das Kardinalitätsprinzip noch nicht gefestigt ist und sie den Sinn des Zählens noch nicht genau verstanden haben, oder aber umgekehrt, dass es Kinder darunter gibt, welche die Aufgabe raffiniert lösen wollten, indem sie versuchten auf bekannte Muster zurückzugreifen und diese auf die neue Situation anzuwenden und darum auf das sichere Zählen verzichteten.

Dass Zählstrategien auch bei Ordinalitätsaufgaben sicher zum Ziel führen, wissen die meisten Kinder, so zählen auch fast alle bei der durch die Unstrukturiertheit der langen Blumenreihe schwierigen Aufgabe A24 (siehe Seite 121) munter darauflos. Es gibt Kinder, die offenbar noch nie mit der Ordinalität aktiv zu tun hatten, so Melinda (IV/85), die zuerst völlig ratlos die Blumenreihe betrachtet und offenkundig die Aufgabe nicht versteht. Nach einigem Zögern beginnt sie aber zu begreifen und zählt die Blumen ab und zeigt mit dem Bleistift, den sie nahe vor ihrem Gesicht in ihrer Hand hält, aus dieser Entfernung auf die gezählten Blumen, merkt, dass sie sich verzählt hat, beginnt wieder von vorne und verzählt sich abermals. Jetzt erst tippt sie mit ihrem Bleistift die gezählten Blumen an, ordnet den berührten Blumen das richtige Zahlwort zu, kann so die gesuchte achtzehnte Blume zeigen und löst somit das Problem mit Bravour. Das Wissen um die Ordinalität wurde hier direkt aus einem Problemkontext heraus erschlossen, ganz im Sinne der situierten Kognition (siehe Kapitel 5.2).

Anna (IV/65), die die Ordinalität ebenfalls wie einige andere Kinder auch nicht verstanden hat, ist nicht in der Lage, aus der Situation heraus, so wie Melinda, die erforderlichen Schlüsse zu ziehen und das Problem zu lösen. Sie schaut ganz entrüstet die Interviewerin an und äußert sich unwillig: „Ich verstehe das nicht“ und gibt sofort auf. Auch Andreas (V/55) und Kilian (V/38) sind ratlos; diese zwei Buben zeigen dann aber nach einiger Zeit mit Bestimmtheit auf die falsche letzte Blume. Alle in diesem Zusammenhang erwähnten Kinder sind etwas gleich alt und können bis 20 zählen, aber nur Melinda als leistungsstärkstes Kind mit 85 Punkten war in der Lage, aus der Situation heraus das Problem der Ordinalität zu lösen. Hier zeigt sich wiederum eindrücklich die Abhängigkeit der Problemlösekompetenz von der allgemeinen Leistungsfähigkeit. Während die weniger leistungsfähigen Kinder bei dieser neuen Problemstellung kapitulierten oder einfach ins Blaue rieten, ist die leistungsstarke Melinda in der Lage, die Situation zu analysieren und das Problem zu lösen. Zu erwähnen sind noch Yvonne, die nur bis 14 zählen kann und Melina (V/45), die die Zahlen nur bis 12 kennt, sowie Sami, dem - wie schon erwähnt - die Zahl 18 nicht bekannt ist. Alle drei Kinder haben alles begriffen 
und lösen alle folgenden Aufgaben mit ihren Voraussetzungen „korrekt“. Yvonne tippt die ersten 14 Blumen an und nennt korrekt die entsprechenden Zahlwörter, stockt dann, weiß dass es ein Blume weiter rechts sein muss, kann sie aber nicht zeigen, weil sie die entsprechenden Zahlwörter nicht kennt und Melina verhält sich genauso bis zur Zahl 12. Sami hat die Zahl 18 nicht in seinem Zahlenrepertoire, also gibt es für ihn diese 18. Blume nicht und er zählt darum folgerichtig die ganze Reihe der Blumen ab ohne die 18. zu benennen und zu zeigen. Ein wenig erstaunen mag die folgende Tatsache, dass es von bei dieser Aufgabe erfolgreichen 17 Kindern mit 7 Kinder weniger Kinder gibt, die bei der Auszählung auf die Blumen getippt haben als die 10 Kinder, die die Blumen nur mit den Augen fixierten und dem entsprechenden Zahlwort zugeordnet haben.

\subsection{Strukturiertes Zählen}

Die Bestimmung der Anzahl Elemente einer Menge wird von den Kindern mit großer Selbstverständlichkeit mit Zählen dadurch erreicht, dass jedem zu zählenden Element eine Zahl zugeordnet wird und die letzte dieser zugeordneten Zählzahlen gibt als Kardinalzahl die zu ermittelnde Anzahl Elemente wieder. Nur fünf Kinder haben noch Schwierigkeiten mit einer korrekten Zuordnung, darunter Christine (IV/20), die die Phase I der Prinzipien-Nachher-Theorie noch nicht erreicht hat und dadurch den zu zählenden Elementen nicht die korrekte Zählzahl zuordnen konnte. Die übrigen vier Kinder haben wohl schon eine vage Vorstellung des Kardinalitätprinzips, sind aber nicht im Stande, das Zeigen und das Zuordnen der Zählzahl richtig zu koordinieren.

Es ist sehr hilfreich, die gezählten Elemente zu kennzeichnen, sei es durch räumliche Umordnung oder durch strukturiertes Abarbeiten (zum Beispiel von links nach rechts oder von unten nach oben) oder durch andere Kennzeichnungen. Bei der Aufgabe A26 (siehe Seite 124), bei der 16 Holzwürfel in vier Reihen zu je vier Holzwürfel mit etwas Abstand auf dem Tisch liegen, eruieren 25 Kinder diese Anzahl durch schlichtes Antippen der Holzwürfel. Ein herumschieben der Holzwürfel ist durch die regelmäßige und überblickbare Anordnung der Holzwürfel nicht unbedingt nötig. Wohl darum bevorzugen vor allem die älteren und leistungsstarken Kinder das Antippen ohne herumschieben, 15 Kinder schieben die Holzwürfel zur Seite, wobei der Fehleranteil dieser Strategie weit höher ist als bei der Gruppe, die die Holzwürfel nur antippen: $72 \%$ der „Tipper“ 
haben Erfolg, hingegen nur 53\% der „Verschieber“. Das ist aber sicher die Konsequenz der eben gemachten Feststellung, dass vor allem die Leistungsstarken das Tippen bevorzugen. Es ist noch zu erwähnen, dass nur vier Kinder versucht haben, die Aufgabe ohne Zählen zu lösen: Florian (II/82.5) stellt zwar fest, dass es 8 und 8 Holzwürfel sind, kann dann aber die Summe nicht nennen, also zählt er halt doch ab. Auch Haci (V/82.5) versucht es auf die raffinierte Art, sieht die Struktur, scheitert aber bei der korrekten Bestimmung der Summe.

Es erfordert höchste Konzentration, die Anzahl einer Kollektion von Elementen zu bestimmen, die ohne Anfang und Ende angeordnet ist. Bei der Aufgabe A27 (siehe Seite 124), bei der 9 Holzwürfel in einem Kreis mit etwas Abstand zwischen den Holzwürfeln angeordnet sind, tritt denn auch der erwartete Fehler gehäuft auf: die doppelte Berücksichtigung, resp. das Auslassen von Elementen. Viele Kinder, vor allem die leistungsstarken, sind sich der Schwie-rigkeit bewusst und konzentrieren sich auf das erste der gezählten Elemente, um es ja nicht noch einmal zu zählen. So beginnt zum Beispiel Melinda (IV/85) einfach darauflos zu zählen, hält dann aber ruckartig innen, weil sie die Schwierigkeit bemerkt, und beginnt wieder, diesmal voll konzentriert, von vorne. Anna (IV/65) tippt die Holzwürfel an, merkt dann, dass da etwas schief gehen könnte, hält dann den ersten gezählten Holzwürfel fest, das scheint ihr auch noch unsicher, so dass sie schließlich die gezählten Holzwürfel beiseite schiebt und so sicher zum Erfolg kommt. Auf sicher geht Michael (IV/87.5), der einfach einen Finger auf den zuerst gezählten Holzwürfel legt und diesen Würfel bis zum Schluss des Zählprozesses festhält oder Eveline (II/60), die hinter dem ersten gezählten Würfel einen Finger auf den Tisch ausstreckt, gewissermaßen als Separator zwischen gezählten und noch zu zählenden Holzwürfeln und diesen Finger da belässt, bis sie fertig gezählt hat. Die Anzahl der Kinder, die mit Verschieben der Holzwürfel, und die Anzahl der Kinder, die nur mit Antippen dieser Würfel zum Erfolg kommen, liegt beide Male bei etwa 25, wobei die leistungsstarken Kinder eher das Antippen ohne Verschiebung bevorzugen, wohl weil eine regelmäßige Struktur in Form eines Kreises gegeben ist. Die übrigen Kinder haben ihre Hände für das Abzählen nicht zu Hilfe genommen und haben dadurch auch eine sehr hohe Fehlerquote.

Hinlänglich vorgestellt worden ist die Aufgaben A28 (siehe Seite 125), bei der 20 Holzwürfel in unregelmäßiger Anordnung auf einem Haufen abgezählt werden müssen. Vor allem die leistungsstarken Kinder haben eine Sicherheit des korrekten Abzählens durch Verschiebung der Holzwürfel erreicht. Die Fehlerquote bei dieser Strategie ist 
dann auch wesentlich geringer als bei den Kindern, die die Holzwürfel lediglich angetippt haben. 21 von den 28 „Verschiebern“ haben die korrekte Anzahl Würfelchen eruiert, hingegen nur 6 von den 22 Kindern, die lediglich die Würfelchen angetippt haben. Die Leistungsfähigkeit zeigt sich also auch in der Wahl der richtigen Strategie, das heißt, die Kinder müssen die Schwierigkeit und Fußangeln einer Aufgabe im Voraus abschätzen können, um die Erfolg verheißende Strategie aus ihrem verfügbaren Repertoire zu wählen. Die Kinder, meist die leistungsschwachen, die auf das Verschieben oder auf das Antippen verzichteten, haben einen ungemein höheren Fehleranteil. Das bedeutet nun aber, dass neben der Zählkompetenz auch die richtige Einschätzung der Aufgabenschwierigkeit ein Garant für Erfolg bedeutet. Bei dieser Aufgabe A28, so könnte man erwarten, haben Kinder, die die Zahlfolge bis 20 nicht beherrschen, keine Chance diese Menge von 20 Holzwürfeln abzuzählen. Im Folgenden gebe ich nun aber Interviewsequenzen wieder, die zeigen, dass Kinder sehr wohl eine Kollektion von 20 Elementen bestimmen können, auch wenn sie die einfache Zahlwortfolge bis 20 ohne Kontext vorher nicht aufsagen konnten.

\section{Aufgabe A21}

Interviewer: Zähle bis zwanzig.

Sarina (I/57.5): (Sie beginnt zaghaft und zählt sehr langsam und leise.)

\section{$1,2,3,4,5,6,7,8,9,10,11,12,13,14,15$.}

(Sie wartet sehr lange.)

\section{Ich weiß nicht mehr weiter.}

\section{Aufgabe A28}

Interviewer:

Sarina:

\section{Zähle diese Holzwürfel.}

(Sie schiebt die gezählten Holzwürfel zaghaft, fast schüchtern zur Seite.)

\section{$1,2,3, \ldots, 14,15,16,17,18,19$.}

(Hier bricht sie ab und wartet vor dem letzten noch nicht verschobenen, also noch zu zählendem Würfel. Es scheint ihr sehr unwohl zu sein. Nach geraumer Zeit gibt sie auf.)

\section{Ich weiß nicht mehr weiter.}


Zwei Beobachtungen sind festzuhalten: Sarina kennt offensichtlich das Zahlwort „Zwanzig“ nicht und ist darum außerstande, die Aufgabe fertig zu lösen; und obwohl sie die Zahlwortfolge bei der Aufgabe A21 nur bis 15 schaffte, gelingt es ihr hier ohne Mühe, ihre Performanz zu steigern und ihre wahre Kompetenz zu zeigen. Erst die konkrete Handhabung mit Materialien, das Herumschieben von Holzwürfeln aktiviert offenbar das Erinnerungsvermögen. Durch diese Performanzsteigerung kann zudem die Kompetenz beeinflusst werden in dem Sinne, dass in kommenden ähnlichen Situationen vermutlich die Zahlwortfolge sicherer und mit höherer Performanz aufgesagt werden kann.

Aufgabe A21

Interviewer: Zähle bis zwanzig.

Marco (II/45): (Er zählt langsam.)

$1,2,3,4,5,6,7,8,9,10,7,8,9,10,7,8,9,10$.

(Er wartet, schaut dann ein wenig gelangweilt herum und zuckt dann hilflos mit den Schultern und gibt auf.)

Aufgabe A28

Interviewer: Zähle diese Holzwürfel.

Marco: (Er tippt die Holzwürfel an und ordnet die korrekte Zählzahl zu.) $1,2,3,4,5,6,7,8,9,10,11,12,13,14,15,16,17,18,19,20$.

Marco kann offensichtlich doch zählen. Auch hier ist das vorliegende Material der Auslöser für diese erstaunliche Performanzsteigerung.

Aufgabe A21

Interviewer: Zähle bis zwanzig.

Eveline (II/60): (Sie zählt langsam und bedächtig und braucht ganz heimlich ihre Finger.)

$1,2,3,4,5,6,7,8,9,10$.

(Sie wartet ein kleines Weilchen, schaut dann keck den Interviewer an, verschränkt ihre Arme vor die Brust und verkündet:)

\section{Weiter kann ich nicht.}

Aufgabe A28

Interviewer: Zähle diese Holzwürfel. 
Eveline: $\quad$ (Sie tippt die Holzwürfel an ohne dass sie sie verschiebt und zählt laut.)

\section{$1,2,3,4,5,6,7,8,9,10,11,12,13,14,15,16,17,18,19,20$.}

Diese Sequenzen sind wieder Bestätigung dafür, dass konkrete Hilfestellungen in unterschiedlicher Form (Finger, Rhythmus, Materialien) sehr hilfreich für Performanzsteigerung und schlussendlich auch für die Erweiterung der Kompetenzen anzusehen sind. Konkrete Materialien sind mit den Fingern und Händen zu fassen, man kann damit hantieren und sinnliche Erfahrungen sammeln und man kann sich auch, wenn mal keine Materialien zur Verfügung stehen, immer wieder an diese sinnlichen Eindrücke zurückerinnern, bis die Handlungen so verinnerlicht sind, dass auf die Materialien ganz verzichtet werden kann.

Wenn keine Hilfsmaterialien zur Stelle sind, so bedienen sich die Kinder schnell einmal ihrer Finger. So haben bei der Aufgabe A29 (siehe Seite 126) sechs Kinder ihre Finger zu Hilfe genommen, indem sie die Anzahl der beiden Würfelbilderpunkte als Finger ausstreckten. Drei dieser Kinder konnten aber trotzdem nicht die richtigen Zahlen, geschweige die Summe nennen. Das regelmäßige und wohl vertraute Würfelmuster mit vier und fünf Augen ist derart einprägend, dass man sich relativ gut an das Bild erinnern kann. Bei sieben Kindern ist es offensichtlich, dass sie ein mentales Bild der Situation präsent haben und dann die Punkte in Ruhe abzählten, so Anna (IV/65) die die Punkte in der gleichen Form wie auf der Vorlage mit dem Finger auf den Tisch tippt und laut bis neun zählt: das zählende Rechnen als Lösungsstrategie einer Additionsaufgabe. Es gibt vermutlich noch mehr Kinder, die so verfuhren, da man aber nicht nachgefragt hat, bleibt es bei dieser Vermutung. Die folgende Interviewsequenz zeigt deutlich, dass eine Befragung auch einen großen Lerneffekt aufweisen kann, weg vom zählenden Rechnen hin zu denkendem Rechnen.

Aufgabe A29

Interviewer: $\quad$ Ich gebe dir ein Bild, das du kurz anschauen sollst.

(Der Interviewer zeigt dem Kind für 2 Sekunden ein Bild mit zwei Würfeln mit den Augenzahlen 4 und 5 und nimmt dann das Bild wieder weg.)

Wie viele Punkte waren es zusammen? 
Sarah (V/55): (Sie zählt leise aus der Erinnerung die neun Würfelaugen, wobei sich ihre Lippen bewegen.)

9

(Sie streckt dann mit der einen Hand vier Finger und mit der andern Hand fünf Finger und schaut sich die beiden Hände lange an.)

Vier und fünf.

(Sie stutzt und erinnert sich offenbar an die vorher geäußerte Zahl neun, zeigt mit dem Kopf auf ihre Hände mit den ausgestreckten Fingern und beginnt zu strahlen:)

\section{Vier und fünf; das ist ja neun!}

Dem Problem des Rückwärtszählens sind etliche Kinder ausgewichen, indem sie die vorgelegte Reihe mit 17 Holzwürfelchen der Aufgabe A30 anstatt wie üblich von links nach rechts (von vorne nach hinten) einfach von rechts nach links (von hinten nach vorne) bei eins beginnend abzählten in der Meinung, dies sei rückwärts, was vom geometrischen Standpunkt aus durchaus vertretbar ist. Die meisten Kinder waren aber rat- und auch hilflos; sie verstanden die Aufgabe nicht. Nur einige wenige leistungsstarke Kinder haben die Aufgabe gelöst und bemerkenswert ist hier die Leistung von Svenja (IV/87.5), die die Zahlenfolge offenbar nicht frei rückwärts aufsagen kann, sondern sich diese Folge im Moment konstruiert: sie merkt sich die 17, zählt dann stumm vorwärts bis 17 und merkt sich die Zahl vor der 17, die 16, nennt und merkt sich diese Zahl 16 und beginnt wieder stumm von vorne an zu zählen bis 16, erinnert sich an den Vorgänger 15, nennt und merkt sich diese Zahl usw. So ist sie in der Lage die Folge rückwärts aufzusagen, wobei das Aufsagen immer schneller vor sich geht.

\subsection{Ergebnisbezogenes Zählen}

Es ist für kleine Kinder schwierig, mehrere Informationen gleichzeitig zu verarbeiten. So haben neun Kinder, vor allem die Leistungsschwachen, bei der Aufgabe A31 während der Reihenbildung vergessen, dass nicht alle, sondern nur 11 Holzwürfelchen zu legen sind, und legen dann fälschlicherweise alle vorhandenen 15 Holzwürfel. Offenbar 
ist die Speicherkapazität der leistungsstarken Kinder größer und auch das macht ihre höhere Leistungsfähigkeit aus.

Ein Beispiel einer noch sehr wagen Vorstellung einer Zuordnung Gegenstand - Zahlwort liefert Nico (II/20): Er holt sich mit einem Finger jeder Hand einen Holzwürfel und legt die beiden Holzwürfel korrekt in die Reihe, zählt dann aber nur ein Zahlwort weiter und beachtet die falsche 2 zu 1 Zuordnung nicht. Dass er mit dieser Technik, er macht noch einen weiteren Fehler, nur bis sieben kommt, kümmert ihn nicht.

Das Berühren der Holzwürfel ist bei allen folgenden Aufgaben dieser Komponente nicht gestattet. Um trotzdem eine Struktur in das Zählen hineinzubringen, bedienen sich zehn Kinder eines Rhythmus'. Das ist hilfreich; bis auf einen Fall hat diese Stütztechnik zum Erfolg geführt. Dass Kinder phantasievoll bei Lösungsansätzen sind, belegt die folgende Sequenz: Ilona (I/82.5) beobachtet die Interviewerin beim Abräumen der Aufgabe A32, bei der 20 Holzwürfel gebraucht wurden und bei der Bereitstellung der Aufgabe A33 ganz genau. Auf die Frage der Interviewerin, wie viele Holzwürfel auf dem Tisch liegen, fragt Ilona zurück: „Wieviel hast du weggenommen?“ und subtrahiert ohne auf die Antwort zu warten von den 20 die weggenommenen 5 Holzwürfel und nennt dann als Resultat 15. Dieses richtige Resultat ist zustande gekommen, ohne dass das Mädchen die zu zählenden Holzwürfel speziell beachtet hätte. Ilona ist zum Zeitpunkt des Interviews keine fünf Jahre alt und hat noch keinen Mathematikunterricht genossen. Dass das vorgelegte regelmäßige Muster zu Erhöhung der Performanz führt, belegt Melina (V/45), die - wie im vorhergehenden Kapitel erwähnt - nur den Zahlbereich bis 12 benutzt und beherrscht. Sie zählt hier konzentriert und laut über die 12 hinaus und nennt das richtige Resultat 15. Bei der folgenden Aufgabe A34, bei der 19 Holzwürfel auf einem Haufen zu zählen sind, schafft sie ebenfalls 15 Holzwürfel korrekt, gibt dann aber nach der Nennung des 15. Würfelchens auf. Hier sehen wir nochmals eine Rückkoppelung Performanz - Kompetenz. Auch Eveline und Marco haben ihre neu erworbene Performanz gefestigt und scheitern bei der Aufgabe A34 nur darum, weil sie einen Holzwürfel doppelt gezählt haben, die Zahlfolge bis 20 haben sie korrekt aufgesagt. Die Basis und Voraussetzung für korrektes zählendes Rechnen ist die Kompetenz, eine Zahlenfolge fehlerfrei aufzusagen. Mit dieser Grundlage sind schon Kinder unter sechs Jahren in der Lage, schwierige Additionsaufgaben zu lösen. Die Aufgabe A35 (siehe Seite 130), bei der die Interviewerin erst fünf Holzwürfel und dann kurz darauf nochmals sieben Holzwürfel unter ihrer Hand verschwinden lässt, erfordert eine gewaltige Gedächtnisleistung der Kinder, eine rasche Situationsanalyse sowie ein rasches Bereitstellen 
einer Lösungsstrategie. Elf Kinder benutzten ihre Finger; sie haben die Aktivität der Interviewer nochmals durchgespielt und haben dann ihre Finger abgezählt. Dass nur zehn Finger gleichzeitig zur Verfügung stehen, hat diesen Kindern keine Probleme bereitet. Anna (IV/65) klopft erst mal fünf Finger der einen Hand leicht auf den Tisch und fährt dann mit der anderen Hand weiter und nimmt dann zum Schluss wieder die erste Hand für die letzten zwei Zahlwörter elf und zwölf. Meral (IV/62.5) sieht sich die beiden Hände mit den fünf gestreckten Fingern lange an, wobei die linke Hand immer wieder zwei und dann fünf Finger zeigt und nennt dann ohne zu zählen 12. Offenbar hat er die „Kraft der Fünf“ (Wittmann/Müller 1990, 32) ausgenutzt und das Ergebnis ohne Zählen erhalten. Es fällt auf, dass viele Kinder, obwohl sie die Nützlichkeit der Finger bei der Lösung von Zählaufgaben sowie numerischer Problemen wohl einsehen, dieses Werkzeug nur verschämt und so gut es geht versteckt benutzen. Man kann sich fragen, ob da Auswirkungen von althergebrachten Vorstellungen bezüglich des Gebrauchs von Fingern aus dem Elternhaus, dem Bekanntenkreis oder sogar der Kindergärtnerin feststellbar sind.

\subsection{Allgemeines Zahlwissen}

Wie bewältigen Kinder alltägliche Situationen, bei denen sie mit Zahlen konfrontiert werden? Können sie ihr zur Verfügung stehendes Repertoire an Wissen und Kompetenzen jederzeit einsetzen? Es zeigt sich, dass Kinder, die wohl über Zählkompetenz verfügen, sich schon bei „einfachen“ Anforderungen irritieren lassen. Zum Beispiel Aufgabe A36 (siehe Seite 131) bei der gesagt wird, dass sich neun Bonbons in der schwarzen Kiste und dreizehn Bonbons in der weißen Kiste befinden. Auf die Frage der Interviewer, in welcher Kiste sich die meisten Bonbons befinden, lassen sich immerhin 8 Kinder von der sehr dominanten schwarzen Kiste in die Irre führen und zeigen auf diese falsche, obwohl vier dieser acht Kinder die Zählaufgabe A21 einwandfrei lösten. Die Subtraktionsaufgabe A37 (siehe Seite 132) wurde schon vorgestellt. 10 Kinder haben eindeutig die Struktur der Ausgangslage und der vorgegebenen Lösungsbilder miteinander verglichen und 9 von diesen Kindern haben auch das richtige Bild, natürlich ohne zu rechnen oder zu zählen, gezeigt. Sehr deutlich macht dies Florian (II/82.5) vor: Er deckt im Ausgangsbild kurzerhand die erste der drei Reihen ab und zeigt dann mit dem Finger 
auf das jetzt modifizierte Ausgangsbild und auf das jetzt identische Lösungsbild. Diese Strukturerkennungstechnik haben die leistungsstarken Kinder angewendet. Einige wenige haben im Ausgangsbild die Punkte gezählt, ohne die wegfallenden drei zu berücksichtigen und haben dann bei den vorgeschlagenen Bildern oft ohne zu zählen das richtige Bild gezeigt. Die Sechs ist dann wohl für diese Kinder durch die Struktur der zwei Dreierreihen sofort ersichtlich. Es sei hier zum wiederholten Mal erwähnt, dass viele leistungsschwache Kinder mit derartigen Aufgaben nicht zu Rande kommen und gar nicht wissen, um was es hier überhaupt geht. So eine für viele verwirrende Aufgabe ist die Hühneraufgabe A38 (siehe Seite 132), bei der zu den acht Hühnern noch zwei dazugekauft werden und die Kinder dann das Bild mit der richtigen Anzahl Hühner zeigen sollen. Ein Schritt weiter als auf dem Stand des totalen Unverständnisses ist Alex (II/50), der immerhin merkt, dass es nach der Transaktion des Dazukaufens mehr Hühner hat und dann, vielleicht um ganz sicher zu gehen, auf das Bild mit den meisten Hühnern zeigt: „Hier, weil es hier am meisten hat.“ Diejenigen Kinder, die bei dieser Aufgabe erfolgreich waren, haben in einem ersten Schritt etwa zu gleichen Teilen die beiden Strategien des Abzählens und des Rechnens benutzt. Damit haben sie die Information, dass es dann 10 Hühner hat in den unteren Bildern durch einfaches Auszählen und Vergleichen umgesetzt. Von den wenigen durchwegs leistungsstarken Kindern, die die letzte Aufgabe A40 (siehe Seite 134) gelöst haben, gibt es ebenfalls zu gleichen Teilen Zähler und Rechner, die dann mit der entsprechenden Strategie die Zahl 11 eruierten und dann auf das entsprechende Feld zeigten.

\subsection{Zusammenfassung}

Kinder im Alter zwischen viereinhalb und sieben Jahren zeigen ein einheitliches Lösungsverhalten bei Vergleichskonzepten und Klassifizierungen, woraus sich ableiten lässt, dass die Entwicklung dieser Teilleistungen ganz in Übereinstimmung der Folgeuntersuchungen von zur Oeveste $(1987,32)$ im Alter bis 7 Jahren abgeschlossen ist. Dem Zählen wird von leistungsstarken Kindern bei der Lösung von vielen mathematischen Aufgaben das höchste Vertrauen entgegengebracht in dem Sinne, dass diese Kinder sich mit dieser Technik in höchster Sicherheit wähnen. Dem Zählen wird offenbar die höchste Autorität zugebilligt. Falls es die Situation zulässt, neigen darum leistungsstarke Kin- 
der dazu, bei Zuordnungsaufgaben mit Hilfe ihres Zählvermögens die Aufgaben zu lösen, wohingegen sich die weniger leistungsstarken Kinder oft nur von äußeren Eindrücken leiten lassen und sich häufig von subjektiven Sinneseindrücken irritieren lassen. Die Eins-zu-eins-Korrespondenz wird von fast allen Kindern da angewandt, wo das Zählen nicht möglich ist; auch in diesem Vergleich ist es auffällig, dass die leistungsstarken Kindern viel gewandter und sicherer mit dieser Technik umgehen. Leistungsschwache Kinder tun sich bei der Koordination der Zuordnung erstes Objekt - zweites Objekt oft schwer. Einerseits, weil die Motorik noch nicht überall fertig ausgereift ist, andererseits aber vor allem darum, weil die Eins-zu-eins-Zuordnung, wie unsere Untersuchungen zeigten (siehe ab Seite 192), noch nicht sicher verfügbar ist. Im Allgemeinen sind im Lösungsverhalten der Kinder bei Aufgaben im Themenkreis der Reihenfolgen keine prinzipiellen Unterschiede feststellbar. Ganz in Übereinstimmung mit der Entwicklungshierarchie von Piaget/Szeminska (1975) sind hier die älteren Kinder sicherer im Umgang mit neuen Situationen und im Durchschnitt kompetenter als die jüngeren Kinder, was sich aber im grundsätzlichen Lösungsverhalten nicht bemerkbar macht. Auffällig ist beim Gebrauch der Zahlwörter, das leistungsstarke Kinder schon in der Lage sind, Situationen sofort zu überblicken und sich schnell für eine geeignete Lösungsstrategie aus ihrem Repertoire zu entscheiden; sei es durch direktes einfaches Zählen der Objekte, durch strukturiertes Zählen oder durch Mustererkennung. Weniger leistungsstarke Kinder bekunden vor allem Schwierigkeiten, die noch unsicher verfügbare und noch nicht jederzeit fehlerfrei abrufbare Zahlwortreihe in neuen Situationen spontan und sicher einzusetzen ${ }^{59}$. Sehr offensichtlich ist das größere Lösungsrepertoire der leistungsstarken Kinder beim strukturierten Zählen. Vor allem in neuen Situationen, bei denen die Schwierigkeit der Aufgabe nicht von vornherein feststellbar ist, zeigen Kinder mit höherem Leistungsvermögen sehr viel flexiblere Handhabung ihrer Möglichkeiten; sei es durch konsequentes Berühren der zu verarbeitenden Objekte, sei es mit Strukturierung durch Ortsänderung der Objekte oder durch Separation der zu zählenden Objekte. Viele „einfache“ Fehler treten bei leistungsstarken Kindern gar nicht auf, wie ungenaue Zuordnungen, Auslassungen von Objekten oder Zählzahlen, vor allem weil sie sich der Fußangeln sofort bewusst sind oder diese im Laufe der Bearbeitung der Aufgabe zur Kenntnis nehmen und entsprechende Schlüsse ziehen. So sind sich viele leistungsstarke

\footnotetext{
${ }^{59}$ Es soll an dieser Stelle nochmals betont werden, dass die Leistungsfähigkeit nicht immer mit dem Alter korrespondiert; sehr junge Kinder (Alterstufe I und II) gehören ebenfalls zu den leistungsstarken Kindern und umgekehrt sind Kinder der Alterstufe (IV und V) auch bei den leistungsschwachen Kindern anzutreffen.
} 
Kinder nicht „zu schade“, auch bei vermeintlich „einfachen“ Aufgaben zur Sicherheit Hilfsmittel einzusetzen, wohingegen die Kinder aus dem andern Leistungsspektrum sich öfters überschätzen und meinen, ohne Hilfen direkt und schnell zum Ziel zu kommen. Beim ergebnisbezogenen Zählen fällt auf, dass die Speicherkapazität der Kinder für Erfolg oder Misserfolg direkt verantwortlich zeigt. Es sind viele eher leistungsschwache Kinder zu beobachten, die bei der Bearbeitung von mehrschichtigen Problemen das Ziel aus den Augen verlieren; zum einen, weil sie müde sind und ihre Konzentrationsfähigkeit nachlässt, zum andern, weil ihr Erinnerungsvermögen noch nicht so ausgereift ist, um alles gleichzeitig präsent zu haben. Beim allgemeinen Zahlwissen sind Strukturerkennungen von Vorteil. Kinder mit hoher Gesamtpunktzahl bedienen sich dieser Technik, wo immer es geht, und das Zählen wird dann gewissermaßen als „Qualitätskontrolle“ am Schluss noch eingesetzt.

Vor allem eine hohe Zählkompetenz, die Möglichkeit von Muster- und Formerkennung, differenzierte Wahrnehmungsmöglichkeit, schnelle Analyse von Problemstellungen, breites Repertoire von Problemlösestrategien, Raumorientierung sowie hohe Speicherkapazität des Gedächtnisses sind eine gute Basis für hohe Leistungsfähigkeit eines Kindes in der Mathematik. Um eine fundierte vorschulische Förderung im mathematischen Bereich zu gewährleisten, sind vor allem auch die folgenden Aspekte zu berücksichtigen ( siehe Hasemann 2003, 38):

Einsicht in die Invarianz von Mengen, Sicherheit in der Eins-zu-eins-Zuordnung, in den Bereichen Klassifikation und Reihenfolgen. Diese Kompetenzen entwickeln sich bei 4- bis 6-jährigen Kindern parallel zum Zählvermögen und diese beiden Bereiche scheinen sich gegenseitig zu bedingen (siehe Seite 186).

Die Strategien der Kindergartenkinder beim elementaren Rechnen, Zählen und bei Zahlzerlegungen sind noch oft an realen Gegenständen und Bilder gebunden und werden weniger als abstrakte Operationen verstanden. Bei Vergleichen dominiert zudem noch der direkte, optische Eindruck.

Wie bereits erwähnt, werden im Kapitel 10.2 Unterrichtsbeispiele aus Schweizer Kindergärten aufgeführt, bei der viele der formulierten Ideen und Anregungen wieder zu erkennen sind. 


\section{Vorschulische Förderung und Basisstufe}

\subsection{Leistungsdifferenzen}

In den vorangegangenen Kapiteln wurde gezeigt, dass es enorme Leistungsunterschiede innerhalb einer Jahrgangsstufe gibt. Die zwei folgenden kleinen Tabellen mögen das exemplarisch an je vier Kindern nochmals illustrieren. Die erste Tabelle zeigt die Testergebnisse sehr junger Kinder, die sich im ersten Kindergartenjahr befinden und vom Zeitpunkt der Befragung an noch 11/2 Jahre im Kindergarten verbleiben.

\begin{tabular}{|l|c|c|}
\hline & Alterstufe & Gesamtpunktzahl \\
\hline Ilona & I & 82.5 \\
\hline Florian & II & 82.5 \\
\hline Mark & I & 12.5 \\
\hline Vanessa & II & 10 \\
\hline
\end{tabular}

Tab. 65 Enorme Leistungsdifferenzen bei den ganz jungen Kindern

Sorgen bereiten leistungsstarke Kinder wie Ilona und Florian, die in vielen Kindergärten wohl zuwenig oder überhaupt nicht gefördert werden und ihre Zeit im besten Fall bis zum Schuleintritt vielerorts vor allem damit verbringen, sich sozial und emotional zu entfalten.

Die nächstfolgende Zusammenstellung zeigt die Testergebnisse von Kindern aus dem zweiten Kindergartenjahr, die ein halbes Jahr nach dem Interview in die erste Primarschulklasse ${ }^{60}$ eintreten.

\begin{tabular}{|l|c|c|}
\hline & Alterstufe & Gesamtpunktzahl \\
\hline Phillip & V & 95 \\
\hline Svenja & IV & 87.5 \\
\hline Kilian & V & 37.5 \\
\hline Christine & IV & 20 \\
\hline
\end{tabular}

\footnotetext{
${ }^{60}$ Die ersten vier Jahre der Primarschule in der Schweiz entsprechen der Grundschule in Deutschland. In den meisten Kantonen der Schweiz dauert die Primarschule sechs Jahre.
} 
Tab. 66 Das gleiche Phänomen bei den älteren Kindern

Hier sind beide Leistungsgruppen mit Problemen behaftet: leistungsstarke Kinder wie Phillip und Svenja laufen Gefahr in der künftigen Schulklasse unterfordert zu sein, leistungsschwache Kinder wie Kilian und Christine werden dann wohl überfordert sein.

Bei der Konsultation der Kompetenzskala (Seite 87) wird ersichtlich, dass die leistungsstarken Kinder weit über und die leistungsschwachen Kinder weit unter dem Durchschnitt der entsprechenden Altersgruppen liegen. Auf Grund unserer Untersuchungen kann man davon ausgehen, dass etwa $10 \%$ zu den leistungsstarken und ebenso viele zu den leistungsschwachen Kindern gezählt werden können. Auf jeder Stufe der Alterskala finden sich also Kinder, die aus der Norm fallen und in unseren Institutionen nicht gebührend zur Kenntnis genommen werden.

Ich möchte an dieser Stelle bildlich gesprochen das Fenster öffnen und nicht nur die mathematischen Kompetenzen ansprechen, sondern auch die Lesekompetenz ins Spiel bringen, um zu zeigen, dass die Tatsache der Unterforderung (und Überforderung) nicht nur ein Phänomen der mathematischen Ausbildung darstellt. Eine groß angelegte Längsschnittstudie vom M. Stamm zwischen 1995 und 1998 in der Schweiz zeigt, dass unter 2700 untersuchten Schulanfängerinnen und Schulanfängern 23\% so genannte „Vielkönner“ eruiert wurden, deren Vorsprung gemäß Lehrplan mindestens $1 / 2$ Jahr betrug. 8.1\% waren Frühleser, 6.9\% Frührechner und 8.0\% Frühleser und Frührechner. Aus dieser Gruppe der Vielkönner wurden die sogenannten Alleskönner identifiziert. Das sind solche Kinder, die alle Lese- und/oder Rechentests absolut fehlerfrei absolviert haben. Es handelt sich um knapp 10\% aller 2700 untersuchten Kinder. Diese Kinder wiesen ein Potential auf, das mindestens den fachbezogenen Lernzielen am Ende der ersten Klasse entsprach. M. Stamm drückt sich sehr dezidiert aus, indem sie das Fragezeichen des Titels der Studie „Frühlesen und Frührechnen als soziale Tatsache?“ weggelassen und durch ein Ausrufezeichen ersetzt haben möchte (Stamm in Basellandschaftliche Schulnachrichten 1999, 7).

Das Schicksal der unter- und überforderten Schulkinder wiederholt sich vermutlich jährlich tausendfach in vielen Schulen mit allen daraus resultierenden Konsequenzen für die Kinder, Eltern, Lehrerinnen und Kindergärtnerinnen. Zu dem Problem der kognitiven Heterogenität kommt noch hinzu, dass das Einschulungsalter in der Schweiz und in Deutschland sehr hoch gehalten wird. Das Durchschnittsalter der Einschulung variiert in 
der Schweiz sehr von Kanton zu Kanton ${ }^{61}$, ist aber mit knapp über 7 Jahren sehr hoch (EDK-Dossier 48A 1997); in Deutschland beträgt das Eintrittsalter 6 Jahre und 8 Monate (A. Schavan ${ }^{62}$ in Focus 8/2002, 62). Ilona, Florian und viele andere Kinder müssen also wie schon erwähnt noch $1 \frac{1}{2}$ Jahre bis zum Schuleintritt warten und gehören dann mit hoher Wahrscheinlichkeit zu den Vielkönnern. Kilian, Christine und andere werden eventuell zurückgestellt, weil sie die Schulfähigkeit nicht erreichen ${ }^{63}$. Ein Dilemma der heutigen starren Einschulungspraktiken liegt also auf der Hand: zu viele Kinder könnten wohl, dürfen aber nicht.

Die noch vielfach feststellbaren Berührungsängste zwischen Kindergarten und Schule mit den daraus resultierenden starren Verhaltensnormen ist wohl historisch begründet. Kindergarten und Schule entwickelten sich unabhängig voneinander. Während der Kindergarten aufgrund privater Initiative entstand und lange Zeit eher als Erziehungs- und „Bewahrungsanstalt“ galt, in der die Kinder erzogen und sozialisiert wurden, existiert in der Schweiz die Schule seit ca. 200 Jahren als öffentliche Institution und wird als „Lernort" bezeichnet. Diese unterschiedlichen Traditionen und Lernkulturen, welche Aufgrund der verschiedenen Ausgangslage entstanden, sind ein Grund dafür, dass trotz vielerlei Bemühungen um eine Annäherung ein Graben sichtbar bleibt und der Übertritt der Kinder vom Kindergarten in die Volksschule nicht immer harmonisch, für etliche Kinder sogar problematisch verläuft (EDK Dossier 48A 1997, 5ff).

Die heterogenen Kompetenzdifferenzen sind somit nur ein Aspekt dieser sehr vielschichtigen Problematik des Übergangs Kindergarten - Primarschule. Aber angesichts der gesellschaftlichen Veränderungen, angesichts der Veränderungen namentlich bei den Kindern und deren Eltern und in Anbetracht der Entwicklungen, welche der Kindergarten und die Primarschule in den letzten Jahren genommen haben, scheint eine Diskussion im Bildungsbereich insbesondere der Basisstufe (Verschmelzung von Kindergarten und Primarunterstufe) durchaus angebracht, legitim, nützlich und notwendig.

\footnotetext{
${ }^{61}$ In der Schweiz ist das Schulsystem kantonal geregelt. Es gibt entsprechend den 26 Kantonen 26 verschiedene Schulsysteme, die sich zum Teil sehr stark unterscheiden. Als Beispiel sei nochmals die Primarschuldauer

erwähnt, die im Kanton Basel-Stadt vier Jahre, im Kanton Basel Landschaft fünf Jahre und in der Mehrheit der übrigen Kantone sechs Jahre dauert.

${ }^{62}$ Anette Schavan ist Kultusministerin in Baden-Württemberg und Vorsitzende der Kultusministerkonferenz (Stand Herbst 2002). Sie setzt sich sehr für das Schulprojekt „Schulanfang auf neuen Wegen“ in Baden-Württemberg ein.

${ }^{63}$ Eine Erkenntnis der Pisa-Studie ist aber, dass spät eingeschulte, also zurückgestellte Kinder nicht erfolgreicher sind beim späteren schulischen Lernen.
} 


\subsection{Mathematische Förderung in der Vorschulzeit}

Die Ergebnisse des OTZ beziehungsweise deren Auswertung mit der Vier-Felder-Tafel zeigen an vielen Stellen die notwendigen Voraussetzungen für die Bewältigung von pränumerischen wie numerischen Aufgaben. Oft sind Kompetenzdefizite in beiden Bereichen, aber auch fehlende mentale Modelle dafür verantwortlich, dass Vorschulkinder gewisse Aufgaben nicht lösen können. Diese Defizite können nun mit geeigneten Konzepten und Materialien behoben werden. Es hat sich gezeigt (siehe Seite 185ff), dass sich die verschiedenen Kompetenzbereiche (pränumerisch und numerisch) gegenseitig unterstützen und sich parallel entwickeln. Förderung sollte darum so angelegt sein, dass die „Piaget’schen“ Kompetenzen und Zählkompe-tenzen gleichzeitig berücksichtigt werden.

Werden bei einer mathematischen Frühförderung die kindlichen Anlagen, Möglichkeiten und Neigungen missachtet, können Kinder ihre Neugier und Lernbegierde schon früh verlieren. Die didaktische Arbeit im Kindergarten sollte darum vor allem kindliche Grundbedürfnisse entdecken und nicht die Vermittlung von Wissen in den Vordergrund stellen. Zahlen und Muster sind nicht nur eine Sache des Kopfes, sondern hängen auch eng mit motorischen Erfahrungen und auch mit Emotionen zusammen. Darum sollten bei der mathematischen Frühförderung alle Sinne des Kindes aktiviert werden. Das Montessori- und das Froebel-Material erfüllt in weiten Teilen diese Forderung der ganzheitlichen Förderung. Ich möchte hier aber nicht näher auf dieses Material eingehen.

In letzter Zeit sind Bestrebungen $\mathrm{zu}$ beobachten, diese kindlichen Bedürfnisse nach Spiel, Spaß, Geborgenheit, emotionale Entfaltung, Entdeckungen und selbstständiges Erkunden der Welt auch in der Mathematik unter einen Hut zu bringen. Zu erwähnen ist hier das Kindergartenprogramm „Entdeckungen im Zahlenland“ von G. Preiß (2002, unveröffentlicht). Es geht in der mathematischen Förderung im Kindergarten nie darum, Inhalte und Methoden der ersten Klasse vorzuverlegen, sondern um einen behutsamen Einstieg in die abstrakte und symbolische Welt der Mathematik und die ständige Erprobung der neu gemachten Entdeckungen (siehe auch Einleitung Seite 10). Das von Preiß entwickelte Mathematikprogramm für den Kindergarten versucht Neugier und Freude am Lernen zu wecken. Die bei vielen Erwachsenen feststellbare Angst vor der Mathematik kommt erst gar nicht auf, wenn wie in diesem Programm versucht wird, den Kinder einen spielerischen Zugang ins Zahlenland zu bieten, in dem sich die Kinder dann 
wohlfühlen. Zahlenhaus, Zahlenweg, Zahlenland oder Zahlengarten sind einige Begriffe, die zeigen, dass auf der kindlichen, durchaus auch emotionalen Ebene den Kindern der Einstieg in die Mathematik dargeboten wird. Bei allen diesen Aktivitäten ist immer zu berücksichtigen, dass man die „mathematische Sprache nicht so nebenbei [lernt] wie die Muttersprache“ (ebd). Der Zugang zu dieser Sprache wird in einem für das Kind sinnlich erfahrbaren ,Land' ermöglicht und bei der Entwicklung des Zahlbegriffs kommen verschiedene Aspekte zum Tragen. Der Einstieg und der Zugang in die Welt der Mathematik „öffnet sich den Kindern nur durch organisiertes Lernen“ und dem Entwicklungsstand der Kinder entsprechend müssen sich dabei „die Zahlen den Kindern praktisch und konkret darbieten.“ Es wird darauf geachtet, dass die Kinder die „Welt der Zahlen mit guten Gefühlen“ verbinden und dass die abstrakten mathematischen Inhalte aus Wahrnehmung und Handeln aufgebaut werden und in Anwendungen ständig erprobt werden. Das von G. Preiß entwickelte Programm zum Aufbau des Zahlbegriffs bietet nun wie erwähnt die vier Handlungs- und Erfahrungsfelder Zahlenhaus, Zahlenweg, Zahlenland und Zahlengarten den Kindern an. Im Zahlenhaus hat jede Zahl einen festen Ort, wobei in der , guten Stube' die wichtigen Zahlen von eins bis zehn wohnen. Mit diesen wichtigen Zahlen werden die Kinder im Laufe der Zeit eine intensivere Beziehung aufbauen; sie werden als ,Freunde' betrachtet. Auf dem Zahlenweg nähern sich die die Kinder den Zahlen ,Schritt für Schritt' mit dem wichtigen Werkzeug des Zählens. Als Material dienen Teppichfliesen, auf denen die Zahlen von 1 bis 20 aufgemalt sind, die Fliesen mit den Zahlen 5, 10, 15 und 20 mit einer anderen Farbe. Der Weg kann vorwärts und rückwärts durchlaufen werden und man kann auch an bevorzugten Stellen verweilen. Die Zahlen werden ganzheitlich erlebt und ,zugänglich' gemacht. In den Zahlenländern werden Sinne angeregt, Rätsel gelöst und Ideen entwickelt: Was gibt es nur einmal, welche Dinge treten paarweise auf? Wie viele Beine hat eine Katze und ein Stuhl, wie viele eine Spinne oder ein Vogel? Hier haben die Kinder die Möglichkeit, passende Lieder zu singen oder Abzählreime kennen- und auswendig zu lernen. Schlussendlich wird im Zahlengarten die geometrische Darstellung von Zahlen erschlossen und das ganzheitliche Lernen angestrebt. Der Zahlengarten umschließt die Spiel- und Erkundungswelt des Kindergartens und stiftet so zahlreiche Zusammenhänge zwischen Zahlen, geometrischen Formen und der Natur und fördert so eine differenzierte Wahrnehmung (Preiß 2002, 2ff).

Ein weiteres Programm für mathematische Förderung im Kindergarten ist das „Kleine Zahlenbuch“ (Müller/Wittmann 2002) mit dem ersten Band „Spielen und Zählen“ und 
dem zurzeit noch nicht verfügbaren zweiten Band „Schauen und Zählen“. Die Spiele sollen vor allem die Kinder zur aktiven Auseinandersetzung mit Mathematik anregen, wobei geachtet wurde, dass allen Kinder mit den verschiedensten Kompetenzniveaus miteinander spielen können. Dem sozialen Lernen wird hier ausdrücklich Rechnung getragen. Interessant scheint mir folgender Aspekt: „Die Gegenstände, die zum Zählen und Nachlegen anregen, wurden aus folgendem Grund bewusst nicht nur der Erfahrungswelt der Kinder entnommen: Es ist zwar wichtig, Kinder dort ,abzuholen', wo sie sind. Da sie Dinge aber erst dann wahrnehmen, wenn man sie darauf aufmerksam macht, ist es genauso wichtig, sie dort, hinzuführen', wo sie noch nicht waren“ (Müller/Wittmann 2002, Begleitheft). Es ist dabei immer darauf zu achten, dass einzelne Kinder in ihrer mathematischen Entwicklung nicht deutlich hinter der ihrer Altergenossen zurückbleiben, da solche Lücken im Laufe der Zeit nicht von selbst kleiner, sondern immer größer werden (vgl. Hasemann 2003). Durch den Einsatz des „Osnabrücker Test zur Zahlbegriffsentwicklung“ (OTZ) (siehe Kapitel 6.1, Seite 78) ist man in der Lage zu bestimmen, welche Kinder noch eine spezielle Förderung bedürfen, d.h. bei welchen Kindern die Vorkenntnisse in der Zahlbegriffsentwicklung derart sind, dass sie dem mathematischen Anfangsunterricht in der Grundschule voraussichtlich nicht folgen können. Allenfalls könnten spezielle Förderprogramme eingesetzt werden. Ein solches Programm wird zurzeit in Holland eingesetzt: „Rekenhulp voor kleuters“ (Van Luit \& van de Rijt 1995). Die angebotenen Hilfen für die Kinder zielen dann direkt auf die möglichen Defizite in den acht Komponenten (siehe Seite 79).

Stehen derartige Programme den Kindergärtnerinnen nicht zur Verfügung oder wollen sie neue Aspekte in der frühkindlichen mathematischen Förderung einbringen, so stehen der Kindergärtnerin auch andere Möglichkeiten zur Verfügung. Es geht ja wie schon erwähnt nicht darum, ein bestimmtes Ziel zu erreichen, sondern mathematisches Verständnis zu wecken. Das bedeutet, dass die Kindergärtnerin nicht ein Mehr an Stoff zu behandeln hat, sondern ein bewussterer Umgang mit den einzelnen Situationen ist schon eine sehr gute Basis für mathematisches Tun. Sie erkennen dann, dass vieles, was sie bisher mit ihren Kindern schon spielten oder bearbeiteten, mathematische Vorerfahrungen sind, ohne dass sie dies bis anhin bewusst wahrgenommen hätten. Aus dieser Optik stellt man fest, dass Mathematik in fast allen Bereichen des Kindergartenalltages anzutreffen ist. Dies kann sich die Kindergärtnerin zu Nutze machen und - ohne ein eigenes Fach Mathematik zu führen - die Kinder spielerisch mit folgenden Inhalten mathematisch fördern. Dabei geht es insbesondere um: 
- das Vergleichen, Klassifizieren und Ordnen von Objekten

- das Erfassen in die Invarianz von Größen und von Mengen

- das Herstellen von Eins-zu-eins-Zuordnungen

- den Gebrauch von Zahlwörtern und -zeichen sowie um die verschiedenen Aspekte beim Ab- und Auszählen

- Erfahrungen mit geometrischen Grundbegriffen und Objekten

- das Erkennen und Herstellen von Figuren und Mustern

- Beschreiben von Sachverhalten, Gemeinsamkeiten, Unterschieden und um den Aufbau von mentalen Bildern

( siehe Hasemann 2003, 39).

Ich gebe im Folgenden einige Beispiele, wie mathematische Förderung in einem Kindergarten aussehen könnte (siehe auch Koch 2000, 22-24):

Mit einem Form-, Farb- oder Ziffernwürfel erwürfeln die Kinder beim Schlangenspiel eine beliebig lange Schlange, dessen Teile aus geometrischen Grundformen in verschiedenen Farben entsprechend den Würfelfarben bestehen. Dadurch können z.B. folgende mathematische Vorerfahrungen gemacht werden:

- Formerfassung

- Farbkenntnis

- Seriation

- Zahlbegriff, etc.

Geburtstagskalender können durch bewusste Gestaltung mathematische Vorerfahrungen bieten. Die Kinder können ihre eigenen Symbole erkennen. Sie sehen z.B., in welcher Jahreszeit sie Geburtstag haben, in welcher Reihenfolge die Geburtstage stattfinden und wie alt sie sind. Weitere Vorerfahrungen könnten sein:

- Zeitbegriffe lang-kurz,

$$
\text { früher - später }
$$

- Seriation Jahreszeiten

- Zahlbegriff Kardinal-, Ordinal- und Maßzahl

- Vergleichen Gleich alt, älter, jünger (Für Kindergartenkinder schwer zu verstehen: Wer später geboren wurde ist jünger!) 
Im Turnunterricht sind viele originale Begegnungen mit der Mathematik in Spiel und Sport möglich. Nur einige mathematische Vorerfahrungen seinen erwähnt:

- Raumerfahrung Beim Herumrennen und -toben in der Turnhalle.

- Seriation Stafette; Einer nach dem andern.

- Ordinalzahl Du bist zweiter, ich bin der Vierte.

- Vergleiche Weiter, höher, schneller, usw.

- Maßzahlen Weiten in Meter (und Zentimeter), Dauer in Minuten (und Sekunden), usw.

etc.

Viel Aktivitäten im Fach Rhythmik können und sollen für eine umfassende Raumerfahrung genutzt werden. Oben/unten, hinten/vorne sind nur eine Auswahl dieses Aspektes. Wie im Kapitel 9 festgestellt, nutzen Kinder rhythmische Bewegungen bei Zählprozessen und da kann man fördernd mit den vielfältigen Möglichkeiten des Faches Rhythmik unterstützend eingreifen.

Sehr umfangreich ist Mittlerweilen die Auswahl an Kinderbücher, die mathematische Themen zum Inhalt haben. Gelungene und weniger gelungene Beispiele gibt es Zuhauf. Als gute Exempel von themenbezogenen Kinderbüchern seien genannt: „zwei und mehr“ (Fuchshuber 1997), das die Kardinalzahl zum Thema hat und „fünfter sein“ (Jand1/Junge 2001), das die Ordinalzahl in den Vordergrund stellt. Das Kinderbuch im Kindergarten muss aber nicht Mathematik thematisieren, um trotzdem ein Fülle von mathematischen Themen zu liefern. Das Beispiel „Die dumme Augustine“ (Preussler/Lenz) möge dies illustrieren. Die Kindergärtnerin kann ihren Morgen wie gewöhnlich aufbauen: spannend erzählen, die Aufmerksamkeit der Kinder auf feine Einzelheiten lenken und sie spielend, bastelnd, singend und tanzend erleben lassen, was im Buch dargestellt ist. Die Muße, sich rückblickend einzelnen stark wirkenden Bildern und Episoden zuzuwenden und tiefer in sie einzudringen, stellt sich dann von selber ein. An diesem Beispiel „Die dummen Augustine“ wird deutlich, dass viele mathematische Elemente im Umfeld der Kinder vorhanden sind. Sie müssen nur noch wahrgenommen werden. Dies ist eine der Aufgaben der Kindergärtnerin. So könnten mit diesem Buch die folgenden mathematischen Inhalte thematisieren werden (Koch 2000, 22):

Zeitbegriffe

S. 6/7 Wie oft am Tage findet eine Zirkusvorstellung statt? 
S. 8/9 Tagesablauf

S. $10 / 11$ Nacht

S. 14/15 ev. Uhrzeit - Vergleich mit der Zeitspanne eines Kindergartenvormittags

S. 25 Abend

Muster und Reihenfolgen

Im ganzen Buch immer wieder anzutreffen, vor allem aber:

S. 4/5 Gemusterte Drachen, Pferdedecken, Elefantendecken

S. 6/7 Reihenfolge der Balancierutensilien (Aufstapeln)

S. 10/11 Strukturierte Kopfkissenbezüge

S. 2/3 - S. 10/11 Größer - kleiner als, am größten - am kleinsten

S. 16/17 Manege

S. 6/7 - S. 22/23 Ablauf, resp. Reihenfolge der Vorführung

Formerfassung

S. 1/2 Reifen, Ballon: rund

S. 5 Drachen: Viereck

S. 6 Balancierutensilien: Trapez, Kugel, Stab, ...

S. 8/9 Backformen: Herz, Stern, Rad

S. 10/11 Mond: sichelförmig; Bilderrahmen: rechteckig, Lampenformen:trapezförmig

S. 17 Pfeil

S. 22 zwei verschiedene Sternenformen

\section{Raumerfahrung}

Am Anfang und am Ende des Buches ist eine Spiegelung abgebildet (Schuhe mit Mäusen).

S. 2/3 - S. 16/17 Zirkuszelt

S. 16/17 Manege

Masse

S. 8 Zusammen backen: Hohlmasse gebrauchen

S. 9 Tintenfässchen, Teller, Schüsseln, Schöpfkellen, Kessel

S. 14 Spritze

Zählen

S. 2/3 Familienmitglieder, Haustiere

S. 4/5 Tiere, Drachenschwanz, Wagennummer 
S. 8/9 Anzahl der ausgestochenen Plätzchen

S. 10/11 Mäuse, Betten - Personen

S. 12/13 Tränen, Schwanzfedern des Papageis

S. 16/17 Tiere, Zuschauer, Artisten, ...

(Aus Hausarbeit C. Brodmann, 1999)

Geeignete Lieder, Fingerverse (bis Zahl 5, dann bis 10) und Gedichtchen „Morgens früh um 6 kommt die alte Hex ...“ ebenso wie Abzählverse „1, 2, 3, 4, 5, 6, 7 wo isch ächt der .... bliibe?" sind für die Steigerung der Sicherheit in Zeitabläufen wie auch für die Erschließung des Zahlenraum überaus wichtig. Vorwärts- und Rückwärtszählen können mit Kinderversen und Abzählreimen automatisiert und gefestigt werden. Ein Beispiel zum Rückwärtszählen möge das illustrieren (Janosch 1984, 151):

\author{
Zehn Gänse im Haberstroh, \\ die saßen da und waren froh. \\ Da kam der Fuchs gegangen \\ Und wollte eine fangen \\ Und sagte: So! \\ Neun Gänse im Haberstroh ... \\ Acht ... \\ Sieben ... \\ Sechs ... \\ Fünf ... \\ Vier ... \\ Drei ... \\ Zwei ... \\ Eine Gans im Haberstroh, \\ die saß da und war froh. \\ Da kam der Fuchs gegangen \\ Und wollte eine fangen \\ Und sagte: So!
}

Am Beispiel des Tischdeckens und des Essens und Trinkens bei einer Geburtstagsfeier wird nochmals deutlich, wie vielfältige mathematische Erfahrungen gemacht werden können, wenn man sie nur zur Kenntnis nimmt. Kindergerecht aufbereitet kann so ge- 
zeigt werden, dass Mathematik und Alltag sehr oft zusammengehören (siehe Schmassmann 1986, 17).

Farbe Grundfarben, Musterfarben, Farbtöne, Farbkontraste, glänzend matt, einfärbig - bunt ...

Form Ist der Tisch rechteckig, quadratisch oder rund? Die Tischdecke hat die gleiche oder eine andere Form, nur ist sie größer - sie hängt über den Rand. Teller haben einen runden Boden. Es gibt tiefe und flache Teller. Die Schüssel hat auch einen runden Boden, aber die Seitenwand ist höher (von außen gesehen) oder der Grund liegt tiefer (nach innen betrachtet). Trinkbecher haben ebenfalls einen runden Boden, aber die Seitenwand ist noch höher als bei einer Schüssel.

Das Besteck kann schmal, länglich, oval, gebogen, ... sein.

Die Serviette ist quadratisch, wird sie gefaltet, entsteht ein Dreieck.

Die Speisen haben unterschiedliches Aussehen und verschiedene Formen, die sich während des Essens auch ändern.

Dimension

klein - groß, flach - tief, ... (z.B. Geschirr)

lang - kurz, dünn - dick, ... (z.B. Besteck)

Volumen: Gefäße füllen oder leeren (einschenken, austrinken),

Höhe der Flüssigkeit im Becher, weniger - mehr, ...

Materialeigenschaften rau - glatt, weich - hart, matt - glänzend, kalt - warm, nass trocken.

Gewicht leicht - schwer

Zeit

Vormittag - Pause; Mittag - Mittagessen; Spielzeit - Puppenküche

Zeitdauer

Je mehr Kinder beim Auf/Abdecken helfen, desto weniger Zeit brauchen wir.

Reihenfolge zuerst, dann, vorher, nachher.

Tischtuch - Teller - Becher - Besteck - Serviette - Kanne - Essen - Tisch abräumen - sauber machen - neu decken ...

Zuordnung und Tisch - Tischtuch - Teller - Besteck - Serviette - Becher ...

Anordnung für jeden ein Platz - mein Platz - dein Platz

Teller - Essen, Becher - Getränk, Suppe - Löffel, feste Speisen 
- Messer und Gabel, Abfälle - Mistkübel ...

Am Puppentisch: Ich bin die Mutter - du bist Vater - du das Kind ...

Mengen durch

Teller, Tassen, Becher, Schüssel: Geschirr

Oberbegriffe

Saft, Tee, Milch, Kakao: Getränk

Kartoffel, Karotten, Salat: Gemüse

etc.

Teilen und

Vervielfachen

Relationen

Ordnungsrelationen

Zählen

Raumlage und

räumliche Beziehungen gegenüber, umgekehrt ... (Wo liegt das Besteck auf dem Platz gegenüber?)

Muster

Symmetrie

Beim Tischdecken gehe ich dreimal zum Tisch und bringe je zwei

Teller. Aufteilen des Essens/Trinkens: Jedes Kind bekommt einen Teil. Ein großes Stück wird in ein kleineres zerteilt, viele kleine Stücke können gleich viel sein wie wenige große (Substanzerhaltung).

Obst wird in gleich große Teile geteilt (z.B. Viertel); Geschirr zerbricht. Aus einem Ganzen werden Teile (z.B. drei Viertel). Ist der ausgeschüttete Saft auf dem Tisch gleich viel wie vorher im Becher? Ist im schmalen hohen Glas mehr Saft als im niedrigen breiten?

Größer - kleiner, Schmeckt gut - besser, näher beim Teller weiter weg, mehr - weniger essen/trinken ...

die erste, die zweite, ... Gruppe; nach mir, vor mir, ... 12 Plätze, 12 Gedecke ...; ich esse eine Portion oder vielleicht doch noch eine zweite, ...

Wenn jedes Kind eine Portion isst, essen alle zusammen 12 Portionen.

oben - unten, rechts - links, außen - innen, darunter - darüber

Die Tischdecke ist mit Muster verziert; ebenso ist der Geburtstagskuchen garniert mit Smarties, Gummibärchen, Schokoladenherzchen.

Tischdecke zusammenlegen, Serviette falten ... 
Motorik

Sensorik
Tischdecke zusammenfalten, Aufteilen der Gedecke, Halten des Bechers ..., Gedecke einsammeln, wegtragen; Tisch aufräumen und abwischen.

Aufwischen des ausgeleerten Saftes: Das Kind spürt die Nässe, die Struktur des Tuches, des Tisches ...

Brösel fühlen sich spitz, hart an ...

Es ist selbstverständlich, dass aus dieser Auswahl nicht alles auf einmal ausgeschöpft werden darf. Die Kindergärtnerin setzt die Akzente entsprechend dem zurzeit aktuellen Thema.

Zum Abschluss möchte ich noch an einem konkreten Beispiel darstellen, wie eine mathematische Förderung in einem Kindergarten aussehen kann. Die Kindergärtnerin Rosina Tschopp zeigt dies auf eindrückliche Art und Weise am Beispiel einer Werkstatt zum Thema Ostern. Frau Tschopp hat diese Werkstatt selbständig entwickelt und lässt die Kinder weitgehend selbstständig in Eigenverantwortung an den einzelnen Posten arbeiten. Vor einem Einsatz der Werkstatt werden zudem die Eltern der Kinder umfassend über diese mathematische Förderung informiert, unter anderem wird die Werkstatt interessierten Eltern vorgestellt. Im Allgemeinen sind Eltern positiv gegenüber solcher mathematischer Förderung eingestellt. Alle Kinder erhalten einen Werkstattpass, auf dem ihre Aktivitäten festgehalten werden und die Kinder zeigen diesen Pass immer wieder den Eltern, vorauf die Eltern auch mal Kommentare und Fragen aufschreiben können. Auch auf diese Weise entwickelt sich eine Kommunikation zwischen Eltern und Kindergärtnerin und zudem sind die Eltern über die mathematischen Aktivitäten der Kinder stets informiert und auf dem Laufenden.

Frau Rosina Tschopp legt Wert auf eine ganzheitliche Förderung der Kinder und vor allem auf das soziale Lernen. Der Verfasser hat im Frühjahr 2002 mit eigenen Augen verfolgen dürfen, wie die Kinder mit Engagement und mit Freude und Lust an den einzelnen Werkstattposten gearbeitet haben. Oft konnte beobachtet werden, wie sich die Kinder gegenseitig halfen und unterstützten. Kein Kind hatte Scheu vor einer Aufgabe und sie lösten so gut es ihren individuellen Kompetenzen entsprach die entsprechenden Aufgaben. Es gab nie eine Situation, in der ein Kind frustriert und entmutigt von einer Aufgabe ließ. Als Gesamteindruck erweckte die Schar der Kinder einen fröhlichen und entspannten, aber immer konzentrierten Eindruck bei der Bearbeitung dieser Werkstatt 
zum Thema Ostern. Frau Tschopp hat mir freundlicherweise ihre Werkstatt zur Ansicht zur Verfügung gestellt.

$\mathrm{Zu}$ den einzelnen Werkstattposten:

\begin{tabular}{|c|c|c|}
\hline Posten/Material & Beschreibung & Ziel \\
\hline $\begin{array}{l}\text { Eierwanne } \\
\text { - Verschiedene Eierpaare } \\
\text { mit unterschiedlicher } \\
\text { Oberflächenstruktur } \\
\text { - Spreu } \\
\text { - Wanne, Kiste }\end{array}$ & $\begin{array}{l}\text { - Die Eier sind in der Wanne im } \\
\text { Spreu versteckt. Ein Kind zieht ein } \\
\text { Ei aus dem Spreu und dann das } \\
\text { zweit dazu passende usw. } \\
\text { - Kann auch als Partnerarbeit ge- } \\
\text { spielt werden, indem das erste } \\
\text { Kind ein Ei zieht und das zweite } \\
\text { Kind ertastet sich das dazu passen- } \\
\text { de, usw. }\end{array}$ & $\begin{array}{l}\text { Die Kinder ordnen taktil } \\
\text { gleiche Oberflächenstruk- } \\
\text { turen zu (Differenziertes } \\
\text { Wahrnehmungsvermö- } \\
\text { gen, siehe Seite 209). }\end{array}$ \\
\hline $\begin{array}{l}\text { Arbeitsblätter } \\
\text { - Bild mit Würfeln } \\
\text { - Bild mit Ziffern }\end{array}$ & $\begin{array}{l}\text { Die Kinder malen das Bild nach } \\
\text { Zahlen aus. Jede Zahl steht für eine } \\
\text { Farbe. }\end{array}$ & $\begin{array}{l}\text { Die Kinder erkennen eine } \\
\text { bestimmte Menge. Die } \\
\text { Kinder kennen die Ziffern } \\
\text { und können sie zuordnen } \\
\text { (Formerkennung und } \\
\text { Zählkompetenz, siehe } \\
\text { Seite 209). }\end{array}$ \\
\hline $\begin{array}{l}\text { Eierfabrik } \\
\text { - Knetmasse } \\
\text { - Eierkärtchen mit ver- } \\
\text { schieden vielen Eiern } \\
\text { daraufgemalt. } \\
\text { - Zifferkärtchen mit Zif- } \\
\text { fern daraufgemalt. }\end{array}$ & $\begin{array}{l}\text { - Die Kinder suchen sich fünf ver- } \\
\text { schiedenen Eierkarten aus. Zu je- } \\
\text { der Karte formen sie die passende } \\
\text { Anzahl Eier aus Knet. Am Schluss } \\
\text { legen sie die Karte mit den Ziffern } \\
\text { noch dazu. } \\
\text { - Die Kinder suchen sich zwei Eier- } \\
\text { kärtchen und formen die Summe } \\
\text { der darauf abgebildeten Eier aus } \\
\text { Knet. } \\
\text { - Die Kinder wählen Ziffernkärtchen } \\
\text { und formen die entsprechende An- }\end{array}$ & $\begin{array}{l}\text { - Die Kinder lernen die } \\
\text { Mächtigkeit einer Men- } \\
\text { ge erkennen. } \\
\text { - Sie können zu einer } \\
\text { bestimmten Anzahl die } \\
\text { Ziffern hinlegen. } \\
\text { - Addition kann enaktiv } \\
\text { erfahren werden. } \\
\text { - Eins-zu-eins- } \\
\text { Zuordnung Ziffer-Zahl- } \\
\text { Menge (Zählkompe- } \\
\text { tenz, Mustererkennung, }\end{array}$ \\
\hline
\end{tabular}




\begin{tabular}{|c|c|c|}
\hline & zahl Eier aus Knet. & siehe Seite 209). \\
\hline \begin{tabular}{|l} 
Würfelkästchen \\
- Würfelkästchen (Zau- \\
berkasten) \\
- Mustervorlage
\end{tabular} & $\begin{array}{l}\text { Das Kind ordnet die Würfel im Käst- } \\
\text { chen so an, dass das vorgegebene } \\
\text { Muster entsteht }\end{array}$ & $\begin{array}{l}\text { Die Kinder üben sich im } \\
\text { Formerfassen und der } \\
\text { Raumorientierung (siehe } \\
\text { Seite 209). }\end{array}$ \\
\hline $\begin{array}{l}\text { Muster-Eier } \\
\text { - Muster-Eier } \\
\text { - Legematerial }\end{array}$ & $\begin{array}{l}\text { - Das Kind legt auf dem Ei das vor- } \\
\text { gegebene Muster nach und führt es } \\
\text { weiter. } \\
\text { - Das Kind erfindet selber Muster } \\
\text { nach einem eindeutigen Bildungs- } \\
\text { gesetz. }\end{array}$ & \begin{tabular}{|l} 
- Die Kinder lernen ein \\
Muster zu erkennen und \\
es weiterzuführen. \\
- Seriation \\
(Mustererkennung, \\
schnelle Analyse von \\
Problemstellungen, sie- \\
he Seite 209).
\end{tabular} \\
\hline \begin{tabular}{|l} 
Eierpuzzle \\
- Verschieden schwere \\
Eierpuzzle
\end{tabular} & $\begin{array}{l}\text { Die Kinder führen die verschiedenen } \\
\text { Eierpuzzles zusammen. }\end{array}$ & $\begin{array}{l}\text { Formerfassung üben (sie- } \\
\text { he Seite 209). }\end{array}$ \\
\hline $\begin{array}{l}\text { Eierzählen } \\
\text { - Papier-Eier } \\
\text { - Spielbrett (A3 Papier mit } \\
\text { aufgeklärten Eiern) }\end{array}$ & $\begin{array}{l}\text { - Die Kinder legen die Eier so hin, } \\
\text { dass auf beiden Seiten gleich viele } \\
\text { Eier liegen. } \\
\text { - Fehlende ergänzen } \\
\text { - Zählen und nachlegen } \\
\text { - Zusammenzählen und hinlegen }\end{array}$ & $\begin{array}{l}\text { Die Kinder üben zu zäh- } \\
\text { len (siehe Seite 209). }\end{array}$ \\
\hline $\begin{array}{l}\text { Cominos } \\
\text { - Combino } 1 \\
\text { - Combino } 2\end{array}$ & $\begin{array}{l}\text { Die Kinder lösen die Aufgaben von } \\
\text { einfach bis schwer. }\end{array}$ & $\begin{array}{l}\text { Formerfassung üben } \\
\text { (siehe Seite 209). }\end{array}$ \\
\hline $\begin{array}{l}\text { Würfel } \\
\text { - Großer Würfel }\end{array}$ & $\begin{array}{l}\text { - Ein Kind würfelt und ruft ein ande- } \\
\text { res auf. Dieses Kind hüpft so oft } \\
\text { wie der Würfel anzeigt. } \\
\text { - Es wird zweimal gewürfelt und es } \\
\text { wird so oft gehüpft, wie die die } \\
\text { Summe der gewürfelten Augenzah- } \\
\text { len anzeigt. }\end{array}$ & $\begin{array}{l}\text { - } \text { Das Zählen wird geübt. } \\
\text { - Addition } \\
\text { (siehe Seite 209). }\end{array}$ \\
\hline
\end{tabular}




\begin{tabular}{|l|l|l|}
\hline Ketten & Die Kinder reihen auf der Schnur & Mustererkennung (siehe \\
- Schnur & Perlen nach einem selbstgewählten & Seite 209). \\
- Perlen & eindeutigen Muster auf. & \\
\hline
\end{tabular}

\subsection{Die Basisstufe}

Der ganze Themenkreis hat dazu geführt, dass z.B. in Holland wie bereits erwähnt bereits vor ca. 20 Jahren und in letzter Zeit auch in der Schweiz ein Umdenken stattgefunden hat und entsprechende Schritte unternommen wurden. Die starre Trennung Kindergarten - Primarschule wird vielerorts fallen gelassen und durch ein Modell des jahrgangsübergreifenden Anfangsunterrichts für vier- bis achtjährige Kinder ersetzt. Dabei ist die Lerngruppe, die sich nicht am Alter der Kinder, sondern am Lernstand der Kinder orientiert, die zentrale Einheit, wobei diese Einheiten für alle Kinder gemäß ihren momentanen Kompetenzen offen sind. Dadurch wird eine frühe optimale Förderung und Anregung gewährleistet, da die Kinder ihren Neigungen und Kompetenzen entsprechend gezielt die ihnen zusagenden Lerngruppen besuchen können. Dabei wird einerseits kostbare Lernzeit nicht vergeudet und andererseits wird den Kindern zugestanden, sich individuell zu entwickeln und ihrem geistigen Potential auch genügend Zeit zu lassen. Die Basisstufe ermöglicht die Einschulung der Kinder entsprechend ihrem individuellen Entwicklungsstand und verbessert die frühe spezifische Förderung der einzelnen Kinder im Rahmen des regulären Unterrichts. In Abhängigkeit ihrer besonderen Begabungen und speziellen Lernvoraussetzungen wird in Verbindung der variablen Durchlaufzeiten ein enormes Potential der individuellen Förderung ermöglicht, insbesondere dadurch, dass die Kinder weder überfordert noch unterfordert sind. Diese in der Pädagogik nicht neuen Ideen der jahrgangsheterogenen Klassen und der Individualisierung in heterogenen Gruppen mit Stufen-Lernzielen erlebt durch die veränderten Umstände nur eine Wiederbelebung. Durch das Arbeiten in altersdurchmischten Gruppen wird die Effizienz des Lernens gefördert, wenn das Voneinanderlernen zwischen Kindern, die Eigenaktivitäten der Schülerinnen und Schüler und die Übernahme von zunehmender Verantwortung für den eigenen Lernprozess umgesetzt werden können. Auch die Leitideen der Gemeinschaftsbildung und Integration eröffnen wertvolle pädagogische Perspektiven: Die Förderung der Erziehung zur Teamfähigkeit, zum Erbringen von Leistungen in und für eine Gemeinschaft; die Aufhebung 
der Vereinzelung resp. Vereinsamung von Einzelkindern mit wenig Spiel-Nachbarn (langer Verbleib in einer altersheterogenen Gruppe); der Ersatz des falschen, unfairen Wettbewerbs unter Gleichaltrigen durch die Anerkennung der Verschiedenheit der Kinder einer Gruppe als Grundprinzip der Klassenbildung und als Chance der produktiven Nutzung. Es lässt sich aus Erfahrungen in Mehrklassenschulen belegen, dass die Integration von Kindern mit unterschiedlichem kulturellen Hintergrund in solchen heterogenen Gruppen besser gelingt und damit zum Beispiel Disziplinschwierigkeiten und Gewalterscheinungen unter Kindern stark reduziert werden können oder gar ganz verschwinden ${ }^{64}$. Es ist bei so einem beschriebenen Konzept jedoch unbedingt dafür zu sorgen, dass diese Bildungsanliegen auch in den nachfolgenden Schulstufen konsequent weitergeführt werden. Würde man die weiterführenden Klassen nach der Basisstufe nach diesem Konzept handhaben, d.h. nicht das Alter, sondern die Leistungsfähigkeit wäre maßgebend für die Einreihung in die für das Kind ideale Lerngruppe, so könnte wohl von der umfassendsten Bildungsreform aller Zeiten gesprochen werden.

Da jedes Kind sein spezielles Förderungsbedürfnis hat und mit Stärken und Schwächen ausgestattet ist, verdient es besondere Aufmerksamkeit. In einer jahrgangsheterogenen, individualisierenden und mit variablen Lernzeiten arbeitenden Gruppe verringert sich der Leerlauf im Unterricht, reduzieren sich Störungen und es gibt kein klassisches Sitzen bleiben mehr. Es erhöhen sich die Erfolgserlebnisse und damit die Motivation der Kinder. Eine Konsequenz und in der Logik der jahrgangsgemischten Klassen und der individuellen Förderung ist die Idee des halbjährlichen Eintrittes in die Basisstufe. Die Schuleintritte während des laufenden Schuljahres sind übrigens in Gebieten mit hoher Mobilität der Bevölkerung schon längst Tatsache.

In weiten Kreisen der Bevölkerung und auch bei Politikerinnen und Politikern ist die Meinung verbreitet, dass man die „Kinder vor allzu frühem Leistungsdruck“ (Parlamentsdabatte des Kantons Zürich zur Einführung der Grund- bzw. Basisstufe vom 28.5.2002) bewahren solle. Die Gegner der Basisstufe forderten auch ,zwei unbeschwerte Jahre im Kindergarten“ (ebd.). Immerhin hat man in dieser Parlamentssitzung das bis dahin geltende Lese-, Schreib- und Rechenverbot in den Kindergärten des Kantons Zürich aufgeho-

\footnotetext{
${ }^{64}$ Der Verfasser kann aus eigener Erfahrung von einer alterheterogenen Gruppe sprechen: Er hat ein halbes Jahr in einem kleinen Bergdorf in Graubünden die 20 Schülerinnen und Schüler der ersten sechs Primarschulklassen in einer Gesamtschule unterrichtet. Diese altersheterogene Gruppe war ein außerordentlich gut funktionierender Mikrokosmos und alle (inkl. Lehrer) haben voneinander im höchsten Masse profitiert. Alle waren sozial eingebettet, man hat sich gegenseitig geholfen und unterstützt und im Weiteren waren die von den Kindern erbrachten Leistungen durchwegs gut bis sehr gut.
} 
ben. „Man raubt den Kindern die Kindheit.“ oder „Gönnt dem Kind doch noch ein Jahr!“ sind oft gehörte Einwände; der Schonraum des Kindergartens wird als heilig empfunden. Eine breit angelegte Untersuchung bei Kindergärtnerinnen im Kanton Bern belegt nun aber, dass keine Ablehnung dieser Expertinnen gegenüber einer kognitiven Förderung der Kinder schon ab vier Jahren besteht, im Gegenteil. Die Untersuchung belegt die Offenheit dieser Kindergärtnerinnen bezüglich neuer Strukturen und Inhalten in der Bildung von vier- bis achtjährigen Kindern und es bestehen seitens der befragten Kindergärtnerinnen keine Bedenken gegen eine zu frühe „Verschulung“ der Kinder (Grob 2001).

Selbstredend müssen bei der Bildung einer Basisstufe die notwendigen Rahmenbedingungen geschaffen werden. $\mathrm{Zu}$ denken ist da namentlich an eine angemessene Aufstockung von Stellenprozenten pro Klasse, eine gründliche Ausbildung der Lehrkräfte zur Binnendifferenzierung, die Zuerkennung tieferer Stundenzahlen und Klassengrößen und die Vermeidung jeglicher prestige- und besoldungsmäßiger Schlechterstellung der Lehrkräfte dieser Stufe (vgl. Strittmatter 1998). Der Status der Kindergärtnerinnen in Deutschland liegt im Argen, was sich unter anderem immer auch im Gehalt bemerkbar macht. Die Kindergärtnerinnen resp. Erzieherinnen verdienen bestenfalls zwei Drittel des Gehalts der Grundschullehrerinnen. Die Ausbildung ist in Deutschland noch an Fachschulen angesiedelt und bildet mit Österreich zusammen das Schlusslicht der europäischen Länder. In den Beruf der Kindergärtnerin lenkt man nach wie vor Frauen, die keine guten Erfahrung mit dem Lernen gemacht haben und wohl größtenteils nicht sehr empfänglich für Innovationen sind. D. Elschenbroich beklagt in ihrem Buch „Weltwissen der Siebenjährigen“ dann auch, dass die elementarpädagogische Szene in Deutschland vor allem sich selbst kennt und - von wenigen Ausnahmen abgesehen - von allen europäischen Anregungen abgeschnitten ist (vgl. Elschenbroich 2002, 17ff). Die Situation in der Schweiz sieht glücklicherweise ein wenig anders aus. Die Kindergärtnerinnen haben in den letzten Jahren in vielen Kantonen den Status von Lehrerinnen erreicht und die Ausbildung ist sehr sorgfältig und umfassend. Erwähnen möchte ich die Ausbildung der Kindergarten/Unterstufenlehrkräfte an der Pädagogischen Hochschule beider Basel in Liestal. Diese Lehrerinnen und Lehrer erhalten die Lehrbefähigung für den Kindergarten sowie für die Primarunterstufe. Diese Lehrkräfte sind prädestiniert für den Einsatz in der Basisstufe. Um zu dokumentieren, dass die oben zusammengetragenen Gedanken durchaus Aussicht auf Verwirklichung haben, sei kurz der aktuelle Stand in Deutschland und der Schweiz erwähnt. In Baden-Württemberg läuft seit 1997 der heute vermutlich größte Schulversuch Deutschlands. In den Mittlerweilen 657 teilnehmenden Grundschulen 
ist der Schuleintritt zweimal im Jahr, im Frühjahr und im Herbst, möglich. Wenn ein Kind will, kann es schon mit fünf Jahren mit Lernen beginnen. Dieser „Schulanfang auf neuen Wegen“"gewährleistet in altersgemischten Klassen eine für jedes Kind angemessene Förderung. Die Zurückstellungsquote hat sich denn auch in BadenWürttemberg in den letzten acht Jahren von $10.4 \%$ auf $6.2 \%$ reduziert und die Zahl der vorzeitigen Einschulungen hat sich von $1.4 \%$ auf $8.4 \%$ gesteigert (J. Otto in: Die Zeit 37/2002).

Ab August 2003 starten in den Kantonen Aargau, Thurgau, St. Gallen und Appenzell Ausserrhoden Versuchsklassen. Im Kanton Zürich befand das Volk im November 2002 mittels einer Volksabstimmung über die Einführung eines neuen Einschulungskonzeptes. In allen Kantonen geht es zum einen um die so genannte Grundstufe $^{65}$. Diese Grundstufe umfasst die bisherigen beiden Kindergartenjahre und das erste Primarschuljahr. Wie schon erwähnt (siehe Grob 2001), wird dieser fundamentale Wechsel der Einschulungspraxis auf der ganzen Linie von den Expertinnen resp. Kindergärtnerinnen getragen und unterstützt ${ }^{66}$. Die Einzuschulenden können dann je nach individuellem Können und Leistungsvermögen den Stoff dieser Stufen innert zwei bis vier Jahren erarbeiten und dann in die zweite Primarklasse einsteigen $^{67}$. Zum andern geht es um die Basisstufe, die im Kanton Zürich nicht zur Debatte stand, in den übrigen erwähnten Kantonen aber neben der erwähnten Grundstufe parallel als Versuchsklassen geführt werden. Bei der Basisstufe handelt es sich um die im Text vorgestellte und propagierte Stufe der vier- bis achtjährigen Kinder. Darin eingeschlossen sind die ersten zwei Kindergartenjahre sowie die ersten zwei Jahre der Primarschule. Der Übertritt in die dritte Primarschulklasse erfolgt je nach individuellen Voraussetzungen nach drei bis fünf Jahren. Unterrichtet wird an der Basisstufe

\footnotetext{
${ }^{65}$ Nicht zu verwechseln mit der Grundschule in Deutschland.

${ }^{66}$ Die Organisation der Kindergärtnerinnen und der Kindergartenbehörden des Kantons Zürich haben für ein Ja bei der Volksabstimmung im November 2002 geworben. Im Wesentlichen führten sie vier Gründe für diesen Schritt an:

Erstens könne damit die Chancengleichheit gewährleistet werden, da es derzeit sehr unterschiedliche Kindergärten in den Gemeinden gebe. Zweitens werde die künstliche Trennung zwischen Spielen und Lernen aufgehoben, weil die Kinder fliessend zum Rechnen und Lesen übergehen und zwischendurch auch wieder spielen könnten. Und drittens erlaube es die Grundstufe, den Missstand zu beheben, dass heute ca. 20\% der Kinder entweder zu früh oder zu spät eingeschult würden. In der Grundstufe werden alle Kinder aufgenommen und die Kinder werden weitergegeben, wenn der richtige Zeitpunkt dazu gegeben sei. Viertens, so die Kindergärtnerinnen, erlaube es die Grundstufe den wachsenden Bedarf an unterschiedlicher Betreuung gerecht zu werden und zudem könne auf neue Bedürfnisse besser eingegangen werden (Neue Zürcher Zeitung vom 29.10.2002).

${ }^{67}$ Das Stimmvolk des Kantons Zürich hat die umfassende Einführung des neuen Einschulungskonzeptes Ende November 2002 vorerst abgelehnt und willigte nur einer Versuchsphase zu diesem Konzept zu.
} 
(und der Grundstufe) im Teamteaching von je einer Kindergärtnerin und einer Primarlehrerin zu einem 150\%-Pensum. Dieses neue System bedeutet einen endgültigen Abschied von der Jahrgangsklasse und der Aargauer Bildungsdirektor spricht denn auch von einem „Kulturwandel im Schulwesen“ (R. Huber in: Neue Luzerner Zeitung vom 3.10.2002, Nr. 228). 


\section{Abbildungsverzeichnis}

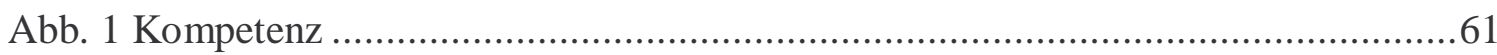

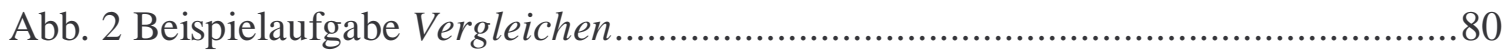

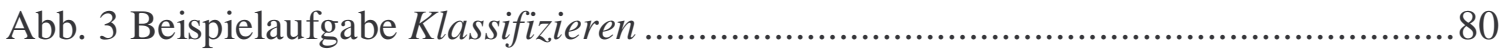

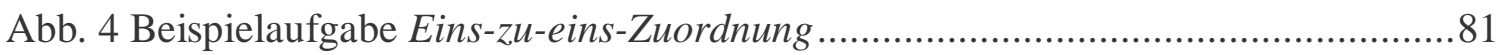

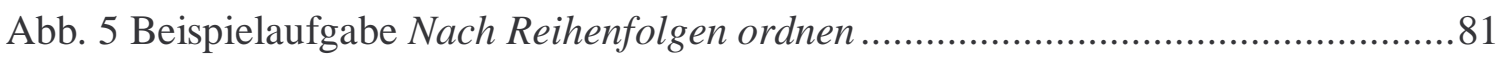

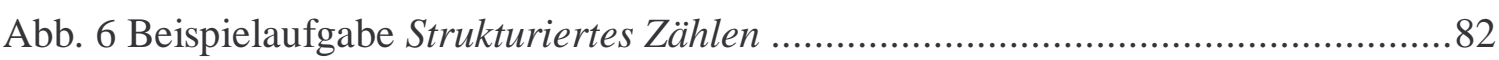

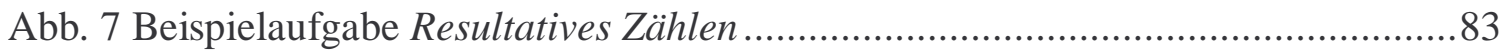

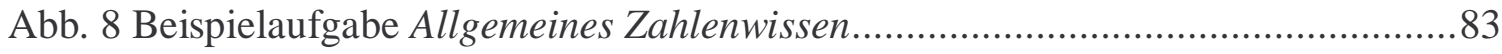

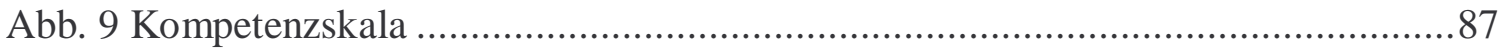

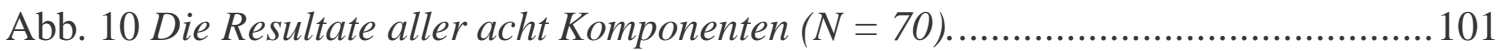

Abb. 11 Der Unterschied zwischen dem 1. und 2. Kindergartenjahr............................103

Abb. 12 Die Ergebnisse aller 40 Fragen in Prozent $(N=70)$.................................... 103

Abb. 13 Die Resultate der Komponente Vergleichsbegriffe ....................................... 105

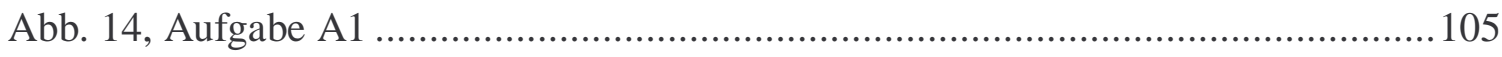

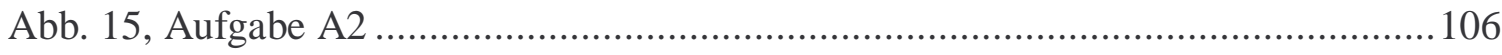

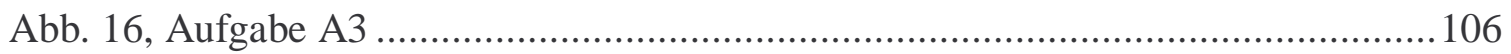

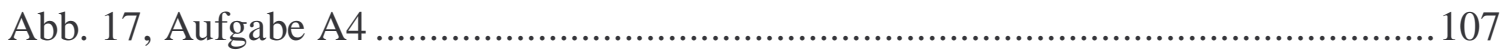

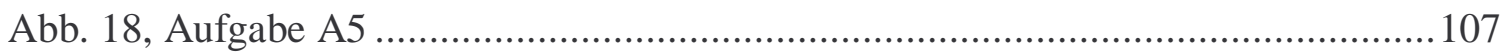

Abb. 19 Die großen Leistungsdifferenzen werden deutlich sichtbar............................ 108

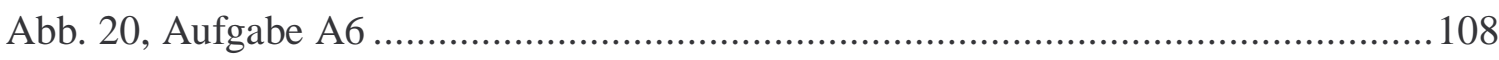

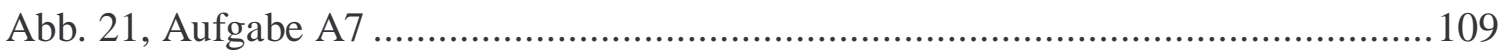

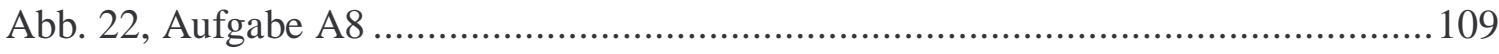

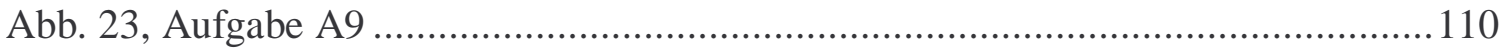

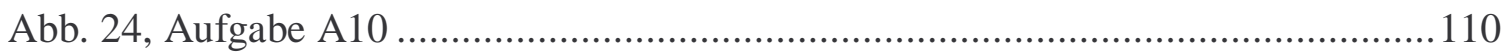

Abb. 25 Die Resultate fallen schön regelmäßig in der Komponente Zuordnungen. .......111

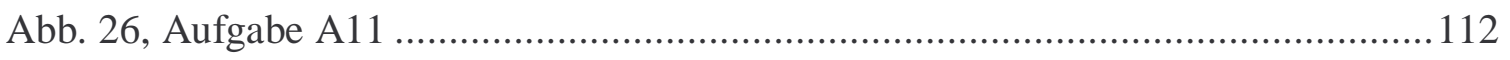

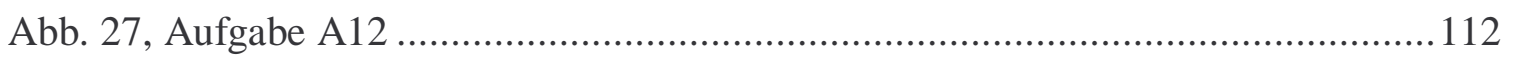

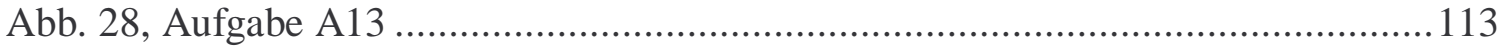

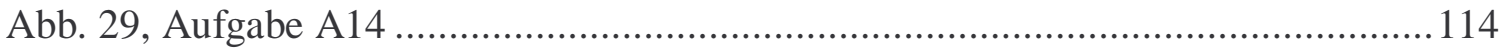

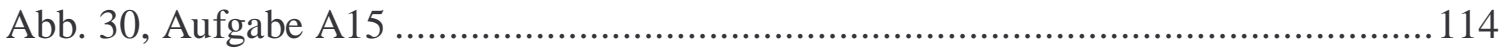

Abb. 31 Hier fallen die Ergebnisse nicht mit aufsteigender Aufgabennummer.............115 


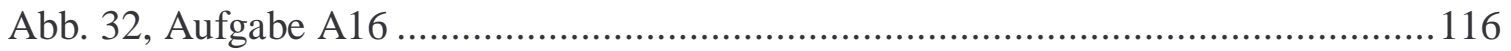

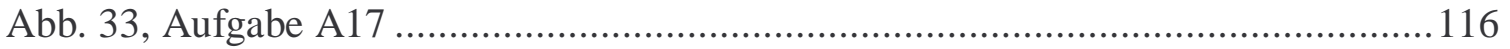

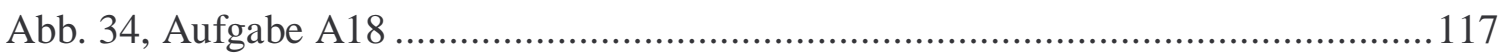

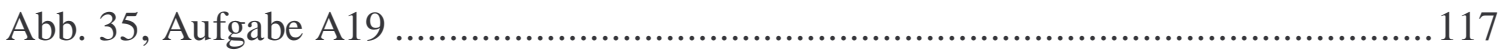

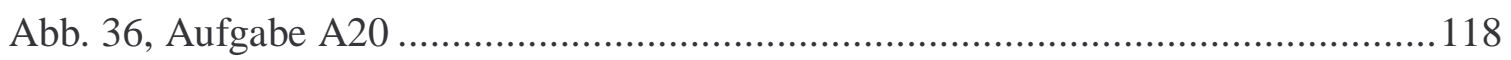

Abb. 37 Die Leistungsdifferenzen zwischen den Kindern..........................................119

Abb. 38 Das Leistungsgefälle kommt deutlich zum Ausdruck....................................120

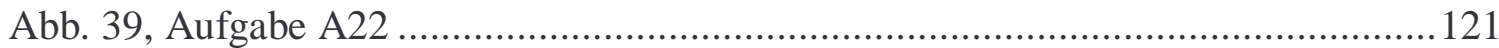

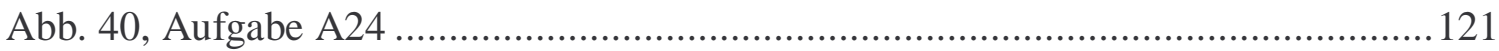

Abb. 41 Es handelt sich um Kindergartenkinder! ........................................................ 123

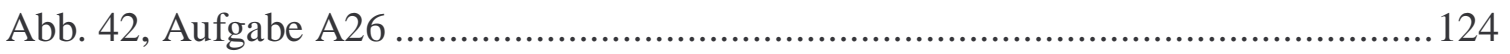

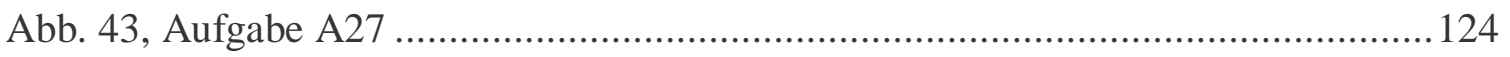

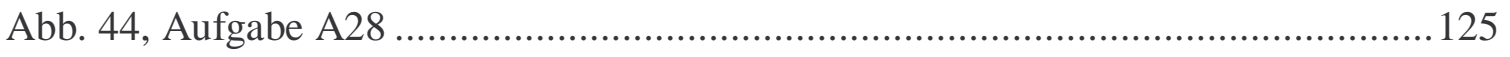

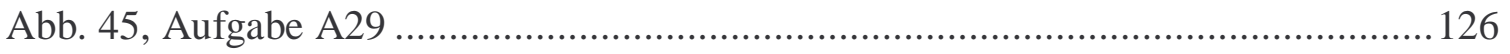

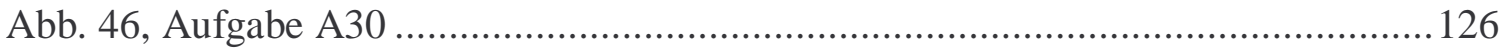

Abb. 47 Das ergebnisbezogene Zählen erfordert einen gefestigten Zahlbegriff............127

Abb. 48, Aufgabe A32 ….................................................................................... 128

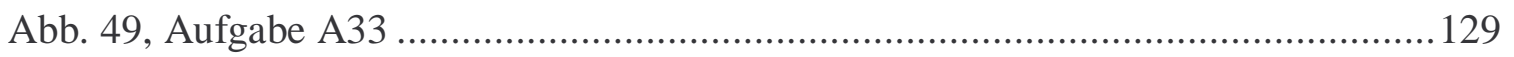

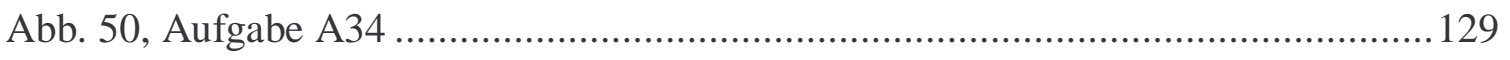

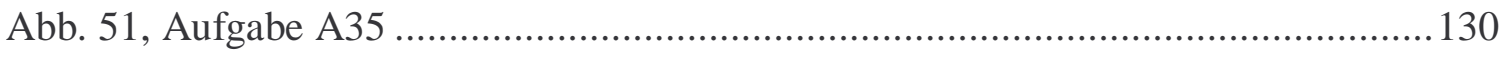

Abb. 52 Die letzte Komponente „Allgemeines Zahlenwissen“...................................131

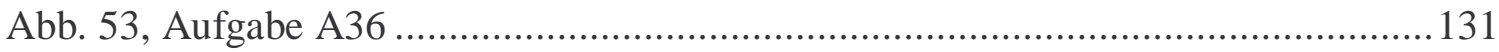

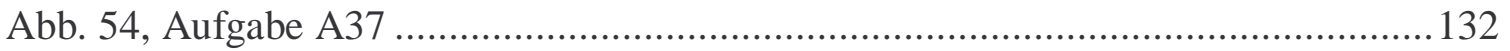

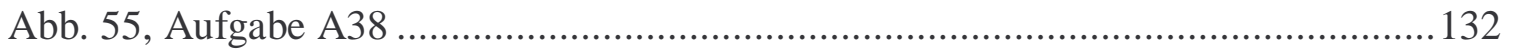

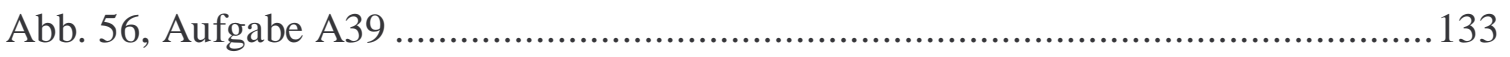

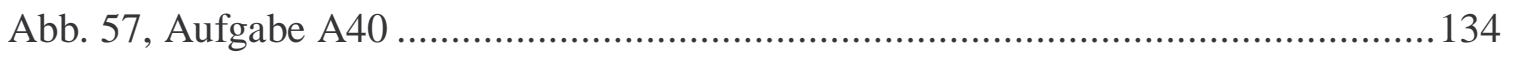

Abb. 58 Die Differenzen in den einzelnen Komponenten im Vergleich D - CH............139

Abb. 59 Eine schöne Verteilung nach rechts verschoben (siehe Hasemann 1998). ......140

Abb. 60 Auch hier eine schöne Verteilung............................................................ 141

Abb. 61 Anstieg der Zählkompetenz in Abhängigkeit zum Alter.................................196 


\section{Tabellenverzeichnis}

Tab. 1 Die Kinder werden in sechs Altersgruppen eingeteilt.

Tab. 2 Die Ergebnisse der holländischen Untersuchung nach Altersgruppen getrennt ..89

Tab. 3 Die Ergebnisse getrennt nach Kindergartenjahr. Maximalpunktzahl ist 100 .... 100

Tab. 4 Die Ergebnisse der 35 Mädchen und der 35 Buben liegen nahe beieinander. ... 100

Tab. 5 Die Ergebnisse der acht Komponenten $(N=70)$ 101

Tab. 6 Die Ergebnisse getrennt nach dem 1. und dem 2. Kindergartenjahr................... 102

Tab. 7 Die Ergebnisse getrennt nach dem 1. und dem 2. Kindergartenjahr.................. 104

Tab. 8 Alle Ergebnisse der Komponente Klassifizierung............................................ 108

Tab. 9 Die Resultate der Aufgaben 11 bis 15..........................................................111

Tab. 10 Ergebnisse: Einordnen in Reihenfolgen .................................................... 115

Tab. 11 Die Komponente „Gebrauch von Zahlwörtern“ ist für die Kleinen zu schwer. 119

Tab. 12 Die Resultate liegen bei Kindern im 2. KgJ beachtlich hoch. ..........................122

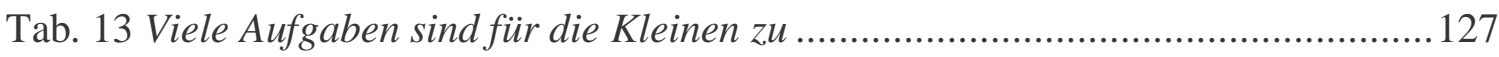

Tab. 14 Vergleichbare Mittelwerte der holländischen und Schweizer Kinder. ............. 137

Tab. 15 Die einzelnen Komponenten im Vergleich Deutschland - Schweiz.................. 138

Tab. 16 Die Punkteverteilung in Deutschland. Maximum sind 40 Punkte. $N=330 \ldots . . .140$

Tab. 17 Die Punkteverteilung in der Schweiz. Maximum sind 40 Punkte. $N=70 \ldots \ldots . . .140$

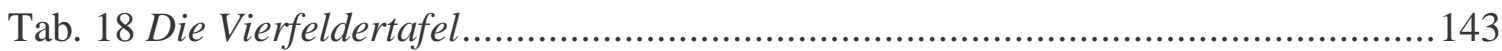

Tab. 19 Vierfeldertafel Klassifizierung - Zählkompetenz.........................................145

Tab. 20 Vierfeldertafel Zuordnungskomponente - Zählkompetenz ............................ 147

Tab. 21 Vierfeldertafel Zuordnungskomponente - Zählkompetenz (alternativ)............. 147

Tab. 22 Vierfeldertafel Reihenfolgen - Zählkompetenz ............................................ 148

Tab. 23 Vierfeldertafel Reihenfolgen - Zählkompetenz (alternativ) ............................149

Tab. 24 Trennschärfekoeffizienten der vier Komponenten und Zählkompetenz ............ 150

Tab. 25 Vierfeldertafel Alter $\rightarrow$ Vergleichskonzepte ................................................. 151

Tab. 26 Trennschärfekoeffizienten Alter und der vier Piaget'schen Komponenten ........151

Tab. 27 Vierfeldertafel Leistungsfähigkeit $\rightarrow$ Vergleichskonzepte ............................ 152

Tab. 28 Trennschärfekoeffizienten Leistungsfähigkeit und der vier Komponenten .......152

Tab. 29 Vierfeldertafel Alter $\rightarrow$ Zählkompetenz.................................................. 153

Tab. 30 Vierfeldertafel Leistungsfähigkeit $\rightarrow$ Zählkompetenz .................................153

Tab. 31 Zusammenstellung Korrelationskoeffizienten ............................................... 154 


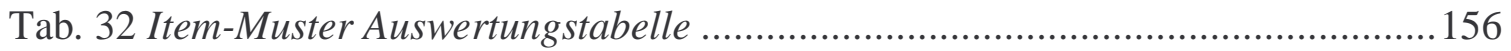

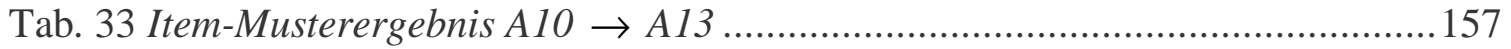

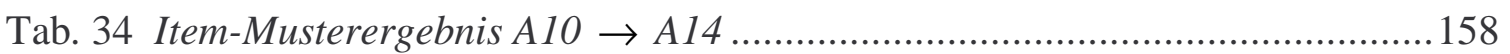

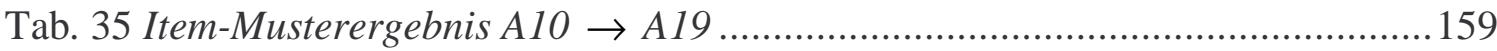

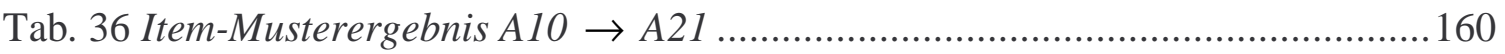

Tab. 37 Durchschnittsalter der drei Gruppen ....................................................... 161

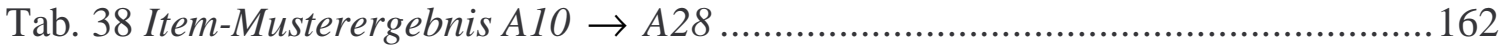

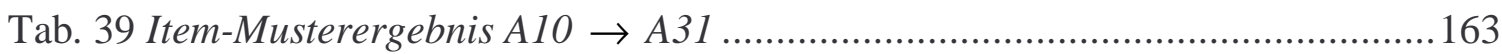

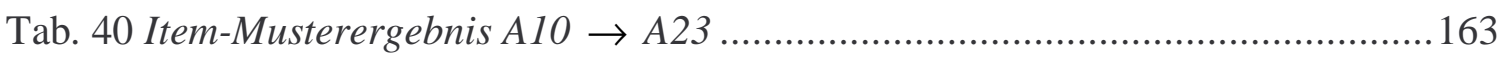

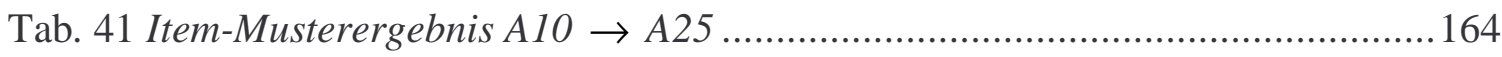

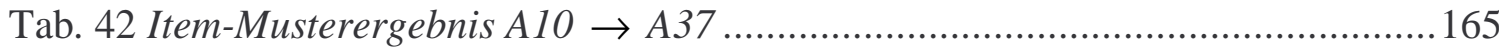

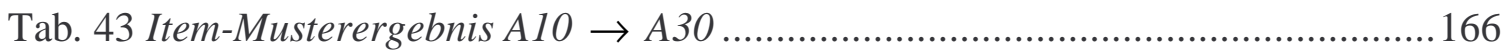

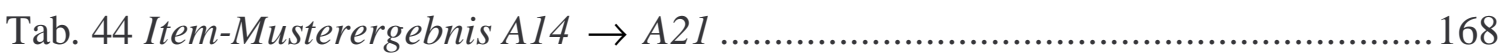

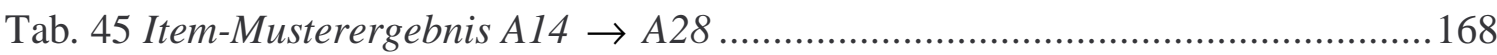

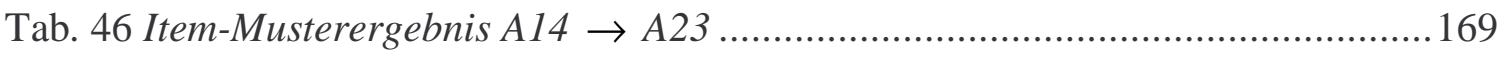

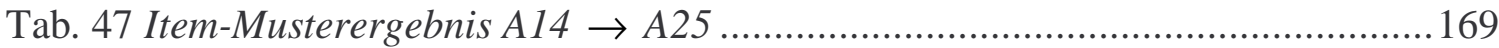

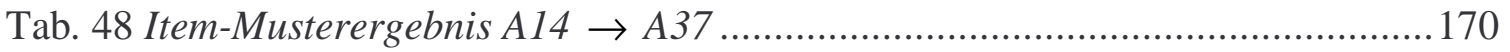

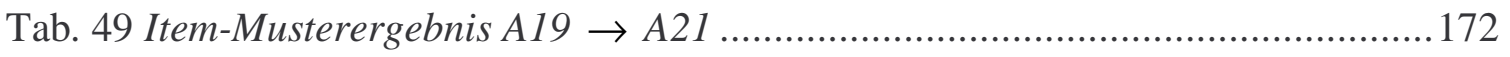

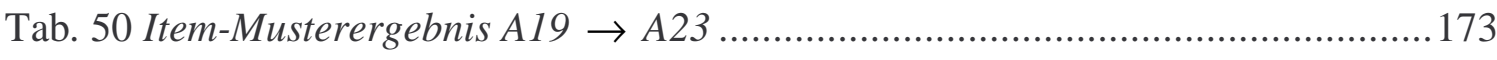

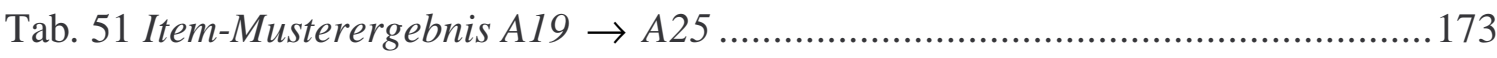

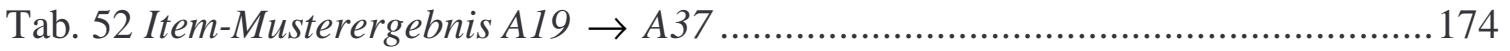

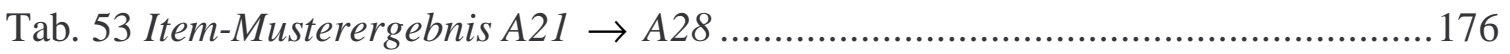

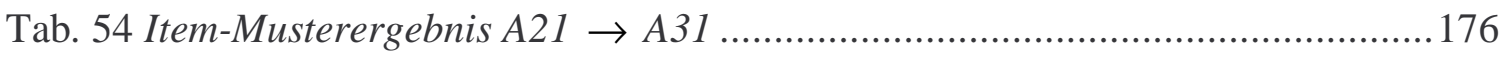

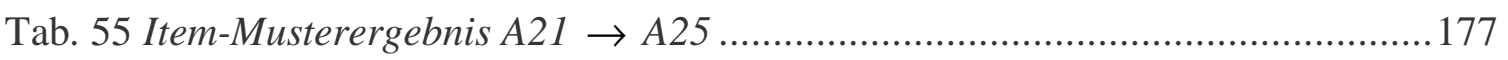

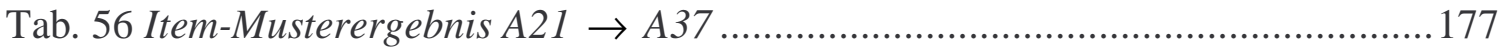

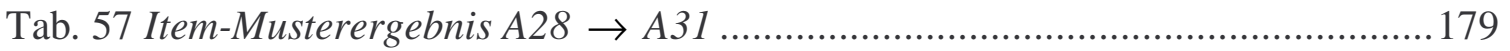

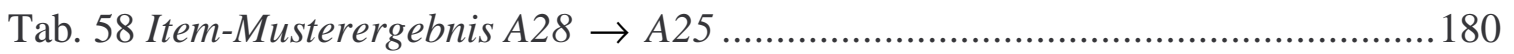

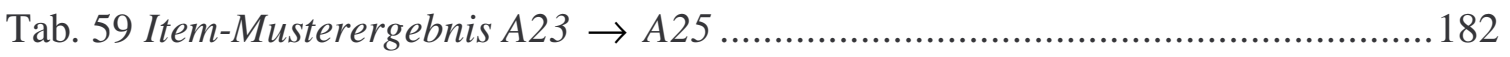

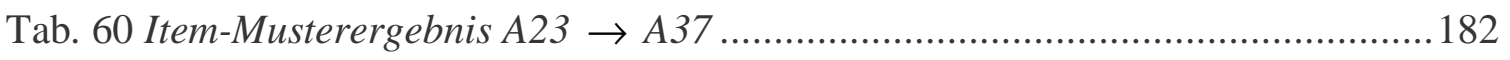

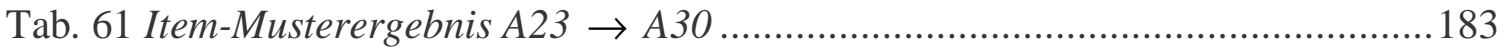

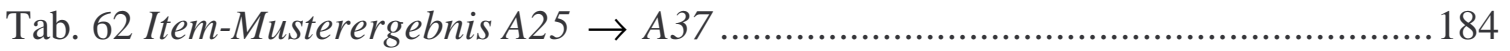

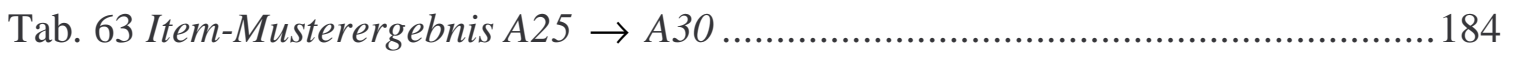

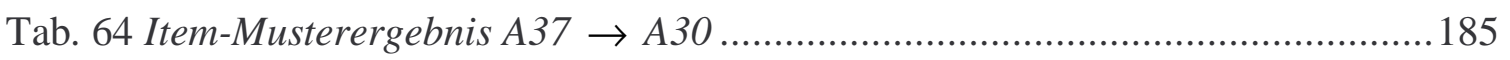


Tabellenverzeichnis

Tab. 65 Enorme Leistungsdifferenzen bei den ganz jungen Kindern ..........................210

Tab. 66 Das gleiche Phänomen bei den älteren Kindern ..........................................2211 


\section{Literaturverzeichnis}

Aargauer Lehrmittel Mathematik 1. Schuljahr, Lehrmittelverlag des Kantons Aargau 1995.

Antell, E., Keating, D.P.: Perception of numerical invariance in neonates. Child development, 54, 1983, 695-701,.

Aristoteles: Metaphysik. Übersetzt und hrsg. von Franz F. Schwarz. Stuttgart 1970.

Baroody, A.J.: The relationship among the development of counting, number conservation, and basic abilities, in: Dissertation Abstracts International, A 39, $1979,6640-6641$.

Baroody, A.J.:Procédures et principe de comptage: leur développement avant l'école. In: Bideaud, J. et al. (Hrsg.): Les chemins du nombre. Lille 1991, 133158.

Baroody, A.J.: The relationship between the order-irrelevance principle and counting skills. In: Journal for Research in Mathematics Education 24 (1993) 5, 415-427.

Bauersfeld, H.: Subjektive Erfahrungsbereiche als Grundlage einer Interaktionstheorie des Mathematiklehrens und -lernens. In: Heinrich Bauersfeld u.a.: Lehren und Lernen von Mathematik. Köln: Aulis IDM-Reihe, Bd 6, (1983) 5, $1-56$.

Beth, E.W.; Piaget, J.: Mathematical epistemology and psychology. Dordrecht 1966,

Botkin, J.W.: No Limits to Learning, Oxford 1979.

Brainerd, Ch. J.: The origins of number concept. New York/London/Sydney/Toronto 1979.

Briars, D.; Siegler, R.S.: A featural analysis of preschoolers' counting knowledge. Developmental Psychology 20 (1984) 607-618.

Brissiaud, R.: Toward a Vygotskian approch to the psychogenesis of number. In: Cahier de Psychologie Cognitive 14 (1995) 6, 695-702.

Brown, J; Collins, A.; Duguid, R.: Situated Cognition and the Culture of Learning, Educational Researcher 1989.

Bryant, P.: $\quad$ Mathematical understanding in the nursery school years. In: Nunes, T.; Bryant, P.: Learning and teaching mathematics. Sussex 1997, 53-67.

Bundschuh, K.: Pädagogische Psychologie. München/Basel 1995. 
Carey, S.: $\quad$ Semantic development: The state of art. In E. Wanner \& L.R. Gleitman (Eds.), Language acquisition: The state of art (pp. 347-389). Cambridge: Cambridge University Press 1982.

Carraher, T.N.; Carraher, D.W. \& Schliemann, A.D.: Mathematics in the streets and schools. British Journal of Developmental Psychology 1985 3, 21-29.

Comiti, $C .: \quad$ Les premiers acquisitions de la notation de nombre par l'enfant, in: Educational Studies in Mathematics Education (1980) 11, 301-318.

Cowan, R.: $\quad$ Performanc in number conservation tasks as a function of the number of items. British Journal of Psychology 70 (1979), 77-81.

Chomsky, N.: Aspekte der Syntay-Theorie. Frankfurt Suhrkamp 1973.

Church, R.M.; Meck, W.H.: The numerical attribute of stimuli. In: H.L. Roitblat, T.G. Bever \& H.S. Terrace (Hg.) Animal Cognition. Hillsdale, NJ: Erlbaum 1984.

Davidson, J.E.; Sternberg, R.J.: Competence and Performance in Intellectual Development. In: Neimark, E.D.: Moderators of Competence. New York 1985, 43-76.

Dehaene, S.: Der Zahlensinn oder warum wir rechnen können. Basel Birkhäuser 1999.

Donaldson, M.: Wie Kinder denken. Bern 1982.

Dutge, S.: $\quad$ Mentale Modelle: Konstrukte des Wissens und Verstehens - Kognitionspsychologische Grundlagen für die Software- Ergonomie, Verlag für Angewandte Psychologie, Göttingen 1994.

Edelmann, W.: Lernpsychologie. 5., vollst. überarbeitete Auflage; Beltz PsychologieVerlags-Union, Weinheim, Basel 1996.

EDK-Dossier 48A: Bildung und Erziehung der vier- bis achtjährigen Kinder in der Schweiz 1997.

EDK-Dossier 57A: Die Ausbildung von Lehrpersonen für die Basisstufe 1999.

Elschenbroich, D.: Weltwissen der Siebenjährigen, Kunstmann, München 2002.

Fischer, J.-P.:L'enfant et le comptage, I.R.E.M. Straßburg 1982.

Flammer, A.: Entwicklungstheorien. Psychologische Theorien der menschlichen Entwicklung. Bern/Toronto/Göttingen 1996.

Franke, M.; Edler, S., Kettner, B.; Kilian, A. \& Ruwisch, S.: Kinder bearbeiten Sachsituationen in Bild-Text-Darstellung. Journal für Mathematikdidaktik (1998) 19, 89-122. 
Fragnière N. e.a.: Arithmetische Fähigkeiten im Kindergartenalter. Klett und Balmer, Zug 1999.

Freudenthal, H.: Mathematik als pädagogische Aufgabe, Bd. 1 Stuttgart 1973.

Frydman, $O$; Bryant, P.: Sharing and the understanding of number equivalence by young children. In: Cognitive Development, (1988) 3, 323-339.

Frydman, O.: The concept of number and the acquisition of counting concepts: The , when', the ,how' and the 'what' of it. In: Cahiers de Psychologie Cognitive 14 (1995a) 653-684.

Frydman, O.: Knowledge about number and knowledge about knowledge: The insides and outsides of our windows. In: Cahiers de Psychologie Cognitive 14 (1995b) 764-774.

Frye, D.: Cognitive development, intention and instruction. In: Cognitive Development 12, 1997, 315-321.

Fuchshuber, A.: zwei und mehr. St. Gabriel Verlag, Mödling - Wien 1997.

Fuson, K.C.: An analysis of the counting-on solution procedure in addition, In: T.P. Carpenter-J.M. Moser-T.A. Romberg (Eds.): Addition and Subtraction: A Cognitive Perspective, Hillsdale, N.J. 1982, 67-81.

Fuson, K.C.: Children's counting and concepts of number. New York 1988.

Fuson, K.C.: Aspects and uses of counting: An AUC framework for considering research on counting to update the Gelman/Gallistel counting principles. In: Cahiers de Psychologie Cognitive (1995) 14, 724-731.

Geary, D.C.: Children's mathematical development. Research and practical applications. Washington, DC: American Psychological Association 1994.

Geary, D.C.: Refelction of evolution and culture in children's cognition. American Psycholgist, (1995) 50(1), 24-37.

Gallistel, C.R.: The organisation of learning. Cambridge, MA: Bradford Books/MIT Press 1990.

Gallistel, C.R.; Gelman, R.: Preverbal and verbal counting and computation. In: Dehaene, S. (Hrsg.): Numerical cognition. Oxford/Cambridge 1993, 43 74.

Geary, D.C.: Reflections of evolution and culture in Children's cognition. American Psycholgist (1995) 50(1), 24-37. 
Gelman, R.: First principles organize attention to and learning about relevant data: Number and the animate-inanimate distinction as examples. Cognitive Science. (1990) 14, 79-106.

Gelman, R.: Constructing and using conceptual competence. In: Cognitive Development 12 1997, 305-313.

Gelman, R.; Baillargeon, R.: A review of somme Piagetian concepts. In P.H. Mussen (Ed.), Handbook of child psychology (Vol. III, ppp 167-230). New York: Wiley 1983.

Gelman, R.; Gallistel, C.R.: The child's understanding of number. Cambridge, MA: Harvard University Press 1978.

Gelman, R.; Greeno, J.G.: On the nature of competence: Principles for understanding in a domain. In L.B. Resnick (Ed.), Knowing, learning and instruction: Essays in honour of Robert Glaser (pp. 125-186). Hillsdale, NJ: Erlbaum 1989.

Gelman, R.; Meck, E.: The notion of principle: The case of counting. In J. Hiebert (Ed.), Conceptual and procedural knowledge: The case of mathematics (pp. 2957). Hillsdale, NJ: Erlbaum 1986.

Gelman, R.; Meck, E.: Early principles aid initial but not later conceptions of number. In: Bideaud, J. et al (Hrsg.): Pathway to number. Hillsdale/New Jersey/London 1992, 171-189.

GEO-Wissen: Denken Lernen Schule Gruner + Jahr 1/1999, 182.

Gerster, H.-D.: Arithmetik im Anfangsunterricht. In A. Abele/H. Kalmbach (Hg.), Handbuch der Grundschulmathematik. 1. und 2. Schuljahr, S. 35-102. Bd. 1. Stuttgart 1994.

Ginsburg, H; Opper, S.: Piagets Theorie der geistigen Entwicklung. Stuttgart 1998.

Ginsburg, H.: Children's arithmetic: The learning process. New York: Van Nostrand 1977.

Ginsburg, H.: Protocol methods in research on mathematical thinking. In: Ginsburg, H. (Hrsg): The development of mathematical thinking. New York: Academic Press 1983, 7-47.

Glatfeld, M.: Der Zahlbegriff in Primar- und Orientierungsstufe, in: Sachunterricht und Mathematik in der Grundschule (1976) 4, 141-148.

Grassmann, M. u.a.: Arithmetische Kompetenz von Schulanfängern. In: Sachunterricht und Mathematik in der Primarstufe (1995) 23, 314-321. 
Greeno, J.G.: Situations, mental models and generative knowledge. In D. Klahr \& K. Kotovsky (Eds.), Complex information processing: The impact of Herbert A. Simon. Hillsdale, NJ: Erdbaum 1989, 285-318.

Greeno, J.G., Smith, D.R., Moore, J.L.: Transfer of situated learning. In D.K. Detterman \& R.J. Sternberg (Eds.), Transfer on trial: Intelligence, cognition and instruction. Norwood, NJ: Ablex Publishing Corp. 1993, 99-167.

Grob, B.: Verweigern sich Kindergärtnerinnen kognitiven Leistungen, wenn ja, weshalb? Unveröffentlichte Lizentiatsarbeit Universität Bern 2001.

Grotjahn, R.: Testtheorie: Grundzüge und Anwendungen in der Praxis. In: Wolff, A.; Tanzer, H. (eds.): Sprache-Kultur-Politik. Regensburg 2000.

Grüssing, M.: Entwicklung mathematischer Kompetenzen im Grundschulalter. Beiträge zum Mathematikunterricht. DIV-Verlag Franzbecker 2002, 199-202.

Hagendorf, H.: Cognitive Performance and Developmental Constraints, In Schneider/Weinert, 1990.

Hasemann, K.: Mathematische Lernprozesse. Analysen mit kognitionstheoretischen Modellen. Vieweg. Braunschweig/Wiesbaden 1986.

Hasemann, K.: Die frühe mathematische Kompetenz von Kindergartenkindern und Schulanfängern - Ergebnisse einer empirischen Untersuchung. In Beiträge zum Mathematikunterricht, Franzbecker Verlag 1998, 263-266.

Hasemann, K.: Buchmanuskript, Spektrum-Verlag, Heidelberg. Erscheint voraussichtlich 2003.

Hasemann, K.: Mathematisches Wissen und Verstehen im Vor- und Grundschulalter. Diagnose, Hemmnisse und Entwicklung. Symposium Bamberg, Vortragsmanuskript 2003a.

Hasebrook, J.: Multimedia-Psychologie: Eine neue Perspektive menschlicher Kommunikation. Spektrum Verlag, Heidelberg, Berlin, Oxford 1995.

Helferich, Ch.: Geschichte der Philosophie. dtv München 1998.

Hengartner E./Röthlisberger H.: Rechtsfähigkeit von Schulanfänger. In: Schweizer Schule 4/1994, S. 3-25.

Hengartner E./Röthlisberger H.: Standorte und Denkwege von Kindern: Erkundungsprojekte in der fachdidaktischen Ausbildung. UVK, St. Gallen 1995.

Hengartner E.: Mit Kindern lernen S. 133.

Lehrplan Kindergarten Kanton Basel-Landschaft Lehrmittelverlag Liestal 1998.

Ifrah, G.: $\quad$ Universalgeschichte der Zahlen. Frankfurt/M 1989. 
Ifrah, G.: $\quad$ Die Zahlen. Frankfurt/M 1992.

Ingenkamp, K.: Lehrbuch der Pädagogischen Diagnostik (Studienausgabe. Weinheim \& Basel: Belz 1985.

Jandl,E.; Junge, N.: fünfter sein. Beltz \& Gelberg, Weinheim und Basel 2001.

Janosch: $\quad$ Das große Buch der Kinderreime. Diogenes, Zürich 1984.

Käpnick, F.: Mathematisch begabte Kinder: Modelle, Empirische Studien und Forderungsprojekte für das Grundschulalter. Greifswalder Studien zur Erziehungswissenschaft 1998.

Käpnick, F.: „Aber Zahlen dürften nicht immer nur ein System haben“- Zur Entwicklung subjektiver Zahlauffassungen bei Grundschülern. In Beiträge zum Mathematikunterricht. Tagungsbericht div-Verlag Franzbeck 1999, 273276.

Käpnick, F.: „But Figures aren’t supposed to have one System only - About the Development of Subjective Number Conceptions of Elementary-School Children. Selected Papers from the Annual Conference on Didactics of Mathematics, div-Verlag Franzbecker 1999, 33-43.

Kesselring T: Jean Piaget, München 1999.

Klahr, D.: Transition processes in quantitative development. In R. Sternberg (Ed.), Mechanisms of cognitive development, 112-125. San Francisco: Freeman, 1984.

Klahr, D. \& Wallace, J.G.: Cognitive development: An information processing view. Hillsdale, NJ: Erlbaum, 1976.

Klein, A.; Starkey, P.: The origins and development of numerical cognition: A comparative analysis. In J.A. Sloboda \& D. Rogers (Eds.), Cognitive processes in mathematics (pp. 1-25). Oxford: Clarendon Press. 1987.

Kline, M.: $\quad$ Warum kann Hänschen nicht rechnen? Das Versagen der neuen Mathematik. Weinheim/Basel 1974.

Kline, M.: $\quad$ Mathematical thought from ancient to modern times. New York/Oxford 1990.

Koch, R.: $\quad$ Mathematische Ausbildung angehender Kindergärtnerinnen und Kindergärtner am Lehrerseminar Liestal. In: Basellandschaftliche Schulnachrichten, 2, 2000.

Kofsky, E.: Developmental scalogram ananysis of classificatory behavior, Phil. Diss., Rochester N.Y. 1963. 
Kofsky, E.: A scalogram study of classificatory development, in: Child Development 1966, 37, 191 -. 204,

Krauthausen, G.: Lernen-Lehren-Lehren lernen. Leipzig Stuttgart Düsseldorf 1998.

Krauthausen, G.; Scherer, P.: Einführung in die Mathematikdidaktik, Spektrum Verlag Heidelberg Berlin 2001.

Kuhn, T.S.: $\quad$ Die Struktur wissenschaftlicher Revolutionen. Bd. 1 Leipzig 1989.

Kunz, G.; Drewniak, U.: Zur differentiellen Bedeutung kognitiver, metakognitiver und motivationaler Variablen für das effektive Lernen mit Instruktionetexten und Bildern. In: Mandl, H. (Hrsg.) 1992.

Lave, J.; Wenger, E.: Situated Learning: Legitimate Peripheral Participation, Cambridge University Press 1991.

Lehrplan Kindergarten Kanton Basel-Landschaft Lehrmittelverlag Liestal 1999.

Lienert, G.; Raatz, U.: Testaufbau und Testanalyse. Weinheim: Belz, Psychologie Verlags Union 1998.

Lorenz, J.H.; Radatz, H.: Handbuch des Förderns im Mathematikunterricht. Hannover, 1994.

Maier, H.: $\quad$ Didaktik des Zahlbegriffs, Hannover 1990.

Mandl, H. (Hrsg.): Lern- und Denkstrategien. Göttingen: Hogrefe 1992.

Mani, K.; Johnson-Laird, P.N.: The mental representation of spatial descriptions, "Memory and Cognition" 1982, 10, S. 181-187,.

Meck, W.H.; Church, R.M.: A mode control model of counting and timing processes. In: Journal of Experimental Psychology. Animal Behaviour Processes 9, 1983, 320-334.

Mehler, J., \& Bever, T.G.: Cognitive capacity of very young children. Science 1997, $158,141-142$.

Moser Opitz, E.: Zählen, Zahlbegriff, Rechnen. Theoretische Grundlagen und eine empirische Untersuchung zum mathematischen Erstunterricht in Sonderklassen. Bern, Stuttgart, Wien. Haupt 2001.

Mpiangu, B.D.; Gentile, R.J.: Is conservation of number a necessary condition for mathematical understanding?, in: Journal for Research in Mathematics Education 1975, 6, 179-192.

H. van Luit, B. van de Rijt, K. Hasemann: Osnabrücker Test zur Zahlbegriffsentwicklung Hogrefe, Göttingen 2001. 
Niegemann, H.M.: Computergestützte Instruktion in Schule, Aus- und Weiterbildung: Theoretische Grundlagen, empirische Befunde und Probleme der Entwicklung von Lernprogrammen. P. Lang, Frankfurt 1995.

OTZ: $\quad$ Osnabrücker Test zur Zahlbegriffsentwicklung, Van Luit J.E.M., Van de Rijt B.A.M., Hasemann K., Hogrefe, Göttingen 2001.

Papert, S.: $\quad$ Problèmes épistémologiques et génétiques de la récurrence. In: Greco, P., Grize, J.-B. Papert, S., \& Piaget, J., Études d’épistémologie Génétique. Band 11. Problèmes de la construction du nombre. S. 117-148. Paris : Presses Universitaires de France 1960.

Parrat-Dayan, S.: Task analysis and the organisation of knowledge in children. In: Cahiers de Psychologie Cognitive 14 (1995) 6, 750-752.

Pennington, B.F.: What Piaget's conservation of number tasks doesn't tell us about a child's understanding of numerical invariance and arithmetic, in: Dissertation Abstracts International 1977, B38, 1897-1898.

Piaget, J.: The child's conception of number. New York, Norton 1965.

Piaget, J.: $\quad$ Genese und Struktur in der Psychologie der Intelligenz. In: Theorien und Methoden [2b] 1966, 265 - 277,

Piaget, J.: $\quad$ Der Strukturalismus. Olten/Freiburg 1973.

Piaget, J.: $\quad$ Einführung in die genetische Erkenntnistheorie, Frankfurt 1973a.

Piaget, J.: $\quad$ Die Zukunft unseres Bildungssystems. In: J. Piaget: Das Recht auf Erziehung und Die Zukunft unseres Bildungssystems. Zwei Essays. München: Piper (Serie Piper 128) 1975.

Piaget, J.: $\quad$ Das Erwachen der Intelligenz beim Kinde, Stuttgart 1975a.

Piaget, J.; Inhelder, B.: Die Entwicklung der elementaren logischen Strukturen (Teil I und II), Düsseldorf 1973.

Piaget, J.; Inhelder, B.: Die Entwicklung des räumlichen Denkens beim Kinde. Stuttgart, Klett 1975.

Piaget J./Szeminska A.: Die Entwicklung des Zahlbegriffs beim Kinde Stuttgart 1975b (Übersetzung der französischen Originalausgabe: La genèse du nombre chez l’enfant, Neuchâtel 1967).

Piaget, J.; Inhelder B.: Die Entwicklung des inneren Bildes beim Kind. Frankfurt 1979.

Piaget, J.; Inhelder B.: Die Psychologie des Kindes. Frankfurt/M 1981.

Piaget, J.: $\quad$ Biologie und Erkenntnis. Frankfurt/M 1992.

Piaget, J.: Über Pädagogik. Weinheim/Basel 1999. 
Preiß, G.: $\quad$ Projekt für den Kindergarten: Entdeckungen im Zahlenland. Unveröffentlicht.

Pressley, M.; Wood, W.; Woloshyn, V.: Elaborative interrogation constraints. In: Schneider/Weinert 1990.

Preussler, O.; Lenz, L.: Die dumme Augustine, Thienemanns Verlag Stuttgart.

Rabinowitz, M.; Goldberg, N.: Evaluating the structure-process hypothesis. In: Schneider/Weinert 1995.

Radat, $H .: \quad$ Hilfreiche und weniger hilfreiche Arbeitsmittel im mathematischen Anfangsunterricht. Grundschule (1991) 24, 46-49.

Radatz, H.: Ikonomanie. Oder: Wie sinnvoll sind die vielen Veranschaulichungen im Mathematikunterricht? Grundschulmagazin (1993) 3, 50-51.

Radatz, H.; Schipper, W.: Handbuch für den Mathematikunterricht an Grundschulen. Hannover 1983.

Radatz, H. et al.: Handbuch für den Mathematikunterricht - 2. Schuljahr. Hannover 1998.

Reinmann-Rothmeier, G. \& Mandl, H.: Lehren im Erwachsenenalter. Auffassungen vom Lehren und Lernen, Prinzipien und Methoden. In: Weinert F.E. und Mandl H. (Hrsg.), Psychologie der Erwachsenenbildung (Enzyklopädie der Psychologie, Themenbereich D, Serie I, Band 4) S. 355-404. Göttingen: Hogrefe 1997.

Resnick, L.B.: Developing mathematical knowledge. In American Psychologist 44 (1989) 2, 162-169.

Rickmeyer, K.: Die Zwölf liegt hinter der nächsten Kurve und die Sieben ist pinkrot: Zahlenraumbilder und bunte Zahlen. In: Journal für Mathematik-Didaktik JMD 2001, Heft 1, 51-71,.

Rogoff, B.: $\quad$ Apprenticeship in thinking. New York: Oxford University Press 1990.

Rumelhart, D.E; Ortony, A.: Schemata: The building blocks of cognition. In R.J. Spiro, B.B. Bruce and W.F. Brewer (Ed.), Theoretical issues in reading comprehension. Hillsdale, NJ: Erdbaum 1977, 33-58.

Saxe, G.B., \& Gearhart, M.: Children's mathematics. San Francisco: Jossey-Bass 1988. Saxe, G.B.: $\quad$ Development relations between notational counting and number conservation. In: Child Development 1979, 50, 180-187. 
Saxe, G.B.: $\quad$ Emergent mathematical environment in children's games. In: Steffe, L.P. et al (Hrsg.): Theories of mathematical learning. Mahwah/New Jersey 1996, 51-68.

Schank, R.C, Abelson, R.P.: Plans, goals and understanding: An inquiry into human knowledge structures. Hilldsdale, NJ: Erdbaum 1977.

Schipper, W.: Stoffauswahl und Stoffanordnung im mathematischen Anfangsunterricht. Journal Mathematikdidaktik 1982, S. 91-120.

Schlechtweg, W.; Buchmann, G.: Endliche Mengen, 3. Aufl., Freiburg/Br. 1970.

Schmassmann M.: Mathematik im Kindergarten: Wirklichkeit abstrahierend ordnen. In: Schweizer Kindergarten 1986(76) 1, 10-17.

Schmidt, S.; Weiser, W.: Zählen und Zahlverständnis von Schulanfängern. Journal Mathematikdidaktik 1982, 3/4, 227-236.

Schmidt, S.: Was soll den Grundschullehrerinnen und Lehrern an fachdidaktischem Wissen zum arithmetischen Anfangsunterricht vermittelt werden? In: ZDM 1992/2, S. 50-60.

Schneider, W.; Weinert, F.E.: (Hrsg.) Interactions among aptitudes, strategies, and knowledge in cognitive performance. New York, Springer 1990.

Schneider, W.; Weinert, F.E.: (Hrsg.) Memory performance and competencies. Mahwah: Erlbaum 1995.

Schroedel-Verlag: Arithmetische Fähigkeiten am Schulanfang. Test im Rahmen der Erprobung von DIE WELT DER ZAHL am Schuljahresbegin 96/97. http://www.schroedel.de/noframe/lehrer/gs/zusatz/test/test1.html, 27.3.1998.

Schröder, E.: Vom konkreten zum formalen Denken. Bern/Stuttgart 1989.

Schulz, R.: $\quad$ Kompetenz versus Performanzkognitive Lernprozesse und ihre Konsequenzen für die Fremdsprachendidaktik, Hausarbeit Universität Potsdam 1998.

Schwirtz, W.: Zur Bedeutung des Phänomens der Mengeninvarianz für den mathematischen Anfangsunterricht, in: Beiträge zur Mathematikdidaktik. Festschrift für Wilhelm Oehl, 101-110, Hannover 1976.

Selter C./Spiegel H.: Wie Kinder rechnen. Stuttgart 1997.

Selter $C$.: $\quad$ Die Fiktivität der „Stunde Null“ im arithmetischen Anfangsunterricht. In Mathematische Unterrichtspraxis 1995, 2, 11-19. 
Siegler, R.S.: Developmental sequences within and between concepts. Monographs of the Society for Research in Child Development 1981, 46(2, Serial No. 189).

Siegler, R.S.: Beyond competence - Toward development. In: Cognitive Development 1997, 12, 323-332.

Skemp, R.R.: Mathematics in the primary school. London: Routledge 1989.

Spiegel, H.: Zahlenkenntnisse von Kindern bei Schuleintritt In: Sachunterricht und Mathematik in der Primarstufe (1979) H. 6 S. 227 - 244 und H.7 S. 275 278.

Spiegel, H.: Was und wie Kinder zu Schulbeginn schon rechnen können. Ein Bericht über Interviews mit Schulanfängern. In: Grundschulunterricht (1992) H.11, S. 21-23.

Spiro, R.; Feltovich, R.; Jacobson, M.; Coulson, R.: Cognitive Flexibility, Constructivism and Hypertext: Random Access Instruction for Advanced Knowledge Acquisition in Ill_Structured Domains. Educational Technology 1991.

Soja, NN., Carey, S., \& Spelke, E.S.: Ontological categories guide young children's indcutions of word meaning: Object terms and substance terms. Cognition 1991, 38, 179-211.

Sophien, C.: Limitations on preschool children's knowledge about counting: Use counting to compare two sets. In: Development Psychology 1988a 24 5, 634-640.

Sophian, C.: Early developments in children's understanding of number: Inferences about numerosity and one-to-one correspondence. In Child Development 1988b 59, 1397-1414.

Sophian, C.: Learning about numbers: Lessons for mathematics education from preschool number development. In: Bideaud, J. (Hrsg.): Pathway to number. Children's developing numerical abilities. Hillsdale/New Jersey/London 1992, 19-40.

Sophian, C.: The trouble with the competence models. In: Cahiers de Psychologie Cognitive 1995, 14, 753-759.

Sophian, C.: Beyond competence: The significance of performance for conceptual development. In: Cognitive Development 12 1997a, 281-303,. 
Sophian, C.: Growing points for cognitive-developmental theories: Characterising innate foundations for learning. In: Cognitive Development 12 1997b, 345$348,$.

Stamm, M.: $\quad$ Basellandschaftliche Schulnachrichten, Nr.1 Febr. 1999.

Starkey, P., Spelke, E.S., Gelman, R.: Numerical abstraction by human infants. Cognition $1990,36,97-127$.

Stendler-Lavatelli, C.: Früherziehung nach Piaget. München/Basel 1976.

Stern, E.: $\quad$ Wieviel Kinder bekommen keinen Mohrenkopf? In: Zeitschrift für Entwicklungspsychologie und pädagogische Psychologie 1994, 24, S. 79 94.

Stern, E.: $\quad$ Die Entwicklung des mathematischen Verständnisses im Kindesalter.

Lengrich/Berlin/Düsseldorf/Leipzig 1998.

Strittmatter, A.: „Ja, aber“ zur Basisstufe in LCH-Aktuell 1998.

Tages-Anzeiger vom 11. 8. 1999, Zürich.

Van de Rijt, B.A.M., Van Luit, J.E.H. \& Pennings, A.H.: The construction of the Utrecht early mathematical competence scales. Educational and Psychological Measurement 1994, 59(2), 289-309.

Van de Rijt, B.A.M.: Voorbereidende rekenvaardgheid bij kleuters. Doetinchem: Graviant. 1996.

Van Luit J.E.M., Van de Rijt B.A.M., Hasemann K.: Zur Messung der frühen mathematischen Kompetenz. In Zeitschrift für Entwicklungspsychologie und Pädagogische Psychologie, 2000, 32(1), 14-24.

Van Luit J.E.M., Van de Rijt B.A.M., Hasemann K.: Osnabrücker Test zur Zahlbegriffsentwicklung, Hogrefe, Göttingen 2001.

Van den Heuvel-Panhuizen M.: Leistungsmessung im aktiv-entdeckenden Mathematikunterricht. In Brügelmann, H.u.a. (Hg.): Am Rande der Schrift. Jahrbuch der Dt. Gesellschaft Lesen und Schreiben 6. Bottighofen: Lybelle Verlag 1995, S. $66-86$.

Van der Meer, E.: Dynamics of Knowledge Structures: Some Underlying Strategies. In: Schneider/Weinert 1990.

Voigt, J.: Die mikroethnographische Erkundung von Mathematikunterricht - Interpretative Methoden der Interaktionsanalyse. In: Maier, H./Voigt, J. Interpretative Unterrichtsforschung. IDM 17, Aulis Verlag, Köln 1991, S. 152-175. 
Wachsmuth, I.: Mathematische Fertigkeiten und mathematisches Verständnis. Bad Salzdetfurth 1985.

Weidenmann, B.: Psychische Prozesse beim Verstehen von Bildern. Verlag Hans Huber, Bern 1994.

Weidenmann, B.: Multicodierung und Multimodalität im Lernprozess. In: Issing, L.. Klimsa, P. (Hrsg.): Information und Lernen mit Multimedia, 2. überarbeitete Auflage; Beltz Psychologie-Verlags-Union, Weinheim, Basel 1997, S. 65-84.

Wittenberg, A.I.: Vom Denken in Begriffen: Mathematik als Experiment des reinen Denkens. Basel, Birkhäuser 1968.

Wittmann, E.Ch.: Mathematisches Denken bei Vor- und Grundschulkindern. Braunschweig 1982.

Wittmann, E.CH.; Müller G.N.: Handbuch produktiver Rechenübungen Band 1 Klett 1990.

Wittmann, E.CH.; Müller G.N.: Das kleine Zahlenbuch. Band 1. Spielen und Zählen, Kallmeyer 2002.

Woodruf, G.; Premack, D.: Primitive (sic), mathematical concepts in the chimpanzee: Proportionality and numerosity. Nature 1981, 293, 568-570.

Winn, W.: $\quad$ Learning in Hyperspace, University of Washington, http://weber.u.washington.edu/ billwinn. 2001

Winter, H.: $\quad$ Praxishilfe Mathematik. Frankfurt 1996.

Wynn, K.: $\quad$ Children's understanding of counting. In: Cognition 36 1990, 155-193.

Wynn, K.: $\quad$ Evidence against empiricist accounts of the origins of numerical knowledge. In: Mind and Language 1992a, 7, 315-331.

Wynn, K.: Children's acquisition of the number words and the counting system. In: Cocnitive Psychology 1992b, 24, 220-251.

Wynn, K.: Origins of numerical knowledge. In: Mathematical Cognition 1 1995, 3560 .

Wynn, K.: Competence models of numerical development. In: Cognitive Development 12, 1997, 333-339.

Zopfi Ch.: $\quad$ Basisschule in Holland: Die Lust am Spielen und Lernen. In: Kindergarten $1997 / 12$ S. $16-21$.

zur Oeveste H.: Kognitive Entwicklung in Vor- und Grundschulalter Göttingen, Verlag für Psychologie 1987. 


\section{Anhang}

\subsection{Das Interview}

Um sich ein Bild zu machen, wie das Interview genau abgelaufen ist, was für Schwierigkeiten zu bewältigen waren und wie sich die Kinder verhalten haben, werde ich im Folgenden das Interview zweier Kinder dokumentieren. Die beiden Kinder sind gleichaltrig, Melinda (IV/85) ist leistungsstark und Murat (IV/35) ist eher leistungsschwach. Da die vorliegende Kompetenzerhebung des OTZ sehr auf Bilder beruht, findet nur eine bescheidene Kommunikation statt. Nichts desto Trotz ist die Wiedergabe eines vollständigen Interviews aufschlussreich, vor allem bei Gegenüberstellungen von verschiedenen Lösungsstrategien wie bei dem vorliegenden Beispiel.

\section{Vergleichskonzepte}

Aufgabe A1. Hier siehst du Pilze. Zeige auf den Pilz, der höher ist als diese Blume. [Ex. zeigt auf die Blume in dem Kasten oben links auf dem Blatt.] (Siehe auch Seite 105.)

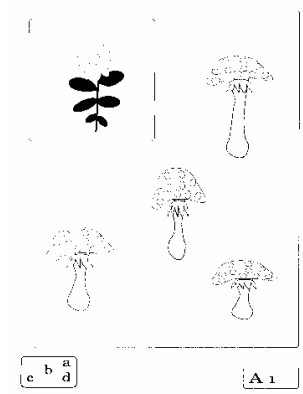

Melinda: Überlegt lange (12 sec.) und wirkt Murat: sehr unsicher. Der Interviewer (I)

Zeigt ohne zu zögern lächelnd auf den hilft ihr, indem er sie auffordert, erst mal alle Pilze zu zeigen. Melinda (M) legt ihre Anspannung ab und zeigt alle Pilz und zeigt dann nach Aufforderung des I langsam auf den höchsten Pilz. höchsten Pilz. 
Aufgabe A2. Hier siehst du Männer. Zeige auf den Mann, der dicker ist als dieser Mann. [Ex. zeigt auf den Mann in dem Kasten oben links auf dem Blatt.] (Siehe auch Seite 105.)

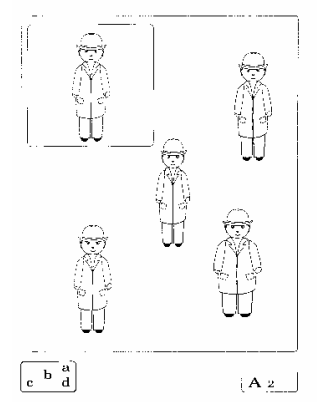

Melinda: Hier zeigt sie sofort und bestimmt $\mid$ Murat: Auch hier zeigt Murat (M) sofort auf auf den dicksten Mann. den dicksten Mann.

Aufgabe A3. Hier siehst du Gebäude. Zeige auf das niedrigste Gebäude. (Siehe auch Seite 106.)

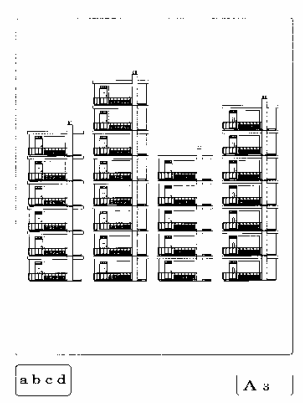

Melinda: Überlegt wieder lange (19s) und Murat: $\quad$ Er zeigt ohne Bedenken auf das höchsgibt dann zu verstehen, dass sie te Haus. nicht weiß, was das Wort „niedrigste" bedeutet.

Aufgabe A4. Hier siehst du Indianer. Zeige auf den Indianer, der weniger Federn hat als dieser Indianer mit Pfeil und Bogen. [Ex. zeigt auf den Indianer in dem Kasten oben links auf dem Blatt.] (Siehe auch Seite 106.) 


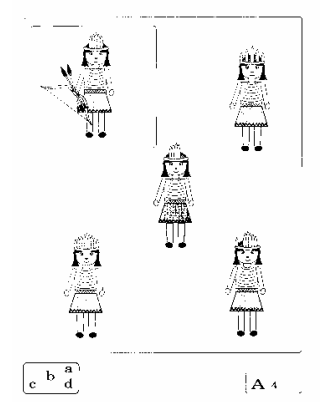

Melinda: Auch mit dem Wort „weniger“ weiß sie nichts anzufangen. Sie überlegt wieder lange (10s). I.: „Du darfst auch zählen wenn du willst." Darauf zeigt M. auf den falschen Indianer (a) mit der Bemerkung: „Ich hab nicht zählen müssen. “
Murat: Zeigt sofort und ohne zu zählen auf den richtigen Indianer in der Mitte des Bildes.

Aufgabe A5. Hier siehst du Kisten mit Murmeln. Zeige auf die Kiste mit den wenigsten Murmeln. (Siehe auch Seite 107.)

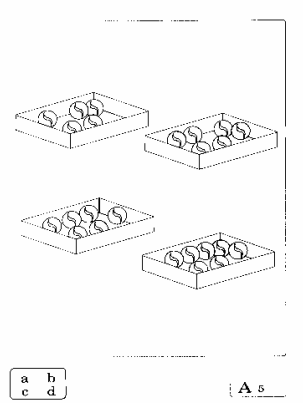

Melinda: Hier scheint sie mit dem Wort „wenigsten“ keine Mühe zu haben und zeigt sofort und ohne zu zählen auf die richtige Kiste.
Murat: Zeigt wieder sofort und ohne zu zählen auf die richtige Kiste. 


\section{Klassifizierung}

Aufgabe A6. Schaue dir diese Bilder an. Was kann nicht fliegen? (Siehe auch Seite 108.)

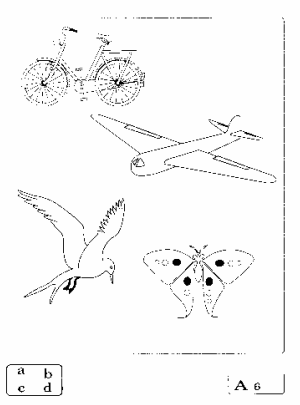

Melinda: Zeigt schnell auf das richtige Bild $\mid$ Murat: Zeigt schnell auf das richtige Bild. und lacht vor Vergnügen.

Aufgabe A7. Schaue dir diese Kästen an. Zeige auf den Kasten mit fünf Quadraten, aber ohne Dreieck. (Siehe auch Seite 108.)

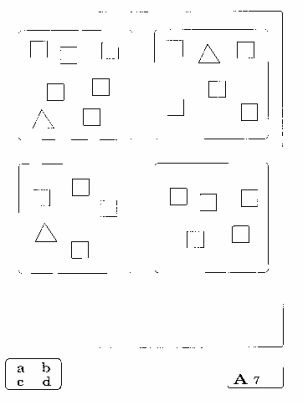

Melinda: Sofort und ohne zu zählen zeigt $\quad$ Murat: Zeigt ebenfalls sehr schnell und ohne sie das richtige Bild. zu zählen auf das richtige Bild.

Aufgabe A8. Schaue dir diese Bilder an. Zeige auf alle grauen Kreise. (Siehe auch Seite 109.)

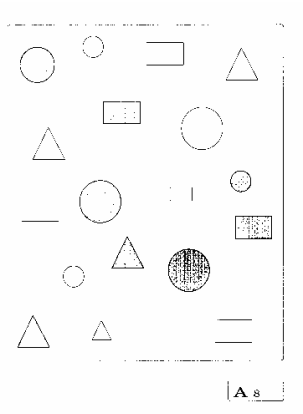

Melinda: Zeigt sofort die drei großen grau- $\mid$ Murat: Er zeigt zwei Rechtecke, ein Dreieck, 
en Kreise und übersieht den vierten kleinen, worauf der I. fragt:

"Hast du alle gezeigt?" Darauf zeigt M sofort auf den noch fehlenden kleinen. einen weißen und einen grauen Kreis.

(M kennt wohl die fundamentalen geometrischen Figuren nicht.)

Aufgabe A9. Hier siehst du Menschen. Zeige auf die Menschen, die eine Tasche, aber keine Brille tragen. (Siehe auch Seite 109.)

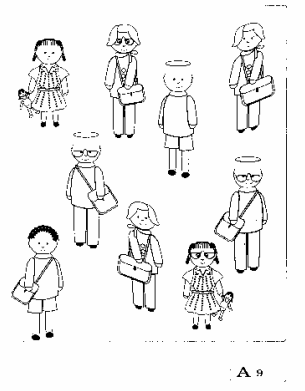

Melinda: Zeigt die richtigen Menschen. Sie $\mid$ Murat: $\quad$ Er zeigt zwei von drei richtigen Menbeginnt unten links und arbeitet schen sowie einen Menschen ohne sich systematisch durch das ganze Brille aber auch ohne Tasche. Bild.

Aufgabe A10. Hier siehst du einen Apfel mit Stiel, ohne Blatt und mit einem Würmchen, das aus dem Apfel herauskommt. [Ex. zeigt auf den Apfel in dem Kasten oben links auf dem Blatt.] Zeige auf alle Äpfel, die genau gleich aussehen. (Siehe auch Seite 110.)

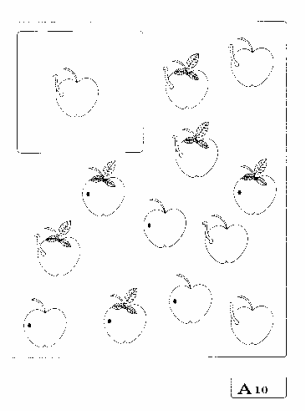

Melinda: Arbeitet wieder ganz exakt und findet ohne Schwierigkeiten die drei richtigen Äpfel. Sucht dann aber zur Sicherheit noch weitere,
Murat: $\quad$ Er zeigt alle Äpfel mit Stiel. Er fühlt sich sichtlich wohl und meint lächelnd zur Interviewerin: „Gell ich bin gut.“ 
findet keine mehr und sagt dann:

„So, ich habe jetzt alle.“

\section{Zuordnungen}

Aufgabe A11. [Ex. gibt dem Kind 10 Holzwürfel.] Du hast eine vier gewürfelt. [Ex. zeigt auf das Zahlbild mit der vier im Kasten.] Kannst du die gleiche Anzahl von Holzwürfeln auf den Tisch legen? (Siehe auch Seite 111.)

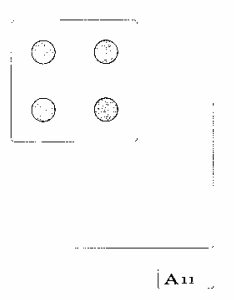

Melinda: Sie legt vier Holzwürfel exakt auf $\mid$ Murat: $\quad$ Er legt neben dem Vorlagenbild kordie vier Punkte des Würfelbildes rekt die gleiche Form. und sagt: ,Vier“.

Aufgabe A12. [Ex, gibt dem Kind 15 Holzwürfel.] Ich habe mit zwei Würfeln diese Zahlen gewürfelt. Kannst du die gleiche Anzahl von Holzwürfeln auf den Tisch legen? [Ex. zeigt auf die Zahlbilder mit der fünf und der sechs.] (Siehe auch Seite 112.)

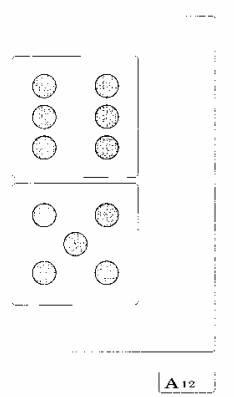

Melinda: Sie nimmt das Aufgabenblatt wahr und sagt sofort ohne zu zählen: „Fünf und sechs.“ Für die Lösung der Aufgabe legt sie diesmal die Holzwürfelchen neben der Vorlage in exakt dieser
Murat: Er legt wieder neben der Vorlage exakt die gleiche Form. 
Form. Der I. fragt: “Weißt du auch wie viele Würfelchen es sind?“" M. nach kurzer Betrachtung der Holzwürfelchen und ohne erkennbares zählen: „11“.

Aufgabe A13. [Ex. gibt dem Kind ein Arbeitsblatt und einen Bleistift.] Hier siehst du Kerzenhalter und Kerzen. In jeden Kerzenhalter sollen Kerzen gesteckt werden. Zeichne Linien von den Kerzen zu den passenden Kerzenhaltern. (Siehe auch Seite 113.)

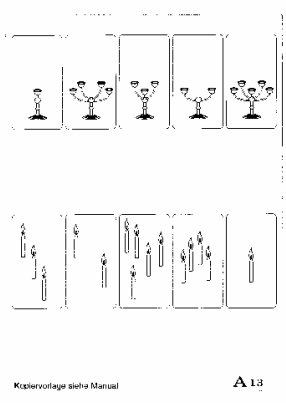

Melinda: Arbeitet die Kerzenhalter von links nach rechts korrekt ab, indem sie die richtigen Kerzenbilder zuordnet.
Murat: M zählt die Kerzenhalter im zweiten Kerzenhalterbild: „Vier“. Darauf zählt er die Kerzen im ersten Bild der zweiten Reihe: „Drei“ und schüttelt verneinend den Kopf. Er ordnet darauf die Bilder mit einer Kerze und dem entsprechendem Kerzenhalter zu. Er zählt dann wieder die einzelnen Bilder aus und ordnet auf Grund der Zählergebnisse alle Bilder korrekt einander zu.

Aufgabe A14. [Ex. gibt dem Kind ein Arbeitsblatt und einen Bleistift.] Hier siehst $d u$ drei Bilder mit Hühnern und Eiern. Kannst du das Bild finden, in dem jedes Huhn jeweils ein Ei gelegt hat? Du darfst Linien zeichnen. (Siehe auch Seite 113.) 


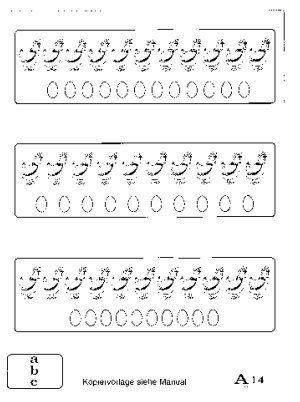

Melinda: Überlegt lange und beginnt am oberen Bild Zuordnungsstriche Huhn-Ei zu zeichnen. Es bleibt ein Ei übrig. Sie interpretiert dieses Ergebnis nicht verbal, sondern macht dasselbe beim mittleren Bild und erkennt, dass die Zuordnung aufgeht und streckt vor Freude die Arme hoch.
Murat: Er zeigt auf die je zwei Hühner der unteren Reihe, unter denen keine Eier liegen. Die I wiederholt die Frage und M zeigt jetzt auf das obere Bild und beginnt unter den beiden überhängenden Hühnern ein Ei zu zeichnen. (M hat offenbar die Frage nicht verstanden.)

Aufgabe A15. Hier siehst du fünfzehn Luftballons. [Ex. zeigt auf die Luftballons in dem Kasten oben links auf dem Blatt.] Zeige auf den Kasten, in dem genauso viele Punkte sind wie hier Luftballons. (Siehe auch Seite 114.)

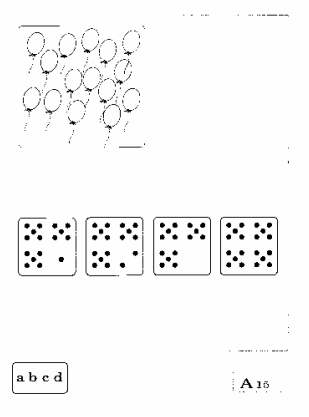

Melinda: Sie beginnt die Punkte des Kästchens unten links einzeln abzuzählen: „1, 2, 3, .., 15, 16“ und macht kommentarlos beim zweiten ebenso weiter: " $1,2, \ldots, 16$, $17^{\text {“ }}$ und beim dritten und findet so das richtige Kästchen.
Murat: Er beginnt sofort das Kästchen unten rechts auszuzählen und kommt auf nicht korrekte 14 Punkte und fragt dann: „Ist das richtig?““ 


\section{Reihenfolgen}

Aufgabe A16. Hier siehst du Kästen mit Äpfeln. Zeige auf den Kasten, in dem die Äpfel von groß nach klein geordnet sind. (Siehe auch Seite 115.)

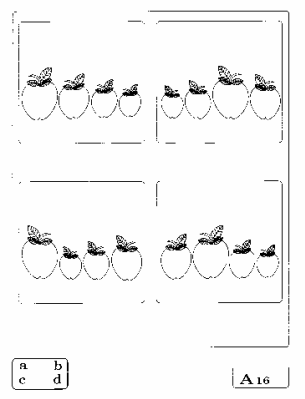

Melinda: Zeigt sofort auf das richtige Käst- $\mid$ Murat: $\quad$ M betrachtet die vier Kästchen nicht chen: „Da“. als Einheit, sondern er zeigt sehr frei von Kästchen zu Kästchen springend Äpfel in verschiedensten Größen.

Aufgabe A17. Hier siehst du Kästen mit Zuckerstangen. Zeige auf den Kasten, in dem die Zuckerstangen von dünn nach dick geordnet sind. (Siehe auch Seite 116.)

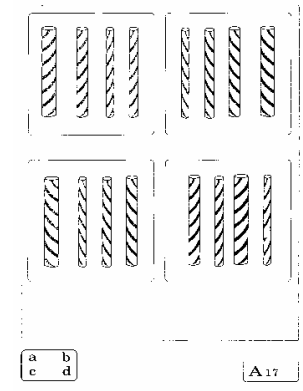

Melinda: Auch hier zeigt sie eben so sicher Murat: Auch bei dieser Aufgabe ist M offenund bestimmt wie vorhin auf das bar überfordert. Er hat sichtlich Mühe richtige Kästchen. mit der Begriffskonstruktion ,,von ... nach“

Aufgabe A18. Hier siehst du Kästen mit Murmeln. Zeige auf den Kasten, in dem die Murmeln von klein und hell nach groß und dunkel geordnet sind. (Siehe auch Seite 116.) 


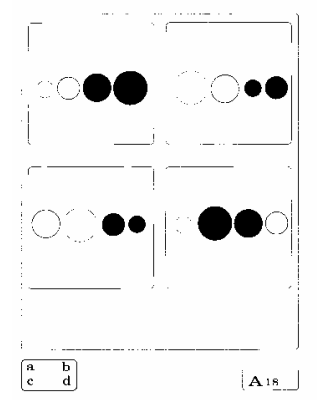

Melinda: Zeigt zuerst auf das falsche Käst- $\mid$ Murat: Er berührt ausnahmslos alle Murmeln. chen $\mathrm{c}$ und bemerkt den Fehler sofort mit der Bemerkung: „Äh, falsch."Und zeigt dann sofort auf das richtige Bild.

Aufgabe A19. [Ex. gibt dem Kind ein Arbeitsblatt und einen Bleistift.] Hier siehst du Hunde. Jeder Hund soll einen Stock bekommen. Ein großer Hund bekommt einen großen Stock und ein kleiner Hund bekommt einen kleinen Stock. Zeichne Linien von den Hunden zu den Stöcken, die sie bekommen. (Siehe auch Seite 117.)

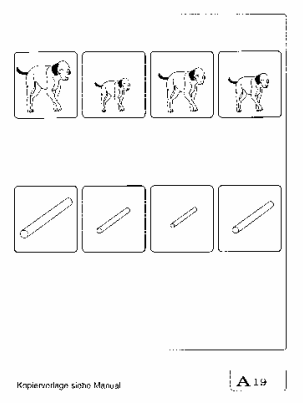

Melinda: Sie beginnt wieder systematisch mit dem linken Hund und arbeitet die übrigen in Leserichtung korrekt ab, indem sie die korrekten Zuordnungsstriche zeichnet und lehnt sich danach zufrieden zurück.
Murat: Er zeichnet Linien von den unteren Bildern zu den unmittelbar darüber liegenden Bildern und ist sichtlich stolz auf seine Leistung.

Aufgabe A20. Hier siehst du Stapel mit Brotscheiben in einer Reihe mit immer weniger Scheiben. [Ex. zeigt auf die Reihe von Brotscheiben unten auf dem Blatt.] Dieser Stapel mit Brotscheiben gehört irgendwo in die Reihe hinein. [Ex. zeigt auf die Brot- 
scheiben in dem Kasten oben links auf dem Blatt.] Zeige die Stelle, wo dieser Stapel in der Reihe hingehört. (Siehe auch Seite 117.)

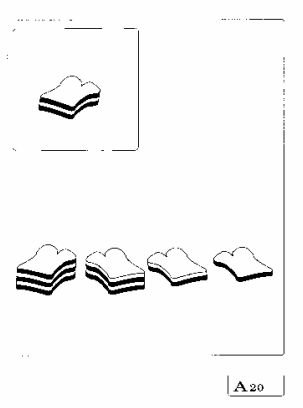

Melinda: Überlegt lange und der Interviewer erklärt dann die Form der Sandwichs, worauf M zaghaft und unsicher auf die richtige Stelle zeigt.
Murat: $\quad$ Er zeigt auf den niedrigsten Stapel rechts auf dem Bild ohne zu merken, dass er die Frage nicht beantwortet hat.

\section{Die Benutzung von Zahlwörtern}

Aufgabe A21. Zähle bis zwanzig. (Siehe auch Seite 120)

Melinda: Zählt sicher auf 20. „Ich kann noch weiter."
Murat: „1, 2, 3, 4, 5, 6, 7, 8, 9, 10, 11, 12, 13, $15,17,19,20$ “

Aufgabe A22. [Ex. zeigt dem Kind das Bild.] Zeige auf den Kasten mit sieben Punkten. (Siehe auch Seite 120.)
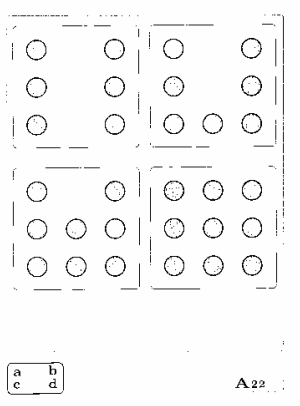

Melinda: Zeigt ohne erkennbares Zählen auf das richtige Bild. (Sie benutzt offenbar die vertraute Figur des Sechserwürfelbildes.)
Murat: Er zählt fehlerfrei alle Punkte in jedem Kästchen, zuletzt das mit sieben Punkten und zeigt dann triumphierend auf dieses Kästchen: „Sieben Punkte“. 
Aufgabe A23. Zähle weiter von neun bis fünfzehn: sechs, sieben, acht ... (Siehe auch Seite 121.)

Melinda: „,9, 10, 11, 12, 13, 14, 15“ Murat: „9, 1, 2, 3, 4, 5, 6, 7, 8, 9, 10, 11, 12, $13,14,16,18,20 “$. M wippt rhythmisch beim Zählprozess mit dem ganzen Körper. Interessanterweise nennt er jetzt im Gegensatz zur Aufgabe A21 nach der Zahl 13 die geraden Zahlen. M hat den Zahlraum bis 20 noch nicht erobert, ist aber sehr nahe dran.

Aufgabe A24. [Ex. zeigt dem Kind das Bild.] Zeige auf die achtzehnte Blume. (Siehe auch Seite 121.)

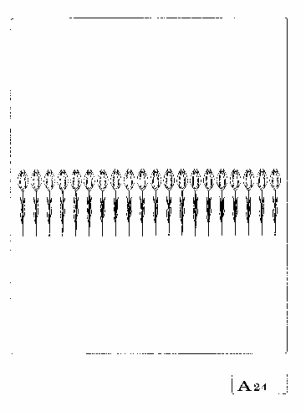

Melinda: Sie ist ratlos; denn sie hat die Aufgabe offenbar nicht verstanden. Dann aber scheint sie plötzlich zu begreifen und zählt die Blumen ab und zeigt mit dem Bleistift, den sie vor ihrem Gesicht in ihrer Hand hält, aus dieser Entfernung auf die gezählten Blumen, merkt, dass sie sich verzählt hat, beginnt wieder von vorne und verzählt sich abermals. Jetzt erst berührt sie mit ihrem Bleistift die gezählten Blumen
Murat: Er zählt die ersten acht Blumen ab, indem er mit dem Finger auf jede gezählte Blume zeigt. Die I wiederholt die Aufgabe, worauf M fragt: „Was muss ich machen?" und beginnt ohne auf eine Antwort zu warten von rechts die Blumen abzuzählen und hört dann bei neun auf. 
und kann so die gesuchte achtzehnte Blume zeigen.

Aufgabe A25. Zähle bis vierzehn und überspringe immer jeweils eine Zahl: zwei, vier, sechs ... (Siehe auch Seite 122.)

Melinda: „Oh, das kann ich nicht.“ Worauf $\mid$ Murat: „7, 8“, M hält fragend inne, wartet ein der I. die Frage und die Vorgabe paar Sekunden und beginnt dann zu wiederholt. M überlegt, versucht es: „,sieben, acht, ohh. Kann ich zählen: „,1, ..., 12, 13, 14, 15, 16, 17, nicht." und gibt auf.

$19,20 “$

\section{Strukturiertes Zählen}

Aufgabe A26. [Ex. legt 16 Holzwürfel in vier Reihen von jeweils vier Holzwürfeln mit etwas Abstand zwischen den Holzwürfeln auf den Tisch.] Zeige auf die Holzwürfel und zähle sie. (Siehe auch Seite 123.)

Melinda: Sie berührt mit ihrem Zeigefinger $\mid$ Murat: Er berührt mit seinem Zeigefinger die die zu zählenden Holzwürfel und zu zählenden Holzwürfel und zählt zählt laut bis 16 und nennt dann laut bis 16 .

diese Zählzahl als Ergebnis:

,sechzehn“.

Aufgabe A27. [Ex. legt neun Holzwürfel in einem Kreis mit etwas Abstand zwischen den Holzwürfeln auf den Tisch.] Zähle diese Holzwürfel. [Das Kind darf auf die Holzwürfel zeigen oder sie während des Zählens zur Seite schieben.] (Siehe auch Seite 124.) Melinda: Sie beginnt einfach darauf los zu $\mid$ Murat: Zählt ohne Probleme ganz korrekt und zählen. Bemerkt während des Zählprozesses, dass sie vergessen locker die neun Holzwürfelchen. $\mathrm{M}$ ist hat, wo sie begonnen hat und resehr selbstsicher und ruft einem vorbeigehendem Kameraden zu: „Hey, gistriert damit, dass sie die Anzahl ich habe noch keinen einzigen Fehler der Holzwürfelchen so nicht korgemacht."

rekt bestimmen kann. Sie beginnt 
daraufhin wieder von vorne, konzentriert sich aber auf den ersten gezählten Stein und weiß so am

Schluss des Zählprozesses, dass dieser erste Stein schon gezählt wurde und nennt so die letzte Zählzahl als Ergebnis: „Neun“.

Aufgabe A28. [Ex. legt 20 Holzwürfel in einem Haufen mit etwas Abstand zwischen den Holzwürfeln auf den Tisch.] Zähle diese Holzwürfel. [Das Kind darf auf die Holzwürfel zeigen oder sie während des Zählens zur Seite schieben.] (Siehe auch Seite 124.) Melinda: Sie berührt jeden Holzwürfel, schiebt den gezählten leicht beiMurat: Er zählt jedes Holzwürfelchen und seite und zählt dabei korrekt bis 20. berührt den gezählten Holzwürfel. Leider lässt er beim an sich korrekten Zählprozess die Zahl 14 aus und kommt so auf ein falsches Resultat.

Aufgabe A29. Ich gebe dir ein Bild, das du kurz anschauen sollst. [Ex. zeigt dem Kind das Bild für 2 Sekunden (zählen: ,einundzwanzig, zweiundzwanzig“) und nimmt das Bild weg.] Wie viele Punkte waren es zusammen? [Die Antwort „vier und fünf “ reicht aus.] (Siehe auch Seite 125.)

Melinda: „vier und fünf“

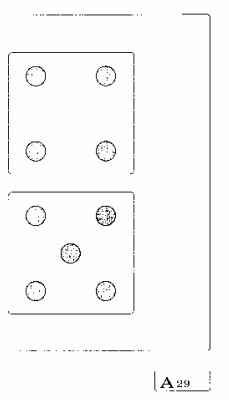

Murat: „13“"

Aufgabe A30. [Ex. legt 17 Holzwürfel auf den Tisch in einer Reihe mit etwas Abstand zwischen den Holzwürfeln.] Hier siehst du siebzehn Holzwürfel. Zeige auf die Holzwürfel und zähle sie rückwärts. (Siehe auch Seite 126.)

Melinda: Sie versteht zu Anfang das Rück- $\mid$ Murat: Auch M versteht das Rückwärtszählen 
wärtszählen geometrisch und zählt statt vorwärts in Leserichtung eben rückwärts von rechts nach links. Der I interveniert und erklärt das Rückwärtszählen, worauf $\mathrm{M}$ sich mit dieser für sie neuen Situation auseinandersetzt. Sie versucht: „,siebzehn, ... siebzehn, achtzehn, nein, siebzehn, ... oh kann ich nicht.“ geometrisch und zählt von rechts nach links von 1 an vorwärts. Die I lässt es dabei bewenden.

\section{Resultatives Zählen}

Aufgabe A31. [Ex. gibt dem Kind 15 Holzwürfel.] Lege eine Reihe mit elf Holzwürfeln. (Siehe auch Seite 128.)

Melinda: Sie legt die Holzwürfel stumm in eine Reihe vor sich hin und sagt dann: „Elf“".
Murat: $\quad$ Er schafft es, elf Holzwürfel aus dem Vorrat der 15 Holzwürfel zu isolieren, bildet aber keine Reihe, sondern legt sie strukturlos vor sich hin..

Aufgabe A32. [Ex. legt 20 Holzwürfel in einer Reihe auf den Tisch mit etwas Abstand zwischen den Holzwürfeln.] Wie viele Holzwürfel liegen hier? [Das Kind darf nicht auf die Holzwürfel zeigen.] (Siehe auch Seite 128.)

Melinda: Sie wippt beim stummen Zählpro- Murat: zess rhythmisch mit dem Kopf und nennt am Schluss korrekt: „Zwanzig“.
M möchte immer wieder mit den Fingern beim Zählen die Holzwürfelchen berühren. Die I unterbricht ihn aber jedes Mal und er beginnt von vorne. Nach einigen Malen hat er das Verbot des Berührens akzeptiert und er streckt jetzt bei jedem gezählten Holzwürfel einen Finger vor. Bei Zehn hört er auf; es sind keine Finger mehr da. 
Aufgabe A33. [Ex. legt 15 Holzwürfel auf den Tisch in drei Reihen mit jeweils fünf Holzwürfeln und mit etwas Abstand zwischen den Holzwürfeln.] Wie viele Holzwürfel liegen hier? [Das Kind darf nicht auf die Holzwürfel zeigen.] (Siehe auch Seite 128.)

Melinda: Sie zählt stumm, bewegt aber die Lippen beim rhythmischen Zählprozess und nennt auch hier korrekt: „fünfzehn“.
Murat: Er zählt jetzt anstelle der Holzwürfel seine Finger. Er streckt seine 10 Finger in die Höhe und sagt: ,,acht““. M ist müde.

Aufgabe A34. [Ex. legt 19 Holzwürfel in einem Haufen auf den Tisch mit etwas Abstand zwischen den Holzwürfeln.] Wie viele Holzwürfel liegen hier? [Das Kind darf nicht auf die Holzwürfel zeigen.] (Siehe auch Seite 129.)

Melinda: Stumm, aber auch hier wieder die $\mid$ Murat: Das Verbot des Berührens hat ihn sehr Lippen bewegend und mit dem irritiert und er zählt auch hier wieder Kopf rhythmisch wippend nennt seine Finger ab. Bei Zehn steckt er die sie ein wenig unsicher lächelnd Finger unter den Tisch und zählt bis die richtige Anzahl Holzwürfel: 12 weiter: ,Zwölf“. „neunzehn“.

Aufgabe A35. [Ex. legt 5 Holzwürfel auf den Tisch] Hier sind fünf Holzwürfel. Ich schiebe sie unter meine Hand. [Ex. schiebt die Holzwürfel unter seine Hand. Dann schiebt er 7 weitere Holzwürfel, die er dem Kind zeigt, unter seine Hand.] Ich füge sieben Holzwürfel dazu. Wie viele Holzwürfel habe ich jetzt unter meiner Hand? (Siehe auch Seite 129.)

Melinda: Überlegt lange (15s), schüttelt den Murat: „Vier und sieben“ Kopf, bedeckt dann ihr Gesicht mit ihren Händen und verharrt in dieser Stellung 30 Sekunden und streckt dann alle zehn Finger dem I entgegen. 


\section{Allgemeines Zahlenwissen}

Aufgabe A36. Hier siehst du zwei Kisten. [Ex. zeigt auf die Kisten in dem Bild.] Es sind neun Bonbons in der schwarzen Kiste. In der weißen Kiste sind dreizehn Bonbons. In welcher Kiste sind die meisten Bonbons? (Siehe auch Seite 131.)
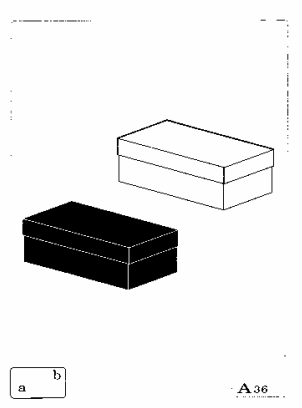

Melinda: Überlegt 7 Sekunden und zeigt $\quad$ Murat: Zeigt sofort auf die richtige Kiste. dann auf die richtige Kiste.

Aufgabe A37. [Ex. zeigt auf das Bild mit den 9 Murmeln.] Du hast neun Murmeln. Du verlierst drei Murmeln. Wie viele Murmeln hast du übrig? Zeige auf den Kasten mit der richtigen Anzahl von Murmeln. [Ex. zeigt auf die Reihe der Bilder unten auf dem Blatt.] (Siehe auch Seite 131.)

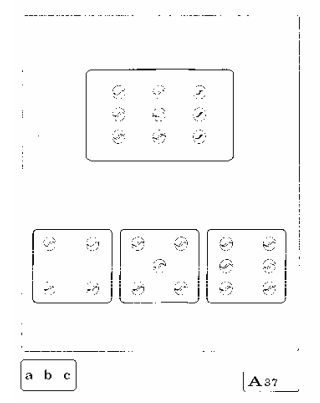

Melinda: Zeigt sofort auf das richtige Bild: $\mid$ Murat: Zählt alle Punkte in jedem Kästchen „Da“. Sie benutzt hier offensichtzwar korrekt ab, kann aber mit den licht die Struktur des SechserwürResultaten nichts anfangen. felbildes.

Aufgabe A38. [Ex. zeigt auf das Bild mit den 8 Hühnern.] Ein Bauer hat acht Hühner. Er kauft noch zwei Hühner. [Ex. zeigt auf das Bild mit den zwei Hühnern.] Wie viele 
Hühner hat der Bauer jetzt? Zeige auf den Kasten mit der richtigen Anzahl von Hühnern. [Ex. zeigt auf die Reihe von Bildern unten auf dem Blatt.] (Siehe auch Seite 132.)

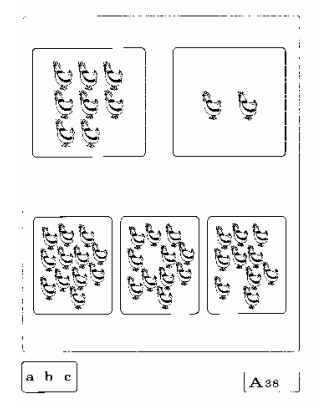

Melinda: „Ohho“ Sie sucht ohne Zählver- $\mid$ Murat: Zeigt auf das falsche mittlere Bild ohsuche das richtige Bild und zeigt ne zu zählen dann auf das falsche mittlere Bild.

Aufgabe A39. Hier siehst du ein Gebäude mit Fenster und Türen. [Ex. zeigt auf die Fenster und Türen in dem Gebäude, aber nicht auf jede(s) einzeln.] Bäume stehen auch vor dem Gebäude. Kannst du zählen wie viele Fenster das Gebäude hat? (Siehe auch Seite 133.)

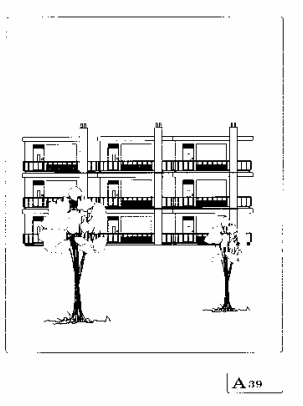

Melinda: Sie überlegt lange (15s) und zeigt $\mid$ Murat: Zählt ohne Probleme alle neun Fensdann auf den Baum, der ein Fenster. ter ganz verdeckt und fragt: „Den auch?“, worauf der I nickt. Sie überlegt wieder eine geraume Zeit (10s) und nennt dann korrekt: "neun".

Aufgabe A40. Du hast zweimal gewürfelt. [Ex. zeigt auf die beiden Würfelbilder] Welche Zahl hast du insgesamt gewürfelt und wohin darf die Figur gehen? (Siehe auch Seite 133.) 


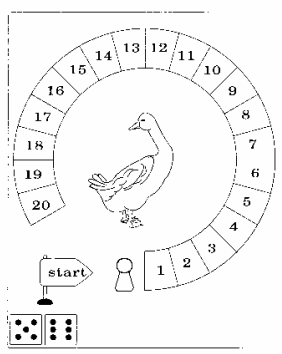

An
Melinda: Sie zeigt auf das korrekte Feld: „Da“.
Murat: Zählt einfach so gut es geht alle Felder des Spielplanes ab. Die I wiederholt die Frage und $\mathrm{M}$ besinnt sich, zählt alle Punkte der Würfelbilder: „elf“ und zeigt dann auf das richtige Feld. 


\subsection{Die Vierfeldertafel gespiegelt}

Man beachte, dass eine Umkehrung der Richtung keinen Einfluss auf den Trennschärfekoeffizienten ausübt.

\begin{tabular}{|c|c|c|c|}
\hline & + & - & $\sum$ \\
\hline$E$ & $a$ & $b$ & $a+b$ \\
\hline$M$ & $c$ & $d$ & $c+d$ \\
\hline$\sum$ & $a+c$ & $b+d$ & $\mathrm{~N}$ \\
\hline
\end{tabular}

$E$ : Erfolg; $M$ : Misserfolg; $N$ : alle Probanden

Der Trennschärfekoeffizient berechnet sich nach bekannter Formel:

$$
r=\frac{a d-b c}{\sqrt{(a+b)(c+d)(a+c)(b+d)}}(\text { Lienert/Raatz, 1998, 91) }
$$

Wechselt man nun die Richtung, sieht die Vierfeldertafel wie folgt aus:

\begin{tabular}{|c|c|c|c|}
\hline & $E$ & $M$ & $\sum$ \\
\hline+ & $a$ & $c$ & $a+c$ \\
\hline- & $b$ & $d$ & $b+d$ \\
\hline$\sum$ & $a+b$ & $c+d$ & $\mathrm{~N}$ \\
\hline
\end{tabular}

Man erkennt, dass nur die Felder $M+$ und $E-$ ihren Inhalt austauschen. Für die Berechnung des Trennschärfekoeffizienten hat das aber auf Grund der Gültigkeit des Kommutativgesetzes bezüglich der Multiplikation keinen Einfluss:

$$
r=\frac{a d-c b}{\sqrt{(a+c)(b+d)(a+b)(c+d)}}
$$


14.3 Vierfeldertafeln Alter und allgemeine Leistungsfähigkeit

Die Beziehungen

Alter $\rightarrow$ Vergleichkonzepte

\begin{tabular}{|l|l|l|l|}
\hline & + & - & $\sum$ \\
\hline $2 . \mathrm{KgJ}$ & 21 & 16 & 37 \\
\hline $1 . \mathrm{KgJ}$ & 16 & 17 & 33 \\
\hline$\sum$ & 37 & 33 & 70 \\
\hline
\end{tabular}

Korrelationskoeffizient $r=0.08$

Alter $\rightarrow$ Klassifizierungen

Korrelationskoeffizient $r=0.14$

\begin{tabular}{|l|c|c|c|}
\hline & + & - & $\sum$ \\
\hline $2 . \mathrm{KgJ}$ & 24 & 13 & 37 \\
\hline $1 . \mathrm{KgJ}$ & 17 & 16 & 33 \\
\hline$\sum$ & 41 & 29 & 70 \\
\hline
\end{tabular}

Alter $\rightarrow$ Eins-zu-eins-Zuordnungen

Korrelationskoeffizient $r=0.43$

\begin{tabular}{|l|c|c|c|}
\hline & + & - & $\sum$ \\
\hline $2 . \mathrm{KgJ}$ & 18 & 19 & 37 \\
\hline $1 . \mathrm{KgJ}$ & 3 & 30 & 33 \\
\hline$\sum$ & 21 & 49 & 70 \\
\hline
\end{tabular}

Alter $\rightarrow$ Reihenfolgen

Korrelationskoeffizient $r=0.43$

\begin{tabular}{|l|c|c|c|}
\hline & + & - & $\sum$ \\
\hline $2 . \mathrm{KgJ}$ & 21 & 16 & 37 \\
\hline $1 . \mathrm{KgJ}$ & 5 & 28 & 33 \\
\hline$\sum$ & 26 & 28 & 70 \\
\hline
\end{tabular}


Leistungsfähigkeit $\rightarrow$ Vergleichkonzepte

Korrelationskoeffizient $r=0.43$

\begin{tabular}{|l|c|c|c|}
\hline & + & - & $\sum$ \\
\hline Erfolgreich & 26 & 9 & 35 \\
\hline Nicht erfolgreich & 11 & 24 & 35 \\
\hline$\sum$ & 37 & 33 & 70 \\
\hline
\end{tabular}

Leistungsfähigkeit $\rightarrow$ Klassifizierungen

Korrelationskoeffizient $r=0.55$

\begin{tabular}{|l|c|c|c|}
\hline & + & - & $\sum$ \\
\hline Erfolgreich & 30 & 5 & 35 \\
\hline Nicht erfolgreich & 11 & 24 & 35 \\
\hline$\sum$ & 41 & 29 & 70 \\
\hline
\end{tabular}

Leistungsfähigkeit $\rightarrow$ Eins-zu-eins-Zuordnung

Korrelationskoeffizient $r=0.53$

\begin{tabular}{|l|c|c|c|}
\hline & + & - & $\sum$ \\
\hline Erfolgreich & 19 & 16 & 35 \\
\hline Nicht erfolgreich & 2 & 33 & 35 \\
\hline$\sum$ & 21 & 49 & 70 \\
\hline
\end{tabular}

Leistungsfähigkeit $\rightarrow$ Reihenfolgen

\begin{tabular}{|l|c|c|c|}
\hline & + & - & $\sum$ \\
\hline Erfolgreich & 24 & 11 & 35 \\
\hline Nicht erfolgreich & 2 & 33 & 35 \\
\hline$\sum$ & 21 & 49 & 70 \\
\hline
\end{tabular}

Korrelationskoeffizient $r=0.65$ 


\subsection{Ergebnisstabellen des „Item-Muster“" Verfahrens}

Gruppe GI:

\begin{tabular}{|l|c|c|c|c|c|c|c|c|c|c|c|c|c|}
\hline Kind & AG & Punkte & $\mathbf{1 0}$ & $\mathbf{1 3}$ & $\mathbf{1 4}$ & $\mathbf{1 9}$ & $\mathbf{2 1}$ & $\mathbf{2 8}$ & $\mathbf{3 1}$ & $\mathbf{2 3}$ & $\mathbf{2 5}$ & $\mathbf{3 7}$ & $\mathbf{3 0}$ \\
\hline Phillip & V & 95 & 1 & 1 & 1 & 1 & 1 & 1 & 1 & 1 & 1 & 1 & 1 \\
\hline Svenja & IV & 87.5 & 1 & 1 & 1 & 1 & 1 & 1 & & 1 & 1 & 1 & 1 \\
\hline Kimon & V & 87.5 & 1 & 1 & 1 & 1 & 1 & 1 & 1 & 1 & 1 & 1 & 1 \\
\hline Michael & IV & 87.5 & & 1 & 1 & 1 & 1 & 1 & 1 & 1 & 1 & 1 & 1 \\
\hline Melida & IV & 85 & 1 & 1 & 1 & 1 & 1 & 1 & 1 & 1 & & 1 & \\
\hline Daniela & V & 85 & 1 & 1 & 1 & 1 & 1 & 1 & 1 & & 1 & 1 & \\
\hline Ilona & I & 82.5 & 1 & 1 & 1 & & 1 & 1 & 1 & 1 & 1 & 1 & \\
\hline Florian & II & 82.5 & 1 & 1 & 1 & 1 & 1 & & 1 & 1 & 1 & 1 & 1 \\
\hline Haci & V & 82.5 & 1 & 1 & 1 & 1 & 1 & & & 1 & & 1 & 1 \\
\hline Silvan & V & 82.5 & 1 & 1 & 1 & 1 & 1 & 1 & & 1 & 1 & 1 & 1 \\
\hline Sabine & IV & 77.5 & & 1 & 1 & 1 & 1 & 1 & & 1 & & 1 & \\
\hline Kevin & V & 75 & 1 & 1 & 1 & & 1 & 1 & 1 & 1 & & & 1 \\
\hline Julien & III & 75 & 1 & 1 & & 1 & 1 & 1 & & & 1 & 1 & \\
\hline Adrian & IV & 75 & 1 & 1 & & 1 & 1 & 1 & & 1 & & 1 & \\
\hline Silvana & IV & 72.5 & 1 & 1 & 1 & 1 & 1 & 1 & 1 & 1 & & 1 & \\
\hline Miriam & IV & 72.5 & 1 & 1 & 1 & 1 & 1 & 1 & 1 & & 1 & 1 & \\
\hline Noemi & IV & 70 & 1 & & & 1 & & 1 & 1 & 1 & 1 & & 1 \\
\hline Veronika & IV & 70 & 1 & 1 & 1 & 1 & 1 & 1 & 1 & 1 & & 1 & \\
\hline Anna & IV & 65 & & 1 & & 1 & 1 & & & & & 1 & \\
\hline Meral & IV & 62.5 & 1 & & & & 1 & & 1 & 1 & 1 & 1 & \\
\hline Yanick & IV & 60 & & 1 & 1 & & 1 & 1 & 1 & 1 & & 1 & \\
\hline Nadine & IV & 60 & 1 & 1 & & & 1 & 1 & & & 1 & 1 & \\
\hline Evelyne & II & 60 & & 1 & 1 & & & 1 & 1 & & & 1 & \\
\hline Mathias & IV & 60 & & & & & 1 & & & 1 & & 1 & 1 \\
\hline Jasmin & II & 60 & 1 & 1 & & 1 & 1 & 1 & 1 & 1 & & & \\
\hline Alexandra & II & 60 & 1 & 1 & & & 1 & & 1 & & & 1 & \\
\hline Durchschnitt & & & 76.9 & 88.5 & 65.4 & 69.2 & 92.3 & 76.9 & 65.4 & 73.1 & 50.0 & 88.5 & 38.5 \\
\hline
\end{tabular}


Gruppe GII:

\begin{tabular}{|l|c|c|c|c|c|c|c|c|c|c|c|c|c|}
\hline Kind & AG & Punkte & $\mathbf{1 0}$ & $\mathbf{1 3}$ & $\mathbf{1 4}$ & $\mathbf{1 9}$ & $\mathbf{2 1}$ & $\mathbf{2 8}$ & $\mathbf{3 1}$ & $\mathbf{2 3}$ & $\mathbf{2 5}$ & $\mathbf{3 7}$ & $\mathbf{3 0}$ \\
\hline Sarina & I & 60 & 1 & 1 & 1 & 1 & & 1 & & 1 & & 1 & \\
\hline Andreas & V & 55 & 1 & 1 & & & 1 & 1 & & 1 & & & \\
\hline Sarah & VI & 55 & 1 & 1 & & 1 & 1 & & 1 & 1 & & 1 & \\
\hline Laurin & III & 55 & & 1 & & 1 & 1 & & 1 & & 1 & 1 & \\
\hline Daria & I & 55 & 1 & 1 & & 1 & & & 1 & & & & \\
\hline Debora & V & 52.5 & 1 & & 1 & & 1 & 1 & & & 1 & 1 & \\
\hline Melanie & III & 50 & & & & & 1 & 1 & 1 & 1 & & 1 & \\
\hline Alex & II & 50 & 1 & 1 & & 1 & & & & & & & \\
\hline Markus & I & 50 & & 1 & & & 1 & & 1 & & & & 1 \\
\hline Stefan & II & 47.5 & & 1 & & & & 1 & 1 & 1 & & 1 & \\
\hline Nouria & II & 47.5 & & 1 & 1 & & & & & & & & \\
\hline Marco & II & 47.5 & 1 & 1 & & & & 1 & 1 & 1 & & 1 & \\
\hline Melina & V & 45 & 1 & 1 & & 1 & & & & & & 1 & \\
\hline Mario & III & 45 & 1 & & & & 1 & 1 & 1 & 1 & & & \\
\hline Cédric & II & 45 & & & & & 1 & & 1 & 1 & & 1 & \\
\hline Sara & II & 42.5 & & & 1 & & 1 & 1 & & & & & \\
\hline Rebecca & II & 42.5 & 1 & 1 & 1 & 1 & & & & & & 1 & \\
\hline Cagri & I & 42.5 & 1 & 1 & & & 1 & & & 1 & & & \\
\hline Christoph & III & 42.5 & & 1 & 1 & & 1 & & 1 & 1 & & 1 & \\
\hline Ardita & III & 40 & 1 & 1 & & 1 & & & & & & 1 & \\
\hline Yannick & III & 40 & & & 1 & 1 & & & 1 & 1 & & & \\
\hline Claudia & II & 40 & & & & & 1 & 1 & 1 & & & & \\
\hline Vittorio & III & 37.5 & 1 & & & & 1 & & & & & 1 & \\
\hline Andres & II & 37.5 & 1 & 1 & & & & & & & & 1 & \\
\hline Samara & IV & 37.5 & & 1 & & 1 & & & & 1 & & & \\
\hline Kilian & V & 37.5 & & 1 & 1 & & & & & 1 & & 1 & \\
\hline Durchschnitt & & & 53.8 & 69.2 & 30.8 & 38.5 & 50.0 & 34.6 & 46.2 & 50.0 & 7.7 & 57.7 & 3.8 \\
\hline
\end{tabular}

Gruppe GIII:

\begin{tabular}{|l|c|c|c|c|c|c|c|c|c|c|c|c|c|}
\hline Kind & AG & Punkte & $\mathbf{1 0}$ & $\mathbf{1 3}$ & $\mathbf{1 4}$ & $\mathbf{1 9}$ & $\mathbf{2 1}$ & $\mathbf{2 8}$ & $\mathbf{3 1}$ & $\mathbf{2 3}$ & $\mathbf{2 5}$ & $\mathbf{3 7}$ & $\mathbf{3 0}$ \\
\hline Lorena & IV & 35 & 1 & & & & & & & & & & \\
\hline Miriam & II & 35 & 1 & & & 1 & & & & & & & \\
\hline Sami & IV & 35 & & & & & 1 & 1 & 1 & & & & \\
\hline Murat & IV & 35 & & 1 & & & & & 1 & & & & \\
\hline Christopher & II & 32.5 & & & & 1 & & & 1 & & & & \\
\hline Sascha & I & 32.5 & & & & 1 & & & & & & & \\
\hline Vera & I & 27.5 & 1 & & & & & & & & & & \\
\hline Tanja & III & 27.5 & & & & & & & & 1 & & & \\
\hline Ilenia & II & 27.5 & & & 1 & & & & & & & 1 & \\
\hline Roy & I & 25 & & & & & & & & & & & \\
\hline Yvonne & II & 25 & & & & & & & & & & & \\
\hline Christine & IV & 20 & 1 & & & & & & & & & & \\
\hline Jonas & III & 20 & & & & & & & & & & & \\
\hline Nico & II & 20 & & & & & & & & & & & \\
\hline Noah & II & 17.5 & & 1 & & & & & & & & & \\
\hline Michelle & II & 15 & & & & & & & & & & & \\
\hline Mark & I & 12.5 & & & & & & & & & & & \\
\hline Vanessa & II & 10 & & & & & & & & & & & \\
\hline Durchschnitt & & & 22.2 & 11.1 & 5.6 & 16.7 & 5.6 & 5.6 & 16.7 & 5.6 & 0.0 & 5.6 & 0.0 \\
\hline
\end{tabular}




\subsection{Alle Verknüpfungen des „Item-Muster“-Verfahrens}

$\mathrm{A10} \rightarrow \mathrm{Ann}$

\begin{tabular}{|c|c|c|c|c|c|}
\cline { 3 - 6 } \multicolumn{1}{c|}{} & GI & GII & GIII & Alle \\
\hline A10 $\rightarrow$ A13 & $1-1$ & 18 & 11 & 0 & 29 \\
\hline \multirow{4}{*}{ Trennschärfe } & $1-0$ & 2 & 3 & 4 & 9 \\
\cline { 2 - 6 } & $0-1$ & 5 & 7 & 2 & 14 \\
\cline { 2 - 6 } & $0-0$ & 1 & 5 & 12 & 18 \\
\cline { 2 - 6 } & & 0.09 & 0.22 & -0.19 & $\mathbf{0 . 3 3}$ \\
\hline
\end{tabular}

\begin{tabular}{|c|c|c|c|c|c|}
\cline { 3 - 6 } \multicolumn{2}{c|}{} & GI & GII & GIII & Alle \\
\hline A10 $\rightarrow$ A14 & $1-1$ & 13 & 3 & 0 & 16 \\
\hline \multirow{4}{*}{\begin{tabular}{c} 
Trennschärfe \\
\cline { 2 - 6 }
\end{tabular}} & $1-0$ & 7 & 11 & 4 & 22 \\
\cline { 2 - 6 } & $0-1$ & 4 & 5 & 1 & 10 \\
\cline { 2 - 6 } & & -0.01 & -0.22 & -0.13 & $\mathbf{0 . 1 1}$ \\
\hline
\end{tabular}

\begin{tabular}{|c|c|c|c|c|c|}
\cline { 3 - 6 } \multicolumn{1}{c|}{} & GI & GII & GIII & Alle \\
\hline A10 $\rightarrow$ A19 & $1-1$ & 15 & 7 & 1 & 23 \\
\hline \multirow{4}{*}{ Trennschärfe } & $1-0$ & 5 & 7 & 3 & 15 \\
\cline { 2 - 6 } & $0-1$ & 3 & 3 & 2 & 8 \\
\cline { 2 - 6 } & $0-0$ & 3 & 9 & 12 & 24 \\
\cline { 2 - 6 } & & 0.23 & 0.26 & 0.12 & $\mathbf{0 . 3 6}$ \\
\hline
\end{tabular}

\begin{tabular}{|c|c|c|c|c|c|}
\cline { 3 - 6 } \multicolumn{2}{c|}{} & GI & GII & GIII & Alle \\
\hline A10 $\rightarrow$ A21 & $1-1$ & 19 & 6 & 0 & 25 \\
\hline \multirow{4}{*}{\begin{tabular}{c} 
Trennschärfe \\
\cline { 2 - 6 }
\end{tabular}} & $1-0$ & 1 & 8 & 4 & 13 \\
\cline { 2 - 6 } & $0-1$ & 5 & 7 & 1 & 13 \\
\cline { 2 - 6 } & $0-0$ & 1 & 5 & 13 & 19 \\
\cline { 2 - 6 } & 0.18 & -0.15 & -0.13 & $\mathbf{0 . 2 5}$ \\
\hline
\end{tabular}

\begin{tabular}{|c|c|c|c|c|c|}
\cline { 3 - 6 } \multicolumn{2}{c|}{} & GI & GII & GIII & Alle \\
\hline A10 $\rightarrow$ A28 & $1-1$ & 16 & 5 & 0 & 21 \\
\hline \multirow{4}{*}{ Trennschärfe } & $1-0$ & 4 & 9 & 4 & 17 \\
\cline { 2 - 6 } & $0-1$ & 4 & 4 & 1 & 9 \\
\cline { 2 - 6 } & $0-0$ & 2 & 8 & 13 & 23 \\
\hline
\end{tabular}

\begin{tabular}{|c|c|c|c|c|c|}
\cline { 3 - 6 } \multicolumn{2}{c|}{} & GI & GII & GIII & Alle \\
\hline A10 $\rightarrow$ A31 & $1-1$ & 14 & 4 & 0 & 18 \\
\hline \multirow{4}{*}{ Trennschärfe } & $1-0$ & 6 & 10 & 4 & 20 \\
\cline { 2 - 6 } & $0-1$ & 3 & 8 & 3 & 14 \\
\cline { 2 - 6 } & $0-0$ & 3 & 4 & 11 & 18 \\
\hline
\end{tabular}

\begin{tabular}{|c|c|c|c|c|c|}
\cline { 3 - 6 } \multicolumn{2}{c|}{} & GI & GII & GIII & Alle \\
\hline A10 $\rightarrow$ A23 & $1-1$ & 15 & 6 & 0 & 21 \\
\hline \multirow{4}{*}{\begin{tabular}{c} 
Trennschärfe \\
\cline { 2 - 6 }
\end{tabular}} & $1-0$ & 5 & 8 & 4 & 17 \\
\cline { 2 - 6 } & $0-1$ & 4 & 7 & 1 & 12 \\
\cline { 2 - 6 } & $0-0$ & 2 & 5 & 13 & 20 \\
\cline { 2 - 6 } & & 0.08 & -0.15 & -0.13 & $\mathbf{0 . 1 8}$ \\
\hline
\end{tabular}

\begin{tabular}{|c|c|c|c|c|c|}
\cline { 2 - 6 } \multicolumn{2}{c|}{} & GI & GII & GIII & Alle \\
\hline A10 $\rightarrow$ A25 & $1-1$ & 12 & 1 & 0 & 13 \\
\hline \multirow{4}{*}{\begin{tabular}{c} 
Trennschärfe \\
\cline { 2 - 6 }
\end{tabular}} & $1-0$ & 8 & 13 & 4 & 25 \\
\cline { 2 - 6 } & $0-1$ & 1 & 1 & 0 & 30 \\
\cline { 2 - 6 } & $0-0$ & 5 & 11 & 14 & 22 \\
\hline
\end{tabular}

\begin{tabular}{|c|c|c|c|c|c|}
\cline { 3 - 6 } \multicolumn{2}{c|}{} & GI & GII & GIII & Alle \\
\hline A10 $\rightarrow$ A37 & $1-1$ & 18 & 9 & 0 & 26 \\
\hline \multirow{4}{*}{\begin{tabular}{c} 
Trennschärfe \\
\cline { 2 - 6 }
\end{tabular}} & $1-0$ & 3 & 5 & 4 & 12 \\
\cline { 2 - 6 } & $0-1$ & 6 & 6 & 1 & 13 \\
\cline { 2 - 6 } & $0-0$ & 0 & 6 & 13 & 19 \\
\cline { 2 - 6 } & & -0.20 & 0.14 & -0.13 & $\mathbf{0 . 2 8}$ \\
\hline
\end{tabular}

\begin{tabular}{|c|c|c|c|c|c|}
\cline { 2 - 6 } \multicolumn{2}{c|}{} & GI & GII & GIII & Alle \\
\hline A10 $\rightarrow$ A30 & $1-1$ & 8 & 0 & 0 & 8 \\
\hline \multirow{4}{*}{\begin{tabular}{c} 
Trennschärfe \\
\cline { 2 - 6 }
\end{tabular}} & $1-0$ & 12 & 14 & 4 & 30 \\
\cline { 2 - 6 } & $0-1$ & 2 & 1 & 0 & 3 \\
\cline { 2 - 6 } & $0-0$ & 4 & 11 & 14 & 29 \\
\hline
\end{tabular}

${ }^{68}$ Nicht definiert (Division durch Null). 
$\mathrm{A} 13 \rightarrow \mathrm{Ann}$

\begin{tabular}{|c|c|c|c|c|c|}
\cline { 3 - 6 } \multicolumn{1}{c|}{} & GI & GII & GIII & Alle \\
\hline A13 $\rightarrow$ A14 & $1-1$ & 17 & 5 & 0 & 22 \\
\hline \multirow{4}{*}{ Trennschärfe } & $1-0$ & 6 & 13 & 2 & 21 \\
\cline { 2 - 6 } & $0-1$ & 0 & 3 & 1 & 4 \\
\cline { 2 - 6 } & $0-0$ & 3 & 5 & 15 & 23 \\
\cline { 2 - 6 } & & 0.50 & -0.10 & -0.09 & $\mathbf{0 . 3 7}$ \\
\hline
\end{tabular}

\begin{tabular}{|c|c|c|c|c|c|}
\cline { 2 - 6 } \multicolumn{2}{c|}{} & GI & GII & GIII & Alle \\
\hline A13 $\rightarrow$ A19 & $1-1$ & 17 & 9 & 0 & 26 \\
\hline \multirow{4}{*}{\begin{tabular}{c} 
Trennschärfe \\
\cline { 2 - 6 }
\end{tabular}} & $1-0$ & 6 & 9 & 2 & 17 \\
\cline { 2 - 6 } & $0-1$ & 1 & 1 & 3 & 5 \\
\cline { 2 - 6 } & & 0.28 & 7 & 13 & 22 \\
\hline
\end{tabular}

\begin{tabular}{|c|c|c|c|c|c|}
\cline { 3 - 6 } \multicolumn{1}{c|}{} & GI & GII & GIII & Alle \\
\hline A13 $\rightarrow$ A21 & $1-1$ & 22 & 6 & 0 & 28 \\
\hline \multirow{4}{*}{ Trennschärfe } & $1-0$ & 1 & 12 & 2 & 15 \\
\cline { 2 - 6 } & $0-1$ & 2 & 7 & 1 & 10 \\
\cline { 2 - 6 } & $0-0$ & 1 & 1 & 15 & 17 \\
\cline { 2 - 6 } & & 0.35 & -0.50 & -0.09 & $\mathbf{0 . 2 7}$ \\
\hline
\end{tabular}

\begin{tabular}{|c|c|c|c|c|c|}
\cline { 3 - 6 } \multicolumn{2}{c|}{} & GI & GII & GIII & Alle \\
\hline A13 $\rightarrow$ A28 & $1-1$ & 19 & 4 & 0 & 23 \\
\hline \multirow{4}{*}{\begin{tabular}{c} 
Trennschärfe \\
\cline { 2 - 6 }
\end{tabular}} & $1-0$ & 4 & 14 & 2 & 20 \\
\cline { 2 - 6 } & $0-1$ & 1 & 5 & 1 & 7 \\
\cline { 2 - 6 } & $0-0$ & 2 & 3 & 15 & 20 \\
\cline { 2 - 6 } & 0.37 & -0.39 & -0.09 & $\mathbf{0 . 2 2}$ \\
\hline
\end{tabular}

\begin{tabular}{|c|c|c|c|c|c|}
\cline { 3 - 6 } \multicolumn{1}{c|}{} & GI & GII & GIII & Alle \\
\hline A13 $\rightarrow$ A31 & $1-1$ & 15 & 7 & 1 & 23 \\
\hline \multirow{4}{*}{\begin{tabular}{c} 
Trennschärfe \\
\cline { 2 - 6 }
\end{tabular}} & $1-0$ & 8 & 11 & 1 & 20 \\
\cline { 2 - 6 } & $0-1$ & 2 & 5 & 2 & 9 \\
\cline { 2 - 6 } & $0-0$ & 1 & 3 & 14 & 18 \\
\cline { 2 - 6 } & -0.01 & -0.22 & 0.32 & $\mathbf{0 . 2 0}$ \\
\hline
\end{tabular}

\begin{tabular}{|c|c|c|c|c|c|}
\cline { 2 - 6 } \multicolumn{2}{c|}{} & GI & GII & GIII & Alle \\
\hline A13 $\rightarrow$ A23 & $1-1$ & 16 & 9 & 0 & 25 \\
\hline \multirow{4}{*}{\begin{tabular}{c} 
Trennschärfe \\
\cline { 2 - 6 }
\end{tabular}} & $1-0$ & 7 & 9 & 2 & 18 \\
\cline { 2 - 6 } & $0-1$ & 3 & 4 & 1 & 8 \\
\cline { 2 - 6 } & & 0 & 4 & 15 & 19 \\
\hline
\end{tabular}

\begin{tabular}{|c|c|c|c|c|c|}
\hline & GI & GII & GIII & Alle \\
\hline $\mathrm{A} 13 \rightarrow \mathrm{A} 25$ & $1-1$ & 11 & 1 & 0 & 12 \\
\hline & $1-0$ & 12 & 17 & 2 & 31 \\
\hline & $0-1$ & 2 & 1 & 0 & 3 \\
\hline & $0-0$ & 1 & 7 & 16 & 24 \\
\hline Trennschärfe & & -0.12 & -0.12 & n.d. & 0.20 \\
\hline
\end{tabular}

\begin{tabular}{|c|c|c|c|c|c|}
\cline { 2 - 6 } \multicolumn{2}{c|}{} & GI & GII & GIII & Alle \\
\hline A13 $\rightarrow$ A37 & $1-1$ & 21 & 11 & 0 & 32 \\
\hline \multirow{4}{*}{\begin{tabular}{c} 
Trennschärfe \\
\cline { 2 - 6 }
\end{tabular}} & $1-0$ & 2 & 7 & 2 & 11 \\
\cline { 2 - 6 } & $0-1$ & 2 & 4 & 1 & 7 \\
\hline
\end{tabular}

\begin{tabular}{|c|c|c|c|c|c|}
\cline { 3 - 6 } \multicolumn{2}{c|}{} & GI & GII & GIII & Alle \\
\hline A13 $\rightarrow$ A30 & $1-1$ & 8 & 1 & 0 & 9 \\
\hline \multirow{4}{*}{\begin{tabular}{c} 
Trennschärfe \\
\cline { 2 - 6 }
\end{tabular}} & $1-0$ & 15 & 17 & 2 & 34 \\
\cline { 2 - 6 } & $0-1$ & 2 & 0 & 0 & 2 \\
\cline { 2 - 6 } & $0-0$ & 1 & 8 & 16 & 25 \\
\cline { 2 - 6 } & & -0.21 & 0.13 & n.d. & $\mathbf{0 . 1 8}$ \\
\hline
\end{tabular}


$\mathrm{A} 14 \rightarrow$ Ann

\begin{tabular}{|c|c|c|c|c|c|}
\cline { 3 - 6 } \multicolumn{1}{c|}{} & GI & GII & GIII & Alle \\
\hline A14 $\rightarrow$ A19 & $1-1$ & 13 & 3 & 0 & 16 \\
\hline \multirow{4}{*}{ Trennschärfe } & $1-0$ & 4 & 5 & 1 & 10 \\
\cline { 2 - 6 } & $0-1$ & 5 & 7 & 3 & 15 \\
\cline { 2 - 6 } & $0-0$ & 4 & 11 & 14 & 29 \\
\cline { 2 - 6 } & & 0.22 & -0.01 & -0.11 & $\mathbf{0 . 2 7}$ \\
\hline
\end{tabular}

\begin{tabular}{|c|c|c|c|c|c|}
\cline { 2 - 6 } \multicolumn{2}{c|}{} & GI & GII & GIII & Alle \\
\hline A14 $\rightarrow$ A21 & $1-1$ & 16 & 3 & 0 & 19 \\
\hline \multirow{4}{*}{\begin{tabular}{c} 
Trennschärfe \\
\cline { 2 - 6 }
\end{tabular}} & $1-0$ & 1 & 5 & 1 & 7 \\
\cline { 2 - 6 } & $0-1$ & 8 & 10 & 1 & 19 \\
\cline { 2 - 6 } & & 0.09 & -0.17 & -0.06 & $\mathbf{0 . 2 9}$ \\
\hline
\end{tabular}

\begin{tabular}{|c|c|c|c|c|c|}
\cline { 3 - 6 } \multicolumn{1}{c|}{} & GI & GII & GIII & Alle \\
\hline A14 $\rightarrow$ A28 & $1-1$ & 15 & 3 & 0 & 18 \\
\hline \multirow{4}{*}{ Trennschärfe } & $1-0$ & 2 & 5 & 1 & 8 \\
\cline { 2 - 6 } & $0-1$ & 5 & 6 & 1 & 12 \\
\cline { 2 - 6 } & $0-0$ & 4 & 12 & 16 & 32 \\
\hline \multirow{2}{*}{ Tyyyyy} & 0.37 & 0.04 & -0.06 & $\mathbf{0 . 4 1}$ \\
\hline
\end{tabular}

\begin{tabular}{|c|c|c|c|c|c|}
\cline { 3 - 6 } \multicolumn{2}{c|}{} & GI & GII & GIII & Alle \\
\hline A14 $\rightarrow$ A31 & $1-1$ & 13 & 2 & 0 & 15 \\
\hline \multirow{4}{*}{\begin{tabular}{c} 
Trennschärfe \\
\cline { 2 - 6 }
\end{tabular}} & $1-0$ & 4 & 6 & 1 & 11 \\
\cline { 2 - 6 } & $0-1$ & 4 & 10 & 3 & 17 \\
\cline { 2 - 6 } & $0-0$ & 5 & 8 & 14 & 27 \\
\cline { 2 - 6 } & 0.32 & -0.28 & -0.11 & $\mathbf{0 . 1 8}$ \\
\hline
\end{tabular}

\begin{tabular}{|c|c|c|c|c|c|}
\cline { 3 - 6 } \multicolumn{1}{c|}{} & GI & GII & GIII & Alle \\
\hline A14 $\rightarrow$ A23 & $1-1$ & 14 & 4 & 0 & 18 \\
\hline \multirow{4}{*}{ Trennschärfe } & $1-0$ & 3 & 4 & 1 & 8 \\
\cline { 2 - 6 } & $0-1$ & 5 & 9 & 1 & 15 \\
\cline { 2 - 6 } & $0-0$ & 4 & 9 & 16 & 29 \\
\cline { 2 - 6 } & & 0.29 & 0.00 & -0.06 & $\mathbf{0 . 3 4}$ \\
\hline
\end{tabular}

\begin{tabular}{|c|c|c|c|c|c|}
\cline { 2 - 6 } \multicolumn{2}{c|}{} & GI & GII & GIII & Alle \\
\hline A14 $\rightarrow$ A25 & $1-1$ & 9 & 1 & 0 & 10 \\
\hline \multirow{4}{*}{\begin{tabular}{c} 
Trennschärfe \\
\cline { 2 - 6 }
\end{tabular}} & $1-0$ & 8 & 7 & 1 & 16 \\
\cline { 2 - 6 } & $0-1$ & 4 & 1 & 3 & 8 \\
\hline
\end{tabular}

\begin{tabular}{|c|c|c|c|c|c|}
\cline { 3 - 6 } \multicolumn{1}{c|}{} & GI & GII & GIII & Alle \\
\hline A14 $\rightarrow$ A37 & $1-1$ & 16 & 5 & 1 & 22 \\
\hline \multirow{4}{*}{ Trennschärfe } & $1-0$ & 1 & 3 & 0 & 4 \\
\cline { 2 - 6 } & $0-1$ & 7 & 10 & 0 & 17 \\
\cline { 2 - 6 } & $0-0$ & 2 & 8 & 17 & 27 \\
\cline { 2 - 6 } & & 0.24 & 0.06 & 1.00 & $\mathbf{0 . 4 5}$ \\
\hline
\end{tabular}

\begin{tabular}{|c|c|c|c|c|c|}
\cline { 2 - 6 } \multicolumn{2}{c|}{} & GI & GII & GIII & Alle \\
\hline A14 $\rightarrow$ A30 & $1-1$ & 8 & 0 & 0 & 8 \\
\hline \multirow{4}{*}{\begin{tabular}{c} 
Trennschärfe \\
\cline { 2 - 6 }
\end{tabular}} & $1-0$ & 9 & 8 & 1 & 18 \\
\cline { 2 - 6 } & $0-1$ & 2 & 1 & 0 & 3 \\
\hline
\end{tabular}


$\mathrm{A} 19 \rightarrow$ Ann

\begin{tabular}{|c|c|c|c|c|c|}
\cline { 3 - 6 } \multicolumn{1}{c|}{} & GI & GII & GIII & Alle \\
\hline A19 $\rightarrow$ A21 & $1-1$ & 17 & 2 & 0 & 19 \\
\hline \multirow{4}{*}{\begin{tabular}{c} 
Trennschärfe \\
\cline { 2 - 6 }
\end{tabular}} & $1-0$ & 1 & 8 & 3 & 12 \\
\cline { 2 - 6 } & $0-1$ & 7 & 11 & 1 & 19 \\
\cline { 2 - 6 } & $0-0$ & 1 & 5 & 14 & 20 \\
\cline { 2 - 6 } & & 0.12 & -0.47 & -0.11 & $\mathbf{0 . 1 3}$ \\
\hline
\end{tabular}

\begin{tabular}{|c|c|c|c|c|c|}
\cline { 2 - 6 } \multicolumn{2}{c|}{} & GI & GII & GIII & Alle \\
\hline A19 $\rightarrow$ A28 & $1-1$ & 15 & 1 & 0 & 16 \\
\hline \multirow{4}{*}{\begin{tabular}{c} 
Trennschärfe \\
\cline { 2 - 6 }
\end{tabular}} & $1-0$ & 3 & 9 & 3 & 15 \\
\cline { 2 - 6 } & $0-1$ & 5 & 8 & 1 & 14 \\
\cline { 2 - 6 } & & 3 & 8 & 14 & 25 \\
\hline
\end{tabular}

\begin{tabular}{|c|c|c|c|c|c|}
\cline { 3 - 6 } \multicolumn{1}{c|}{} & GI & GII & GIII & Alle \\
\hline A19 $\rightarrow$ A31 & $1-1$ & 11 & 4 & 1 & 16 \\
\hline \multirow{4}{*}{\begin{tabular}{c} 
Trennschärfe \\
\cline { 2 - 6 }
\end{tabular}} & $1-0$ & 7 & 6 & 2 & 15 \\
\cline { 2 - 6 } & $0-1$ & 6 & 8 & 2 & 16 \\
\cline { 2 - 6 } & $0-0$ & 2 & 8 & 13 & 23 \\
\cline { 2 - 6 } & -0.13 & -0.10 & 0.20 & $\mathbf{0 . 1 1}$ \\
\hline
\end{tabular}

\begin{tabular}{|c|c|c|c|c|c|}
\cline { 2 - 6 } \multicolumn{2}{c|}{} & GI & GII & GIII & Alle \\
\hline A19 $\rightarrow$ A23 & $1-1$ & 14 & 4 & 0 & 18 \\
\hline \multirow{4}{*}{\begin{tabular}{c} 
Trennschärfe \\
\cline { 2 - 6 }
\end{tabular}} & $1-0$ & 4 & 6 & 3 & 13 \\
\cline { 2 - 6 } & $0-1$ & 5 & 9 & 1 & 15 \\
\cline { 2 - 6 } & 0.0 & 3 & 7 & 14 & 24 \\
\cline { 2 - 6 } & 0.16 & -0.16 & -0.13 & $\mathbf{0 . 2 0}$ \\
\hline
\end{tabular}

\begin{tabular}{|c|c|c|c|c|c|}
\cline { 3 - 6 } \multicolumn{1}{c|}{} & GI & GII & GIII & Alle \\
\hline A19 $\rightarrow$ A25 & $1-1$ & 10 & 1 & 0 & 11 \\
\hline \multirow{4}{*}{ Trennschärfe } & $1-0$ & 8 & 9 & 3 & 20 \\
\cline { 2 - 6 } & $0-1$ & 3 & 1 & 0 & 4 \\
\cline { 2 - 6 } & $0-0$ & 5 & 15 & 15 & 35 \\
\cline { 2 - 6 } & & 0.17 & 0.07 & n.d. & $\mathbf{0 . 3 1}$ \\
\hline
\end{tabular}

\begin{tabular}{|c|c|c|c|c|c|}
\cline { 2 - 6 } \multicolumn{2}{c|}{} & GI & GII & GIII & Alle \\
\hline A19 $\rightarrow$ A37 & $1-1$ & 16 & 6 & 0 & 22 \\
\hline \multirow{4}{*}{\begin{tabular}{c} 
Trennschärfe \\
\cline { 2 - 6 }
\end{tabular}} & $1-0$ & 2 & 4 & 3 & 9 \\
\cline { 2 - 6 } & $0-1$ & 7 & 9 & 1 & 17 \\
\cline { 2 - 6 } & & 1 & 7 & 14 & 22 \\
\cline { 2 - 6 } & & & & &
\end{tabular}

\begin{tabular}{|c|c|c|c|c|c|}
\cline { 3 - 6 } \multicolumn{2}{c|}{} & GI & GII & GIII & Alle \\
\hline A19 $\rightarrow$ A30 & $1-1$ & 8 & 0 & 0 & 8 \\
\hline \multirow{4}{*}{ Trennschärfe } & $1-0$ & 10 & 10 & 3 & 23 \\
\cline { 2 - 6 } & $0-1$ & 2 & 1 & 0 & 3 \\
\cline { 2 - 6 } & $0-0$ & 6 & 15 & 15 & 36 \\
\hline
\end{tabular}


A21 $\rightarrow$ Ann

\begin{tabular}{|c|c|c|c|c|c|}
\cline { 3 - 6 } \multicolumn{1}{c|}{} & GI & GII & GIII & Alle \\
\hline A21 $\rightarrow$ A28 & $1-1$ & 18 & 6 & 1 & 25 \\
\hline \multirow{4}{*}{\begin{tabular}{c} 
Trennschärfe \\
\cline { 2 - 6 }
\end{tabular}} & $1-0$ & 6 & 7 & 0 & 13 \\
\cline { 2 - 6 } & $0-1$ & 2 & 3 & 0 & 5 \\
\cline { 2 - 6 } & $0-0$ & 0 & 10 & 17 & 27 \\
\cline { 2 - 6 } & & -0.16 & 0.24 & 1.00 & $\mathbf{0 . 5 0}$ \\
\hline
\end{tabular}

\begin{tabular}{|c|c|c|c|c|c|}
\cline { 3 - 6 } \multicolumn{2}{c|}{} & GI & GII & GIII & Alle \\
\hline A21 $\rightarrow$ A31 & $1-1$ & 15 & 8 & 1 & 24 \\
\hline \multirow{4}{*}{\begin{tabular}{c} 
Trennschärfe \\
\cline { 2 - 6 }
\end{tabular}} & $1-0$ & 9 & 4 & 0 & 13 \\
\cline { 2 - 6 } & $0-1$ & 2 & 4 & 2 & 8 \\
\cline { 2 - 6 } & $0-0$ & 0 & 10 & 15 & 25 \\
\hline
\end{tabular}

\begin{tabular}{|c|c|c|c|c|c|}
\cline { 3 - 6 } \multicolumn{1}{c|}{} & GI & GII & GIII & Alle \\
\hline A21 $\rightarrow$ A23 & $1-1$ & 18 & 7 & 0 & 25 \\
\hline \multirow{4}{*}{ Trennschärfe } & $1-0$ & 6 & 6 & 1 & 13 \\
\cline { 2 - 6 } & $0-1$ & 1 & 6 & 1 & 8 \\
\cline { 2 - 6 } & $0-0$ & 1 & 7 & 16 & 24 \\
\cline { 2 - 6 } & & 0.15 & 0.08 & -0.06 & $\mathbf{0 . 4 1}$ \\
\hline
\end{tabular}

\begin{tabular}{|c|c|c|c|c|c|}
\cline { 3 - 6 } \multicolumn{2}{c|}{} & GI & GII & GIII & Alle \\
\hline A21 $\rightarrow$ A25 & $1-1$ & 12 & 2 & 0 & 14 \\
\hline \multirow{4}{*}{\begin{tabular}{c} 
Trennschärfe \\
\cline { 2 - 6 }
\end{tabular}} & $1-0$ & 12 & 11 & 1 & 24 \\
\cline { 2 - 6 } & $0-1$ & 1 & 0 & 0 & 1 \\
\cline { 2 - 6 } & $0-0$ & 1 & 13 & 17 & 31 \\
\cline { 2 - 6 } & 0.00 & 0.29 & n.d. & $\mathbf{0 . 4 1}$ \\
\hline
\end{tabular}

\begin{tabular}{|c|c|c|c|c|c|}
\cline { 3 - 6 } \multicolumn{1}{c|}{} & GI & GII & GIII & Alle \\
\hline A21 $\rightarrow$ A37 & $1-1$ & 22 & 7 & 0 & 29 \\
\hline \multirow{4}{*}{ Trennschärfe } & $1-0$ & 2 & 6 & 1 & 9 \\
\cline { 2 - 6 } & $0-1$ & 1 & 8 & 1 & 10 \\
\cline { 2 - 6 } & $0-0$ & 1 & 5 & 16 & 22 \\
\cline { 2 - 6 } & & 0.35 & -0.08 & -0.06 & $\mathbf{0 . 4 5}$ \\
\hline
\end{tabular}

\begin{tabular}{|c|c|c|c|c|c|}
\cline { 2 - 6 } \multicolumn{2}{c|}{} & GI & GII & GIII & Alle \\
\hline A21 $\rightarrow$ A30 & $1-1$ & 9 & 1 & 0 & 10 \\
\hline \multirow{4}{*}{\begin{tabular}{c} 
Trennschärfe \\
\cline { 2 - 6 }
\end{tabular}} & $1-0$ & 15 & 12 & 1 & 28 \\
\cline { 2 - 6 } & $0-1$ & 1 & 0 & 0 & 1 \\
\hline
\end{tabular}

$\mathrm{A} 28 \rightarrow \mathrm{A} n n$

\begin{tabular}{|c|c|c|c|c|c|}
\cline { 3 - 6 } \multicolumn{1}{c|}{} & GI & GII & GIII & Alle \\
\hline $\mathrm{A} 28 \rightarrow \mathrm{A} 31$ & $1-1$ & 14 & 5 & 1 & 20 \\
\hline \multirow{4}{*}{\begin{tabular}{c} 
Trennschärfe \\
\cline { 2 - 6 }
\end{tabular}} & $1-0$ & 6 & 4 & 0 & 10 \\
\cline { 2 - 6 } & $0-1$ & 3 & 7 & 2 & 12 \\
\cline { 2 - 6 } & $0-0$ & 3 & 10 & 15 & 28 \\
\hline
\end{tabular}

\begin{tabular}{|c|c|c|c|c|c|}
\cline { 2 - 6 } \multicolumn{2}{c|}{} & GI & GII & GIII & Alle \\
\hline A28 $\rightarrow$ A23 & $1-1$ & 15 & 6 & 0 & 21 \\
\hline \multirow{4}{*}{\begin{tabular}{c} 
Trennschärfe \\
\cline { 2 - 6 }
\end{tabular}} & $1-0$ & 5 & 3 & 1 & 9 \\
\cline { 2 - 6 } & $0-1$ & 4 & 7 & 1 & 12 \\
\hline & $0-0$ & 2 & 10 & 16 & 28 \\
\hline
\end{tabular}

\begin{tabular}{|c|c|c|c|c|c|}
\hline & GI & GII & GIII & Alle \\
\hline $\mathrm{A} 28 \rightarrow \mathrm{A} 25$ & $1-1$ & 11 & 1 & 0 & 12 \\
\hline & $1-0$ & 9 & 8 & 1 & 18 \\
\hline & $0-1$ & 2 & 1 & 0 & 3 \\
\hline & $0-0$ & 4 & 16 & 17 & 37 \\
\hline Trennschärfe & & 0.18 & 0.09 & n.d. & 0.39 \\
\hline
\end{tabular}

\begin{tabular}{|c|c|c|c|c|c|}
\cline { 2 - 6 } \multicolumn{2}{c|}{} & GI & GII & GIII & Alle \\
\hline A28 $\rightarrow$ A25 & $1-1$ & 17 & 5 & 0 & 22 \\
\hline \multirow{4}{*}{\begin{tabular}{c} 
Trennschärfe \\
\cline { 2 - 6 }
\end{tabular}} & $1-0$ & 3 & 4 & 1 & 8 \\
\cline { 2 - 6 } & $0-1$ & 6 & 10 & 1 & 17 \\
\cline { 2 - 6 } & & 0 & 7 & 16 & 23 \\
\hline
\end{tabular}

\begin{tabular}{|c|c|c|c|c|c|}
\cline { 3 - 6 } \multicolumn{2}{c|}{} & GI & GII & GIII & Alle \\
\hline A28 $\rightarrow$ A30 & $1-1$ & 7 & 0 & 0 & 7 \\
\hline \multirow{4}{*}{\begin{tabular}{c} 
Trennschärfe \\
\cline { 2 - 6 }
\end{tabular}} & $1-0$ & 13 & 9 & 1 & 23 \\
\cline { 2 - 6 } & $0-1$ & 3 & 1 & 0 & 4 \\
\cline { 2 - 6 } & $0-0$ & 3 & 16 & 17 & 36 \\
\hline
\end{tabular}


A31 $\rightarrow$ Ann

\begin{tabular}{|c|c|c|c|c|c|}
\cline { 3 - 6 } \multicolumn{2}{c|}{} & GI & GII & GIII & Alle \\
\hline A31 $\rightarrow$ A23 & $1-1$ & 13 & 8 & 0 & 21 \\
\hline \multirow{4}{*}{ Trennschärfe } & $1-0$ & 4 & 4 & 3 & 11 \\
\cline { 2 - 6 } & $0-1$ & 6 & 5 & 1 & 12 \\
\cline { 2 - 6 } & $0-0$ & 3 & 9 & 14 & 26 \\
\hline & & 0.11 & 0.31 & -0.11 & $\mathbf{0 . 3 4}$ \\
\hline
\end{tabular}

\begin{tabular}{|c|c|c|c|c|c|}
\cline { 2 - 6 } \multicolumn{2}{c|}{} & GI & GII & GIII & Alle \\
\hline A31 $\rightarrow$ A125 & $1-1$ & 9 & 1 & 0 & 10 \\
\hline \multirow{4}{*}{\begin{tabular}{c} 
Trennschärfe \\
\cline { 2 - 6 }
\end{tabular}} & $1-0$ & 8 & 11 & 3 & 22 \\
\cline { 2 - 6 } & $0-1$ & 4 & 1 & 0 & 5 \\
\cline { 2 - 6 } & & 5 & 13 & 15 & 33 \\
\hline
\end{tabular}

\begin{tabular}{|c|c|c|c|c|c|}
\cline { 3 - 6 } \multicolumn{1}{c|}{} & GI & GII & GIII & Alle \\
\hline A31 $\rightarrow$ A37 & $1-1$ & 14 & 7 & 0 & 21 \\
\hline \multirow{4}{*}{ Trennschärfe } & $1-0$ & 3 & 5 & 3 & 11 \\
\cline { 2 - 6 } & $0-1$ & 9 & 8 & 1 & 18 \\
\cline { 2 - 6 } & $0-0$ & 0 & 6 & 14 & 20 \\
\cline { 2 - 6 } & & -0.26 & 0.01 & -0.11 & $\mathbf{0 . 1 8}$ \\
\hline
\end{tabular}

\begin{tabular}{|c|c|c|c|c|c|}
\cline { 3 - 6 } \multicolumn{2}{c|}{} & GI & GII & GIII & Alle \\
\hline A31 $\rightarrow$ A30 & $1-1$ & 6 & 1 & 0 & 7 \\
\hline \multirow{4}{*}{\begin{tabular}{c} 
Trennschärfe \\
\cline { 2 - 6 }
\end{tabular}} & $1-0$ & 11 & 11 & 3 & 25 \\
\cline { 2 - 6 } & $0-1$ & 4 & 0 & 0 & 4 \\
\cline { 2 - 6 } & $0-0$ & 5 & 14 & 15 & 34 \\
\cline { 2 - 6 } & -0.09 & 0.22 & n.d. & $\mathbf{0 . 1 6}$ \\
\hline
\end{tabular}

\section{A23 $\rightarrow$ Ann}

\begin{tabular}{|c|c|c|c|c|c|}
\hline & GI & GII & GIII & Alle \\
\hline $\mathrm{A} 23 \rightarrow \mathrm{A} 25$ & $1-1$ & 9 & 0 & 0 & 9 \\
\hline & $1-0$ & 10 & 13 & 1 & 24 \\
\hline & $0-1$ & 4 & 2 & 0 & 6 \\
\hline & $0-0$ & 3 & 11 & 17 & 31 \\
\hline Trennschärfe & & -0.09 & -0.29 & n.d. & 0.13 \\
\hline
\end{tabular}

\begin{tabular}{|c|c|c|c|c|c|}
\cline { 3 - 6 } \multicolumn{2}{c|}{} & GI & GII & GIII & Alle \\
\hline A23 $\rightarrow$ A30 & $1-1$ & 10 & 0 & 0 & 10 \\
\hline \multirow{4}{*}{\begin{tabular}{c} 
Trennschärfe \\
\cline { 2 - 6 }
\end{tabular}} & $1-0$ & 9 & 13 & 1 & 23 \\
\cline { 2 - 6 } & $0-1$ & 0 & 1 & 0 & 1 \\
\cline { 2 - 6 } & $0-0$ & 7 & 12 & 17 & 36 \\
\cline { 2 - 6 } & & 0.48 & -0.20 & n.d. & $\mathbf{0 . 3 8}$ \\
\hline
\end{tabular}

\section{$\mathrm{A} 25 \rightarrow \mathrm{Ann}$}

\begin{tabular}{|c|c|c|c|c|c|}
\cline { 3 - 6 } \multicolumn{1}{c|}{} & GI & GII & GIII & Alle \\
\hline A25 $\rightarrow$ A37 & $1-1$ & 12 & 2 & 0 & 14 \\
\hline \multirow{4}{*}{ Trennschärfe } & $1-0$ & 1 & 0 & 0 & 1 \\
\cline { 2 - 6 } & $0-1$ & 11 & 13 & 1 & 25 \\
\cline { 2 - 6 } & $0-0$ & 2 & 11 & 17 & 30 \\
\cline { 2 - 6 } & & 0.12 & 0.25 & n.d. & $\mathbf{0 . 4 0}$ \\
\hline
\end{tabular}

A37 $\rightarrow$ A30

\begin{tabular}{|c|c|c|c|c|c|}
\cline { 2 - 6 } \multicolumn{2}{c|}{} & GI & GII & GIII & Alle \\
\hline A37 $\rightarrow$ A30 & $1-1$ & 8 & 0 & 0 & 8 \\
\hline \multirow{4}{*}{\begin{tabular}{c} 
Trennschärfe \\
\cline { 2 - 6 }
\end{tabular}} & $1-0$ & 15 & 15 & 1 & 31 \\
\cline { 2 - 6 } & $0-1$ & 2 & 1 & 0 & 3 \\
\cline { 2 - 6 } & $0-0$ & 1 & 10 & 17 & 28 \\
\cline { 2 - 6 } & & -0.21 & -0.23 & n.d. & $\mathbf{0 . 1 5}$ \\
\hline
\end{tabular}

\begin{tabular}{|c|c|c|c|c|c|}
\cline { 3 - 6 } \multicolumn{2}{c|}{} & GI & GII & GIII & Alle \\
\hline A25 $\rightarrow$ A30 & $1-1$ & 7 & 0 & 0 & 7 \\
\hline \multirow{4}{*}{\begin{tabular}{c} 
Trennschärfe \\
\cline { 2 - 6 }
\end{tabular}} & $1-0$ & 6 & 2 & 0 & 8 \\
\cline { 2 - 6 } & $0-1$ & 3 & 1 & 0 & 4 \\
\hline & $0-0$ & 10 & 23 & 18 & 51 \\
\hline
\end{tabular}

\begin{tabular}{|c|c|c|c|c|c|}
\cline { 3 - 6 } \multicolumn{2}{c|}{} & GI & GII & GIII & Alle \\
\hline A23 $\rightarrow$ A25 & $1-1$ & 16 & 8 & 0 & 24 \\
\hline \multirow{4}{*}{\begin{tabular}{c} 
Trennschärfe \\
\cline { 2 - 6 }
\end{tabular}} & $1-0$ & 3 & 5 & 1 & 9 \\
\cline { 2 - 6 } & $0-1$ & 7 & 7 & 1 & 15 \\
\cline { 2 - 6 } & $0-0$ & 0 & 6 & 16 & 22 \\
\cline { 2 - 6 } & -0.22 & 0.08 & -0.06 & $\mathbf{0 . 3 2}$ \\
\hline
\end{tabular}

Trennschärfe 
Korrelationskoeffiziententabelle für die Aufgaben

A10, A13, A14, A19, A21, A28, A31, A23, A25, A37, A30

\begin{tabular}{|c|c|c|c|c|c|c|c|c|c|c|}
\cline { 2 - 11 } \multicolumn{1}{c|}{} & 13 & 14 & 19 & 21 & 28 & 31 & 23 & 25 & 37 & 30 \\
\hline 10 & 0.33 & 0.11 & 0.36 & 0.25 & 0.27 & 0.04 & 0.18 & 0.34 & 0.28 & 0.16 \\
\hline 13 & & 0.37 & 0.41 & 0.27 & 0.27 & 0.20 & 0.28 & 0.20 & 0.48 & 0.18 \\
\hline 14 & & & 0.27 & 0.29 & 0.41 & 0.18 & 0.34 & 0.22 & 0.45 & 0.32 \\
\hline 19 & & & & 0.13 & 0.16 & 0.11 & 0.20 & 0.31 & 0.27 & 0.25 \\
\hline 21 & & & & & 0.50 & 0.41 & 0.41 & 0.41 & 0.45 & 0.32 \\
\hline 28 & & & & & & 0.36 & 0.40 & 0.39 & 0.31 & 0.18 \\
\hline 31 & & & & & & & 0.34 & 0.22 & 0.18 & 0.16 \\
\hline 23 & & & & & & & & 0.13 & 0.32 & 0.38 \\
\hline 25 & & & & & & & & & 0.40 & 0.44 \\
\hline 37 & & & & & & & & & & 0.15 \\
\hline
\end{tabular}

